\title{
THE ACTION OF A REAL SEMISIMPLE GROUP ON A COMPLEX FLAG MANIFOLD. I: ORBIT STRUCTURE AND HOLOMORPHIC ARC COMPONENTS ${ }^{1}$
}

\author{
BY JOSEPH A. WOLF \\ Table of Contents
}

1. Introduction............................... 1121

Chapter I. Decomposition of a complex flag manifold into real group orbits. . . 1128

2. Basic facts on the orbit structure ..................... 1129

3. The closed orbit ................................. 1134

4. Open orbits: construction, covering and counting ............ 1140

5. Open orbits: coset space structure and holomorphic functions....... 1145

6. Open orbits: invariant measure........................ 1149

7. Integrable orbits............................. 1154

Chapter II. Decomposition of a real group orbit into complex analytic pieces. 1160

8. Holomorphic arc components........................ 1161

9. Global conditions for the components of an orbit............ 1178

Chapter III. Hermitian symmetric spaces...................... 1195

10. Hermitian symmetric spaces: orbit structure and holomorphic arc components........................... 1197

11. Hermitian symmetric spaces: compact subvarieties and Siegel domain realizations............................... 1208

References................................... 1236

1. Introduction. This paper describes a topic that is of some interest in Lie groups and in differential and algebraic geometry. The topic shows good promise of being the "correct" context for explicit realization of those series of irreducible unitary representations of semisimple Lie groups that come into the Plancherel formula, so it probably is also of interest in harmonic analysis. We start with an example.

Let $X$ be the Riemann sphere, viewed as $C \cup\{\infty\}$ via stereographic projection. Then the group $G$ of all holomorphic automorphisms of $X$ consists of the linear fractional transformations

$$
\pm\left(\begin{array}{ll}
a & c \\
b & d
\end{array}\right): z \rightarrow \frac{a z+b}{c z+d}, \quad \operatorname{det}\left(\begin{array}{ll}
a & c \\
b & d
\end{array}\right)=1
$$

Detailed version of an address delivered at the Riverside meeting of the American Mathematical Society on November 16, 1968, by invitation of the Committee to Select Hour Speakers for Far Western Sectional Meetings; received by the editors May 21, 1969.

1 Research partially supported by N.S.F. Grants GP-2439, GP-5798 and GP-8008, and by an Alfred P. Sloan Research Fellowship. 
that expresses $G$ as a complex matrix group $\operatorname{SL}(2, \mathbf{C}) /\{ \pm I\}$. Let $G_{0}=\operatorname{SL}(2, R) /\{ \pm I\}$, subgroup of elements represented by real matrices, so $G$ is the complexification of $G_{0}$. The complex group $G$ is transitive on $X$ but the real group $G_{0}$ is not. In fact $X$ is the union of two open $G_{0}$-orbits

$G_{0}(\sqrt{1})=\{z \in \mathrm{C}: \operatorname{Im} z>0\}$ and $G_{0}(-\sqrt{-1})=\left\{z \in \in_{\mathrm{C}}^{\mathrm{C}}: \operatorname{Im} z<0\right\}$

and the closed $G_{0}$-orbit

$$
G_{0}(0)=\{z \in \mathrm{C}: \operatorname{Im} z=0\} \cup\{\infty\} .
$$

The Cayley transform

$$
c= \pm \frac{1}{\sqrt{2}}\left(\begin{array}{cc}
1 & \sqrt{-1} \\
\sqrt{-1} & 1
\end{array}\right) \in G
$$

of $X$ relates these orbits, so they are

$$
G_{0}\left(x_{0}\right) \text { and } G_{0}\left(c^{2} x_{0}\right) \text { open, } \quad G_{0}\left(c x_{0}\right) \text { closed, } \quad x_{0}=\sqrt{-1} .
$$

The series of irreducible unitary representations of $G_{0}$ are the "discrete series," the "principal series" and the "complementary series." Discrete series representations are the square integrable ones; they can be realized on the spaces of $L^{2}$-holomorphic sections of $G_{0^{-}}$ homogeneous holomorphic line bundles over the open orbits. Principal series representation can be realized on $L^{2}$ (relative to invariant measure for the rotation subgroup of $G_{0}$ ) sections of certain $G_{0}$-homogeneous complex line bundles over the closed orbit. The complementary series does not contribute to Plancherel measure.

In general we start with a complex manifold $X=G / P$, where $G$ is a complex semisimple Lie group and $P$ is a complex subgroup of the sort called parabolic. The latter means any of the following conditions, which are equivalent:

$X=G / P$ is compact,

$X$ is a compact simply connected kaehler manifold, the complex manifold $X$ is a projective variety, $X$ is a closed $G$-orbit in a projective representation.

Then $X$ is a complex flag manifold. Let $G_{0}$ be a real form of $G$, i.e. a real Lie group whose complexification is $G$. Then $G_{0}$ acts on $X$ as a subgroup of $G$. It turns out that there are only a finite number of 
$G_{0}$-orbits on $X$ and that there is just one closed orbit. The decomposition of $X$ into $G_{0}$-orbits, and especially the structure of the open orbits, is studied in some detail in Chapter I.

We then take an arbitrary orbit $G_{0}(x) \subset X$ and partition it into "maximal complex analytic pieces" that we call its holomorphic arc components. If $z \in G_{0}(x)$ then $S_{[z]}$ denotes the holomorphic arc component of $G_{0}(x)$ through $z$ and $N_{[z], 0}$ denotes the identity component of the Lie subgroup $\left\{g \in G_{0}: g S_{[z]}=S_{[z]}\right\}$; then $S_{[z]}=N_{[z], 0}(z)$ so it is a real submanifold of $X$. The best situation is that in which some (hence every) $S_{[z]} \subset G_{0}(x)$ carries a positive $N_{[z], 0}$-invariant Radon measure; then we say that $G_{0}(x)$ is measurable. If $G_{0}(x)$ is measurable and $z \in G_{0}(x)$ then $S_{[z]}$ is a complex submanifold of $X$, in fact is an open $N_{[z], 0}$-orbit on the complex flag submanifold $N_{[z], 0}^{C}(z)$ of $X$, and its invariant measure is the volume element of an $N_{[z], 0}$-invariant, possibly indefinite signature, kaehler metric. For example, the closed $G_{0}$-orbit on $X$ is always measurable, and we have a method for deciding measurability of the open $G_{0}$-orbits. Holomorphic arc components and global conditions on them such as measurability of the orbit, are studied in Chapter II.

In Chapter III we work out the hermitian symmetric case in complete detail, extending earlier work of Korányi-Wolf [2], WolfKorányi [15] and Takeuchi [9]. This works well because every orbit is measurable. The Riemann sphere, described above, is the simplest of the hermitian symmetric cases; in fact its extreme simplicity can be misleading, so we look at the example of a general complex Grassmann manifold.

Let $X$ be the complex Grassmann manifold consisting of the $k$ planes through the origin in $\mathrm{C}^{n}$. If $\left\{v_{1}, \cdots, v_{k}\right\} \subset \mathrm{C}^{n}$ is linearly independent then $v_{1} \wedge \cdots \wedge v_{k} \in X$ denotes its span. The complex general linear group acts on $X$ by $g: v_{1} \wedge \cdots \wedge v_{k} \rightarrow g\left(v_{1}\right) \wedge \cdots \wedge g\left(v_{k}\right)$; there the scalars act trivially so the resulting group of transformations is the complex Lie group

$$
G=\mathbf{G L}(n, \mathbf{C}) /\{a I: a \neq 0\}=\mathbf{S L}(n, \mathbf{C}) /\left\{e^{2 \pi i r / n} I\right\}
$$

of complex dimension $n^{2}-1$. Let $\left\{e_{1}, \cdots, e_{n}\right\}$ be a fixed basis of $\mathbf{C}^{n}$ and $x_{0}=e_{1} \wedge \cdots \wedge e_{k} \in X$ our base point. Then the isotropy subgroup of $G$ at $x_{0}$ is the complex subgroup

$$
P=\left\{g \in \mathrm{SL}(n, \mathrm{C}): g x_{0}=x_{0}\right\} /\left\{e^{2 \pi i r / n} I\right\}
$$


of complex dimension $k^{2}+(n-k)^{2}+n k-1$. That exhibits $X$ as a complex flag manifold ${ }^{2} G / P$ of complex dimension $k(n-k)$.

Consider the hermitian form $\left\langle\sum_{1}^{n} z^{i} e_{i}, \quad \sum_{1}^{n} w^{j} e_{j}\right\rangle=-\sum_{1}^{k} z^{i} \bar{w}^{i}$ $+\sum_{k+1}^{n} z^{i} \bar{w}^{i}$ of signature $(k, n-k)$ on $\mathbf{C}^{n}$. The subgroup of $\mathrm{GL}(n, \mathrm{C})$ preserving it is the indefinite unitary group $\mathrm{U}^{k}(n)$. The latter acts on $X$ as the real group

$$
G_{0}=\mathrm{U}^{k}(n) /\left\{e^{2 \pi i \theta} I: \theta \text { real }\right\}=\mathrm{SU}^{k}(n) /\left\{e^{2 \pi i r / n} I\right\}
$$

whose complexification is $G$. If $x \in X$ we associate a triple $(a, b, c)$ where $\langle$,$\rangle has rank a+b$ on $x$, with $a$ negative squares and $b$ positive ones, and $a+b+c=k$. The only restrictions are

$a+b+c=k, \quad 0 \leqq a \leqq k, \quad 0 \leqq b \leqq n-k, 0 \leqq c \leqq \min (k, n-k)$.

If $y \in G_{0}(x)$ it gives the same triple; if $y \in X$ gives the same triple then Witt's Theorem says $y \in G_{0}(x)$. Thus there are precisely $\frac{1}{2}(l+1)(l+2)$ $G_{0}$-orbits on $X, l=\min (k, n-k)$, given by

$$
G_{0}\left(c_{1} c_{2} \cdots c_{s} c_{s+1}^{2} c_{s+2}^{2} \cdots c_{t}^{2} x_{0}\right), \quad 0 \leqq s \leqq t \leqq l=\min (k, n-k),
$$

where $c_{i}$ are the partial Cayley transforms defined by

$$
\begin{aligned}
& c_{i}\left(e_{i}\right)=\frac{1}{\sqrt{2}}\left(e_{i}+\sqrt{-1} e_{k+i}\right), \quad c_{i}\left(e_{k+i}\right)=\frac{1}{\sqrt{2}}\left(\sqrt{-1} e_{i}+e_{k+i}\right), \\
& c_{i}\left(e_{j}\right)=e_{j} \quad \text { if } i \neq j \neq k+i .
\end{aligned}
$$

For $\left(\prod_{1 \leq i \leq s} c_{i}\right)\left(\prod_{\bullet<j \leq t} c_{j}^{2}\right) x_{0}$ has "signature" $(k-t, t-s, s)$. A glance at determinants shows that there are

$$
\begin{gathered}
l+1 \text { open orbits } G_{0}\left(c_{1}^{2} c_{2}^{2} \cdots c_{t}^{2} x_{0}\right), \quad 0 \leqq t \leqq l, \\
\text { just one closed orbit } G_{0}\left(c_{1} c_{2} \cdots c_{l} x_{0}\right) .
\end{gathered}
$$

More precisely, the closure of $G_{0}\left(c_{1} c_{2} \cdots c_{s} c_{s+1}^{2} c_{s+2}^{2} \cdots c_{l}^{2} x_{0}\right)$ is the union of all $G_{0}\left(c_{1} c_{2} \cdots c_{u} c_{u+1}^{2} c_{u+2}^{2} \cdots c_{v}^{2} x_{0}\right)$ such that $v-u \leqq t-s$ and $t \leqq v$. Thus, for example, in the case $1=k<n$ of complex projective $(n-1)$-space (of which the Riemann sphere is the case $n=2)$ there are

2 Alternatively one can embed $X$ in complex projective space of dimension $\left(\begin{array}{l}n \\ k\end{array}\right)-1$ by Plücker coordinates as follows. If $x \in \mathrm{X}$ has basis $\left\{v_{1}, \cdots, v_{k}\right\}$, say $v_{i}=\sum_{j} a_{k}^{i} e_{j}$, then $x$ goes to the point with homogeneous coordinates given by the determinants of the $\left(\begin{array}{l}c \\ k\end{array}\right)$ matrices $\left(a^{i_{1}}, a^{i_{2}}, \cdots, a^{i_{k}}\right), 1 \leqq j_{1}<\cdots<j_{k} \leqq n$, where $a^{i}={ }^{t}\left(a_{1}^{i}, \cdots, a_{k}^{i}\right)$. The same embedding is achieved as the closed orbit of the projective representation of $\operatorname{SL}(n, \mathrm{C})$ associated to the $k$ th alternation $\Lambda^{k}(\alpha)$ of the usual (vector) representation $\alpha$ of degree $n$. 


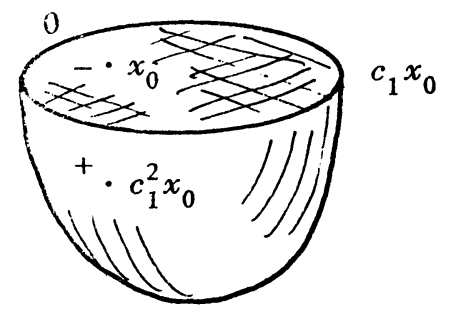

$k=1, n \geqq 2$

- : open: negative definite lines in $\mathbf{C}^{n}$

+ : open: positive definite lines in $\mathbf{C}^{n}$

0: closed: isotropic lines in $\mathbf{C}^{n}$

3 orbits, and in the case $2=k \leqq n / 2$ there are 6 orbits, whose boundary structure is illustrated in the figures. ${ }^{3}$

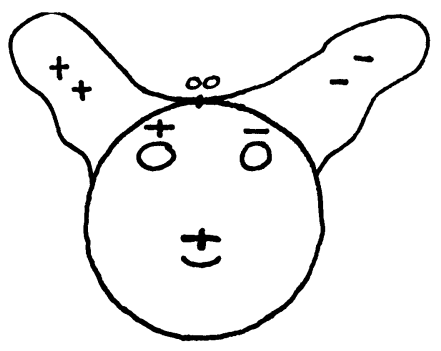

$k=2, n \geqq 4$

- : $G_{0}\left(x_{0}\right)$ open: negative definite planes

$+-: G_{0}\left(c_{1}^{2} x_{0}\right)$ open: indefinite nondegenerate planes

$++: G_{0}\left(c_{1}^{2} c_{2}^{2} x_{0}\right)$ open: positive definite planes

+0: $G_{0}\left(c_{1} c_{2}^{2} x_{0}\right)$ intermediate: degenerate positive semidefinite planes

-0: $G_{0}\left(c_{1} x_{0}\right)$ intermediate: degenerate negative semidefinite planes

00: $G_{0}\left(c_{1} c_{2} x_{0}\right)$ closed: totally isotropic planes

Back with the general complex grassmannian, we note that the open orbit $G_{0}\left(c_{1}^{2} c_{2}^{2} \cdots c_{t}^{2} x_{0}\right)$ consisting of the $k$-planes of signature $(k-t, t)$ has coset space representation $\left.\mathrm{U}^{k}(n) / \mathrm{U}^{t}(k) \times \mathrm{U}^{k-t}(n-k)\right)$; so it has a $G_{0}$-invariant indefinite-kaehler metric of signature $(t(n-2 k), k(n-k)-t(n-2 k))$ whose volume element will prove useful. Indefiniteness of the metric is related to the existence of compact subvarieties. $G_{0}$ has maximal compact subgroup

$$
K=\{\mathrm{U}(k) \times \mathrm{U}(n-k)\} /\left\{\boldsymbol{e}^{2 \pi i \theta} I: \theta \text { real }\right\}
$$

from the maximal compact subgroup $\mathrm{U}(k) \times \mathrm{U}(n-k) \subset \mathrm{U}^{k}(n)$. Now

$$
\begin{aligned}
K\left(c_{1}^{2} c_{2}^{2} \cdots c_{t}^{2} x_{0}\right) & =\{\mathrm{U}(k) / \mathrm{U}(t) \times \mathbf{U}(k-t)\} \times\{\mathrm{U}(n-k) / \mathrm{U}(k-t) \\
& \times \mathbf{U}(n-2 k+t)\}
\end{aligned}
$$

which is the direct product of the complex grassmannians of $t$-planes in $\mathbf{C}^{k}$ and $(k-t)$-planes in $\mathbf{C}^{n-k}$. Moreover the maximal compact sub-

3 The figure for $k=2$ is due to A. Koranyi. 
varieties of the open orbits are the

$$
g \cdot K\left(\underset{c_{1} c_{2}^{2}}{2} \cdots c_{t}^{2} x_{0}\right) \subset G_{0}\left(c_{1}^{2} c_{2}^{2} \cdots c_{t}^{2} x_{0}\right), \quad g \in G_{0}
$$

Furthermore there exist holomorphic fibrations

$$
\beta_{t}: G_{0}\left(c_{1}^{2} c_{2}^{2} \cdots c_{t}^{2} x_{0}\right) \rightarrow K\left(\underset{c_{1} c_{2}^{2}}{2} \cdots c_{t}^{2} x_{0}\right)
$$

given as follows. First notice that the intended base space of $\beta_{t}$ consists of all $v_{1} \wedge \cdots \wedge v_{k-t} \wedge w_{1} \wedge \cdots \wedge w_{t}$ where $v_{1} \wedge \cdots \wedge v_{k-t}$ is a subspace of $e_{1} \wedge \cdots \wedge e_{k}$ and $w_{1} \wedge \cdots \wedge w_{t}$ is a subspace of $e_{k+1} \wedge \cdots \wedge e_{n}$. If $x \in X$ has signature $(k-t, t)$, its orthogonal projection to $e_{1} \wedge \cdots \wedge e_{k}$ is a subspace $v_{1} \wedge \cdots \wedge v_{k-t}$, and its orthogonal projection to $e_{k+1} \wedge \cdots \wedge e_{n}$ is some $w_{1} \wedge \cdots \wedge w_{t}$; by definition $\beta_{t}(x)=v_{1} \wedge \cdots \wedge v_{k-t} \wedge w_{1} \wedge \cdots \wedge w_{t}$. It is not too hard to check that this defines a holomorphic fibre bundle with total space and base as mentioned, whose fibre over $k\left(c_{1}^{2} c_{2}^{2} \cdots c_{t}^{2} x_{0}\right), k \in K$, is naturally isomorphic to the product of open orbits in smaller grassmannians,

negative definite $t$-planes in a $\mathbf{C}^{2 t}$ of signature $(t, t)$ and

negative definite $(k-t)$-planes in a $\mathbf{C}^{n-2 t}$ of signature $(k-t, n-k-t)$.

One can use the maximal compact subvarieties to check directly that the complex manifold $G_{0}\left(c_{1}^{2} c_{2}^{2} \cdots c_{t}^{2} x_{0}\right)$ has nonconstant holomorphic functions if, and only if, either $t=0$ or $t=k=n / 2$. Thus the closest "approximation" to holomorphic functions on $G_{0}\left(c_{1}^{2} c_{2}^{2} \cdots c_{t}^{2} x_{0}\right)$ is that of holomorphic sections of holomorphic line bundles $\beta_{t}^{*} \mathcal{L}$ where $\mathfrak{L} \rightarrow K\left(c_{1}^{2} c_{2}^{2} \cdots c_{t}^{2} x_{0}\right)$ is a holomorphic line bundle with nonzero positive semidefinite Chern class. Look back to the case $k=1$ of complex projective $(n-1)$-space. There $G_{0}\left(x_{0}\right)$ is the unit ball in $\mathbf{C}^{n-1}$, which carries holomorphic functions, and $\beta_{0}$ is fibration over the single point $x_{0}$. The other open orbit $G_{0}\left(c_{1}^{2} x_{0}\right)$ is the exterior of that unit ball with a complex projective $(n-2)$-space $K\left(c_{1}^{2} x_{0}\right)$ attached; if $n>2$ it carries no nonconstant holomorphic functions; $\beta_{1}$ projects it to the polar hyperplane $K\left(c_{1}^{2} x_{0}\right)$ of $x_{0}$, and eligible bundles $\beta_{1}^{*} \mathscr{L}$ are the $\beta_{1}^{*} \mathcal{F C}^{a}, q \geqq 1$, where $\mathfrak{H} \rightarrow K\left(c_{1}^{2} x_{0}\right)$ is the hyperplane bundle over that projective space.

We now cut an arbitrary $G_{0}$-orbit on $X$ into complex submanifolds of $X$. If $g \in G$ (resp. $g \in G_{0}$ ) then $\tilde{g} \in \mathrm{SL}\left(n\right.$, C) (resp. $\tilde{g} \in \mathrm{SU}^{k}(n)$ ) denotes a representing linear transformation of $\mathbf{C}^{n}$. Now define, for $0 \leqq s \leqq l=\min (k, n-k)$,

$$
\begin{aligned}
& G_{[s]}=\left\{g \in G: \tilde{g} e_{i}=e_{i} \text { and } \quad \tilde{g} e_{k+i}=e_{k+i} \quad \text { for } 1 \leqq i \leqq s\right\}, \\
& G_{[s], 0}=\left\{g \in G_{0}: \tilde{g} e_{i}=e_{i} \text { and } \tilde{g} e_{k+i}=e_{k+i} \quad \text { for } 1 \leqq i \leqq s\right\} \text {. }
\end{aligned}
$$


Then

$$
G_{[s]}\left(c_{1} c_{2} \cdots c_{s} x_{0}\right)=G_{[s]}\left(c_{1} c_{2} \cdots c_{s} c_{s+1}^{2} c_{s+2}^{2} \cdots c_{t}^{2} x_{0}\right)
$$

for $s \leqq t \leqq l$, and that manifold is the sub-grassmannian of $X$ consisting of all $(k-s)$-planes in the $\mathbf{C}^{n-2 s}$ with basis $\left\{e_{s+1}, \cdots, e_{k} ; e_{k+s+1}, \cdots, e_{n}\right\}$. Furthermore

$$
G_{[s], 0}\left(c_{1} c_{2} \cdots c_{s} c_{s+1}^{2} c_{s+2}^{2} \cdots c_{t}^{2} x_{0}\right) \text { is open in } G_{[s]}\left(c_{1} c_{2} \cdots c_{s} x_{0}\right) \text {, }
$$

and thus is a complex submanifold of $X$ with an invariant indefinitekaehler metric of signature

$$
((t-s)(n-2 s-2 k), \quad k(n-2 s-k)-(t-s)(n-2 s-2 k)) .
$$

Now the point is that $G_{0}\left(c_{1} c_{2} \cdots c_{s} c_{s+1}^{2} c_{s+2}^{2} \cdots c_{t}^{2} x_{0}\right)$ is a union of submanifolds $k \cdot G_{[s], 0}\left(c_{1} c_{2} \cdots c_{s} c_{s+1}^{2} c_{s+2}^{2} \cdots c_{t}^{2} x_{0}\right)$ with $k \in K$, that any two such submanifolds are equal or disjoint, and that any holomorphic map of the unit disc into $X$ with image in

$$
G_{0}\left(c_{1} c_{2} \cdots c_{s} c_{s+1}^{2} c_{s+2}^{2} \cdots c_{t}^{2} x_{0}\right)
$$

must have image in one of those submanifolds. Thus every $G_{0}$-orbit is decomposed into a disjoint union of indefinite-kaehler submanifolds of $X$ that are maximal among complex submanifolds of $X$ contained in the orbit. They are the holomorphic arc components of the orbit. It now follows that every $G_{0}$-orbit on $X$ is measurable.

Let $y=c_{1} c_{2} \cdots c_{s} c_{s+1}^{2} c_{s+2}^{2} \cdots c_{t}^{2} x_{0}$. Now every holomorphic arc component of the arbitrary $G_{0}$-orbit $G_{0}(y) \subset X$ is an open orbit $k G_{[s], 0} k^{-1}(k y), k \in K$, on the smaller grassmannian $k G_{[s]}(y)$. Thus it has a holomorphic fibration

$$
\beta_{(k)}: k G_{[s], 0}(y) \rightarrow k\left(K \cap G_{[s], 0}\right)(y)
$$

over its maximal compact subvariety. It turns out that the $\beta_{(k)}$ fit together in the sense that

$$
\text { if } k_{1} G_{[\varepsilon], 0}(y)=k_{2} G_{[\varepsilon], 0}(y) \text { then } \beta_{\left(k_{1}\right)}=\beta_{\left(k_{2}\right)} .
$$

The result is a $K$-equivariant fibration

$$
\beta: G_{0}(y) \rightarrow K(y)
$$

whose fibres coincide with those of its restrictions $\beta_{(k)}$, and which thus projects every holomorphic arc component holomorphically to its maximal compact subvariety. Thus one obtains "generalized Siegel domain" realizations of $G_{0}(y)$ by fitting together the standard Siegel domain realizations of the $\beta$-fibres. 
Finally, let me describe the expected relation to representation theory. Let $G_{0}(x) \subset X$ be a measurable orbit. Consider $G_{0}$-homogeneous complex vector bundles $\& \rightarrow G_{0}(x)$ that are holomorphic over every holomorphic arc component of $G_{0}(x)$. Given $\varepsilon$ we form the cohomology groups $H^{p}(\mathcal{E})$ consisting of the $\mathcal{E}$-valued $p$-forms $\omega$ on $G_{0}(x)$ such that $\omega$ is harmonic and of type $(0, p)$ on every holomorphic arc component and

$$
\int_{K} \int_{k \cdot S[x]}\|\omega\|^{2} d \mu_{k} d k<\infty .
$$

Here $d \mu_{k}$ is the (normalized suitably) invariant Radon measure on the arbitrary holomorphic arc component $k \cdot S_{[x]}$, and $d k$ is Haar measure on a maximal compact subgroup $K$ of $G_{0}$. Now the point is to choose $\mathcal{E}$ so that the action of $G_{0}$ on $H^{p}(\mathcal{E})$ is unitary. The evidence is good that measurable open orbits give the discrete series of $G_{0}$, and the (necessarily measurable) closed orbit gives the principal series. The process can be viewed as a specialization of Kostant's theory of symplectic manifolds and polarizations ([4]; see [5] for details) that is set up for semisimple groups. I will go into it in fair detail in [12].

The first third (Chapter I) of this paper represents part of some joint work I did with Bertram Kostant, and that work influenced the second third (Chapter II). Also, a result of Masaru Takeuchi [9] is included in Chapter III for expository reasons. Thus only about $4 / 5$ of this paper is my own work. For that reason there are chapter notes distributing credit in detail at the ends of the introductions to each of the three chapters.

\section{Chapter I. Decomposition of a Complex Flag manifold INTO REAL GROUP ORBITS}

We establish the basic notions for $G_{0}$-orbits on a complex flag manifold $X=G / P, G_{0}$ a real form of $G$, in $\$ 2$; there the root decompositions are set up, the number of $G_{0}$-orbits is bounded, open orbits are shown to exist, and dimensions and codimensions of orbits are computed in such a manner that open orbits are easy to manage. In $\$ 3$ it is shown that there is a unique closed $G_{0}$-orbit on $X$, and various properties are explored for that closed orbit. $\$ 4$ is the initial assault on open orbits; the structure of open orbits is worked out in detail sufficient to supply an enumeration procedure. Open orbits are studied as homogeneous spaces in \$5, where their maximal compact subvarieties are described, where they are proved to be simply connected, and where their holomorphic functions are explicitly described. In \$6 we study the existence problem for invariant Radon measures on open orbits and 
find a complete solution. Finally, in $\$ 7$, the notion of integrable orbit (which is important for Chapter II and application to group representations) is worked out in detail sufficient for an enumeration.

NOTES FOR CHAPTER I. The results of $\$ 2, \S 3$ and $\$ 4$ were proved in some conversations between Bertram Kostant and this author. Theorem 2.6 is essentially the work of Kostant while Theorem 2.12 is due to this author. The material surrounding Theorem 3.3 is primarily the work of Kostant; Theorem 3.6 and its corollaries are essentially due to this author. Theorem 4.5 is due more to Kostant than to this author, and Theorem 4.9 slightly more to this author than to Kostant.

The results of $\$ 5$ were worked out by this author. Lemma 5.1 and Theorem 5.4 are easy in the case where $G_{0} \cap P_{x}$ is compact and our proof may be viewed as a reduction to that case. Theorem 5.7 had been suspected by P. A. Griffiths in the case where $G_{0} \cap P_{x}$ is compact and $G$ is simple; he felt that one should be able to join points of $G_{0}(x)$ by a chain of compact subvarieties under appropriate circumstances; our proof uses a Lie algebra maximality criterion instead.

Theorem 6.3 and its corollaries were worked out jointly in conversations between the author and Bertram Kostant. Then Kostant suggested an examination of the case where $G_{0}$ is complex; that case is described at the end of $\$ 6$; it led to this author working out Theorem 6.7 and its corollary.

The notion of integrable orbit was suggested by Bertram Kostant's work on symplectic homogeneous spaces. Equivalence of (i), (ii) and (iii) in Theorem 7.2 was worked out jointly in conversations between the author and Kostant, and perhaps is due mostly to Kostant. The rest of $\$ 7$ is due to this author.

The conversations between Bertram Kostant and the author that resulted in material in Chapter I took place between August 1965 and February 1967.

2. Basic facts on the orbit structure. Let $G$ be a complex semisimple connected Lie group. $G$ is its Lie algebra. Choose a Cartan subalgebra $\mathfrak{H C} \subset \mathcal{G}$ and a system $\Pi$ of simple roots. If $\varphi$ is a root we denote its coefficients by

$$
\varphi=\sum_{\pi \in \Pi} n \varphi(\pi) \cdot \pi
$$

Suppose $\Phi \subset$ CII. Then the root system $\Delta$ is a disjoint union,

$$
\Delta=\Phi^{r} \cup \Phi^{u} \cup-\Phi^{u}=\Phi^{*} \cup-\Phi^{u}
$$

where we define $\Phi^{*}=\Phi^{r} \cup \Phi^{u}$ and 


$$
\begin{array}{ll}
\Phi^{r}=\left\{\varphi \in \Delta: n_{\varphi}(\pi)=0\right. & \text { for all } \pi \in \Pi-\Phi\}, \\
\Phi^{u}=\left\{\varphi \in \Delta: n_{\varphi}(\pi)>0\right. & \text { for some } \pi \in \Pi-\Phi\} .
\end{array}
$$

That defines subalgebras

$$
\mathcal{P}_{\Phi}^{r}=\mathfrak{H}+\sum_{\Phi^{r}} \oint_{\varphi}, \quad \boldsymbol{P}_{\Phi}^{u}=\sum_{\Phi^{u}} \oint_{\varphi}, \quad \boldsymbol{P}_{\Phi}=\boldsymbol{P}_{\Phi}^{r}+\boldsymbol{P}_{\Phi}^{u}
$$

of $\mathcal{G} . \mathcal{P}_{\Phi}^{r}$ is reductive, $\boldsymbol{P}_{\Phi}^{u}$ is unipotent, $\boldsymbol{P}_{\Phi}$ is their semidirect sum; $\boldsymbol{P}_{\Phi}$ is the normalizer of $\mathcal{P}_{\Phi}^{u}$ in $\mathcal{G} . \mathcal{H}_{\Phi}=\{h \in \mathcal{H}: \Phi(h)=0\}$ is the center of $P_{\Phi}^{r}$.

2.3. Definition. $P_{\Phi}$ is the parabolic subalgebra of $G$ defined by $\preccurlyeq$, II and $\Phi$. If $\Phi$ is empty, so $\mathcal{P}_{\Phi}=\mathfrak{H C}+\sum_{\varphi>0} \oint_{\varphi}$, then $\mathcal{P}_{\Phi}$ is the Borel subalgebra of $\mathcal{G}$ defined by $\mathfrak{H}$ and $\mathrm{II}$.

It is standard that

(i) the Borel subalgebras of $\mathcal{G}$ are just the maximal solvable subalgebras,

(ii) the parabolic subalgebras of $\mathcal{G}$ are just the subalgebras which contain Borel subalgebras of $\mathcal{G}$, and

(iii) given $\mathfrak{K}, \Pi$ and a conjugacy class (inner automorphisms) $\{\mathcal{P}\}$ of parabolic subalgebras of $\mathcal{G}$, there is a unique subset $\Phi \subset \Pi$ such that $\mathcal{P}_{\Phi} \in\{P\}$.

2.4. Definition. $A$ Borel subgroup of $G$ is a maximal complex solvable Lie subgroup. A parabolic subgroup of $G$ is a complex Lie subgroup which contains a Borel subgroup.

It is standard that the following conditions are equivalent for a complex Lie subgroup $L \subset G$ :

(i) $L$ is a parabolic subgroup,

(ii) the Lie algebra of $L$ is a parabolic subalgebra of $G$,

(iii) $L$ is the analytic subgroup of $G$ for a parabolic subalgebra of $G$, and

(iv) $G / L$ is compact.

The Borel subgroups are the parabolic subgroups whose Lie algebras are Borel subalgebras of $\mathrm{G}$. A parabolic subgroup is connected and equal to its own normalizer in $G$.

Let $P \subset G$ be a parabolic subgroup with Lie algebra $P$. Then $g$ has a Cartan subalgebra $\mathfrak{K} \subset \mathcal{P}$, and there is a system $\Pi$ of simple roots and a subset $\Phi \subset \Pi$, such that $P=P_{\Phi}$. Let $P^{r}$ and $P^{u}$ denote the respective analytic subgroups of $P$ for $\rho^{r}=\rho_{\Phi}^{r}$ and $\rho^{u}=\rho_{\Phi}^{u}$. Then $P^{r} \cap P^{u}$ $=\{1\}$, so $P=P^{r} \cdot P^{u}$ semidirect product, and $P$ is the normalizer of $P^{u}$ in $G$.

2.5. Definition. $A$ complex flag manifold is a coset space $X=G / P$, where $G$ is a complex semisimple connected Lie group and $P$ is a parabolic subgroup; $X$ is viewed as a complex manifold and $G$ is viewed as a group of holomorphic transformations of $X$. 
Let $X=G / P$ be a complex flag manifold. If $x \in X$, say $x=g P$, then $\operatorname{ad}(g) P=g P g^{-1}$ is the isotropy subgroup of $G$ at $x$. As $P$ is its own normalizer in $G$ we may identify $x$ with $\operatorname{ad}(g) P$, viewing $X$ as the space of parabolic subgroups of $G$ conjugate to $P$. Whether we do that or not, we often write $P_{x}$ for the parabolic ad $(g) P, x=g P$, corresponding to $x$.

Let $G_{0}$ be a real form of $G$. In other words, $G_{0}$ is the analytic subgroup for a real form $\mathcal{G}_{0}$ of $\mathcal{G}_{\text {. }} \tau$ denotes complex conjugation of $\mathcal{G}$ over So, of $G$ over $G_{0}$; so $G_{0}$ is the identity component of the fixed point set of $\tau$ on $G$.

2.6. Theorem. Let $X=G / P$ be a complex flag manifold. Let $G_{0}$ be a real form of $G$ and let $\tau$ be conjugation of $G$ over $G_{0}$.

1. If $x \in X$ then the isotropy subalgebra $g_{0} \cap \rho_{x}$ is a real form of $\odot_{x} \cap \tau \odot_{x}$ and contains a Cartan subalgebra of $\mathcal{G}_{0}$. Conversely if $\mathfrak{H}_{0}$ is a Cartan subalgebra of $\mathcal{G}_{0}$ then $\mathfrak{H}_{0} \subset \mathcal{G}_{0} \cap \mathcal{P}_{x}$ for some $x \in X$.

2. Let $m$ be the number of conjugacy classes of Cartan subalgebras of Go. Let $W_{G}$ denote the Weyl group of $G$. Then $G_{0}$ has $\leqq m\left|W_{G}\right|$ orbits on $X$.

3. $G_{0}$ has an open orbit on $X$, and the union of the open $G_{0}$-orbits is dense in $X$.

PROOF. $\mathcal{G}_{0} \cap \rho_{x}$ is the isotropy subalgebra of $\mathcal{G}_{0}$ at $x$, and $\mathcal{G}_{0} \cap \rho_{x}$ $=\left(\mathcal{G}_{0} \cap \mathcal{P}_{x}\right) \cap \tau\left(\mathcal{G}_{0} \cap \mathcal{P}_{x}\right)=\mathcal{G}_{0} \cap \mathcal{P}_{x} \cap \tau \mathcal{P}_{x}$; so $\mathcal{G}_{0} \cap \mathcal{P}_{x}$ is a real form of

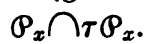

Choose a Borel subalgebra $B \subset \mathcal{P}_{x}$ of $\mathcal{G}$. Then $B \cap \tau \leftrightarrow$ is $\tau$-stable and contains a Cartan subalgebra of $\mathcal{G}$, so it contains a $\tau$-stable Cartan subalgebra $\mathfrak{H}$ of $\mathcal{G}$. Now $\mathfrak{K C} \cap \mathrm{G}_{0}$ is a Cartan subalgebra of $\mathcal{G}_{0}$ contained in $g_{0} \cap P_{x}$.

Let $\mathfrak{F}_{0}$ be a Cartan subalgebra of $\mathcal{S}_{0}$ and let $\mathfrak{F}=\mathfrak{H}_{0}^{\mathfrak{e}}$, Cartan subalgebra of $\mathcal{G}$. Choose a system $\Pi$ of simple roots and a subset $\Phi \subset \Pi$ such that $\mathcal{P}_{\Phi}$ is conjugate to $\mathcal{P}$ in $\mathcal{G}$. Then $\mathcal{P}_{\Phi}=\mathcal{P}_{x}$ for some $x \in X$, and $\mathcal{F}_{0} \subset \mathcal{G}_{0} \cap \mathcal{P}_{x}$.

This completes the proof of (1).

Let $\left\{\mathfrak{K}_{1}, \cdots, \mathfrak{K}_{m}\right\}$ represent the conjugacy classes of Cartan subalgebras of $\mathcal{G}_{0}$. Given $\mathfrak{K}_{i}$ we have $\left|W_{G}\right|$ systems II of simple $\mathcal{F}_{i}^{e}$-roots of $\mathcal{G}$. Given $\Pi$ we have a unique subset $\Phi \subset \Pi$ such that $\mathcal{P}_{\Phi}=\mathcal{P}_{x}$ for some $x \in X$, and that specifies an orbit $G_{0}(x)$. We have just described $m \cdot\left|W_{G}\right|$ orbits of $G_{0}$ on $X$; they need not be distinct, but we must check that every $G_{0}$-orbit is one of them. Let $z \in X$. Then $\mathcal{S}_{0}$ has a Cartan subalgebra $\mathfrak{H C}_{0} \subset \mathcal{G}_{0} \cap \mathcal{P}_{z}$. We have $1 \leqq i \leqq m$ and $g \in G$ such that $\operatorname{ad}(g) \mathfrak{H C}_{0}=\mathfrak{H C}_{i}$; now $\mathfrak{H C}_{i} \subset \mathcal{G}_{0} \cap \odot_{g(z)}$ so we have a $\Pi$ and a subset $\Phi \subset \Pi$ such that $\mathcal{P}_{g(z)}=\mathcal{P}_{\Phi}$; thus $G_{0}(z)=G_{0}(x)$ where $\mathcal{P}_{\Phi}=\mathcal{P}_{x}$. This proves (2). 
Now $G_{0}$ has only a finite number of orbits on $X$. Thus some $G_{0}$-orbit has full dimension; hence is open, and the union of the lower dimensional $G_{0}$-orbits is a closed lower dimensional subset of $X$. This proves (3). Q.E.D.

Now the case of compact $G_{0}$, which is not new, becomes rather easy:

2.7. Corollary. Let $X=G / P$ be a complex flag manifold. Let $G_{u}$ be a compact real form of $G$. Then $G_{u}$ is transitive on $X$, so $X=G_{u} /\left(G_{u} \cap P\right)$, and the isotropy subgroup $G_{u} \cap P$ is the centralizer of a torus. In particular $X$ is simply connected, the integral cohomology $H^{*}(X ; Z)$ is torsion free, and $H^{q}(X ; \mathbf{Z})=0$ for $q$ odd.

Proof. Let $G_{u}(x)$ be an open orbit. It is closed because $G_{u}$ is compact. Thus $G_{u}(x)=X$.

Let $\mathcal{F C}_{u} \subset \mathcal{S}_{u} \cap \beta$ be a Cartan subalgebra of $\mathcal{S}_{u}, \mathfrak{K}=\mathfrak{H C}_{u}^{C}$, II a simple root system, and $\Phi \subset \Pi$, such that $\rho=\mathcal{P}_{\Phi}$. $i \mathcal{C}_{u}$ contains the roots so

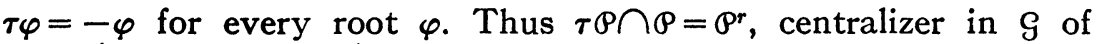
$\mathfrak{H C}_{\Phi}=\{h \in \mathcal{H C}: \Phi(h)=0\}$; now $\mathcal{P}_{u}^{r}=\mathcal{G}_{u} \cap \beta$ is a real form of $\rho^{r}$, centralizer in $S_{u}$ of $S=\left\{h \in \mathcal{F}_{u}: \Phi(h)=0\right\}$. Let $S$ be the toral subgroup of $G_{u}$ with Lie algebra $S$. Now the centralizer of $S$ in $G_{u}$ is connected and contains $G_{u} \cap P$, and is the identity component of $G_{u} \cap P$. Thus $G_{u} \cap P$ is the centralizer of $S$; in particular $X$ is simply connected and it follows from results of Bott-Samelson [1] that $H^{*}(X, Z)$ is torsion free, nonzero only in even degrees. Q.E.D.

2.8. Corollary. Let $P_{1} \subset P_{2}$ be parabolic subgroups of $G$ so $X_{i}=G / P_{i}$ are complex flag manifolds. Let $\pi: X_{1} \rightarrow X_{2}$ be the natural $G$-equivariant fibration. Then the fibre of $\pi$ is a complex flag manifold $Y=\left(P_{2}^{r}\right)^{\prime} / P_{1} \cap\left(P_{2}^{r}\right)^{\prime}$, and, relative to any coefficient ring, Poincaré polynomials satisfy

$$
\mathbf{p}\left(X_{1}, t\right)=\mathbf{p}\left(X_{2}, t\right) \cdot \mathbf{p}(Y, t) .
$$

Proof. $\pi$ has fibre $F=P_{2} / P_{1}$. Choose a Cartan subalgebra $\mathfrak{H C} \subset P_{1}$ of $\mathcal{G}$, a system $\Pi$ of simple roots, and subsets $\Phi_{1} \subset \Phi_{2}$ of $\Pi$, such that $\boldsymbol{P}_{i}=\boldsymbol{P}_{\Phi_{i}}$. Then $\boldsymbol{P}_{1}^{r} \subset \mathcal{P}_{2}^{r}$ and $\boldsymbol{P}_{1}^{u} \supset \mathcal{P}_{2}^{u}$, so $P_{2}^{u}$ acts trivially and $P_{2}^{r}$ acts transitively on $F$. As $P_{1}$ contains the center of $P_{2}^{r}$, the semisimple part $\left(P_{2}^{r}\right)^{\prime}$ acts transitively on $F$, and its isotropy subgroup $P_{1} \cap\left(P_{2}^{r}\right)^{\prime}$ is a parabolic subgroup. This realizes $F$ as the complex flag manifold $Y$. All three of $X_{1}, X_{2}$ and $Y$ now have no integral cohomology in odd degrees and no torsion in even degrees, so the spectral sequence of $\pi$ is trivial. Q.E.D.

We take a closer look at the $G_{0}$-orbits on $X=G / P$. Let $x \in X$, choose a Cartan subalgebra $\mathfrak{H}_{0} \subset \mathcal{G}_{0} \cap \mathcal{P}_{x}$ of $\mathcal{G}_{0}$, let $\mathfrak{H C}=\mathfrak{H C}_{0}^{C}$, and choose 
a simple root system $\Pi$ and a subset $\Phi$ such that $\boldsymbol{P}_{x}=\boldsymbol{P}_{\Phi}$. Now we define

$$
\begin{aligned}
l & =\operatorname{rank} \mathcal{G}, \text { i.e. } l=\operatorname{dim}_{C} \mathfrak{H}=\operatorname{dim}_{R} \mathfrak{H}_{0}, \\
r & =\text { number of positive roots of } \mathcal{G}, \\
\boldsymbol{r}_{\Phi} & =\text { number of positive roots of } \boldsymbol{P}_{\Phi}^{r} .
\end{aligned}
$$

Then $\operatorname{dim}_{C} G=l+2 r, \operatorname{dim}_{C} \rho^{r}=l+2 r_{\Phi}, \operatorname{dim}_{C} \beta^{u}=r-r_{\Phi}, \operatorname{dim}_{C} \beta=l$ $+r+r_{\Phi}$ and $\operatorname{dim}_{C} X=r-r_{\Phi}$.

2.10. LEMMA. $\odot_{x} \cap \tau \odot_{x}$ is the semidirect sum of the reductive algebra $\left(\odot_{x} \cap \tau \odot_{x}\right)^{r}$ given by

$$
\rho_{\Phi}^{r} \cap \tau P_{\Phi}^{r}=\mathcal{H}+\sum_{\Phi^{r} \cap \tau \Phi^{r}} \mathcal{G}_{\varphi}
$$

with the nilpotent ideal $\left(\odot_{x} \cap \tau \odot_{x}\right)^{u}$ given by

$$
\begin{aligned}
\left(\rho_{\Phi}^{r} \cap \tau \rho_{\Phi}^{u}\right)+\left(\rho_{\Phi}^{u} \cap \tau \rho_{\Phi}^{r}\right) & +\left(\rho_{\Phi}^{u} \cap \tau \odot_{\Phi}^{u}\right) \\
& =\left\{\sum_{\Phi^{r} \cap \tau \Phi^{u}}+\sum_{\Phi^{u} \cap \tau \Phi^{r}}+\sum_{\Phi^{u} \cap \tau \Phi^{u}}\right\}\left(S_{\varphi}\right) .
\end{aligned}
$$

In particular

$$
\operatorname{dim}_{R}\left(\mathcal{S}_{0} \cap \rho_{x}\right)=\operatorname{dim}_{C}\left(\odot_{x} \cap \tau \odot_{x}\right)=\operatorname{dim}_{C}{\odot_{\Phi}^{r}}^{r}\left|\Phi^{u} \cap \tau \Phi^{u}\right| .
$$

PROOF. $\mathcal{P}_{x} \cap \tau \mathcal{P}_{x}$ is the sum of $\mathcal{H}$ and certain $\mathcal{G}_{\varphi}$, with $\mathcal{G}_{\varphi}$ in the reductive part (resp. nilpotent ideal) if and only if $\mathcal{G}_{-\varphi}$ also occurs (resp. does not occur) in $\mathcal{P}_{x} \cap \tau \mathcal{P}_{x}$. Thus $\left(\mathcal{P}_{x} \cap \tau \mathcal{P}_{x}\right)^{r}$ is the sum of $\mathcal{F}$ and $\left\{g_{\varphi}\right.$ : both $\varphi$ and $-\varphi$ are in $\left.\Phi^{*} \cap \Phi^{*}\right\}$, i.e. the sum of $\mathcal{F}$ and $\left\{\mathcal{S}_{\varphi}: \varphi \in \Phi^{r} \cap \tau \Phi^{r}\right\}$, as asserted, and $\left(\odot_{x} \cap \tau \odot_{x}\right)^{u}$ is the sum of all $\mathcal{S}_{\varphi}$ for $\varphi \in \Phi^{*} \cap \tau \Phi^{*}$ but $\varphi \notin \Phi^{r} \cap \tau \Phi^{r}$, i.e. $\varphi \in\left(\Phi^{r} \cap \tau^{u}\right) \cup\left(\Phi^{u} \cap \tau \Phi^{r}\right)$ $\cup\left(\Phi^{u} \cap \tau \Phi^{u}\right)$ as asserted.

Now $\operatorname{dim}_{C}\left(\odot_{x} \cap \tau \odot_{x}\right)=l+\left|\Phi^{r} \cap \tau \Phi^{r}\right|+\left|\Phi^{r} \cap \tau \Phi^{u}\right|+\left|\Phi^{u} \cap \tau \Phi^{r}\right|$ $+\left|\Phi^{u} \cap \tau \Phi^{u}\right|$. But $\operatorname{dim}_{C} \quad \rho_{\Phi}^{r}=l+\left|\Phi^{r}\right|=l+\left|\Phi^{r} \cap \tau \Phi^{r}\right|+\left|\Phi^{r} \cap \tau \Phi^{u}\right|$ $+\left|\Phi^{r} \cap-\tau \Phi^{u}\right|$, and $\Phi^{u} \cap \tau \Phi^{r}=-\tau\left(\Phi^{r} \cap-\tau \Phi^{u}\right)$ implies $\left|\Phi^{r} \cap-\tau \Phi^{u}\right|$ $=\left|\Phi^{u} \cap \tau \Phi^{r}\right|$. Thus $\operatorname{dim}_{C}\left(\odot_{x} \cap \tau \odot_{x}\right)-\operatorname{dim}_{C} P_{\Phi}^{r}=\left|\Phi^{u} \cap \tau \Phi^{u}\right|$. Q.E.D.

2.12. Theorem. Let $X=G / P$ be a complex flag manifold, $x \in X$ and $P_{x}=P_{\Phi}$ as above. Then

(i) $\operatorname{dim}_{C} X=r-r_{\Phi}$,

(ii) $\left|\Phi^{u} \cap \tau \Phi^{u}\right|$ is the real codimension of the orbit $G_{0}(x)$ in $X$, and

(iii) $G_{0}(x)$ is open in $X$ if and only if $\Phi^{u} \cap \tau \Phi^{u}$ is empty. 
Proof. (i) was noted as an immediate consequence of the definitions of $r$ and $r_{\Phi}$.

For (ii) we calculate

$$
\begin{aligned}
\operatorname{codim}_{R} G_{0}(x) & =\operatorname{dim}_{R} X-\operatorname{dim}_{R} G_{0}(x) \\
& =2 \operatorname{dim}_{C} X-\left\{\operatorname{dim}_{R} G_{0}-\operatorname{dim}_{R} G_{0} \cap P_{x}\right\} \\
& =\left(2 r-2 r_{\Phi}\right)-\left\{(l+2 r)-\left(l+2 r_{\Phi}+\left|\Phi^{u} \cap \tau \Phi^{u}\right|\right)\right\} \\
& =\left|\Phi^{u} \cap \tau \Phi^{u}\right| .
\end{aligned}
$$

For (ii) we note that $G_{0}(x)$ is open in $X$ if and only if $\operatorname{codim}_{R} G_{0}(x)$ $=0$. Q.E.D.

Now the orbit $G_{0}(x)$ has real dimension $2\left(r-r_{\Phi}\right)-\left|\Phi^{u} \cap \Phi^{u}\right|$, maximized as $2\left(r-r_{\Phi}\right)$ for the open orbits, minimized for a closed orbit.

3. The closed orbit. Let $x \in X$. Then either $G_{0}(x)$ is closed in $X$ or its topological boundary is a nonempty union of lower dimensional orbits. Thus any $G_{0}$-orbit of minimal dimension is closed.

Let $G_{0}(x)$ be a closed orbit. In other words $G_{0} /\left(G_{0} \cap P_{x}\right)$ is compact. Thus there is a maximal compact subgroup $K \subset G_{0}$ and an Iwasawa decomposition $G_{0}=K A N$, such that $A N$ is contained in the isotropy subgroup $\left(P_{x} \cap \tau P_{x}\right)_{0}=G_{0} \cap P_{x}$ of $G_{0}$ at $x$. Extend $A$ to a Cartan subgroup $H_{0}=T \times A$ of $G_{0}$, where $T \subset K$ is a maximal torus of the centralizer $M$ of $A$ in $K$. Then $M A N$ is a minimal parabolic subgroup of $G_{0}$ and $\left(P_{x} \cap \tau P_{x}\right)_{0}$ contains $T A N$. We have proved

3.1. Lemma. $G_{0}(x)$ is a closed $G_{0}$-orbit in $X$ if and only if there is an Irwasawa decomposition $G_{0}=K A N$, the corresponding minimal parabolic subgroup $M A N$ of $G_{0}$, and a maximal torus $T$ of $M$, such that $T A N \subset G_{0} \cap P_{x}$.

Now suppose that $P$ is a Borel subgroup of $G_{0}$, so $\mathcal{P}_{x} \cap \tau \odot_{x}$ is solvable. Let $\mathfrak{F}=\mathfrak{F C}_{0}^{e}$ and let $\varphi$ be an $\mathfrak{K}$-root. First suppose $\varphi(Q) \neq 0$. If $\mathcal{G}_{\varphi} \subset x^{\boldsymbol{e}}$ then $\mathcal{G}_{-\varphi} \subset x^{\mathbf{e}}$, so $\mathcal{G}_{\varphi} \subset \mathcal{P}_{x} \cap \tau \mathcal{P}_{x}$ because the latter is solvable. Now suppose $\varphi(Q)=0$ so $\mathcal{S}_{\varphi} \subset \mathscr{N}^{\mathrm{e}}$. If $\mathcal{S}_{\varphi} \subset \mathcal{P}_{x} \cap \tau \mathcal{P}_{x}$ then $\mathcal{G}_{-\varphi} \subset \mathcal{P}_{x} \cap \tau \mathcal{P}_{x}$ because $M$ is compact, contradicting solvability of $\rho_{x} \cap \tau \mathcal{P}_{x}$. We have proved

3.2. Lemma. Let $X=G / B$ where $B$ is a Borel subgroup. Let $G_{0}(x)$ be $a$ closed orbit. Then there is an Iwasawa decomposition $G_{0}=K A N$, the corresponding minimal parabolic subgroup $M A N$ of $G_{0}$, and a maximal torus $T$ of $M$, such that $\mathcal{G}_{0} \cap \mathcal{P}_{x}=J+Q+\Re$.

Now we can prove uniqueness. 
3.3. Theorem. Let $X=G / P$ be a complex flag manifold, $G_{0}$ a real form of $G$. Then there is a unique closed $G_{0}$-orbit on $X$.

Proof. Let $B C P$ be a Borel subgroup of $G$ and let $Y=G / B$ be the corresponding complex flag manifold. We have the natural projection $\pi: Y \rightarrow X$ with compact fibres. Let $x \in X$. Then $\pi^{-1} G_{0}(x)$ is a union of $G_{0}$-orbits on $Y$. If $G_{0}(x)$ is closed, hence compact, then $\pi^{-1} G_{0}(x)$ is closed, so $\pi^{-1} G_{0}(x)$ contains a closed $G_{0}$-orbit in $Y$. If $G_{0}\left(x_{1}\right)$ and $G_{0}\left(x_{2}\right)$ are distinct closed $G_{0}$-orbits on $X$, now their respective inverse images contain distinct closed $G_{0}$-orbits on $Y$. Thus we need only prove our uniqueness assertion on $Y$. In other words the proof is reduced to the case where $P$ is a Borel subgroup of $G$.

Now $P$ is a Borel subgroup of $G$. Fix an Iwasawa decomposition $G_{0}=K A N$, the corresponding minimal parabolic subgroup $M A N$ of $G_{0}$, and a maximal torus $T$ of $M$. If $G_{0}(x)$ is closed then we have other choices $G_{0}=K^{\prime} A^{\prime} N^{\prime}, M^{\prime} A^{\prime} N^{\prime}$ and $T^{\prime} \subset M^{\prime}$, such that $g_{0} \cap \rho_{x}=J^{\prime}$ $+a^{\prime}+\mathfrak{r}^{\prime}$. Any two Iwasawa decompositions of $G_{0}$ are conjugate. So we have $g \in G_{0}$ such that ad $(g)$ sends $K^{\prime}$ to $K, A^{\prime}$ to $A$ and $N^{\prime}$ to $N$. Automatically ad $(\mathrm{g}) M^{\prime}=M$. Any two maximal tori of $M$ are conjugate, and $M$ normalizes both $A$ and $N$; thus we may also assume $\operatorname{ad}(g) T^{\prime}=T$. Replacing $x$ by $g(x)$, the orbit $G_{0}(x)=G_{0}(g x)$ is not changed, and we may assume $\mathcal{G}_{0} \cap \odot_{x}=\mathfrak{J}+Q+\Re$. Now the Borel subalgebra $\mathcal{P}_{x}=\mathfrak{L}+\mathfrak{Q}^{\mathfrak{e}}+\mathfrak{x}^{\mathfrak{e}}$ where, for some ordering, $\mathfrak{L}$ is the sum of $\mathfrak{J}^{\mathrm{e}}$ with the positive $\mathfrak{J}^{\mathrm{e}}$-root spaces of $\mathfrak{m}^{\mathrm{e}}$. Conjugating further by an element $g \in M \subset G_{0}$ we may assume that ordering to be a given one.

Let $G_{0}\left(x_{1}\right)$ and $G_{0}\left(x_{2}\right)$ be closed orbits on $X$. We have just seen that $x_{i}$ can be replaced within $G_{0}\left(x_{i}\right)$ so that $\mathcal{P}_{x_{i}}=\mathfrak{L}+a^{\mathfrak{e}}+\mathfrak{r}^{\mathfrak{e}}$ in the notation above, i.e. so that $\mathcal{P}_{x_{1}}=\mathcal{P}_{x_{2}}$, i.e. so that $x_{1}=x_{2}$. Thus $G_{0}\left(x_{1}\right)$ $=G_{0}\left(x_{2}\right)$. Q.E.D.

An immediate consequence is

3.4. Corollary. Let $X=G / P$ be a complex flag manifold, $x \in X$, and $G_{0}$ a real form of $G$. Then the following conditions are equivalent:

(i) $G_{0}(x)$ is the unique closed $G_{0}$-orbit.

(ii) $G_{0}(x)$ is contained in the closure of every $G_{0}$-orbit on $X$.

(iii) $G_{0}(x)$ is contained in every $G_{0}$-stable closed subset of $X$.

(iv) Some maximal compact subgroup of $G_{0}$ is transitive on $G_{0}(x)$.

(v) Every maximal compact subgroup of $G_{0}$ is transitive on $G_{0}(x)$.

(vi) $G_{0}(x)$ is the lowest-dimensional $G_{0}$-orbit on $X$.

Proof. Equivalence of (i), (ii), (iii) and (vi) follows from uniqueness of the closed orbit and the fact that it is on the boundary of every nonclosed orbit. Trivially (v) implies (iv) and (iv) implies (i), so we need only check that (i) implies (v). Choose a Borel subgroup $B C P$ 
of $G$, let $Y=G / B$ and let $\pi: Y \rightarrow X$ be the projection. $G_{0}(x)$ is closed, so $\pi^{-1} G_{0}(x)$ is closed, so $\pi^{-1} G_{0}(x)$ contains a closed orbit $G_{0}(y)$. In the notation of the lemmas, $B_{y} \cap g_{0}=\Im+a+\Re$ where $G_{0}=K A N$. Let $K^{\prime}$ be any maximal compact subgroup of $G_{0}$. Then $K^{\prime} \cap A N=\{1\}$, so

$$
\begin{aligned}
\operatorname{dim} G_{0}(y) & \geqq \operatorname{dim} K^{\prime}(y)=\operatorname{dim} K^{\prime}-\operatorname{dim}\left(K^{\prime} \cap T A N\right) \\
& \geqq \operatorname{dim} K^{\prime}-\operatorname{dim} T=\operatorname{dim} K-\operatorname{dim} T \\
& =\operatorname{dim} K(y)=\operatorname{dim} K / T=\operatorname{dim} G_{0} / T A N \\
& =\operatorname{dim} G_{0}(y),
\end{aligned}
$$

proving $K^{\prime}(y)=G_{0}(y)$. Now $G_{0}(x)=\pi G_{0}(y)=\pi K^{\prime}(y)=K^{\prime}(x)$. Q.E.D.

We just made implicit use of the obvious consequence of uniqueness of the closed orbit:

3.5. Corollary. Let $P_{1} \subset P_{2}$ be parabolic subgroups of $G, X_{i}=G / P_{i}$ the resulting complex flag manifolds, and $\pi: X_{1} \rightarrow X_{2}$ the natural projection. Let $G_{0}$ be a real form of $G, x_{i} \in X_{i}$ such that $G_{0}\left(x_{i}\right)$ is the closed orbit on $X_{i}$. Then

$$
\pi G_{0}\left(x_{1}\right)=G_{0}\left(x_{2}\right) \text { and } G_{0}\left(x_{1}\right) \subset \pi^{-1} G_{0}\left(x_{2}\right) .
$$

Now we count dimensions, proving

3.6. Theorem. Let $X=G / P$ be a complex flag manifold, $x \in X, G_{0} a$ real form of $G$, and $\rho_{x}=\odot_{\Phi}$ relative to a simple root system on a $\tau$-stable Cartan subalgebra $\mathfrak{H C} \subset \odot_{x}$ of $\mathcal{G}$.

1. $\operatorname{dim}_{R} G_{0}(x) \geqq \operatorname{dim}_{\mathrm{e}} X \quad\left\{=\frac{1}{2} \operatorname{dim}_{R} X\right\}$.

2. The following conditions are equivalent:

(2a) $\operatorname{dim}_{R} G_{0}(x)=\operatorname{dim}_{C} X$.

(2b) $\tau \Phi^{u}=\Phi^{u}$.

(2c) $\tau \odot_{x}=P_{x}$, i.e. $\tau P_{x}=P_{x}$, i.e. viewing $G$ as a linear algebraic group defined/ $R$ such that $G_{0}$ is the topological identity component of the subgroup $G_{\mathbf{R}}$ of real points, the algebraic subgroup $P_{x}$ of $G$ is $\operatorname{def} / \boldsymbol{R}$.

(2d) $G_{0}(x)$ is the closed orbit; with $G$ def/R as in (2c), some conjugate of $P$ is def $/ \mathbf{R}$.

(2e) $X$ is a complex projective variety $\mathrm{def} / \mathrm{R}$ in such a manner that $G_{0}(x)$ is the set $X_{\mathbf{R}}$ of real points.

PROOF. $\operatorname{dim}_{R} G_{0}(x)=2\left(r-r_{\Phi}\right)-\left|\Phi^{u} \bigcap \tau \Phi^{u}\right| \geqq 2\left(r-r_{\Phi}\right)-\left|\Phi^{u}\right|=r-r_{\Phi}$ $=\operatorname{dim}_{e} X$, with equality if and only if $\Phi^{u}=\tau \Phi^{u}$. This proves (1) and gives equivalence of (2a) and (2b). $\tau \Phi^{u}=\Phi^{u}$ if and only if $\tau \boldsymbol{P}_{\Phi}^{u}=\boldsymbol{P}_{\Phi}^{u}$ because $\tau \mathcal{H}=\mathcal{H}, \tau \mathcal{P}_{\Phi}^{u}=\mathcal{P}_{\Phi}^{\mu}$ if and only if $\tau \mathcal{P}_{\Phi}=\mathcal{P}_{\Phi}$ because $\mathcal{P}_{\Phi}$ (resp. $\tau \mathcal{P}_{\Phi}$ ) is the normalizer of $\boldsymbol{P}_{\Phi}^{u}$ (resp. $\tau \boldsymbol{P}_{\Phi}^{u}$ ) in $\mathcal{G}$; this gives equivalence of $(2 b)$ and (2c). 
(1), the characterization of the closed orbit as the orbit of lowest dimension, and equivalence of (2a) and (2c), show that (2c) implies (2d). Conversely let $G_{0}(x)$ be closed and some $P_{z}$ def/R; then $\operatorname{dim}_{R} G_{0}(z)=\operatorname{dim}_{\mathrm{e}} X$, so $G_{0}(z)$ is a lowest dimensional orbit, so $G_{0}(x)$ $=G_{0}(z)$ and we have (2a). Thus (2c) and (2d) are equivalent.

(2e) implies (2a), thus (2c). Conversely assume (2c). Then the projective variety $X$ is def $/ \mathrm{R}$ and $G_{0}(x)$ is a topological component of $X_{R}$. But $X_{R}$ is a finite union of topological components $X_{R}^{(i)}, G_{0}$ is transitive on each $X_{R}^{(i)}$, each $X_{R}^{(i)}$ is closed in $X$, and there is just one closed $G_{0}$-orbit. Now there is just one $X_{R}^{(i)}$, so $G_{0}(x)=X_{R}$ and we have (2e). Thus (2c) and (2e) are equivalent. Q.E.D.

We reformulate condition (2c) of Theorem 3.6 in terms of the action of the Galois group of $\mathbf{C}$ over $\mathbf{R}$ on a simple root system.

3.7. Corollary. Let $X=G / P$ be a complex flag manifold, $x \in X$, $G_{0}$ a real form of $G, \mathfrak{H} \subset \mathcal{P}_{x}$ a $\tau$-stable Cartan subalgebra of $\mathcal{G}$, and $\Pi$ II simple root system such that $\mathcal{P}_{x}=\mathcal{P}_{\Phi}$ for some $\Phi \subset$ III. Define $\nu=w \cdot \tau$ where $w$ is an element of the Weyl group which sends $\tau(\mathrm{II})$ back to $\Pi$. Then $\operatorname{dim}_{R} G_{0}(x)=\operatorname{dim}_{\mathfrak{e}}(X)$ if and only if

(i) $\nu \Phi=\Phi$ and

(ii) there is a minimal parabolic subgroup $M A N C G_{0}$ such that $\Re^{\mathfrak{e}}+a^{\mathfrak{e}}+\mathfrak{\kappa}^{\mathfrak{e}}=\boldsymbol{P}_{\Psi}$ with $\Psi \subset \Phi$.

Proof. $\{1, \nu\}$ is the action of the Galois group of $C$ over $R$ on the simple root system $\Pi$, and (ii) is the statement that $\mathcal{P}_{x} \cap \mathcal{G}_{0}$ contains a minimal parabolic subalgebra of $g_{0}$. Now (i) and (ii) just say that $\tau \mathcal{P}_{x}=\mathcal{P}_{x}$. Q.E.D.

We reformulate condition (2e) of Theorem 3.6 in terms of linear representations. To do that we order the weights by $\Pi$ and say that a positive weight $\lambda$ belongs to the subset $\Phi \subset \Pi$ if $\Phi=\{\varphi \in \Pi:\langle\lambda, \varphi\rangle=0\}$. If $\lambda$ is a positive weight then $\pi_{\lambda}$ is the corresponding irreducible C-linear representation of $g, V^{\lambda}$ is the representation space, and $v_{\lambda}$ denotes an arbitrary nonzero highest weight vector. We have the usual map $p: V^{\lambda} \rightarrow \operatorname{proj}\left(V^{\lambda}\right)$ onto the complex projective space determined by $V^{\lambda}$, and $\tilde{\pi}_{\lambda}$ denotes the projective representation of $G$ on $\operatorname{proj}\left(V^{\lambda}\right)$ determined by these data. If $\lambda$ belongs to $\Phi$ we note that $\mathcal{P}_{\Phi}=\left\{h \in \mathcal{G}: \pi_{\lambda}(h)\left(v_{\lambda}\right)\right.$ is a multiple of $\left.v_{\lambda}\right\}$, so $X=G / P_{\Phi}$ is realized as a subvariety of $\operatorname{proj}\left(V^{\lambda}\right)$ which is an orbit, $X=\tilde{\pi}_{\lambda}(G)\left(\mathrm{pv}_{\lambda}\right)$.

3.8. Corollary. Let $X=G / P$ be a complex flag manifold, $x \in X$, $G_{0}$ a real form of $G, \xi \subset \subset \odot_{x}$ a $\tau$-stable Cartan subalgebra of $\mathrm{G}$, and $\Pi$ a simple root system such that $\mathcal{P}_{x}=\mathcal{P}_{\Phi}$ for some $\Phi \subset \Pi$. Then $\operatorname{dim}_{R} G_{0}(x)$ $=\operatorname{dim}_{C} X$ if and only if there is a positive weight $\lambda$ which belongs to $\Phi$, such that 
(i) $V^{\lambda}$ has a $\pi_{\lambda}\left(\mathcal{S}_{0}\right)$-stable real form $V_{\mathbf{R}}^{\lambda}$ and

(ii) $V_{\mathbf{R}}^{\lambda}$ contains a nonzero highest weight vector $v_{\lambda}$.

In that case, identifying $X=\tilde{\pi}_{\lambda}(G)\left(p v_{\lambda}\right)$, the set of real points of $X$ is given by

$$
X_{\mathbf{R}}=X \cap p\left(V_{\mathbf{R}}^{\lambda}\right)=\tilde{\pi}_{\lambda}\left(G_{0}\right)\left(p v_{\lambda}\right) .
$$

We specialize Theorem 3.6 to hermitian symmetric spaces.

3.9. Corollary. Let $X=G / P=G_{u} / K$ be a hermitian symmetric space of compact type, let $X_{0}=G_{0} / K$ be the dual bounded symmetric domain, take the Borel embedding $X_{0} \subset X$ given by $X_{0}=G_{0}\left(x_{0}\right)$ where $P=P_{x_{0}}$ and $K=G_{0} \cap P=G_{u} \cap P$. Let $\zeta$ be the Bergman-Silov boundary of $X_{0}$ in $X$. Then $S$ is the unique closed $G_{0}$-orbit on $X$ and is contained in the closure of every $G_{0}$-orbit. Let $x \in S$ and let $c \in G_{u}$ be the Cayley transform (Koranyi-Wolf [2]) carrying $x_{0}$ to $x$. Then the following conditions are equivalent.

(i) $\operatorname{dim}_{R} \zeta=\operatorname{dim}_{\mathrm{e}} X$.

(ii) $X_{0}$ is a tube domain.

(iii) Viewing $G \operatorname{def} / \mathbf{R}$ with $G_{0}$ as the identity component of $G_{\mathbf{R}}$ we have ad(c) $P$ defined over $\mathbf{R}$.

(iv) $X$ is a complex projective variety defined over $\mathrm{R}$ in such a manner that $\check{S}=X_{\mathbf{R}}$.

Proof. It is known [2] that $\check{S}$ is the unique closed $G_{0}$-orbit on the topological boundary of $X_{0}$ in $X$. Thus $\zeta$ is the unique closed $G_{0^{-}}$ orbit on $X$.

Equivalence of (i), (iii) and (iv) is given by Theorem 3.6; equivalence of (i) and (ii) is given in [2]. Q.E.D.

In view of Corollary 3.9 one might hope to prove that the closed orbit is always the Bergman-Silov boundary of an open orbit. But this is far from the case; Theorem 5.7 will show us that most open orbits do not have any nonconstant holomorphic functions.

In order to specialize Theorem 3.6 to the case where $P$ is a Borel subgroup of $G$ we have to be able to recognize the cases in which $\mathcal{G}$ has a Borel subalgebra $B$ stable under $\tau$. That is the case in which $\mathcal{G}_{0}$ (resp. $G_{0}$ ) is a "Steinberg normal real form" of $\mathcal{G}$ (resp. of $G$ ).

Recall that $g_{0}$ (resp. $G_{0}$ ) is called the Cartan normal real form or normal real form of $\mathcal{G}$ (resp. $G$ ) when the following equivalent conditions hold.

(3.10a) $G_{0}$ has a Cartan subgroup $H_{0}$ that is split/ $\mathbf{R}$, i.e. that is a real vector group, i.e. such that the $\mathfrak{H C}_{0}^{\mathrm{e}}$-roots of $\mathcal{G}$ take real values on $\mathfrak{H}_{0}$.

(3.10b) If $K$ is a maximal compact subgroup of $G_{0}$ then the symmetric space rank of $G_{0} / K$ is equal to the Lie group rank of $G_{0}$. 
(3.10c) $\mathcal{G}_{0}$ is a direct sum of simple algebras from the list:

type $A_{n}:$ Lie algebra of $\operatorname{SL}(n+1, \mathbf{R})$

type $B_{n}:$ Lie algebra of $\operatorname{SO}^{n}(2 n+1)=\operatorname{SO}(n, n+1)$

type $C_{n}$ : Lie algebra of $\mathbf{S p}(n, \mathbf{R})$

type $D_{n}$ : Lie algebra of $\operatorname{SO}^{n}(2 n)=\operatorname{SO}(n, n)$

type $G_{2}$ : maximal compactly embedded subalgebra $A_{1} \oplus A_{1}$

type $F_{4}: \quad$ maximal compactly embedded subalgebra $A_{1} \oplus C_{3}$

type $E_{6}$ : maximal compactly embedded subalgebra $C_{4}$

type $E_{7}:$ maximal compactly embedded subalgebra $A_{7}$

type $E_{8}:$ maximal compactly embedded subalgebra $D_{8}$

The Cartan normal real form of $\mathcal{G}$ is a Steinberg normal real form. For if $H_{0}^{\mathfrak{e}}$ is an R-split Cartan subgroup of $G_{0}$ and $\Delta^{+}$is a positive $\mathcal{F}_{0}^{\mathbf{e}}$-root

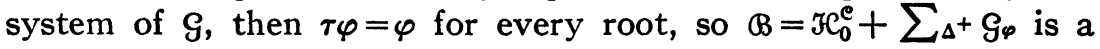
$\tau$-stable Borel subalgebra of $\mathcal{G}$.

Let $\sigma$ be a Cartan involution of $\mathcal{S}_{0}$ and $\mathcal{S}_{0}=\mathfrak{K}+\mathscr{N}$ the Cartan decomposition. Let $\mathcal{H}_{0}=\mathcal{H}_{T}+\mathcal{H}_{V}$ be a $\sigma$-stable Cartan subalgebra of $\mathcal{S}_{0}$, let $\mathfrak{H}=\mathcal{H C}_{0}^{\mathfrak{e}}$, choose a system $\Delta^{+}$of positive $\mathfrak{H}$-roots of $\mathcal{G}$, and let $B=\mathfrak{H}+\sum_{\Delta^{+}} \mathcal{G}_{\varphi}$. If $\tau \mathbb{Q}=\mathbb{B}$ then we have $h \in i \mathcal{H}_{T}+\mathfrak{H}_{V}$ such that

$$
\{\varphi \in \Delta: \varphi(h)>0\}=\Delta^{+}=\{\varphi \in \Delta: \varphi(\tau h)>0\} \text {. }
$$

Then $h=h_{T}+h_{V}, \tau(h)=-h_{T}+h_{V}$, such that

$$
\Delta^{+}=\left\{\varphi \in \Delta^{+}: \varphi\left(h_{V}\right)>0\right\} \text {. }
$$

In other words $\mathcal{H}_{V}$ contains a regular element of $\mathcal{G}$, and thus $\mathcal{H}_{0}$ is a maximally split Cartan subalgebra of $\mathcal{G}_{0}$. Conversely, if $\mathfrak{K}_{V}$ contains a regular element of $\mathcal{G}$, that element defines a $\tau$-stable positive root system $\Delta^{+}$for which $\tau ß=ß$.

Now we can say that $\mathcal{G}_{0}$ (resp. $G_{0}$ ) is a Steinberg normal real form of $\mathcal{G}$ (resp. of $G$ ) if and only if the following equivalent conditions hold. There $\mathfrak{H}_{0}=\mathfrak{J}+Q$ is a maximally split Cartan subalgebra of $\mathcal{G}_{0}$.

(3.11a) $G$ has a Borel subalgebra $B$ with $\tau Q B=B$.

(3.11b) a contains a regular element of $\mathcal{G}$.

(3.11c) Every $\mathfrak{H}_{0}^{\mathrm{e}}$-root has nonzero restriction to $a$.

(3.11d) Let $K$ be a maximal compact subgroup of $G_{0}$ and let $S$ be the Satake diagram of the symmetric space $G_{0} / K$. Then $s$ has no black vertices and $\mathfrak{K}$ contains no simple ideal of $g_{0}$.

(3.11e) $g_{0}$ is a direct sum of simple algebras from the list: 
any type: complex simple Lie algebra

any type: Cartan normal real form

type $A_{2 n-1}$ : Lie algebra of $\mathrm{SU}^{n}(2 n)=\mathrm{SU}(n, n)$

type $D_{n}$ : Lie algebra of $\operatorname{SO}^{n-1}(2 n)=\mathrm{SO}(n-1, n+1)$

type $E_{6}: \quad$ maximal compactly embedded subalgebra $A_{1} \oplus A_{5}$

Summarizing, we have the following special case of Theorem 3.6.

3.12. Corollary. Let $X=G / B$, complex flag manifold where $B$ is a Borel subgroup of $G$. Let $x \in X$ and $G_{0}$ a real form of $G$, such that $G_{0}(x)$ is the closed orbit on $X$. Then the following conditions are equivalent:

(i) $\operatorname{dim}_{R} G_{0}(x)=\operatorname{dim}_{C} X$.

(ii) $G$ is a Steinberg normal real form of $G$.

(iii) Go satisfies (3.11e) above.

4. Open orbits: construction, covering and counting. We work with a complex flag manifold $X=G / P$, a point $x \in X$, a real form $G_{0}$ of $G$, conjugation $\tau$ of $G$ over $G_{0}$, a $\tau$-stable Cartan subalgebra $\Re \subset \mathcal{P}_{x}$ of $\mathcal{G}$, and a system $\Pi$ of simple roots such that $\mathcal{P}_{x}=\mathcal{P}_{\Phi}$ for some $\Phi \subset \Pi$. Theorem 2.12 says that $G_{0}(x)$ is an open $G_{0}$-orbit on $X$ if and only if $\Phi^{u} \cap \tau \Phi^{u}$ is empty.

4.1. Lemma. Let $\mathfrak{H}_{0}$ be a Cartan subalgebra of $\mathrm{Go}$. Choose a maximal compact subgroup $K$ of $G_{0}$, and take the Cartan decomposition $\mathrm{Go}_{0}$ $=\mathfrak{K}+\mathfrak{T}$, such that

$$
\mathfrak{H}_{0}=\mathfrak{H}_{T}+\mathcal{H}_{V}, \quad \mathfrak{H}_{T}=\mathcal{H}_{0} \cap \mathscr{K}, \quad \mathfrak{H}_{V}=\mathfrak{H}_{0} \cap \mathscr{T},
$$

decomposition of $\mathcal{H}_{0}$ into toral and vector parts. Then the following conditions are equivalent:

(i) $\mathfrak{H}_{\boldsymbol{T}}$ is a Cartan subalgebra of $\mathfrak{K}$.

(ii) $\mathfrak{H C}_{T}$ contains a regular element of $\mathcal{G}$.

(iii) $\tau$ sends some Weyl chamber of $\mathfrak{H}^{\mathrm{C}}$ to its negative.

(iv) There is a system $\Delta^{+}$of positive $\mathfrak{F}_{0}^{\mathrm{e}}$-roots, $\Delta^{-}=-\Delta^{+}$, such that $\tau \Delta^{+}=\Delta^{-}$.

Proof. (i) implies (ii) because $\mathfrak{K}$ contains a regular element of $\mathcal{G}$. Let $h_{0} \in \mathfrak{H C}_{T}$ be a regular element of $\mathcal{G}$. Then $\mathfrak{H}_{0}$ is the centralizer of $h_{0}$ in $\mathcal{G}_{0}$, so $\mathcal{H}_{T}$ is its own centralizer in $\mathscr{K}$; this shows that (ii) implies (i).

(iii) and (iv) are equivalent; for if $D \subset i \mathcal{H}_{T}+\mathcal{H}_{V}$ is a Weyl chamber and $\Delta^{+}=\{\varphi \in \Delta: \varphi>0$ on $D\}$ is the corresponding positive root system, then $\tau(D)=-D$ if and only if $\tau\left(\Delta^{+}\right)=\Delta^{-}$.

(i) implies (iv); for if $h_{0} \in \mathcal{H C}_{T}$ is a regular element we have $\tau\left(i h_{0}\right)$ $=-i h_{0}$ and $\left\{\varphi \in \Delta: \varphi\left(i h_{0}\right)>0\right\}$ is a positive root system. Conversely 
if $\mathfrak{H C}_{T}$ contains no regular element we have a root $\varphi$ such that $\varphi\left(\mathfrak{H C}_{T}\right)$ $=0$. Then $\varphi \in \mathfrak{F C}_{V}$, so $\tau(\varphi)=\varphi$, and $\tau \Delta^{+}=\Delta^{-}$is impossible for every positive root system. Thus (iv) implies (i). Q.E.D.

4.3. Definition. A Cartan subalgebra $\mathfrak{H}_{0} C \mathrm{~S}_{0}$ is maximally compact if it satisfies the conditions of Lemma 4.1, is compact if moreover $\mathfrak{H C}_{0}=\mathfrak{H C}_{T}$ in Lemma 4.1, i.e. if rank $K=\operatorname{rank} G_{0}$.

4.4. Lemma. Any two maximally compact Cartan subalgebras of $g_{0}$ are conjugate.

Proof. Let $\mathfrak{H}_{0}$ and $\mathfrak{F C}_{0}^{\prime}$ be maximally compact Cartan subalgebras of $g_{0}$. We have maximal compact subgroups $K, K^{\prime} \subset G_{0}$, Cartan decompositions $\Re+\mathscr{K}=\mathcal{S}_{0}=\mathfrak{K}^{\prime}+\mathfrak{K}^{\prime}$, and expressions $\mathfrak{K}_{0}=\mathfrak{K}_{T}+\mathfrak{K}_{V}$ and $\mathfrak{H C}_{0}^{\prime}=\mathfrak{H C}_{T}^{\prime}+\mathfrak{H C}_{V}^{\prime}$ as in (4.2). By hypothesis $\mathfrak{H C}_{T}$ is a Cartan subalgebra of $\mathcal{K}$ and $\mathcal{K}_{T}^{\prime}$ is a Cartan subalgebra of $\mathcal{K}^{\prime}$.

$G_{0}$ has an element $g$ such that $\operatorname{ad}(g) \mathcal{K}^{\prime}=\mathfrak{K}$, so we may replace $\mathcal{K C}_{0}^{\prime}$ by its conjugate $\operatorname{ad}(g) \mathcal{H}_{0}^{\prime}$ and assume $\mathscr{K}=\mathfrak{K}^{\prime} . K$ has an element $k$ such that $\operatorname{ad}(k) \mathcal{H C}_{T}^{\prime}=\mathcal{F}_{T}$, so we may further replace $\mathcal{F C}_{0}^{\prime}$ by its conjugate $\operatorname{ad}(k) \mathfrak{H C}_{0}^{\prime}$ and assume $\mathfrak{H C}_{T}^{\prime}=\mathfrak{H C}_{T}^{\prime}$. Now $\mathfrak{H}_{0}=\mathfrak{H}_{T}+\mathfrak{H}_{V}$ and $\mathfrak{H C}_{0}^{\prime}$ $=\mathfrak{H C}_{T}+\mathfrak{F C}_{V}^{\prime}$. Let $h_{0} \in \mathfrak{K}_{T}$ be a regular element of $\mathcal{G}$. Then $\mathfrak{K}_{0}=\mathfrak{L}=\mathfrak{H}_{0}^{\prime}$ where $\mathcal{L}$ is the centralizer of $h_{0}$ in $\mathcal{G}_{0}$. Q.E.D.

4.5. Theorem. Let $X=G / P$ be a complex flag manifold, $x \in X$ and $G_{0}$ a real form of $G$. Then the orbit $G_{0}(x)$ is open in $X$ if and only if there exist

(i) a maximally compact Cartan subalgebra $\mathfrak{H}_{0} \subset \mathcal{G}_{0} \cap \odot_{x}$ of $\mathcal{G}_{0}$, and

(ii) a system II of simple $\mathcal{F C}_{0}^{\mathrm{e}}$-roots such that $\tau \Delta^{+}=\Delta^{-}$and $\mathcal{P}_{x}=\mathcal{P}_{\Phi}$ for some $\Phi \subset \Pi$.

If $P_{1} \subset P_{2}$ are parabolic subgroups of $G, X_{i}=G / P_{i}$ the resulting complex flag manifolds, $x_{i} \in X_{i}$ and $\pi: X_{1} \rightarrow X_{2}$ the natural projection, then

(1) if $G_{0}\left(x_{1}\right)$ is an open orbit on $X_{1}$ then $\pi\left(G_{0}\left(x_{1}\right)\right)=G_{0}\left(\pi x_{1}\right)$ is an open orbit on $X_{2}$, and

(2) if $G_{0}\left(x_{2}\right)$ is an open orbit on $X_{2}$ then $\pi^{-1}\left(G_{0}\left(x_{2}\right)\right)$ contains an open $G_{0}$-orbit on $X_{1}$.

Proof. We first prove the assertions on $\pi: X_{1} \rightarrow X_{2}$. If $G_{0}\left(x_{2}\right)$ is open in $X_{2}$ then $\pi^{-1}\left(G_{0}\left(x_{2}\right)\right)$ is open in $X_{1}$ because $\pi$ is continuous. As $G_{0}$ commutes with $\pi, \pi^{-1}\left(G_{0}\left(x_{2}\right)\right)$ is a (necessarily finite) union of $G_{0}$-orbits, so it contains an orbit of full dimension in $X_{1}$; that orbit is necessarily open. This proves assertion (2). If $G_{0}\left(x_{1}\right)$ is open in $X_{1}$ then $\pi\left(G_{0}\left(x_{1}\right)\right)=G_{0}\left(\pi x_{1}\right)$ is open in $X_{2}$ because $\pi$ is an open map. This proves assertion (1).

Suppose for the moment that $P$ is a Borel subgroup of $G$ and let

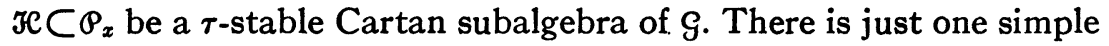


root system $I$ such that $P_{x}=P_{\Phi}$ with $\Phi \subset \Pi ; \Phi$ is empty and $\Pi$ is characterized by $\Phi^{u}=\Delta^{+}$. If $G_{0}(x)$ is open, so $\Phi^{u} \cap \tau \Phi^{u}$ is empty by Theorem 2.12, then we have $\tau \Delta^{+}=\Delta^{-}$, and $\mathcal{H} \mathcal{C}_{0}=\mathcal{H C} \cap \mathcal{G}_{0}$ is a maximally compact Cartan subalgebra of $\mathcal{G}_{0}$ by Lemma 4.1. Conversely, if $\mathfrak{H C}_{0}=\mathfrak{H} \cap \mathcal{G}_{0}$ is maximally compact and $\tau \Delta^{+}=\Delta^{-}$, then $\Phi^{u} \cap \tau \Phi^{u}$ is empty, so $G_{0}(x)$ is open in $X$ by Theorem 2.12 . The first statement is proved for the case where $P$ is a Borel subgroup of $G$.

Now in general choose a Borel subgroup $B \subset P$ of $G$, let $Y=G / B$ be the complex flag manifold, and let $\pi: Y \rightarrow X$ be the natural projection. First suppose $G_{0}(x)$ open in $X$. Then assertion (2), which was already proved, provides an element $y \in \pi^{-1}(x)$ such that $G_{0}(y)$ is open in $Y$. Note $B_{y} \subset \mathcal{P}_{x}$. Choose a $\tau$-stable Cartan subalgebra $\mathfrak{F C} \subset \Re_{y}$ of $\mathcal{G}$ and the simple root system $\Pi$ such that $B_{y}=\mathcal{P}_{\Phi}, \Phi$ empty. As was seen, $\mathfrak{H}_{0}=\mathfrak{H} \cap \mathcal{G}_{0}$ is a maximally compact Cartan subalgebra of $\mathcal{G}_{0}$, and $\tau \Delta^{+}=\Delta^{-}$, because $G_{0}(y)$ is open in $Y$. Now $\mathcal{P}_{x}=\mathcal{P}_{\Psi}$ for some $\Psi \subset I$ because $B_{y} \subset P_{x}$. Thus we have (i) and (ii). Conversely suppose that there is a maximally compact Cartan subalgebra $\mathfrak{H}_{0} \subset \boldsymbol{P}_{x}$ of $\mathcal{G}_{0}$, a system $\Pi$ of simple $\mathcal{F}_{0}^{\mathfrak{e}}$-roots such that $\tau \Delta^{+}=\Delta^{-}$, and a subset $\Psi \subset \Pi$ such that $\mathcal{P}_{x}=\mathcal{P}_{\Psi} . \tau \Delta^{+}=\Delta^{-}$says that $\Delta^{+} \cap \tau \Delta^{+}$is empty. $\Psi \subset I I$ says that $\Psi^{u} \subset \Delta^{+}$, so now $\Psi^{u} \cap \tau \Psi^{u}$ is empty. Theorem 2.12 now implies that $G_{0}(x)$ is open in $X$. Q.E.D.

Now we set out to construct and count the open $G_{0}$-orbits on $X=G / P$. To do this we choose a Borel subgroup $B \subset P$ and let $\pi: Y \rightarrow X$ be the projection, $Y=G / B$. First we construct and count the open $G_{0}$-orbits on $Y$, and then we carry the result down to $X$.

4.6. Theorem. Let $Y=G / B$, complex flag manifold where $B$ is a Borel subgroup of $G$. Let $G_{0}$ be a real form of $G$ and choose a maximally compact Cartan subalgebra $\mathfrak{H}_{0}=\mathfrak{H}_{T}+\mathfrak{H}_{V}$ of $\mathcal{G}_{0}$. Let $\left\{\mathfrak{D}_{1}, \cdots, D_{m}\right\}$ be the chambers in ife $\mathcal{H}_{T}$ cut out by the $\mathcal{H}_{0}^{\mathrm{e}}$-roots of $\mathcal{G}$, and let $W_{K}$ denote the Weyl group of $K$ for the Cartan subalgebra $\mathfrak{H}_{T}$ of $\mathcal{K}$.

1. Let $1 \leqq \alpha \leqq m$, choose $h_{\alpha} \in D_{\alpha}$, let $\Pi_{\alpha}$ be the system of simple $\mathfrak{F}_{0}^{\mathrm{e}}$ roots of $\mathcal{G}$ for the positive root system $\Delta_{\alpha}^{+}=\left\{\varphi \in \Delta: \varphi\left(h_{\alpha}\right)>0\right\}$, let $\mathrm{B}_{\alpha}=\mathfrak{F C}_{0}^{\mathrm{e}}+\sum_{\Delta_{\alpha}^{+}} \mathcal{G}_{\varphi}$ be the corresponding Borel subalgebra of $\mathcal{G}$ and let $y_{\alpha} \in Y$ be the element such that $\wp_{\nu_{\alpha}}=B_{\alpha}$. Then the open $G_{0}$-orbits on $Y$ are just the $G_{0}\left(y_{\alpha}\right)$, and $G_{0}\left(y_{\alpha}\right)=G_{0}\left(y_{\beta}\right)$ if and only if some element of $W_{K}$ sends $D_{\alpha}$ to $D_{\beta}$.

2. $W_{K}$ acts simply on $\left\{D_{1}, \cdots, D_{m}\right\}$.

4 Given a root $\varphi,\left\{h \in i \mathcal{C}_{T}: \varphi(h)=0\right\}$ is a subspace of codimension $\geqq 1$ in $i \mathcal{J}_{T}$. The union of these subspaces, as $\varphi$ ranges over the root system $\Delta$, is the intersection of $i \mathcal{C}_{T}$ with the "diagram" in $i \mathcal{H C}_{T}+\mathcal{H C}_{V}$. The $D_{\alpha}$ are the components of the complement of this intersection in $i \mathcal{F}_{T}$, i.e. of the set of regular elements of $\mathcal{G}$ contained in $i \mathcal{H}_{T}$. 
3. There are precisely $m /\left|W_{K}\right|$ distinct open $G_{0}$-orbits on $Y$.

Proof. Let $G_{0}(y)$ be an open orbit on $Y$. Theorem 4.5(i) gives us a maximally compact Cartan subalgebra $\mathcal{H}_{0}^{\prime} \subset B_{y}$ of $\mathcal{S}_{0}$ and Lemma 4.4 gives us an element $g \in G_{0}$ such that $\operatorname{ad}(g) \mathcal{H}_{0}^{\prime}=\mathfrak{H}_{0}$. Replacing $y$ by $g(y), G_{0}(y)$ is not changed but we may assume $\mathfrak{H}_{0} \subset \Re_{y}$. Theorem 4.5(ii) provides a simple root system $\Pi$, and the corresponding system $\Delta^{+}$of positive roots, such that $\tau \Delta^{+}=\Delta^{-}$and $B_{y}=\mathcal{H}_{0}^{e}+\sum_{\Delta^{+}} \mathcal{G}_{\varphi}$. Let $h \in i \mathcal{F}_{T}+\mathcal{H}_{V}$ be a regular element such that $\Delta^{+}=\{\varphi \in \Delta: \varphi(h)>0\}$. Decompose $h=h_{T}+h_{V}, h_{T} \in i \mathcal{K}_{T}$ and $h_{V} \in \mathcal{H C}_{V}$, so $\varphi(h)=\varphi\left(h_{T}\right)+\varphi\left(h_{V}\right)$ and $(\tau \varphi)(h)=\varphi(\tau h)=-\varphi\left(h_{T}\right)+\varphi\left(h_{V}\right)$ for $\varphi \in \Delta$. Now let $\varphi \in \Delta^{+}$and use $\tau \Delta^{+}=\Delta^{-}$; so $\varphi\left(h_{T}\right)+\varphi\left(h_{V}\right)>0>-\varphi\left(h_{T}\right)+\varphi\left(h_{V}\right)$, which implies $\varphi\left(h_{T}\right)>0$. Thus $\Delta^{+}=\Delta_{\alpha}^{+}$, so $B_{y}=B_{\alpha}$, so $y=y_{\alpha}$, so $G_{0}(y)=G_{0}\left(y_{\alpha}\right)$, for the index $\alpha$ with $h_{T} \in D_{\alpha}$. This proves that every open $G_{0}$-orbit on $Y$ is one of the $G_{0}\left(y_{\alpha}\right)$. Conversely, $\tau\left(h_{\alpha}\right)=-h_{\alpha}$, which implies $\tau \Delta_{\alpha}^{+}=\Delta_{\alpha}^{-}$, so $G_{0}\left(y_{\alpha}\right)$ is an open orbit.

Let $w \in W_{K}$ send $D_{\alpha}$ to $D_{\beta}$. Represent $w=\operatorname{ad}(k) \mid \mathfrak{H}_{0}^{\mathfrak{e}}, k \in K$. Now $\operatorname{ad}(k) B_{\alpha}=\bigotimes_{\beta}$, i.e. $k\left(y_{\alpha}\right)=y_{\beta}$, so $G_{0}\left(y_{\beta}\right)=G_{0}\left(k y_{\alpha}\right)=\left(G_{0} k\right)\left(y_{\alpha}\right)=G_{0}\left(y_{\alpha}\right)$. Conversely suppose $G_{0}\left(y_{\alpha}\right)=G_{0}\left(y_{\beta}\right)$, so $g\left(y_{\alpha}\right)=y_{\beta}$ for some $g \in G_{0}$. $\mathfrak{F C}_{0}$ is both the centralizer of $y_{\alpha}$ in $\mathcal{G}_{0}$ and the centralizer of $y_{\beta}$ in $\mathcal{G}_{0}$; now $\operatorname{ad}(g) \mathcal{C}_{0}=\mathcal{H}_{0}$ and $\operatorname{ad}(g) B_{a}=B_{\beta}$. Thus $g$ represents an element $w$ of the Weyl group $W_{G_{0}}$ of $G_{0}$ relative to $\mathcal{H}_{0}$, such that $w\left(D_{\alpha}\right)=\mathscr{D}_{\beta}$. The analytic subgroup for $\mathfrak{H}_{0}$ in $G_{0}$ has finite index in its normalizer, for the latter is algebraic and thus has only finitely many components. It follows that $g=k v$ where $k \in K$ represents $w$ and $v \in \exp \left(\mathcal{H C}_{V}\right)$. In other words $w \in W_{K}$. We have proved that $G_{0}\left(y_{\alpha}\right)=G_{0}\left(y_{\beta}\right)$ if and only if an element of $W_{K}$ sends $D_{\alpha}$ to $D_{\beta}$.

Let $w \in W_{K}$ such that $w\left(D_{\alpha}\right)=\mathfrak{D}_{\alpha}$. As $W_{K} \subset W_{G}$, we have $w \in W_{G}$. $D_{\alpha}=D \cap i \mathcal{C}_{T}$ for some Weyl chamber $D \subset i \mathcal{C}_{T}+\mathfrak{H C}_{V}$; as $\mathfrak{D}_{\alpha}$ is nonempty now $w(D)=D$. That says $w=1$. Now $W_{K}$ acts simply on $\left\{D_{1}, \cdots, D_{m}\right\}$.

Now we can count the open $G_{0}$-orbits. They are the $G_{0}\left(y_{\alpha}\right)$, $1 \leqq \alpha \leqq m$, and the action of $W_{K}$ partitions them into sets of $\left|W_{K}\right|$ elements under the relation of $W_{K}$-equivalence. Thus there are $m /\left|W_{K}\right|$ distinct open orbits. Q.E.D.

If rank $K=\operatorname{rank} G_{0}$, i.e. if $\mathfrak{H}_{0}$ is a compact Cartan subalgebra of $\mathcal{G}_{0}$ in the notation of Theorem 4.6, then $\mathfrak{H C}_{0}=\mathfrak{H}_{T}$ and the $\mathscr{D}_{\alpha}$ are the Weyl chambers. Then the number $m$ of $D_{\alpha}$ is the order $\left|W_{G}\right|$ of the Weyl group of $G$, so part 3 of Theorem 4.6 reduces to

4.7. Corollary. Let $Y=G / B$, complex flag manifold where $B$ is a Borel subgroup of $G$. Let $G_{0}$ be a real form of $G$ whose maximal compact subgroup $K$ satisfies rank $K=$ rank $G_{0}$. Then there are precisely $\left|W_{G}\right| /\left|W_{K}\right|$ distinct open $G_{0}$-orbits on $Y$. 
On the other hand, if rank $K<$ rank $G_{0}$, there may be Weyl chambers in $i \mathfrak{F}_{T}+\mathfrak{H C}_{V}$ which do not meet $i \mathfrak{F C}_{T}$, so the number of $\mathbb{D}_{\alpha}$ may be less than $\left|W_{G}\right|$. For example, if $G=\operatorname{SL}(3, \mathbf{C})$ and $G_{0}=\mathbf{S L}(3, \mathbf{R})$, then $\left|W_{G}\right|=6$, so there are 6 Weyl chambers, but 4 of them are not sent to their negatives by $\tau$; thus $m=2,\left|W_{K}\right|=\left|W_{\text {So(3) }}\right|=2$, and so there is just one open $G_{0}$-orbit on $G / B$. This trick for finding $m$ works in general:

4.8. Corollary. Let $Y=G / B$, complex flag manifold where $B$ is a Borel subgroup of $G$. Let $G_{0}$ be a real form of $G$, let $K$ be a maximal compact subgroup of $G_{0}$, let $\sigma$ denote the involutive automorphism of $\mathcal{G}_{0}$ with fixed point set $\mathfrak{K}$, and let $\mathfrak{K}_{0}=\mathfrak{K}_{T}+\mathfrak{K C}_{V}$ be a maximally compact Cartan subalgebra of $\mathcal{S}_{0}$ such that $\mathfrak{H}_{T} \subset \Re$. Define

$$
\begin{aligned}
& W_{G}: \text { Weyl group of } G \text { for the Cartan subalgebra } \mathfrak{F C}_{0}^{\mathfrak{e}} \text {, } \\
& W_{K} \text { : Weyl group of } K \text { for the Cartan subalgebra } \mathfrak{F}_{T} \text {, } \\
& W_{G}^{\mathfrak{F}_{0}}: \quad\left\{w \in W_{G}: w\left(\mathfrak{F C}_{0}\right)=\mathfrak{F C}_{0}\right\}, \text { subgroup of } W_{G} .
\end{aligned}
$$

Then $W_{G}^{3 C_{0}}$ is simply transitive on the set of Weyl chambers which meet ${ }^{3 \mathcal{C}_{T}}$ (i.e. which are sent to their negatives by $\tau$ ), $W_{G}^{\Im C_{0}}$ is the centralizer of $\sigma \mid{ }_{i C_{T}+\mathcal{C O}_{\mathrm{V}}}$ in $W_{G}$, and $G_{0}$ has precisely $\left|W_{G}^{\mathrm{SC}_{0}}\right| /\left|W_{K}\right|$ distinct open orbits on $Y$.

PRoof. Let $D$ be a Weyl chamber which meets $i \mathcal{F}_{T}$, i.e. such that $\tau \mathscr{D}=-\mathfrak{D}$. Note that $\sigma=-\tau$ on the ambient space $i \mathcal{C C}_{T}+\mathfrak{H C}_{V}$ of the Weyl chambers. Let $w \in W_{G}$ and notice $-w(D)=w(\tau D)$, so $\tau(w D)$ $=-w D$ if and only if $w D=\tau^{-1} w \tau D$, i.e. if and only if $w \tau=\tau w$, i.e. if and only if $w \sigma=\sigma w$, i.e. if and only if $w$ preserves $i \mathfrak{K}_{T}$ and $\mathfrak{F C}_{V}$, i.e. if and only if $w \in W_{G}^{s C_{0}}$. Now our assertions follow from part 3 of Theorem 4.6. Q.E.D.

Now we push Theorem 4.6 down to a general complex flag manifold.

4.9. TheOREM. Let $X=G / P$ be a complex flag manifold, let $B \subset P$ be a Borel subgroup of $G$, let $Y=G / B$, and let $\pi: Y \rightarrow X$ be the natural projection. Let $G_{0}$ be a real form of $G$, let $x \in X$ such that $G_{0}(x)$ is open in $X$, and let $\mathcal{H}_{0} \subset \mathcal{S}_{0} \cap \mathcal{P}_{x}$ be a maximally compact Cartan subalgebra of So. Decompose $\mathcal{P}_{x}=\mathcal{P}_{x}^{r}+\mathcal{P}_{x}^{u}$ relative to $\mathfrak{H}=\mathfrak{F}_{0}^{e}$ and define $\mathcal{P}_{x}^{-u}=\sum_{-\Phi^{u}} \complement_{\varphi}$.

1. Let $y \in \pi^{-1}(x)$. Then $G_{0}(y)$ is open in $Y$ if and only if $B_{y}=B^{\prime}$ $+\left(\mathcal{P}_{x}^{r} \cap \tau \mathcal{P}_{x}^{-u}\right)+\mathscr{P}_{x}^{u}$ where $\mathbb{B}^{\prime}$ is a Borel subalgebra of $\mathcal{P}_{x}^{r} \cap \tau \mathcal{P}_{x}^{r}$ $=\left(\mathcal{P}_{x} \cap \tau \mathcal{P}_{x}\right)^{r}$ such that $\mathbb{B}^{\prime} \cap \tau \mathbb{B}^{\prime}=\mathfrak{K C}$.

2. The open $G_{0}$-orbits in $Y$ which lie over $G_{0}(x)$ are in one to one correspondence with the open $\left(P_{x}^{r} \cap \tau P_{x}^{r}\right)$-orbits on $\left(P_{x}^{r} \cap \tau P_{x}^{r}\right) /($ Borel $)$.

3. The open $G_{0}$-orbits on $X$ are enumerated by the elements of the

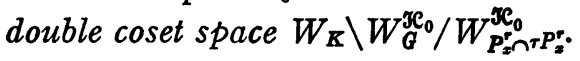


PRoof. Let $y \in \pi^{-1}(x)$ with $G_{0}(y)$ open in $Y$. We may assume $\mathfrak{H C} \subset \mathbb{B}_{y}$. Then $\mathscr{Q}_{y}=\mathbb{B}^{\prime \prime}+\mathbb{P}_{x}^{u}=\mathfrak{H C}+\sum_{\Delta+} \mathcal{S}_{\varphi}$ where $\Delta^{+}$is a positive root system such that $\Phi^{u} C \Delta^{+}$and $\tau \Delta^{+}=\Delta^{-}$, and where $\mathscr{B}^{\prime \prime}$ is a Borel subalgebra of $\mathcal{P}_{x}^{r}$. Decompose $\mathcal{P}_{x}^{r}$ as the sum of $\left(\mathcal{P}_{x}^{r} \cap \tau \mathcal{P}_{x}^{r}\right),\left(\mathcal{P}_{x}^{r} \cap \tau \mathcal{P}_{x}^{u}\right)$ and $\left(\mathcal{P}_{x}^{r} \cap \tau \mathcal{P}_{x}^{-u}\right)$. $\Phi^{u} \subset \Delta^{+}$and $\tau \Delta^{+}=\Delta^{-}$imply $\tau \Phi^{u} \subset \Delta^{-}$, so $\mathbb{B}^{\prime \prime}=\mathbb{B}^{\prime}$ $+\left(\mathscr{P}_{x}^{r} \cap \tau \mathscr{P}_{x}^{-u}\right)$ where $\mathscr{B}^{\prime}$ is a Borel subalgebra of $\mathcal{P}_{x}^{r} \cap \tau \mathscr{P}_{x}^{r}$. Now $\tau \Delta^{+}=\Delta^{-}$implies $B^{\prime} \cap \tau B^{\prime}=\mathscr{H}$.

Conversely let $y \in \pi^{-1}(x)$ with $\mathbb{B}_{y}=\mathbb{B}^{\prime}+\left(\mathcal{P}_{x}^{r} \cap \tau \mathcal{P}_{x}^{-u}\right)+\mathcal{P}_{x}^{u}$ where $\mathbb{B}^{\prime}$ is a Borel subalgebra of $\mathscr{P}_{x}^{r} \cap \tau \mathcal{P}_{x}^{r}$ such that $\mathbb{B}^{\prime} \cap \tau \mathbb{B}^{\prime}=\mathfrak{H}$. Then $\mathbb{B}_{y} \cap \tau \mathbb{B}_{y}=\mathcal{K}$, so $G_{0}(y)$ is open in $Y$. This completes the proof of (1).

The number of distinct Borel subalgebras $\mathbb{B}^{\prime}+\left(\mathcal{P}_{x}^{r} \cap \tau \mathcal{P}_{x}^{-u}\right)+\mathcal{P}_{x}^{u}$, $\mathbb{B}^{\prime} \subset \mathscr{P}_{x}^{\prime} \cap \tau \mathcal{P}_{x}^{x}$ Borel subalgebra, $\mathbb{B}^{\prime} \cap \tau \mathbb{B}^{\prime}=\mathfrak{F}$, is the number of chambers cut out on $i \mathcal{F}_{T}$ by the $\mathcal{H}$-roots of $\mathcal{P}_{x}^{r} \cap \tau \mathcal{P}_{x}^{r}=\left(\mathcal{P}_{x} \cap \tau \mathcal{P}_{x}\right)^{r}$. Now, as in Theorem 4.6(3), the number of open $G_{0}$-orbits on $Y$ over $G_{0}(x)$ is the number of open $\left(P_{x}^{r} \cap \tau P_{x}^{r}\right)$-orbits on $\left(P_{x}^{r} \cap \tau P_{x}^{r}\right) /($ Borel $)$.

Finally we count the open $G_{0}$-orbits on $X$. Corollary 4.8 identifies the coset space $W_{K} \backslash W_{G}{ }^{3 C_{0}}$ with the open $G_{0}$-orbits on $Y$ as follows. Choose a Weyl chamber $\mathbb{D} C i \mathcal{C}_{T}+\mathfrak{H C}_{V}$ such that $\tau \mathscr{D}=-\mathbb{D}$. Then all other such Weyl chambers are given by the $w(D), w \in W_{G}^{30}$; and the Borel subalgebras specified by $w_{1}(D)$ and $w_{2}(D), w_{i} \in W_{G}^{3 C_{0}}$, give the same open $G_{0}$-orbit if and only if $w_{1}=w_{K} w_{2}$ for some $w_{K} \in W_{K}$. On the other hand, if $D$ specifies one of the $\mathbb{B}^{\prime}+\left(\mathcal{P}^{r} \cap \tau \mathcal{P}_{x}^{-u}\right)+\mathcal{P}_{x}^{u}$ of (1), all other such $D$ are given by the $w(D), w \in W_{P_{n}^{*} \sim P P r}^{3 e_{0}}$; this follows from (1) and Corollary 4.8. Now (3) follows. Q.E.D.

5. Open orbits : coset space structure and holomorphic functions. In order to study open orbits as coset spaces of the real group, and in order to study holomorphic functions on open orbits, we need a remark on compact subvarieties:

5.1. Lemma. Let $X=G / P$ be a complex flag manifold, $G_{0}$ a real form of $G, x \in X$, such that $G_{0}(x)$ is open in $X$, and $K$ a maximal compact subgroup of $G_{0}$ such that $G_{0} \cap \beta_{x}$ contains a maximally compact Cartan subalgebra $\mathfrak{K}_{0}=\mathfrak{K}_{T}+\mathfrak{H}_{V}$ of $\mathcal{S}_{0}$ with $\mathfrak{K}_{T} \subset \mathfrak{K}$. Then $K(x)$ is a compact complex submanifold of $G_{0}(x)$.

Proof. Let $B C P$ be a Borel subgroup of $G$, let $Y=G / B$ and $\pi: Y \rightarrow X$ the natural projection, and choose $y \in \pi^{-1}(x)$ such that $G_{0}(y)$ is open in $Y$ and $\mathfrak{F C}_{0}=\bigotimes_{y} \cap \tau \mathfrak{B}_{y}$. Now $\pi(K(y))=K(x)$, and both $K(y)$ and $K(x)$ are compact real submanifolds. If $K(y)$ is a complex submanifold of $G_{0}(y)$, then its every tangent space $K(y)_{k(y)}$ is stable under the almost complex structure operator on $Y$, so $K(x)_{k(x)}$ is also stable for all $k \in K$, proving that $K(x)$ is a complex submanifold of $X$. Now the lemma is reduced to the case where $P$ is a Borel subgroup of $G$. 
Let $P$ be a Borel subgroup. Then $\mathscr{P}_{x} \cap \tau \mathcal{P}_{x}=\mathcal{J C}_{0}^{e}$ because $G_{0}(x)$ is open in $X$. At $x$ the $(\sqrt{-1})$-eigenspace of the almost complex structure is the holomorphic tangent space $\sum_{\Delta^{+}} \mathcal{G}_{-\varphi}$, and the $(-\sqrt{-1})$ eigenspace is the antiholomorphic tangent space $\sum_{\Delta^{+}} \mathcal{G}_{\varphi}$, where $P_{x}$ $=\mathfrak{H C}_{0}^{\mathfrak{e}}+\sum_{\Delta^{+}} \mathcal{G}_{\varphi}$. Now choose $h_{0} \in i \mathfrak{F C}_{T}^{\mathfrak{e}}$ such that $\Delta^{+}=\left\{\varphi \in \Delta: \varphi\left(h_{0}\right)>0\right\}$. Decompose $\mathfrak{K}=\mathfrak{H C}_{T}+\mathcal{F}$ where $\mathfrak{F}$ is the orthocomplement under the Killing form of $\mathfrak{K}$. Now $\mathfrak{F}^{\mathfrak{e}}$ is the sum of the $\mathfrak{F C}^{\mathfrak{e}}{ }_{\mathrm{T}}$-root spaces on $\mathfrak{K}^{\mathfrak{e}}$, $\mathcal{F}^{\mathfrak{e}}$ gives the complexified tangent space to $K(x)$ at $x$, and $\mathscr{F}^{\mathfrak{e}}$ is the sum of its intersections with the holomorphic and antiholomorphic tangent spaces. It follows (look at $h_{0}$ ) that the real tangent space to $K(x)$ at $x$, represented by $\mathcal{F}$, is stable under the almost complex structure. Thus $K(x)$ is a complex submanifold. Q.E.D.

To describe open orbits as coset spaces we need some notation. Let $X=G / P$ be a complex flag manifold, $G_{0}$ a real form of $G, x \in X$, and $\mathfrak{H C} \subset \mathcal{P}_{x}$ a $\tau$-stable Cartan subalgebra of $\mathcal{G}$. For a subset $\Phi$ of a simple KC-root system II we have $\mathcal{P}_{x}=\mathcal{P}_{\Phi}$. Write $\mathcal{P}_{x}^{r}$ for $\mathcal{P}_{\Phi}^{r}$ and $\mathcal{P}_{x}^{u}$ for $\mathcal{P}_{\Phi}^{u}$. Recall, from Lemma 2.10 , that $\odot_{x} \cap \tau \odot_{x}$ is the semidirect sum $\left(\mathcal{P}_{x} \cap \tau \mathcal{P}_{x}^{u}\right)^{r}+\left(\mathcal{P}_{x} \cap \tau \mathcal{P}_{x}^{u}\right)^{u}$ where $\left(\mathcal{P}_{x} \cap \tau \mathcal{P}_{x}\right)^{r}=\mathcal{P}_{x}^{r} \cap \tau \mathcal{P}_{x}^{r}$ and $\left(\mathcal{P}_{x} \cap \tau \mathcal{P}_{x}\right)^{u}$ $=\left(\mathcal{\odot}_{x}^{r} \cap \tau \mathcal{\odot}^{u}\right)+\left(\mathcal{P}_{x}^{r} \cap \tau \mathcal{P}_{x}^{r}\right)+\left(\mathcal{P}_{x}^{u} \cap \tau \mathcal{P}_{x}^{u}\right)$. We define real forms of $\left(\mathcal{P}_{x} \cap \tau \mathcal{P}_{x}\right),\left(\mathcal{P}_{x} \cap \tau \mathcal{P}_{x}\right)^{r}$ and $\left(\mathcal{P}_{x} \cap \tau \mathcal{P}_{x}\right)^{u}$ in the obvious manner, by

$$
\begin{aligned}
& \left(\mathcal{P}_{x} \cap \tau \mathcal{P}_{x}\right)_{0}=\left(\mathcal{P}_{x} \cap \tau \mathcal{P}_{x}\right) \cap \mathcal{S}_{0}, \quad \text { real form of } \mathcal{P}_{x} \cap \tau \mathcal{P}_{x} ; \\
& \left(\mathcal{P}_{x} \cap \tau \mathcal{P}_{x}\right)_{0}^{r}=\left(\mathcal{P}_{x}^{r} \cap \tau \mathscr{P}_{x}^{r}\right) \cap \mathcal{G}_{0}, \quad \text { real form of }\left(\mathcal{P}_{x} \cap \tau \mathcal{P}_{x}\right)^{r} \text {; }
\end{aligned}
$$

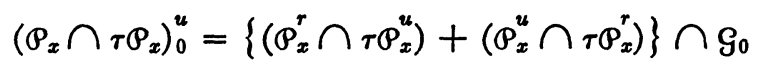

$$
\begin{aligned}
& +\left(\odot_{x}^{u} \cap \tau \mathcal{P}_{x}^{u}\right) \cap g_{0}, \text { real form of }\left(\boldsymbol{P}_{x} \cap \tau \odot_{x}\right)^{u} .
\end{aligned}
$$

Thus $\left(\mathcal{P}_{x} \cap \tau \mathcal{P}_{x}\right)_{0}=\left(\mathcal{P}_{x} \cap \tau \mathscr{P}_{x}\right)_{0}^{r}+\left(\mathcal{P}_{x} \cap \tau \mathcal{P}_{x}\right)_{0}^{u}$ semidirect sum of reductive and nilpotent parts. Rather than proliferate notation, we denote

$$
\left(P_{x} \cap \tau P_{x}\right)_{0} \text { : analytic subgroup of } G_{0} \text { for }\left(\mathcal{P}_{x} \cap \tau \mathcal{P}_{x}\right)_{0},
$$

$$
\left(P_{x} \cap \tau P_{x}\right)_{0}^{r} \text { : analytic subgroup of } G_{0} \text { for }\left(P_{x} \cap \tau \mathcal{P}_{x}\right)_{0}^{r} \text {, }
$$

$$
\left(P_{x} \cap \tau P_{x}\right)_{0}^{u} \text { : analytic subgroup of } G_{0} \text { for }\left(\mathcal{P}_{x} \cap \tau \mathcal{P}_{x}\right)_{0}^{u} \text {. }
$$

These are all closed connected subgroups of $G_{0}$, and $\left(P_{x} \cap \tau P_{x}\right)_{0}$ $=\left(P_{x} \cap \tau P_{x}\right)_{0}^{\tau} \cdot\left(P_{x} \cap \tau P_{x}\right)_{0}^{u}$ semidirect product of reductive and unipotent parts. By definition, $\left(P_{x} \cap \tau P_{x}\right)_{0}$ is the identity component of the isotropy subgroup $G_{0} \cap P_{x}$ of $G_{0}$ at $x$.

5.4. Theorem. Let $X=G / P$ be a complex flag manifold, $G_{0}$ a real form of $G, x \in X$, such that $G_{0}(x)$ is open in $X$. Then the open orbit $G_{0}(x)$ is simply connected and $G_{0}$ has connected isotropy subgroup $\left(P_{x} \cap \tau P_{x}\right)_{0}$ at $x$. 
Proof. We work with a $\tau$-stable Cartan subalgebra $\mathfrak{H} \subset \boldsymbol{P}_{x}$ of $\mathcal{g}$ such that $\mathcal{H C}_{0}=\mathcal{H} \cap \mathcal{G}_{0}$ is a maximally compact Cartan subalgebra of 9o. Now $\mathcal{P}_{x}=\mathcal{P}_{\Phi}$ as usual. Let $\mathfrak{F}_{0}=\mathfrak{H}_{T}+\mathfrak{H C}_{V}, \mathfrak{H C}_{T} \subset \mathcal{K}$, where $K$ is a maximal compact subgroup of $G_{0}$. For the moment let $L$ denote the isotropy subgroup of $G_{0}$ at $x$, so $L \cap K$ is the isotropy subgroup of $K$. Lemma 5.1 gives $K$-invariant complex structure on $K /(L \cap K)$ via its realization as $K(x)$. As $\operatorname{rank}(L \cap K)=\operatorname{rank} K$ because $\mathfrak{H}_{T} \subset \mathscr{L}$, it follows [14] that $L \cap K$ is connected and $K /(L \cap K)$ is simply connected. But $G_{0}(x) \cong G_{0} / L$ has a deformation retraction back onto $K /(L \cap K)$; thus $G_{0}(x)$ is simply connected and it follows that $L$ is connected. Q.E.D.

Let $X=G / P$ be a complex flag manifold and $G_{0}$ a real form of $G$. Then $\mathcal{S}_{0}=\mathcal{S}_{0}^{(1)} \oplus \cdots \oplus \mathcal{S}_{0}^{(i)}$, direct sum of simple ideals, and we have

$$
\begin{aligned}
& \mathcal{S}=\mathcal{S}^{(1)} \oplus \cdots \oplus \mathcal{S}^{(t)} \text { where } \mathcal{g}^{(i)}=\mathcal{G}_{0}^{(i) \mathrm{e}}, \\
& \mathcal{P}=\mathcal{\rho}^{(1)} \oplus \cdots \oplus \mathcal{\rho}^{(t)} \text { where } \rho^{(i)}=\rho \cap \mathcal{g}^{(i)}, \\
& X=X_{1} \times \cdots \times X_{t} \text { where } X_{i}=G^{(i)} / P^{(i)} .
\end{aligned}
$$

Let $x \in X, x=\left(x_{1}, \cdots, x_{i}\right)$ with $x_{i} \in X_{i}$. Then

$$
G_{0}(x)=G_{0}^{(1)}\left(x_{1}\right) \times \cdots \times G_{0}^{(t)}\left(x_{i}\right), \quad G^{(i)}\left(x_{i}\right) \subset X_{i} .
$$

Of course $G_{0}(x)$ is open in $X$ if and only if $G_{0}^{(i)}\left(x_{i}\right)$ is open in $X_{i}$ for $1 \leqq i \leqq t$. Now consider the conditions

(5.5a) $\left(P_{x_{i}}^{(t)} \cap \tau P_{x_{i}}^{(t)}\right)_{0}$ is compact; hence contained in a maximal compact subgroup $K^{(i)}$ of $G_{o}^{(i)}$,

(5.5b) $G_{o}^{(\text {() }} / K^{(i)}$ is a hermitian symmetric coset space,

(5.5c) $G_{o}^{(i)} /\left(P_{x_{i}}^{(i)} \cap \tau P_{x_{i}}^{(i)}\right)_{0} \rightarrow G_{o}^{(i)} / K^{(i)}$ is holomorphic for one of the two invariant complex structures on $G_{o}^{(i)} / K^{(i)}$.

5.6. Definition. Let $G_{0}(x)$ be open in $X$. Then the bounded symmetric domain subordinate to the orbit $G_{0}(x)$ is the coset space

$$
D\left(G_{0}, x\right)=G_{0}^{(1)} / L^{(1)} \times \cdots \times G_{0}^{(t)} / L^{(t)}
$$

where

(i) if the conditions (5.5) all hold, then $L^{(i)}=K^{(i)}$ and $G_{0}^{(i)} / L^{(i)}$ carries the invariant complex structure such that $G_{0}^{(i)} /\left(P_{x_{i}}^{(i)} \cap \tau P_{x_{i}}^{(i)}\right)_{0}$ $\rightarrow G_{0}^{(i)} / L_{0}^{(i)}$ is holomorphic,

(ii) if the conditions (5.5) do not all hold then $L^{(i)}=G_{0}^{(i)}$.

We justify the terminology by observing that

(a) if $G_{0}^{(\text {() }} / L^{(i)}$ is not a point, then it is an irreducible hermitian symmetric space of noncompact type, so $D\left(G_{0}, x\right)$ is a hermitian symmetric space of noncompact type (and possibly of dimension 0 ), and 
(b) $D\left(G_{0}, x\right)$ is the "largest" hermitian symmetric space of noncompact type such that the natural map $G_{0}(x) \rightarrow D\left(G_{0}, x\right)$ is $G_{0}$ equivariant and holomorphic.

5.7. TheOREM. Let $X=G / P$ be a complex fag manifold, $G_{0}$ a real form of $G$, and $x \in X$ such that $G_{0}(x)$ is open in $X$. Let

$$
\psi: G_{0}(x) \rightarrow D\left(G_{0}, x\right)
$$

denote the natural projection onto the bounded symmetric domain subordinate to $G_{0}(x)$. Then the holomorphic functions on $G_{0}(x)$ are just the functions $\tilde{f}=f \cdot \psi$ where $f$ is a holomorphic function on $D\left(G_{0}, x\right)$.

Proof. We first consider the case where $G_{0}$ is simple. Let $\mathfrak{K C}_{0}=\mathfrak{K C}_{T}$ $+\mathcal{H}_{V} \subset \mathcal{S}_{0} \cap \mathcal{P}_{x}$ be a maximally compact Cartan subalgebra of $\mathcal{S}_{0}$; we have $\mathscr{P}_{x}^{x}$ specified by $\mathfrak{H}=\mathfrak{H C}_{0}^{C}$. Let $K$ be a maximal compact subgroup of $G_{0}$ such that $\mathcal{H}_{T} \subset \mathcal{K}$. If $\delta \subset \mathcal{G}, z \in X$ and $h$ is a smooth function on a neighborhood of $z$, then $S(h)_{z}$ denotes the set of values of derivatives of $h$ at $z$ by vector fields induced from elements of $s$.

Suppose that $h$ is a nonconstant holomorphic function on $G_{0}(x)$. As $K(x)$ is a compact complex submanifold of $G_{0}(x)$ by Lemma 5.1, $h$ must be constant on $K(x)$, so $\mathscr{K}(h)_{x}=0$. The vector fields induced by $\left(\mathcal{P}_{x} \cap \tau \mathcal{P}_{x}\right)_{0}$ vanish at $x$, so also $\left(\mathcal{P}_{x} \cap \tau \mathcal{P}_{x}\right)_{0}(h)_{x}=0$. If $\left(P_{x} \cap \tau P_{x}\right)_{0} \nsubseteq K$ then $\mathscr{K}$ and $\left(\mathcal{P}_{x} \cap \tau \mathcal{P}_{x}\right)_{0}$ generate $\mathcal{G}_{0}$, for $\mathcal{G}_{0}$ is simple, so $\mathcal{S}_{0}(h)_{x}=0$; then the same argument shows $\mathcal{G}_{0}(h)_{z}=0$ for every $z \in G_{0}(x)$, so all first derivatives of $h$ vanish, whence $h$ is constant contrary to hypothesis. Thus $\left(P_{x} \cap \tau P_{x}\right)_{0}$ is compact and contained in $K$.

The compactness just proved shows $\mathfrak{H}_{0}=\mathfrak{H}_{T} \subset \mathfrak{K}$. Now the root system $\Delta=\Delta_{K} \cup \Delta_{M}$ where $\Delta_{K}$ is the root system of $\Re^{\mathfrak{e}}$ (compact roots) and $\Delta_{M}$ consists of the remaining roots (noncompact roots). If $\varphi \in \Delta$ then $\tau(\varphi)=-\varphi$ because $\mathcal{H}_{0}$ is compact. Thus $\tau \Phi^{r}=\Phi^{r}$ and $\tau \Phi^{u}=-\Phi^{u}$

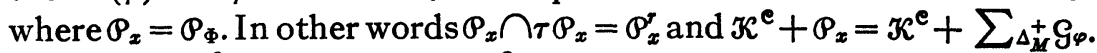
Define $Q=\varkappa^{\mathfrak{e}}+\mathcal{P}_{x}$. We have $\mathfrak{K}^{\mathfrak{e}}(h)_{x}=0$ because $h$ is holomorphic and $\mathscr{K}(h)_{x}=0$; we have $\mathcal{P}_{x}(h)_{x}=0$ because the vector fields induced by $\mathcal{P}_{x}$ vanish at $x$; now $Q(h)_{x}=0$. If $Q$ generates $\mathrm{G}$, then $\mathrm{g}(h)_{x}=0$, so $\mathrm{G}_{0}(h)_{x}=0$ and we contradict nonconstancy of $h$ as above. Thus $Q=\Re^{e}+\sum_{\Delta}^{+} g_{\varphi}$ does not generate $g$. As $G_{0}$ is simple it follows that $G_{0} / K$ is hermitian symmetric, $Q$ is a parabolic subalgebra of $\mathcal{G}$ containing $P_{x}$, and (Borel embedding) $g K \rightarrow g Q$ embeds $G_{0} / K$ as an open $G_{0}$-orbit on the complex flag mainfold $Z=G / Q$. Let $\pi: X \rightarrow Z$ be the projection, $\pi\left(g P_{x}\right)=g Q$. Then $\pi$ is holomorphic and its restriction to $G_{0}(x)$ is the projection $g(x) \rightarrow g K$ of $G_{0}(x)$ to $G_{0} / K$. Note that $h$ is the lift of a function on $G_{0} / K$ because it must be constant on the compact complex submanifolds $g K(x), g \in G_{0}$, of $G_{0}(x)$. 
Now we consider the general case, using the notation preceding Definition 5.6. Let $h$ be a holomorphic function on $G_{0}(x)$. Let $1 \leqq i \leqq t$; fix $z_{s} \in G_{0}^{(s)}\left(x_{s}\right)$ for $s \neq i$, so $h\left(z_{1}, \cdots, z_{t}\right)=h_{i}\left(z_{i}\right)$ is a holomorphic function on $G_{0}^{(i)}\left(x_{i}\right)$. We have just proved that $h_{i}$ is constant if the conditions (5.5) do not all hold, and that $h_{i}$ is the lift of a function on $G_{0}^{(i)} / K^{(i)}$ if the conditions (5.5) do all hold. This proves that $h=f \cdot \psi$ where $\psi: G_{0}(x) \rightarrow D\left(G_{0}, x\right)$ is the natural projection and $f$ is a function $D\left(G_{0}, x\right)$. If $f$ is not holomorphic, $\bar{\partial} f \neq 0$, so $\bar{\partial} h=\psi * \bar{\partial} f \neq 0$ and $h$ is not holomorphic; thus $h=f \cdot \psi$ where $f$ is a holomorphic function on $D\left(G_{0}, x\right)$. Conversely, if $f$ is a holomorphic function on $D\left(G_{0}, x\right)$, then its lift $f \cdot \psi$ is holomorphic because $\psi$ is holomorphic. Q.E.D.

Two immediate consequences are:

5.8. Corollary. Let $X=G / P$ be a complex flag manifold, $G_{0}$ a real form of $G$, and $x \in X$ such that $G_{0}(x)$ is open in $X$.

1. The holomorphic functions separate points on $G_{0}(x)$, if and only if $\left(P_{x} \cap \tau P_{x}\right)_{0}$ is a maximal compact subgroup of $G_{0}$ and $G_{0} /\left(P_{x} \cap \tau P_{x}\right)_{0}$ is a hermitian symmetric coset space.

2. The following conditions are equivalent:

(2a) $G_{0}(x)$ carries a nonconstant holomorphic function.

(2b) $\operatorname{dim} D\left(G_{0}, x\right)>0$.

(2c) Let $\mathcal{H}_{0} \subset \mathcal{G}_{0} \cap \odot_{x}$ be a maximally compact Cartan subalgebra of $\mathcal{S}_{0}$. Then $\mathrm{G}_{0}$ has a simple ideal $G_{0}^{\prime}$, and $\mathcal{G}_{0}^{\prime}$ has a maximal compactly embedded subalgebra $\mathrm{K}^{\prime}$, such that

(i) $\mathfrak{H}_{0}^{\prime}=\mathfrak{H}_{0} \cap \mathcal{K}^{\prime}$ is a Cartan subalgebra of $\mathcal{G}_{0}^{\prime}$,

(ii) $\mathcal{G}_{0}^{\prime}{ }^{e} \cap \mathcal{P}_{x}=\mathcal{P}_{x}^{\prime}$ is given as $\mathcal{P}_{\Phi^{\prime}}^{\prime}$ where $\Phi^{\prime} \cup\left\{\varphi_{1}, \cdots, \varphi_{s}\right\}$ is a system of simple $\mathfrak{H C}_{0}^{\prime}{ }^{\mathrm{e}}$-roots of $\mathcal{G}_{0}^{\prime}{ }^{\mathrm{e}}$, and

(iii) $\mathfrak{K}^{\prime e}=\mathcal{P}_{\Psi^{\prime}}^{r}$ where $\Psi^{\prime}=\Phi^{\prime} \cup\left\{\varphi_{1}, \cdots, \varphi_{8-1}\right\}$ and $\varphi_{8}$ has coefficient 1 in the highest root.

Now we have seen that an extremely special situation is required in order that an open orbit $G_{0}(x) \subset X$ carry a nonconstant holomorphic function. In particular we cannot expect the closed $G_{0}$-orbit to be the Bergman-Silov boundary of an open orbit except in the usual [2] hermitian symmetric case.

6. Open orbits : invariant measure. In this section we develop the notion of measurable orbit for the case of an open orbit.

6.1. Definition. Let $X=G / P$ be a complex flag manifold, $x \in X$, and $G_{0}$ a real form of $G$. Then we say that $G_{0}(x)$ is a measurable open orbit if it is open in $X$ and carries $a G_{0}$-invariant volume element.

The elementary tool required is 
6.2. Lемма. Let $H_{0} \subset L \subset G_{0}$ be connected Lie groups where $G_{0}$ is reductive and $\mathrm{HC}_{0}$ is a Cartan subalgebra of $\mathcal{G}_{0}$. Then $G_{0} / L$ carries an invariant volume element if and only if $L$ is reductive.

Proof. $\mathscr{L}^{\mathfrak{e}}=\mathfrak{H}+\sum_{\Delta} \mathcal{G}_{\varphi}$ and $\mathcal{G}=\mathfrak{H}+\sum_{\Delta} \mathcal{S}_{\varphi}$ where $\mathfrak{H}=\mathfrak{H C}_{0}^{\mathfrak{e}}, \mathcal{G}=\mathcal{S}_{0}^{e}$ and the root systems $\Lambda \subset \Delta$. The representation $\chi$ of $L$ on the tangent space of $G_{0} / L$ complexifies to the restriction of $\operatorname{ad}_{G}(L)$ to $\sum_{\Delta-\Delta} S_{\varphi}$. Now $G_{0} / L$ has an invariant volume element if and only if $\operatorname{det} \chi=1$, and det $\chi=1$ if and only if ads(\&) acts on $\sum_{\Delta-\Delta} \oint_{\varphi}$ with trace 0 . As $\operatorname{ad}(\mathscr{L})$ acts on $\mathcal{S}$ with trace 0 , because $\mathcal{S}$ is reductive, now $G_{0} / L$ carries a $G_{0}$-invariant volume element if and only if $\operatorname{ad} \&(\mathscr{L})$ has trace 0 , i.e. if and only if $\sum_{\Lambda} \varphi=0$, i.e. if and only if $\varphi \in \Lambda$ implies $-\varphi \in \Lambda$, i.e. if and only if $L$ is reductive. Q.E.D.

Now we have a quick characterization

6.3. Theorem. Let $X=G / P$ be a complex flag manifold, $x \in X$, and $G_{0}$ a real form of $G$. Then the following conditions are equivalent, and each implies that $G_{0}(x)$ is open in $X$.

(1a) $G_{0}(x)$ is a measurable open orbit.

(1b) $G_{0}(x)$ has a $G_{0}$-invariant volume element.

(1c) $G_{0}(x)$ has a $G_{0}$-invariant, possibly indefinite, kaehler metric.

(1d) The isotropy subgroup $G_{0} \cap P_{x}$ is the centralizer in $G_{0}$ of $a$ (compact) toral subgroup $T \subset G_{0}$.

(2a) $\odot_{x} \cap \tau P_{x}$ is reductive, i.e. $\odot_{x} \cap \tau \odot_{x}=\odot_{x}^{r} \cap \tau P_{x}^{r}$.

(2b) $P_{x} \cap \tau P_{x}=P_{x}^{r}$.

(2c) $\tau \mathcal{P}_{x}^{u}=\mathcal{P}_{x}^{-u}$, i.e. $\mathcal{S}=\mathcal{P}_{x}+\tau \mathcal{P}_{x}^{u}$.

(2d) $\odot_{x}=\odot_{\Phi}$ with $\tau \Phi^{r}=\Phi^{r}$ and $\tau \Phi^{u}=-\Phi^{u}$.

Proof. Let $\mathcal{H C} \subset \boldsymbol{P}_{x}$ be a $\tau$-stable Cartan subalgebra of $\boldsymbol{g}$ and let $\boldsymbol{I I}$ be a simple root system such that $P_{x}=\rho_{\Phi}$ for a subset $\Phi \subset$ II. Lemma 2.10 says $\left(\mathcal{P}_{x} \cap \tau \mathcal{P}_{x}\right)^{r}=\mathcal{P}_{x}^{r} \cap \tau \mathcal{P}_{x}^{r}$, root system $\Phi^{r} \cap \tau \Phi^{r}$, and $\left(\mathcal{P}_{x} \cap \tau \mathcal{P}_{x}\right)^{u}$ $=\left(\mathcal{P}_{x}^{r} \cap \tau \mathcal{P}_{x}^{u}\right)+\left(\mathcal{P}_{x}^{u} \cap \tau \mathcal{P}_{x}^{r}\right)+\left(\mathcal{P}_{x}^{u} \cap \tau \mathcal{P}_{x}^{u}\right)$, root system $\left(\Phi^{r} \cap \tau \Phi^{u}\right)$ $\cup\left(\Phi^{u} \cap \tau \Phi^{r}\right) \cup\left(\Phi^{u} \cap \tau \Phi^{u}\right)$.

(2d) implies (2b) by summing root spaces and (2b) visibly implies (2a). If $\mathcal{P}_{x} \cap \tau \odot_{x}$ is reductive then $\tau \Phi^{u} \cap\left(\Phi^{r} \cup \Phi^{u}\right)$ is empty, so $\tau \Phi^{u}$ $=-\Phi^{u}$; thus (2a) implies (2c). If $\tau \Phi^{u}=-\Phi^{u}$ then $\tau$ preserves $\left\{\varphi \in \Delta: \varphi \notin \Phi^{u} \cup-\Phi^{u}\right\}$, which is $\Phi^{r}$; thus (2c) implies (2d). Now (2a), (2b), (2c) and (2d) are equivalent and each implies that $\Phi^{u} \cap \tau^{u} \Phi^{u}$ is empty, i.e. that $G_{0}(x)$ is open in $X$. It follows from Lemma 6.2 that (1a), (1b), (2a), (2b), (2c) and (2d) are equivalent.

Let $G_{0}(x)$ be a measurable open orbit, i.e. $\tau \Phi^{r}=\Phi^{r}$ and $\tau \Phi^{u}=-\Phi^{u}$. $\tau$ preserves $\mathcal{P}_{x}^{r}$, hence its center $\mathcal{H}_{\Phi}=\{h \in \mathcal{H}: \Phi(h)=0\}$; thus $\mathcal{G}_{0} \cap \mathcal{H}_{\Phi}$ $=\mathcal{H}_{T}^{\prime}+\mathfrak{H}_{V}^{\prime}$ sum of toral and vector parts. We define 


$$
A=\left\{\varphi \in \Phi^{u}: \varphi\left(\mathcal{F C}_{T}^{\prime}\right)=0\right\} \quad \text { and } \quad \alpha=\sum_{\Phi^{u}} \varphi .
$$

$\boldsymbol{\tau} \Phi^{u}=-\Phi^{u}$ says $\tau(\alpha)=-\alpha$, so $\alpha \in i \mathcal{H C}_{T}$. If $\varphi \in \Phi^{u}$ then $\varphi=\varphi_{1}+\varphi_{2}$ with $\varphi_{1}\left(\mathcal{H}_{\Phi}\right)=0$ and $\varphi_{2} \in i \mathcal{H C}_{T}^{\prime}+\mathcal{H}_{V}^{\prime}$. Projection to the trivial representation space of the Weyl group of $\mathcal{P}_{x}^{r}$ on $i \mathcal{F}_{T}+\mathcal{F}_{V}$ annihilates $\varphi_{1}$, and that Weyl groups preserves $\Phi^{u}$, so $\alpha \in i \mathcal{F}_{T}^{\prime}+\mathcal{H}_{V}^{\prime}$. Now $\alpha \in i \mathcal{H}_{T}^{\prime}$. If $\varphi \in \Phi^{u}$ then $\langle\varphi, \alpha\rangle>0$. Thus $A$ is empty. Now $\left\{\varphi \in \Delta: \varphi\left(\mathcal{F}_{T}^{\prime}\right)=0\right\}$, which contains $\Phi^{r}$, contains no element of $\Phi^{u} \cup-\Phi^{u}$. In other words $P_{x}^{r}$ is the centralizer of $\mathcal{F C}_{T}^{\prime}$ in $\mathcal{G}$, so $G_{0} \cap P_{x}=\left(P_{x}^{r}\right)_{0}$ is the centralizer in $G_{0}$ of the (compact) torus $T=\exp \left(\mathcal{H C}_{T}^{\prime}\right)$. We have proved that (1a) implies (1d).

Let $G_{0} \cap P_{x}$ be the centralizer of a torus $T \subset G_{0}$. Choose $t \in J$ such that $\mathcal{G}_{0} \cap \rho_{x}$ is the centralizer of $t$ in $\mathcal{S}_{0}$, and let $\eta$ denote the left invariant 1 -form on $G_{0}$ whose value at the identity is given by $\eta(h)=\langle t, h\rangle$. Then $\bar{\omega}=d \eta$ is a left invariant 2 -form on $G_{0}$, right invariant by $G_{0} \cap P_{x}$, inducing a nondegenerate $G_{0}$-invariant 2 -form $\omega$ on $G_{0}(x)$ $=G_{0} /\left(G_{0} \cap P_{x}\right)$. Now the invariant almost complex structure $J$ on $G_{0}(x)$, together with $\omega$, defines an invariant hermitian metric with kaehler form $\omega$. As $d \bar{\omega}=d(d \eta)=0$ is the lift of $d \omega$ to $G_{0}$, it follows that $d \omega=0$ and the metric is kaehler. Thus (1d) implies (1c). And obviously (1c) implies (1b) because a kaehler metric (of any signature) has a volume element. Q.E.D.

6.4. Corollary. Let $X=G / P$ be a complex flag manifold, $G_{0}$ a real form of $G$, and $x \in X$ such that $G_{0}(x)$ is open in $X$. If $G_{0}$ has its maximal compact subgroups $K$ such that rank $K=\operatorname{rank} G_{0}$, then $G_{0}(x)$ is a measurable open orbit.

Proof. Let $\mathcal{H C} \subset P_{x}$ be a $\tau$-stable Cartan subalgebra of $\mathcal{G}$, such that $\mathfrak{H}_{0}=\mathfrak{H} \cap \mathcal{G}_{0}$ is a maximally compact Cartan subalgebra of $\mathcal{G}_{0}$. If rank $K=\operatorname{rank} G_{0}$, then $\mathcal{H}_{0}$ is compact, so $\tau \varphi=-\varphi$ for every $\mathcal{H}$-root $\varphi$; then $\tau \Phi^{r}=\Phi^{r}$ and $\tau \Phi^{u}=-\Phi^{u}$ where $\mathcal{P}_{x}=\mathcal{P}_{\Phi}$. Q.E.D.

The condition rank $K=\operatorname{rank} G_{0}$ is not necessary for an open orbit to be measurable. For example

6.5. Corollary. Let $X=G / B$ be a complex flag manifold where $B$ is a Borel subgroup of $G$. Let $G_{0}$ be a real form of $G$. Then every open $G_{0}$-orbit on $X$ is measurable.

Proof. $B=\mathcal{P}_{\Phi}$ where $\Phi$ is empty. Thus $\tau \Phi^{r}=\Phi^{r}$, empty set, and $\tau \Phi^{u}=\tau \Delta^{+}=\Delta^{-}=-\Phi^{u}$. Q.E.D.

The main point of Corollaries 6.4 and 6.5 is that measurability does not depend on the choice of open orbit. In order to prove that in general we need the notion of opposed parabolic subgroups. 
6.6. Definitions. The opposition automorphism of a reductive complex Lie algebra $\mathrm{g}$ relative to a Cartan subalgebra $\mathfrak{H}$ is the automorphism

$$
\nu: \mathcal{G} \rightarrow \mathcal{G} \text { by } \quad \nu(h)=-h \quad \text { for } h \subset \mathfrak{H} \quad \text { and } \quad \nu\left(e_{\varphi}\right)=e_{-\varphi}
$$

where $\left\{h_{*} ; e_{\varphi}\right\}$ is a Weyl basis of $\mathcal{G}$. Note that any two opposition automorphisms are conjugate by an inner automorphism. Two subsets $\delta$, $\mathcal{S}^{\prime} \subset \mathcal{S}$ are opposed if $S$ is conjugate to $\nu\left(\mathcal{S}^{\prime}\right)$ by an inner automorphism, for some (hence any) opposition automorphism of $g$.

Let $\beta$ be a parabolic subalgebra of a reductive or semisimple complex Lie algebra $\mathcal{G}$. Choose a Cartan subalgebra $\mathcal{H} \subset \mathcal{P}$ of $\mathcal{G}$ and a simple root system $\Pi$ such that $\beta=\rho_{\Phi}$ for a subset $\Phi \subset \Pi$. Let $\nu$ be the opposition automorphism for this data and let $w$ be the Weyl group element such that $w(\nu \Pi)=\Pi$. Then of course the parabolic $\nu(P)$ opposed to $P$ is conjugate to $P_{w \nu \Phi}$. We can see the action of $w \nu$ on $\Pi$ via the Dynkin diagram. It preserves each component, for $\nu$ preserves each simple ideal of $\mathcal{G}$; if a simple ideal has $-I$ in the Weyl group, i.e. if it is not of type $A_{l}(l>1), D_{2 n+1}(n>0)$ or $E_{6}$, then $w=\nu=\nu^{-1}$ on that ideal, so $w \nu$ acts trivially on the corresponding component of the Dynkin diagram; if a simple ideal does not have $-I$ in the Weyl group, then $w \nu$ acts on its Dynkin diagram by:

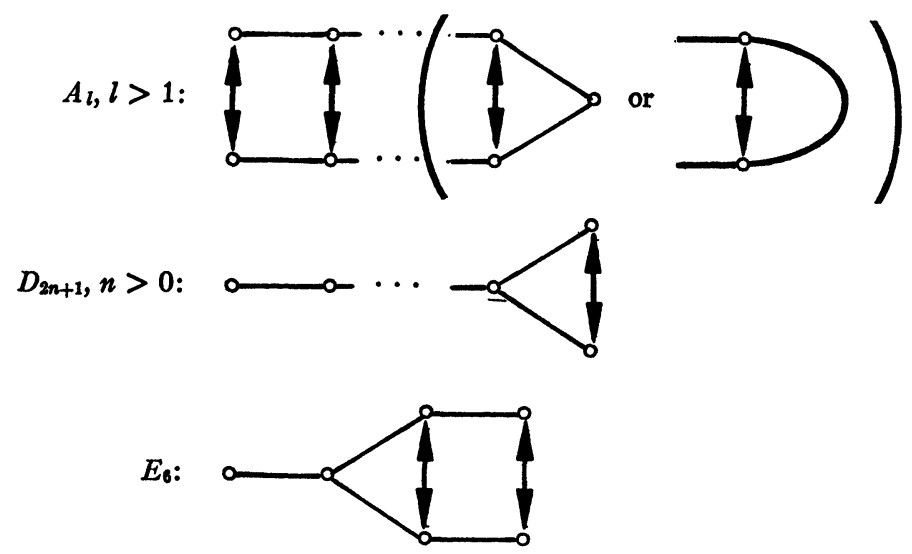

6.7. Theorem. Let $X=G / P$ be a complex flag manifold and $G_{0} a$ real form of $G$. Then the following conditions are equivalent:

(i) $\odot$ and $\tau \odot$ are opposed parabolic subalgebras of $\mathcal{G}$.

(ii) Some open $G_{0}$-orbit on $X$ is measurable.

(iii) Every open $G_{0}$-orbit on $X$ is measurable. 
Proof. (iii) implies (ii) because $G_{0}$ has an open orbit.

Assume (ii) and let $G_{0}(x)$ be a measurable open orbit. $\mathfrak{H C} \subset \mathcal{P}_{x}$ is a $\tau$-stable Cartan subalgebra of $\mathcal{G}$ such that $\mathcal{H}_{0}=\mathfrak{H} \cap \mathcal{G}_{0}$ is a maximally compact Cartan subalgebra of $\mathcal{S}_{0}$. Now $\rho_{x}=\rho_{\Phi}$ where $\Phi$ is contained in a simple root system $\Pi$. We take the opposition automorphism $\nu$ for $\mathcal{H}$ and $\Pi$. Using Theorem 6.3, $\tau \Phi^{u}=-\Phi^{u}=\nu \Phi^{u}$, so $\tau \odot_{x}$ and $\nu \odot_{x}$ each is the normalizer of $P_{x}^{-u}$ in $\mathcal{G}$, whence $\tau P_{x}=\nu P_{x}$. Thus $\tau \odot$ is conjugate to $\nu \odot$, i.e. $\odot$ is opposed to $\tau \odot$. That shows that (ii) implies (i).

Let $\odot$ and $\tau \odot$ be opposed, let $G_{0}(x)$ be an open orbit, and let $\mathcal{H}_{0} \subset \mathcal{S}_{0}$ $\cap \rho_{x}$ be a maximally compact Cartan subalgebra of go $_{0}$. Now $\boldsymbol{\rho}_{x}=\boldsymbol{\rho}_{\Phi}$ where $\Phi$ is contained in a simple root system $I$ such that $\tau(I I)=-\Pi$. $\tau \mathcal{P}_{x}$ and $\nu \mathcal{P}_{x}$ are conjugate parabolic subalgebras of $\mathcal{G}$ with a common Borel subalgebra $\mathcal{F}_{0}^{e}+\sum_{\Delta}+\mathcal{S}_{-\varphi}$; thus $\tau \odot_{x}=\nu \mathcal{P}_{x}$. Now $\boldsymbol{P}_{x} \cap \tau \mathcal{P}_{x}=\mathcal{P}_{x}$ $\cap \nu P_{x}=P_{x}^{r}$, so $G_{0}(x)$ is measurable by Theorem 6.3. Thus (i) implies (iii). Q.E.D.

We recover Corollary 6.4 from Theorem 6.7 because $\tau \varphi=-\varphi=\nu \varphi$ relative to a compact Cartan subalgebra $\mathcal{H}_{0} \subset \mathrm{Cg}_{0}$, and we recover Corollary 6.5 because $B, \tau \circledast$ and $\nu \circledast$ are all conjugate. But, in addition, we have

6.8. Corollary. Let $X=G / P$ be a complex flag manifold. Let $G_{0}$ be a real form of $G$ such that $\mathcal{G}_{0}$ is a direct sum of absolutely simple ideals $S_{0}^{(t)}$ each of which has one or more of the properties

(i) $\mathrm{S}_{0}^{(i)}$ is not of type $A_{l}(l>1), D_{2 n+1}(n>0)$ nor $E_{6}$; or

(ii) the maximal compactly embedded subalgebras of $\mathrm{g}_{0}^{(i)}$ have maximal rank; or

(iii) $P \cap \mathrm{g}^{(i)}$ is a Borel subalgebra of $\mathrm{g}^{(i)}=\mathrm{G}_{0}^{(i) e}$.

Then every open $G_{0}$-orbit on $X$ is measurable.

The key proviso of Corollary 6.8 is that the simple ideals of $g_{0}$ are absolutely simple, i.e. that $\tau$ preserves every simple ideal of $\mathcal{G}$. But it is interesting to consider the other extreme.

Let $G_{0}$ be a complex semisimple connected Lie group viewed as a real Lie group. Then $G=G_{0}^{\mathfrak{e}}$ is $G_{0} \times G_{0}$ and the embedding $G_{0} \subset G$ is given by $g \rightarrow \delta(g)=(g, g) . \tau$ acts on $G$ by $\tau\left(g_{1}, g_{2}\right)=\left(g_{2}, g_{1}\right) . P=P_{1} \times P_{2}$ where $P_{i}$ are parabolic subgroups of $G_{0}$. In particular $\tau(P)=P_{2} \times P_{1}$ where $P=P_{1} \times P_{2}$. The opposition automorphism acts by $\nu(P)=\nu\left(P_{1}\right)$ $\times \nu\left(P_{2}\right)$ where $P=P_{1} \times P_{2}$. Thus $\beta$ and $\tau \odot$ are opposed if and only if $\rho_{1}$ and $P_{2}$ are opposed. In other words, the open $G_{0}$-orbits on $G / P$ are measurable if and only if $P=P_{1} \times P_{2}, \odot_{1}$ and $P_{2}$ opposed, i.e. if and only if $P=P_{1} \times \nu P_{1}$. If $G_{0}$ has rank $r$ as a complex group, then $G$ has rank $2 r$, so $G$ has $2^{2 r}$ conjugacy classes of parabolic subgroups $P=P_{1} \times P_{2}$ and just $2^{r}$ of them are of the form $P_{1} \times \nu P_{1}$. Thus "most" parabolic sub- 
groups $P \subset G$ have the property that the open $G_{0}$-orbits on $G / P$ are not measurable.

7. Integrable orbits. Let $X=G / P$ be a complex flag manifold, $x \in X$ and $G_{0}$ a real form of $G$. We observe $\rho_{x}+\tau \odot_{x}=\rho \Leftrightarrow \rho_{x}^{-u} \subset \tau \rho^{\prime}$ $\Leftrightarrow \mathcal{P}_{x}^{-u} \cap \tau \odot_{x}^{-u}=0 \Leftrightarrow \odot_{x}^{u} \cap \tau{\odot_{x}^{u}}_{x}=0 \Leftrightarrow G_{0}(x)$ is open in $X$. This leads us to some definitions whose mystery will evaporate in $\$ 9$.

7.1. Definitions. Let $X=G / P$ be a complex flag manifold, $x \in X$ and $G_{0}$ a real form of $G$. If $P_{x}+\tau \odot_{x}$ is a subalgebra of $\mathcal{G}$, then we say that

$\mathcal{P}_{x}$ is an integrable parabolic subalgebra of $\mathrm{G}$,

$P_{x}$ is an integrable parabolic subgroup of $G$,

$x$ is an integrable point of $X$, and

$G_{0}(x)$ is an integrable $G_{0}$-orbit on $X$.

These notions are, of course, dependent on choice of the real form $G_{0}$.

The basic facts are given by

7.2. TheOREM. Let $\mathrm{g}$ be a complex semisimple Lie algebra, $\odot$ a parabolic subalgebra, and $\mathrm{g}_{0}$ a real form of $\mathrm{G}$. Denote

$$
\mathcal{u}=\mathcal{P}^{u} \cap \tau \mathcal{P}^{u} \text { and } \mathfrak{x} \text { is the normalizer of } \mathcal{U} \text { in } \mathrm{g} .
$$

Then the following conditions are equivalent:

(i) $\odot$ is integrable, i.e. $\odot+\tau \odot$ is a subalgebra of $\mathrm{G}$.

(ii) $\rho+\tau \odot=\Re$.

(iii) $\mathcal{P}+\tau \odot$ is an algebra and $\mathcal{U}$ is its nilpotent radical.

(iv) $\mathcal{U}$ is the nilpotent radical of $\Re$.

(v) $\odot$ normalizes $\mathcal{U}$, i.e. $\odot \subset \Re$.

Proof. Relative to a $\tau$-stable Cartan subalgebra $\mathfrak{H} \subset \mathcal{P}$ of $\mathcal{Q}$ and a subset $\Phi$ of a simple root system such that $\mathcal{P}=\mathcal{P}_{\Phi}$, we have $\mathcal{P}+\tau \mathcal{P}$ $=\mathfrak{F}+\sum_{\Gamma} \oint_{\varphi}$ where $\Gamma=\Phi^{r} \cup \tau \Phi^{r} \cup \Phi^{u} \cup \tau \Phi^{u}$. If $\varphi \in \Gamma$ and $-\varphi \notin \Gamma$ it follows that $\varphi \in \Phi^{u} \cup \tau \Phi^{u}, \varphi \notin \Phi^{u} \cap \tau \Phi^{r}$ and $\varphi \notin \Phi^{r} \cap \tau \Phi^{u}$; then $\varphi \in \Phi^{u}$ $\cap \tau \Phi^{u}$ or $\varphi \in \Phi^{u} \cap-\tau \Phi^{u}$ or $\varphi \in-\Phi^{u} \cap \tau \Phi^{u}$; if $\varphi>0$ and $\tau \varphi>0$ we conclude that $\varphi \in \Phi^{u} \cap \tau \Phi^{u}$.

Let $\mathcal{P}+\tau \odot$ be an algebra. The discussion above shows that it has nilpotent radical $u$. Thus (i) implies (iii). A parabolic subalgebra is the normalizer of its nilpotent radical; thus (iii) implies (ii). And visibly (ii) implies (i). Now (i), (ii) and (iii) are equivalent.

If $\mathcal{P}+\tau \mathcal{P}=\Re$ then $\mathcal{P} \subset \Re$; thus (ii) implies (v). If $P \subset \Re$ then $\Re$ is parabolic; $\tau \mathscr{U}=\mathcal{U}$ implies $\tau \mathscr{N}=\mathfrak{\Re}$ so $\odot+\tau \odot \subset \Re$; now the discussion of the first paragraph of the proof shows that $u$ contains the nilpotent radical of $\Re$; thus ( $v$ ) implies (iv). Now we need only prove that (iv) implies (ii).

Let $\mathcal{U}$ be the nilpotent radical of $\Re$. If $\Re$ is a parabolic subalgebra of $\mathcal{G}$, then $\mathfrak{l}=\mathcal{P}_{\Psi}$ with $\Phi \subset \Psi$ such that $\Psi^{u}=\Phi^{u} \cap \tau \Phi^{u}$. As $\Delta^{+}$is the dis- 
joint union of $\Phi^{r} \cap \Delta^{+}, \Phi^{u} \cap\left(\tau \Phi^{r} \cup-\tau^{u}\right)$ and $\Phi^{u} \cap \tau \Phi^{u}$, and also is the disjoint union of $\Psi^{r} \cap \Delta^{+}$and $\Psi^{u}=\Phi^{u} \cap \tau \Phi^{u}$, it follows that $\Psi^{r} \cap \Delta^{+}$ $=\left(\Phi^{r} \cap \Delta^{+}\right) \cup\left(\Phi^{u} \cap \tau \Phi^{r}\right) \cup\left(\Phi^{u} \cap-\tau \Phi^{u}\right)$ so $\Re=\rho^{r}+\rho^{u}+\tau \rho^{r}+\tau \boldsymbol{\rho}^{u}=\rho$ $+\tau \odot$. Thus (iv) implies (ii) provided that (iv) forces $\Re$ to be parabolic. In other words, the theorem is reduced to

7.3. LеммA. Let $\mathcal{g}$ be a complex semisimple Lie algebra, let $u$ be a nilpotent ${ }^{5}$ subalgebra, and let $\mathfrak{x}$ be the normalizer of $\mathfrak{U}$ in $\mathcal{G}$. Suppose that $\mathfrak{T}$ contains a Cartan subalgebra $\mathfrak{H}$ of $\mathcal{G}$ and that $\mathcal{U}$ is the nilpotent ${ }^{5}$ radical of $\mathfrak{N}$. Then $\mathfrak{N}$ is a parabolic subalgebra of $\mathcal{G}$.

Proof. $\mathfrak{K}=\mathfrak{L}+\mathfrak{U}$ semidirect where $\mathfrak{H C} \mathfrak{L}$ and $\mathfrak{L}$ is reductive. $\mathcal{U}=\sum_{A} \mathcal{S}_{\varphi}$ for some set $A$ of $\mathcal{H}$-roots of $\mathcal{G}$. $\mathfrak{L}=\mathfrak{L}^{\prime} \oplus \mathcal{S}$ where $\mathcal{S} \subset \mathcal{H C}$ is the center of $\mathscr{L}$ and $\mathscr{L}^{\prime}=[\mathscr{L}, \mathscr{L}]$ is the semisimple part. $\mathfrak{H}+\mathcal{U}$ is contained in a Borel subalgebra of $\mathcal{G}$, so we order the roots such that $\varphi>0$ for every $\varphi \in A$.

Let $\mathcal{L}$ be semisimple, let $W_{L}$ be its Weyl group and define $\alpha=\sum_{A} \varphi$. Then $\alpha$ is a $W_{L}$-invariant positive sum of positive roots. The action of $W_{L}$ on $\mathcal{H}$ has no trivial subrepresentation. Thus $\alpha=0$ and $A$ is empty. It follows that $\mathfrak{u}=0$, so $\Re=g$, which is a parabolic subalgebra of $\mathcal{G}$.

Now we may assume $\mathscr{L}$ not semisimple, so its center $\delta \neq 0$. Define $B=\{\varphi \in A: \varphi(\delta)=0\}$ and $\beta=\sum_{B} \varphi$. As above it follows that $\beta=0$ so $B$ is empty. Let $\mathcal{Z}$ be the centralizer of $\mathcal{S}$ in $\mathcal{G}$ and order the weights of $\mathcal{S}$ so that positive weight spaces are sums of positive root spaces. Then the corresponding parabolic subalgebra $\mathcal{Q}$ of $\mathcal{G}$ has $Z=Q^{r}$, and $u \subset Q^{u}$ because $\mathfrak{Z} \cap u=0$. Let $w=\mathfrak{N} \cap Q^{u}$; so $u \subset W \subset Q^{u}$. If $u \neq Q^{u}$ then $u \neq w$ because a proper nilpotent subalgebra of a nilpotent Lie algebra cannot be its own normalizer. But $\mathfrak{\tau}=\mathfrak{L}+\mathcal{U}$ with $\mathcal{L} \subset \mathcal{Q}^{r}$ and $\mathcal{U}$ is the nilpotent radical of $\mathfrak{T}$; thus $\mathcal{U}=\mathfrak{N} \cap \mathcal{Q}^{u}=\mathscr{W}$; now $\mathcal{U}=\mathcal{Q}^{u}$ so $\Re=Q$. Q.E.D.

Now we go on to construct all integrable $G_{0}$-orbits on $X=G / P$.

Let $\mathcal{G}$ be a complex semisimple Lie algebra, $\mathfrak{H C}$ a Cartan subalgebra and $\Pi$ a simple root system. We know that each conjugacy class of parabolic subalgebras of $\mathcal{G}$ is represented by just one algebra $P_{\Phi}$, $\Phi \subset I I$; in particular, if $\odot$ and $Q$ are parabolic subalgebras of $\mathcal{G}$, then the conditions

(i) $Q$ contains a conjugate of $P$,

(ii) $P$ is contained in a conjugate of $Q$, and

(iii) $\mathcal{P}$ is conjugate to $\mathcal{P}_{\Phi}, Q$ to $\mathcal{P}_{\Psi}$, with $\Phi \subset \Psi \subset \Pi$, are equivalent.

5 This means that $\mathfrak{U}$ is represented by nilpotent matrices in the adjoint representation of $\mathcal{G}$. 
7.4. Definition. We write $\beta \prec Q$ to denote the equivalent conditions (i), (ii) and (iii) above.

Now let $\mathcal{S}_{0}$ be a real form of $\mathcal{G}$ and assume $\mathfrak{H}$ stable under the complex conjugation $\tau$ of $\mathcal{G}$ over $\mathcal{S}_{0}$. Let $w \in W_{G}$ be the Weyl group element which carries the simple root system $\tau$ (II) back to II. We have a (wr)-stable subset $\Psi_{0} \subset \Pi$ determined by: $(\Re+a+\Re)^{\mathfrak{e}}$ is conjugate to $\mathcal{P}_{\Psi_{0}}$ where $\mathfrak{N}+a+\mathfrak{N}$ is the minimal parabolic subalgebra of $g_{0}$. Now the conjugacy classes of parabolic subalgebras, such that the class contains an algebra defined/R (i.e. $\tau$-stable), are represented by the $\mathcal{P}_{\Psi}$ such that
(a) $(w \tau) \Psi=\Psi$ and
(b) $\Psi_{0} \subset \Psi \subset \Pi$.

In order to apply the criterion (7.5), one uses the fact [15, p. 909] that, if $\mathcal{G}$ is simple, then $w \tau$ acts trivially on II except in the cases:

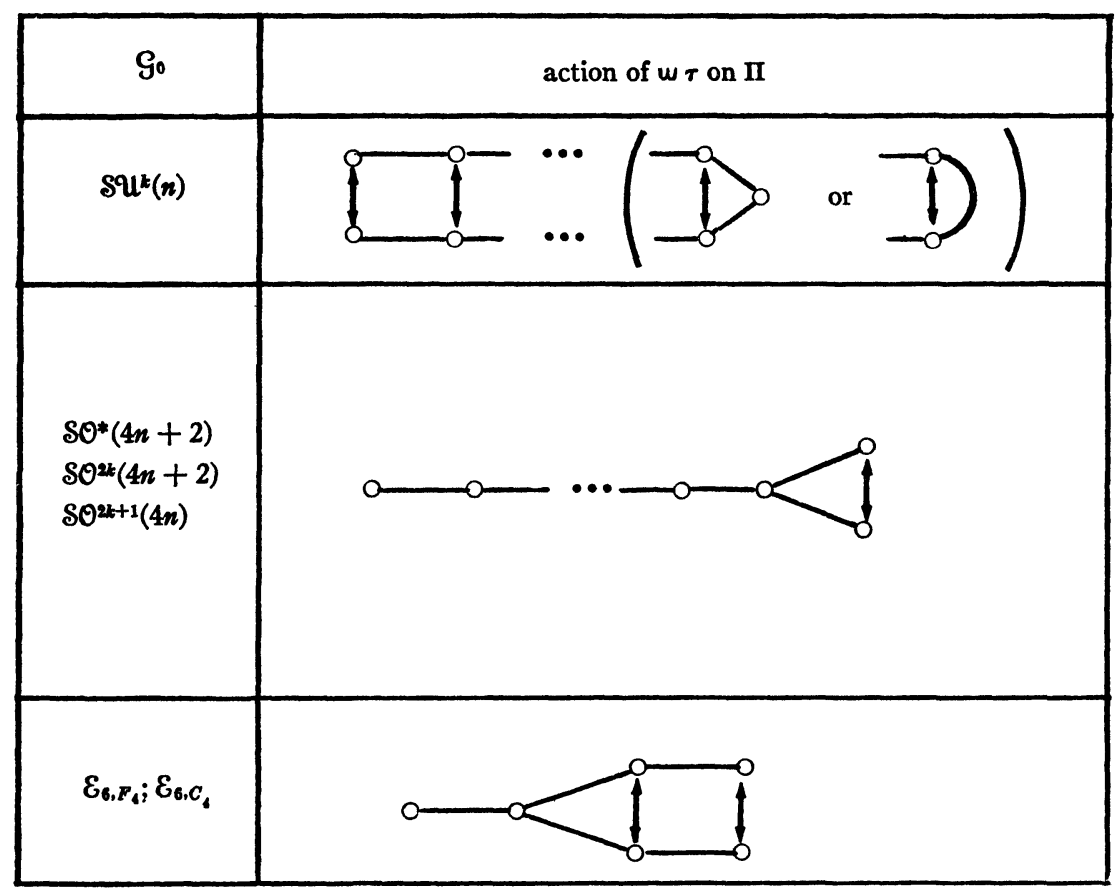

Now suppose that we have a fixed parabolic subalgebra $\odot \subset g$. We look for the $\tau$-stable parabolic subalgebras $Q \subset \mathcal{Q}$ such that $P \prec Q$. Given such an algebra $Q$ we have a conjugate $\rho_{x}$ of $\beta$ such that $\mathcal{P}_{x}+\tau \mathcal{P}_{x} \subset Q$. Suppose that $P$ is conjugate to $\mathcal{P}_{\Phi}$ and $Q$ is conjugate to $\mathcal{P}_{\Psi}$; then $\Phi \subset \Psi$ and $\Psi$ satisfies (7.5). In other words
(a) $(w \tau) \Psi=\Psi$ and
(b) $\left\{\Psi_{0} \cup \Phi \cup(w \tau) \Phi\right\} \subset \Psi \subset \Pi$. 
Conversely, if $\Psi$ satisfies (7.6), then $P_{\Psi}$ has a $\tau$-stable conjugate $Q$ because $\Psi$ satisfies (7.5), and $\rho \prec Q$ because $\Phi \subset \Psi$. This proves the first statement of

7.7. Theorem. Let $X=G / P$ be a complex flag manifold, $G_{0}$ a real form of $G$, HC a $\tau$-stable Cartan subalgebra of $\mathcal{G}, \Pi$ a simple $\mathfrak{H C}$-root system, and $\Phi \subset \Pi$ such that $\beta$ is conjugate to $\rho_{\Phi}$. Let $w \in W_{G}$ such that $(w \tau) \Pi=\Pi$ and define

$$
\Psi_{X}=\Psi_{0} \cup \Phi \cup(w \tau) \Phi
$$

1. The G-conjugacy classes of parabolic subalgebras $Q \subset \mathcal{G}$ such that

$$
P \prec Q \text { and } \tau(Q)=Q
$$

are the classes represented by algebras $\odot_{\Psi}$ such that

$$
(w \tau) \Psi=\Psi \text { and } \Psi_{X} \subset \Psi \subset \Pi \text {. }
$$

2. If $Q \subset \mathcal{G}$ is a $\tau$-stable parabolic subalgebra then the following conditions are equivalent:

(2a) $\$ \prec Q$.

(2b) $Q=\odot_{x}+\tau \odot_{x}$ for some (necessarily integrable) $x \in X$.

(2c) $Q$ is conjugate to $P_{\Psi}$ with $\Psi_{X} \subset \Psi \subset \Pi$.

Proof. Statement (1) was proved in the discussion preceding the theorem.

If $\tau(Q)=Q$ and $P \prec Q$ then (1) implies that $Q$ is conjugate to $P_{\Psi}$ with $\Psi_{X} \subset \Psi \subset \Pi$. If $\tau(Q)=Q$ and $Q$ is conjugate to $P_{\Psi}$ with $\Psi_{X} \subset \Psi \subset \Pi$ then $\Phi \subset \Psi$ so $\rho \prec Q$. Now (2a) and (2c) are equivalent. As (2b) trivially implies (2a), we now need only check that (2a) implies (2b).

Let $\tau(Q)=Q$ and $\beta \prec Q$. If we have a Borel subalgebra $B \subset Q$ of $g$ such that $B+\tau B=Q$, then we choose a conjugate $\rho_{x}$ of $P$ such that $B \subset P_{x} \subset Q$; that gives $Q=B+\tau B \subset P_{x}+\tau P_{x} \subset Q+\tau Q=Q$, and we conclude $P_{x}+\tau P_{x}=Q$. Now the proof of Theorem 7.7 comes down to the proof of

7.9 LEMMA. Let $\mathrm{g}$ be a complex semisimple Lie algebra, Go a real form of $\mathcal{G}$, and $Q$ a $\tau$-stable parabolic subalgebra of $\mathcal{G}$. Then there is a Borel subalgebra $B \subset Q$ of $\mathcal{G}$ with $B+\tau B=Q$.

Proof. $Q=Q^{r}+Q^{u}$ with each summand stable under $\tau$. Now $Q_{0}^{r}=S_{0}$ $\cap Q^{r}$ is a real form of $Q^{r}$ and is a reductive subalgebra of maximal rank in So $_{0}$. Let $\mathfrak{H C}_{0}=\mathfrak{H C}_{T}+\mathfrak{H C}_{V}$ be a maximally compact Cartan subalgebra of $Q_{0}^{r}$. Apply Theorem 4.5 to the action of $Q_{0}^{r}$ on $Q^{r} /($ Borel subgroup); it shows that $Q^{r}$ has a Borel subalgebra $B_{1}$ such that $B_{1}+\tau B_{1}=Q^{r}$. 
Now $B=B_{1}+Q^{u}$ is a Borel subalgebra of $\mathcal{G}$, and $B+\tau B=Q^{r}+Q^{u}=Q$.

We extend the proof that (2a) implies (2b) in Theorem 7.7 to an existence and enumeration theorem for integrable orbits.

7.10. Theorem. Let $X=G / P$ be a complex flag manifold, $G_{0}$ a real form of $G$, and $\mathcal{H C}$ a $\tau$-stable Cartan subalgebra of $\mathrm{G}$. Let $\Pi$ I be a simple HC-root system, $w \in W_{G}$ such that $(w \tau) \Pi=\Pi, \Phi \subset \Pi$ such that $P$ is conjugate to $\odot_{\Phi}$, and $\Psi_{X}=\Phi \cup(w \tau) \Phi \cup \Psi_{0}$, (wr)-stable subset of $\Pi$. For every set $\Psi$ such that $\Psi_{X} \subset \Psi \subset \Pi$ and $(w \tau) \Psi=\Psi$, choose a $\tau$-stable parabolic subalgebra $Q_{\Psi} \subset \mathcal{G}$ which is conjugate to $\odot_{\Psi}$, and choose a maximally compact Cartan subalgebra $\mathcal{H}_{\Psi, 0}$ of the real form $\mathcal{G}_{0} \cap Q_{\Psi}^{r}$ of $Q_{\Psi}^{r}$.

1. If $B_{1}$ is a Borel subalgebra of $Q_{\Psi}^{r}$ which contains $\mathcal{H C}_{\Psi}=\mathcal{F C}_{\Psi, 0}^{e}$ and such that $B_{1}+\tau B_{1}=Q_{\Psi}^{r}$, and if $B$ denotes the Borel subalgebra $B_{1}+Q_{\Psi}^{u}$ of $\mathcal{S}$, and if $P_{x}$ is the conjugate of $P$ such that $B \subset P_{x} \subset Q_{\Psi}$, then

(1a) $x$ is an integrable point of $X$,

(1b) $G_{0}(x)$ is an integrable $G_{0}$-orbit on $X$, and

(1c) $\rho_{x}+\tau P_{x}=Q_{\Psi}$.

2. If $\mathrm{z} \in X$ is an integrable point, and if the parabolic subalgebra $\mathcal{P}_{z}+\tau \odot_{z}$ is conjugate to $\mathcal{P}_{\Psi}$, then in (1) we can choose the Borel subalgebra $\bigotimes_{1}$ of $Q_{\Psi}^{r}$ in such a manner that $z \in G_{0}(x)$.

3. For each set $\Psi, \Psi_{X} \subset \Psi \subset \Pi$ and $(w \tau) \Psi=\Psi$, choose one point $x_{\Psi} \in X$ as constructed in (1). Let $Q_{\Psi, 0}^{r}$ denote the identity component of $G_{0} \cap Q_{\Psi}^{r}$. Then $Q_{\Psi}\left(x_{\Psi}\right)=Q_{\Psi}^{r}\left(x_{\Psi}\right)=Q_{\Psi}^{r} /\left(Q_{\Psi}^{\tau} \cap P_{x \Psi}\right)$ is a complex flag manifold $X_{\Psi}$, and the open $Q_{\Psi, 0^{-}}^{r}$ orbits on $X_{\Psi}$ are in one to one correspondence with the integrable orbits $G_{0}(x)$ such that $\mathcal{P}_{x}+\tau \odot_{x}$ is conjugate to $\boldsymbol{P}_{\Psi}$.

4. Let $K_{\Psi}$ be a maximal compact subgroup of $Q_{\Psi, 0}^{r}$ such that $\varkappa_{\Psi}$ contains the compact part of $\mathcal{H C}_{\Psi, 0}$ and is orthogonal to the noncompact part. Then the integrable orbits $G_{0}(x) \subset X$ such that $P_{x}+\tau P_{x}$ is conjugate to $P_{\Psi}$ are enumerated by the double coset space

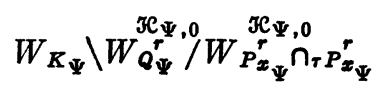

where superscript $\mathcal{H}_{\Psi, 0}$ means the subgroup of the Weyl group which preserves $\mathfrak{H}_{\Psi, 0}$ in $\mathfrak{H C}_{\Psi}$.

Proof. Statement (1) is the construction employed in proving that (2a) implies (2b) in Theorem 7.7.

Let $P_{z}+\tau P_{z}$ be conjugate to $P_{\Psi}$. Then $Q_{\Psi}$ and $P_{z}+\tau P_{z}$ are parabolic subalgebras of $\mathcal{S}$ which are conjugate by an element of $G$ and are defined/R; so they are conjugate by an element of $G_{0}$. Replacing $z$ by $g(z)$ for some $g \in G_{0}$ we may assume $\odot_{z}+\tau \odot_{z}=Q_{\Psi}$. Further replacing $z$ 
by $g(z)$ for some $g \in G_{0}$ we may also assume $\mathfrak{H C}_{\Psi, 0} \subset \mathcal{P}_{z}$. Let $\mathbb{B}_{2}$ be a Borel subalgebra of $\mathcal{P}_{z}^{r} \cap \tau \mathcal{P}_{z}^{r}$ containing $\mathcal{H}_{\Psi}$ such that $\mathbb{B}_{2}+\tau B_{2}=\mathscr{P}_{z}^{r}$ $\cap \tau \mathscr{P}_{z}^{r}$; such $\mathbb{B}_{2}$ exist because $\mathcal{H}_{\Psi, 0}$ is maximally compact in $\mathcal{G}_{0} \cap \mathcal{Q}_{\Psi}^{r}$, hence in $\mathcal{G}_{0} \cap\left(\mathcal{P}_{z}^{r} \cap \tau \mathcal{P}_{z}^{r}\right)$. Now $\mathbb{B}=\mathbb{B}_{2}+\left(\mathcal{P}_{z}^{r} \cap \tau \mathcal{P}_{z}^{-u}\right)+\mathcal{P}_{z}^{u}$ is a Borel subalgebra of $\mathcal{G}$ such that $B \subset \mathcal{P}_{z} \subset Q_{\Psi}$ and $B+\tau\left(\supset \mathcal{P}_{z}\right.$. As $P_{z}+\tau P_{z}=Q_{\Psi}$ now $B+\tau B=Q_{\Psi}$. Decompose $B=B_{1}+Q_{\Psi}^{u}$ where $B_{1}$ is a Borel subalgebra of $Q_{\Psi}^{r}$ containing $\mathcal{H}_{\Psi}$; now $\bigotimes_{1}+\tau B_{1}=Q_{\Psi}^{r}$. Thus, having replaced $z$ by $g(z)$ for some $g \in G_{0}$, we end up with $z$ as one of the points $x \in X$ constructed in (1). In other words, we started with $z \in G_{0}(x)$. Statement (2) is proved.

The choice available in the construction of statement (1) is the choice of $B_{1}$. That choice is free, without changing $G_{0}(x), Q_{\Psi}$ or $\mathcal{H}_{\Psi, 0}$, up to conjugation by an element of $Q_{\Psi, 0}$ which normalizes $\mathcal{H}_{\Psi, 0}$, i.e. up to conjugation by an element of $G_{0} \cap Q_{\Psi}^{r}$, i.e. up to conjugation by an element of $Q_{\Psi, 0}^{*}$. This proves (3), and (4) follows from Theorem 4.9. Q.E.D.

Theorems 7.7 and 7.10 give a complete description of the integrable $G_{0}$-orbits on $X$ in terms of the isotropy subalgebras of $\mathcal{G}$ at integrable points. But they do not "locate" the integrable orbits on $X$.

The complex flag manifold $X=G / P$ always has some integrable $G_{0}$-orbits, for at the beginning of this $\$ 7$ we observed that $P_{x}+\tau P_{x}=\mathcal{G}$ if and only if $G_{0}(x)$ is open in $X$. But it can happen that the open orbits are the only integrable $G_{0}$-orbits:

7.11. Proposition. Let $X=G / P$ be a complex flag manifold where $P$ is a maximal parabolic subgroup $p^{6}$ of $G$. Let $x \in X$ and let $G_{0}$ be a real form of $G$. Then the orbit $G_{0}(x)$ is integrable if and only if

(a) $G_{0}(x)$ is open in $X$, or

(b) $G_{0}(x)$ is the closed orbit and $\operatorname{dim}_{R} G_{0}(x)=\operatorname{dim}_{C} X$.

Proof. Let $G_{0}(x)$ be integrable. If $\tau P_{x}=\rho_{x}$ we are in case (b). If $\tau \mathcal{P}_{x} \neq \mathcal{P}_{x}$ then $\mathcal{P}_{x}+\tau \mathcal{P}_{x}=\mathcal{G}$ by maximality of $P$, so we are in case (a).

Q.E.D.

7.12. Corollary. Let $X_{0}=G_{0} / K$ be a hermitian symmetric space of noncompact type, $G=G_{0}^{\mathrm{e}}$, and $X=G_{u} / K=G / P$ the compact dual of $X$. Decompose $X_{0}$ as a product of irreducible factors $X_{0}^{(i)}=G_{0}^{(i)} / K^{(i)}, G$ as a product of simple groups $G^{(i)}=G_{0}^{(t) C}$, and $X$ as a product of irreducible factors $X^{(i)}=G_{u}^{(i)} / K^{(i)}=G^{(i)} / P^{(i)}$, so that the $G_{0}$-orbits on $X$ are just the products of $G_{0}^{(i)}$-orbits on the $X^{(i)}$. Let $x=\left(x_{1}, \cdots, x_{t}\right) \in X^{(1)} \times \cdots$

6 This means that $P \neq G$ and there is no parabolic subgroup $Q \subset G$ with $P_{\neq}^{\complement} Q_{\neq}^{\complement} G$. In other words, if $P=\mathcal{P}_{\Phi}$ where $\Phi$ is in a simple root system $\Pi$, then $|\Pi|=|\Phi|+1$. 
$X X^{(t)}=X$. Then the orbit $G_{0}(x) \subset X$ is integrable if, and only if, for each $i$,

either $G_{0}^{(i)}\left(x_{i}\right)$ is open in $X^{(i)}$

or $X_{0}^{(i)}$ is of tube type and $G_{0}^{(i)}\left(x_{i}\right)$ is its Bergman-Silov boundary in $X^{(i)}$.

Proof. We may assume $X_{0}$ irreducible. Then $t=1$ and $P$ is a maximal parabolic subgroup of $G$, and the two cases of Corollary 7.12 correspond to the respective cases (a) and (b) of Proposition 7.11.

Q.E.D.

\section{Chapter II. Decomposition OF A REAL GROUP ORBit INTO COMPLEX ANALYTIC PIECES}

Let $X=G / P$ be a complex flag manifold and let $G_{0}$ be a real form of $G$. $\$ 8$ and $\$ 9$ are an analysis of the "amount" of complex structure inherited from $X$ by an arbitrary orbit $G_{0}(x) \subset X$.

In $\$ 8$ the orbit $G_{0}(x)$ is decomposed into "holomorphic arc components" $S_{[z]}$. They are equivalence classes for the $G_{0}$-invariant relation: $y \in S_{[z]}$ if and only if it can be joined to $z$ by a chain of holomorphic (in $X$ ) arcs in $G_{0}(x)$. The holomorphic arc components are real analytic submanifolds of $G_{0}(x)$. In fact,

$$
N_{[z], 0}=\text { identity component of }\left\{g \in G_{0}: g S_{[z]}=S_{[z]}\right\}
$$

is a closed analytic subgroup of $G_{0}$ that is transitive on $S_{[z]}$. We approach analysis of the holomorphic arc components by studying the algebra $\mathfrak{N}_{[z]}=\mathfrak{N}_{[z], 0}^{\mathfrak{e}}$.

Theorem 8.5, Proposition 8.7 and Theorem 8.9 determine subspaces $Q_{[z]} \subset \mathscr{N}_{[z]} \subset\left\{\mathscr{N}_{[z]} \cap\left(P_{z}+\tau \odot_{z}\right)\right\}$ stable under $\tau$, such that $Q_{[z]}$ is a parabolic subalgebra of $\mathcal{G}$ (so $N_{[z], 0}$ is the identity component of a parabolic subgroup of $G_{0}$ ), $S_{[\varepsilon]}$ is a complex submanifold of $X$ if and only if $\mathfrak{T}_{[z]}=\mathfrak{T}_{[z]}$, and $\mathfrak{T}_{[z]}=\mathfrak{\Re}_{[z]}$ if and only if $\mathfrak{T}_{[z]}$ is an algebra. Thus one can decide whether the holomorphic arc components of $G_{0}(x)$ are complex submanifolds of $X$, and in that case their structure is determined in principle. If they are not complex then their submanifolds $Q_{[z], 0}(z) \subset S_{[z]}$, where $Q_{[z], 0}$ is the analytic group for $Q_{[z]} \cap g_{0}$, are acceptable substitutes which are complex submanifolds of $X$; we call them the "algebraic arc components" of $G_{0}(x)$.

If $P_{1} \subset P_{2}$ are parabolic subgroups of $G, X_{i}=G / P_{i}$ and $\pi: X_{1} \rightarrow X_{2}$ the projection, $x_{i} \in X_{i}$ with $\pi\left(x_{1}\right)=x_{2}$, then Proposition 8.14 relates the holomorphic and algebraic arc component structure of the $G_{0}\left(x_{i}\right)$.

$\$ 8$ ends with a description of the space of holomorphic arc components of $G_{0}(x)$. 
In $\S 9$ we study certain global conditions on the holomorphic arc components of $G_{0}(x)$. They are

(i) partially complex: $S_{[x]}$ is a complex manifold,

(ii) flag type: $N_{[x]}(x)$ is a complex flag manifold,

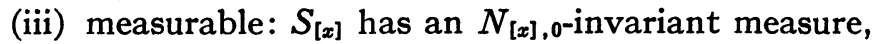

(iv) polarized: we can arrange $\mathfrak{F}$ so that $\tau \mathcal{P}_{x}^{r}=\mathcal{P}_{x}^{r}$,

(v) integrable: $\mathcal{P}_{x}+\tau \mathcal{P}_{x}$ is an algebra.

The important ones are "partially complex" and "measurable," and the others are useful in their study. Theorem 9.2 gives the basic analysis of measurable orbits, describing $\mathscr{N}_{[x]}$ and proving them partially complex and of flag type. Theorem 9.7 performs a similar task for orbits of flag type. Then Theorem 9.9 shows that, if $G_{0}(x)$ is polarized, then the conditions "measurable," "integrable," and "partially complex and of flag type" coincide. Various corollaries complete the general part of $\$ 9$.

The specific part of $\$ 9$ starts with Theorem 9.12, which says that the closed $G_{0}$-orbit on $X$ is automatically measurable. That result is related [12] to the existence of principal series representations of $G_{0}$. Proposition 9.18 and Corollary 9.21 extend the method of Theorem 9.12.

$\$ 9$ ends with a description of the case where $X$ is a hermitian symmetric space and $G_{0}$ is an arbitrary real form of $G$. Proposition 9.24 gives measurability criteria for the open $G_{0}$-orbits, and one criterion is that they be indefinite-kaehler symmetric spaces. The surprising corollary is that if the maximal compact subgroups of $G_{0}$ are of rank equal to that of $G_{0}$, then the open $G_{0}$-orbits on $X$ are indefinitekaehler symmetric spaces.

Notes for Chapter II. From August 1965 to May 1968, this author had a number of conversations with Bertram Kostant in which we tried to find the structure of the holomorphic arc components. We succeeded in the integrable case and saw that the normalizer of $\mathcal{P}_{x}^{u} \cap \tau \mathcal{P}_{x}^{u}$ should play a role. Statement 1 of Theorem 8.5 is due to Kostant, and the general case as worked out in $\$ 8$ was undoubtedly guided by the background of discussions with him.

Lemma 9.22 and Corollary 9.25 are due to Kostant.

Except for the basic contribution of Kostant described above, the material of $\S 8$ and $\$ 9$ was worked out by this author.

8. Holomorphic arc components. Given a complex flag manifold $X=G / P$ and a real form $G_{0}$ of $G$, the open $G_{0}$-orbits on $X$ are complex submanifolds. If $G_{0}(x) \subset X$ is the Borel embedding of an Hermitian symmetric space of noncompact type, then [15] one also knows that 
every $G_{0}$-orbit on the boundary of $G_{0}(x)$ has a natural decomposition into complex submanifolds of $X$. In this section we give an analogous decomposition of an arbitrary $G_{0}$-orbit into "holomorphic arc components," including an explicit criterion and characterization of the cases where those components are complex submanifolds of $X$.

Let $D$ be a subset of a complex analytic space $V$. By holomorphic $\operatorname{arc}$ in $D$ we mean a holomorphic map

$$
f:\{z \in \mathbf{C}:|z|<1\} \rightarrow V
$$

of the unit disc into $V$ such that $D$ contains the image of $f$. By chain of holomorphic arcs in $D$ we mean a finite sequence $\left\{f_{1}, \cdots, f_{k}\right\}$ of holomorphic arcs in $D$ such that the image of $f_{i}$ meets the image of $f_{i+1}$ for $1 \leqq i<k$.

8.1. Definition. Let $D$ be a subset of a complex analytic space $V$. Then the holomoprphic arc components of $D$ in $V$ are the equivalence classes of elements of $D$ under the relation: $v_{1} \sim v_{2}$ if there is a chain $\left\{f_{1}, \cdots, f_{k}\right\}$ of holomorphic arcs in D such that $v_{1}$ is in the image of $f_{1}$ and $v_{2}$ is in the image of $f_{k}$.

We draw some simple consequences from this definition.

8.2. Lemma. Let $V$ be a complex analytic space, let $A$ be a group of holomorphic diffeomorphisms of $V$, and let $D$ be an $A$-stable subset of $V$. Let $S$ be a holomorphic arc component of $D$ in $V$ and define $N_{A}(S)$ $=\{a \in A: a(S)=S\}$.

1. If $a \in A$ and $a(S)$ meets $S$, then $a(S)=S$ i.e. $a \in N_{A}(S)$.

2. If $D$ is an $A$-orbit then $N_{A}(S)$ is transitive on $S$.

3. If $A$ is a Lie transformation group on $V$, and if $D$ is an A-orbit, then $N_{A}(S)$ is a Lie subgroup of $A$, and $S$ and $D$ are embedded real submanifolds of $V$.

Proof. For (1) we have $s_{1}, s_{2} \in S$ with $a\left(s_{1}\right)=s_{2}$. If $s \in S$ we have a chain $\left\{f_{1}, \cdots, f_{k}\right\}$ of holomorphic arcs in $D$ from $s_{1}$ to $s$; now $\left\{a \cdot f_{1}, \cdots, a \cdot f_{k}\right\}$ is a chain of holomorphic arcs in $D$ from $a\left(s_{1}\right)=s_{2}$ to $a(s)$; thus $a(s) \in S$; that proves $a(S) \subset S$. Similarly $a^{-1}(S) \subset S$. Thus $a(S)=S$.

For (2) let $s_{1}, s_{2} \in S$. As $s_{i} \in D$ we have $a \in A$ with $a\left(s_{1}\right)=s_{2}$. Now $a \in N_{A}(S)$ by (1). Thus $N_{A}(S)$ has an element that sends $s_{1}$ to $s_{2}$.

For (3) we first note that $S$ is arcwise connected. Lifting arcs via $N_{A}(S) \rightarrow S$ we see that the identity component $N_{A}(S)^{0}$ is arcwise connected and is transitive on $S$; thus $N_{A}(S)^{0}$ is an analytic subgroup of $A$, so $N_{\Delta}(S)$ is a Lie subgroup, and $S$ is an embedded real submanifold of the embedded submanifold $D$. Q.E.D. 
8.3. Notation. Let $X=G / P$ be a complex flag manifold, $G_{0}$ a real form of $G$, and $x \in X$. Denote

(8.3a) $S_{[x]}$ : holomorphic arc component of $G_{0}(x)$ in $X$ that contains $x$.

(8.3b) $N_{[x], 0}$ : identity component of $\left\{g \in G_{0}: g S_{[x]}=S_{[x]}\right\}$.

(8.3c) $\mathscr{N}_{[x], 0}$ is the Lie algebra of $N_{[x], 0}$, and $\mathscr{N}_{[x]}=\mathfrak{K}_{[x], 0}^{\mathrm{e}}$.

(8.3d) $N_{[x]}$ is the complex analytic subgroup of $G$ for $\mathscr{T}_{[x]}$.

Lemma 8.2 says that $S_{[x]}$ is an embedded real submanifold of $X$, $N_{[x], 0}$ is a Lie subgroup of $G_{0}$, and $N_{[x], 0}$ is transitive on $S_{[x]}$. Note $\tau \mathscr{N}_{[x]}=\mathscr{N}_{[x]}$ by construction.

8.4. LeMMA. Let $X=G / P$ be a complex flag manifold, $G_{0}$ a real form of $G, x \in X, \mathfrak{H C} \subset \odot_{x} a \tau$-stable Cartan subalgebra of $\mathcal{G}$, and $\mathfrak{L}$ a complex subalgebra of $\mathcal{S}$ such that

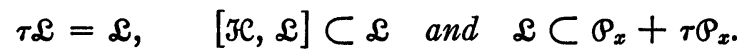

Let $\mathfrak{L}_{0}=\mathfrak{L} \cap \mathcal{G}_{0}$, real form of $\mathfrak{L}$; let $L$ and $L_{0}$ denote the analytic subgroups of $G$ and $G_{0}$ for $\mathfrak{L}$ and $\mathfrak{L}_{0}$.

1. $L_{0}(x)$ and $L(x)$ are complex submanifolds of $X$ with $L_{0}(x)$ open in $L(x)$.

2. $L_{0}(x) \subset S_{[x]}$.

3. $\mathfrak{L}_{0} \subset \mathscr{N}_{[x], 0}$ and $L_{0} \subset N_{[x], 0} ; \mathscr{L} \subset \mathscr{N}_{[x]}$ and $L \subset N_{[x]}$.

Proof. Choice of $\mathcal{H C}$ specifies $\mathcal{P}_{x}^{r}$ and $\mathcal{P}_{x}^{ \pm u}$. As $\mathscr{L}$ is complex and normalized by $\mathcal{H}$, it is $\mathscr{L} \cap \mathcal{H}$ plus certain root spaces; now $\mathscr{L} \subset\left(\mathcal{P}_{x}+\tau \mathcal{P}_{x}\right)$ shows

$\mathscr{L}=\mathscr{L}^{0}+\mathfrak{L}^{+}+\mathfrak{L}^{-}, \quad \mathscr{L}^{0}=\mathfrak{L} \cap\left(\mathcal{P}_{x} \cap \tau \odot_{x}\right), \quad \mathscr{L}^{+}=\mathfrak{L} \cap \tau \mathcal{P}_{x}^{-u} \subset \mathcal{P}_{x}$ and (using $\tau \mathscr{L}=\mathscr{L}$ )

$$
\mathscr{L}^{-}=\mathscr{L} \cap \mathcal{P}_{x}^{-u} \subset \tau \mathcal{P}_{x} \quad \text { with } \mathscr{L}^{-}=\tau \mathscr{L}^{+} .
$$

$L(x)$ is a complex submanifold of $X$ because $L$ is a complex Lie subgroup of $G$. Now

$$
\begin{aligned}
& \operatorname{dim}_{R} L_{0}(x)=\operatorname{dim}_{R} \mathscr{L}_{0}-\operatorname{dim}_{R} \mathfrak{L}_{0} \cap \mathcal{P}_{x} \\
& =\operatorname{dim}_{R} \mathscr{L}_{0}-\operatorname{dim}_{R} \mathscr{L}_{0} \cap \mathcal{P}_{x} \cap \tau \mathcal{P}_{x} \\
& =\operatorname{dim}_{\mathfrak{e}} \mathscr{L}-\operatorname{dim}_{\mathfrak{e}} \mathscr{L}^{0} \\
& =\operatorname{dim}_{\mathfrak{C}} \mathfrak{L}^{+}+\operatorname{dim}_{\mathfrak{C}} \mathfrak{L}^{-}=2 \operatorname{dim}_{\mathfrak{C}} \mathfrak{L}^{-} \\
& =2\left\{\operatorname{dim}_{\mathbb{e}} \mathfrak{L}-\operatorname{dim}_{\mathbb{e}} \mathfrak{L} \cap \boldsymbol{P}_{x}\right\} \\
& =2 \operatorname{dim}_{e} L(x)=\operatorname{dim}_{R} L(x)
\end{aligned}
$$

shows that $L_{0}(x)$ is open in $L(x)$. That proves (1). 
Covering by complex local coordinate neighborhoods one sees that $L_{0}(x)$ has just one holomorphic arc component. Thus $L_{0}(x) \subset S_{[x]}$, proving (2), and (3) follows immediately. Q.E.D.

We use Lemma 8.4 to prove $\mathfrak{I}_{[x]}$ parabolic by constructing a parabolic subalgebra $Q_{[x]} \subset \mathscr{N}_{[x]}$ of $\mathcal{G}$. That is the first step in the determination of $S_{[x]}$ in algebraic terms.

8.5. ThEOREM. Let $X=G / P$ be a complex flag manifold, $G_{0}$ a real form of $G, x \in X, \mathfrak{H C} \subset \odot_{x}$ a $\tau$-stable Cartan subalgebra of $\mathcal{G}$, and $\Phi$ a subset of a simple KC-root system such that $\mathcal{P}_{x}=\mathcal{P}_{\Phi}$.

Define a $\tau$-stable linear form $\delta_{\Phi}$ on अ by

$$
\delta_{\Phi}=\sum_{\Phi^{u} n_{\tau} \Phi^{u}} \varphi .
$$

Define $\tau$-stable sets of $\mathcal{H}$-roots of $\mathrm{G}$ by

$$
\Gamma^{r}=\left\{\varphi \in \Delta:\left\langle\varphi, \delta_{\Phi}\right\rangle=0\right\}, \quad \Gamma^{u}=\left\{\varphi \in \Delta:\left\langle\varphi, \delta_{\Phi}\right\rangle>0\right\},
$$

$$
\Gamma^{*}=\Gamma^{r} \cup \Gamma^{u} \text {. }
$$

Define a $\tau$-stable parabolic subalgebra $Q_{[x]} \subset \mathcal{G}$ by

$$
Q_{[x]}^{r}=x C+\sum_{r^{r}} \oint_{\varphi}, \quad Q_{[x]}^{u}=\sum_{r^{u}} \oint_{\varphi}, \quad Q_{[x]}=Q_{[x]}^{r}+Q_{[x]}^{u} .
$$

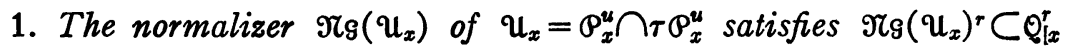
and $\mathfrak{T}_{g}\left(\mathcal{U}_{x}\right)^{u} \subset Q_{[x]}^{u}$.

2. $\mathcal{u}_{x} \subset \mathfrak{Y s}_{\mathcal{S}}\left(\mathcal{u}_{x}\right) \subset Q_{[x]} \subset\left\{\mathfrak{\vartheta}_{[x]} \cap\left(\mathcal{\odot}_{x}+\tau \odot_{x}\right)\right\}$.

3. $\Re_{[x]}$ is a parabolic subalgebra of $\mathcal{G}$ and $N_{[x], 0}$ is the identity component of the parabolic subgroup $N_{[x]} \cap G_{0}$ of $G_{0}$.

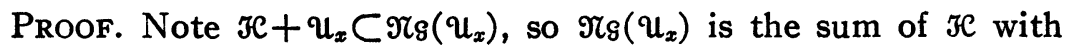
certain root spaces. Let $\varphi$ be a rootwith $\mathcal{G}_{\varphi} \subset \mathfrak{r}_{\rho}\left(\mathcal{U}_{x}\right)$, let $\oint[\varphi]$ denote the three dimensional simple algebra $h_{\varphi} \mathrm{C}+\oint_{\varphi}+\oint_{-\varphi}$, and let $\mathcal{Z}$ denote the centralizer of $g[\varphi]$ in $g$. Then

$$
Z=\{h \in \mathfrak{F}: \varphi(h)=0\}+\sum_{\psi \Perp \varphi} \mathcal{S}_{\psi}
$$

where $\psi \stackrel{\|}{=} \varphi$ denotes strong orthogonality, i.e. denotes that neither of $\varphi \pm \psi$ is a root. And further

$$
\mathcal{S}=\mathcal{Z}+\sum \mathfrak{N}_{i}, \quad \mathfrak{M}_{1}=\mathrm{g}[\varphi],
$$

where the $\Re_{i}$ are the nontrivial irreducible $S[\varphi]$-modules under ads. Thus for $i \neq 1$ we have $\mathfrak{N T}_{i}$ of the form 


$$
\mathfrak{T}_{i}=\sum_{j=-r}^{s} S_{\psi+j \varphi}, \quad \psi \in \Delta, \quad \frac{2\langle\psi, \varphi\rangle}{\langle\varphi, \varphi\rangle}=r-s,
$$

for the maximal string $S_{\psi}=\{\psi+j \varphi\}_{-r \leq j \leq s}$ of roots.

Suppose that $S_{\psi}$ meets $\Phi^{u} \cap \tau \Phi^{u}$, say $\psi+j_{0} \varphi \in \Phi^{u} \cap \tau \Phi^{u}$. Then $\mathcal{S}_{\varphi} \subset \Re g\left(u_{x}\right)$ implies $\psi+j \varphi \in \Phi^{u} \cap \tau \Phi^{u}$ for $j_{0} \leqq j \leqq s$, so we have an integer $j_{\psi},-r \leqq j_{\psi} \leqq s$, such that $S_{\psi} \cap\left(\Phi^{u} \cap \tau \Phi^{u}\right)=\left\{\psi+j \varphi: j_{\psi} \leqq j \leqq s\right\}$. Note $S_{\psi} \subset \Phi^{u} \cap \tau \Phi^{u}$ if and only if $j_{\psi}=-r$. But $j^{\prime}>j^{\prime \prime}$ implies $\left.\left\langle\varphi, \psi+j^{\prime} \varphi\right\rangle\right\rangle\left\langle\varphi, \psi+j^{\prime \prime} \varphi\right\rangle$ because $\langle\varphi, \varphi\rangle>0$; and $\operatorname{ad}\left(h_{\varphi}\right)$ has trace 0 $=\left\langle\varphi, \sum_{-r}^{s}(\psi+j \varphi)\right\rangle$ on $\mathfrak{N}_{i}$ because $\mathcal{G}[\varphi]$ is semisimple; thus

either $S_{\psi} \subset \Phi^{u} \cap \tau \Phi^{u}$ and $\left\langle\varphi, \sum_{\left.S_{\psi} \cap_{\Phi^{u}} \cap_{\tau} \Phi^{u} \gamma\right\rangle=0}\right.$

or $S_{\psi} \nsubseteq \Phi^{u} \cap \Phi^{u} \Phi^{u}$ and $\left\langle\varphi, \sum_{\left.S_{\psi} \cap \Phi^{u} n_{\tau} \Phi^{u} \gamma\right\rangle} \gamma\right\rangle$.

On the other hand, $\mathcal{S}_{-\varphi} \subset \Re_{\mathcal{S}}\left(\mathcal{u}_{x}\right)$ if and only if $\mathcal{G}_{-\varphi}$ normalizes each $\mathcal{u}_{x} \cap \mathscr{T}_{i}$, and, as above, that is equivalent to $S_{\psi} \subset \Phi^{u} \cap \tau \Phi^{u}$ whenever $S_{\psi}$ meets $\Phi^{u} \cap \tau \Phi^{u}$. Thus for $\varphi \notin \Phi^{u} \cap \tau \Phi^{u}$, we have $\left\langle\varphi, \delta_{\Phi}\right\rangle=0$ if $\mathcal{S}_{\varphi} \subset \Re_{g}\left(\mathcal{u}_{x}\right)^{r}$ and $\left\langle\varphi, \delta_{\Phi}\right\rangle>0$ if $\mathcal{G}_{\varphi} \subset \Re_{g}\left(\mathcal{u}_{x}\right)^{u}$. And for $\varphi$ in $\Phi^{u} \cap \tau \Phi^{u}$ we have $\mathcal{G}_{\varphi} \subset \Re_{g}\left(\mathcal{u}_{x}\right)^{u}$ with $\left\langle\varphi, \delta_{\Phi}\right\rangle>0$. That proves $\Re_{g}\left(\mathcal{u}_{x}\right)^{u} \subset Q_{[x]}^{u}$ and $\Re g\left(u_{x}\right)^{r} \subset Q_{[x]}^{r}$.

We have just proved $u_{x} \subset \Re g\left(u_{x}\right) \subset Q_{[x]}$. And $u_{x} \subset \Re g\left(u_{x}\right)^{u} \subset Q_{[x]}^{u}$ proves $\Theta_{[x]} \subset \mathcal{P}_{x}+\tau \mathcal{P}_{x}$. As $\mathfrak{H C} \subset Q_{[x]}=\tau Q_{[x]}$, Lemma 8.4 says $Q_{[x]} \subset \mathcal{N}_{[x]}$. That proves (2), and (3) follows because $Q_{[x]}$ is parabolic in G. Q.E.D.

The next step in the algebraic determination of $S$ consists of expanding $Q_{[x]}$ out toward $\mathscr{\Upsilon}_{[x]}$ as follows.

8.7. Proposition. Let $X=G / P$ be a complex flag manifold, $G_{0}$ a real form of $G, x \in X, \mathfrak{H} \subset \mathcal{P}_{x} a \tau$-stable Cartan subalgebra of $\mathcal{G}$, and $\Phi$ a subset of a simple HC-root system such that $P_{x}=P_{\Phi}$. Retain the notation (8.6). Define a $\tau$-stable subset $\Gamma^{0} \subset\left(\Phi^{*} \cup \tau \Phi^{*}\right) \backslash \Gamma^{*}$ by

(8.8a) $\Gamma^{0}=\left\{\varphi \in \Delta:-\varphi \notin \Phi^{u} \cap \tau \Phi^{u},\left\langle\varphi, \delta_{\Phi}\right\rangle<0, \varphi+\tau \varphi \notin \Delta\right\}$.

Define a $\tau$-stable subspace $\mathfrak{T C}_{[x]}$ of $\mathcal{G}_{0}$ by

$$
\mathfrak{M}_{[x]}=Q_{[x]}+\sum_{\Gamma^{0}} \oint_{\varphi}
$$

1. $Q_{[x]} \subset \mathscr{T}_{[x]} \subset \mathscr{N}_{[x]} \cap\left(\mathcal{P}_{x}+\tau \mathcal{P}_{x}\right)$.

2. If $\varphi \in \Gamma^{\circ}$, then $-\varphi \neq \tau \varphi \neq \varphi$, and either

(2a) $\varphi \perp \tau \varphi, \tau \varphi-\varphi \notin \Delta$, and $h_{\varphi} \mathrm{C}+h_{\tau \varphi} \mathrm{C}+\mathcal{G}_{\varphi}+\mathcal{G}_{-\varphi}+\mathcal{G}_{\tau \varphi}+\mathcal{G}_{-\tau \varphi}$ is $a$ $\tau$-stable subalgebra of type $A_{1} \oplus A_{1}$ of $\mathcal{G}$ contained in $\mathscr{N}_{[x]} \cap\left(\odot_{x}+\tau \odot_{x}\right)$; or

(2b) $\langle\varphi, \tau \varphi\rangle=\frac{1}{2}\langle\varphi, \varphi\rangle, \tau \varphi-\varphi \in \Delta$, and $h_{\varphi} \mathrm{C}+h_{\tau \varphi} \mathrm{C}+\mathcal{G}_{\varphi}+\mathcal{G}_{-\varphi}+\mathcal{G}_{\tau \varphi}$ $+\mathcal{G}_{-\tau \varphi}+\mathcal{G}_{\tau \varphi-\varphi}+\mathcal{G}_{\varphi-\tau \varphi}$ is a $\tau$-stable subalgebra of type $A_{2}$ in $\mathcal{G}$ contained in $\mathcal{T}_{[x]} \cap\left(\odot_{x}+\tau \odot_{x}\right)$.

3. $\mathfrak{N}_{[x]} \subset \mathcal{P}_{x}+\tau \mathcal{P}_{x}$ if and only if $\mathfrak{N}_{[x]}=\mathfrak{T}_{[x]}$. 
PROof. Decompose the real form $\mathfrak{H}_{0}=\mathfrak{H C} \cap \mathcal{G}_{0}$ of $\mathfrak{H C}$ as $\mathfrak{H C}_{0}=\mathfrak{H}_{T}+\mathfrak{H}_{V}$ such that $i \mathcal{F}_{T}+\mathcal{H}_{V}$ is the real span of the roots. If $\varphi \in \Delta$ then $\varphi=\varphi_{T}$ $+\varphi_{V}$ where $\varphi_{T}\left(\mathcal{F C}_{V}\right)=0=\varphi_{V}\left(\mathcal{F C}_{T}\right)$, and then $\tau \varphi=-\varphi_{T}+\varphi_{V}$. Now suppose $\left\langle\varphi, \delta_{\Phi}\right\rangle<0$ with $-\varphi \notin \Phi^{u} \cap \tau \Phi^{u}$. If $\tau \varphi=\varphi$ then $\mathcal{S}_{\varphi} \subset \mathcal{P}_{x}+\tau \mathcal{P}_{x}$ implies $\mathcal{G}_{\varphi} \subset \mathcal{P}_{x} \cap \tau \mathcal{P}_{x} \subset \Re{ }_{\mathcal{S}}\left(\mathcal{U}_{x}\right)$, so $\left\langle\varphi, \delta_{\Phi}\right\rangle \geqq 0$, contradicting $\left\langle\varphi, \delta_{\Phi}\right\rangle<0$. If $\tau \varphi=-\varphi$ then $\left\langle\tau \varphi, \delta_{\Phi}\right\rangle=\left\langle\tau \varphi, \tau \delta_{\Phi}\right\rangle=\left\langle\varphi, \delta_{\Phi}\right\rangle=-\left\langle\tau \varphi, \delta_{\Phi}\right\rangle$, contradicting $\left\langle\varphi, \delta_{\Phi}\right\rangle\left\langle 0\right.$. Thus $-\varphi \neq \tau \varphi \neq \varphi$, i.e. $\varphi_{T} \neq 0 \neq \varphi_{V}$. In particular $|\langle\varphi, \tau \varphi\rangle|$ $\langle|\langle\varphi, \varphi\rangle|$ so the integer $2\langle\varphi, \tau \varphi\rangle /\langle\varphi, \varphi\rangle=2\langle\varphi, \tau \varphi\rangle /\langle\tau \varphi, \tau \varphi\rangle$ is -1 , 0 or +1 .

Suppose further that $\varphi+\tau \varphi$ is not a root, i.e. that $\varphi \in \Gamma^{0}$. Then the maximal chain $\{\varphi+j \tau \varphi\}_{-r \leq j s 。}$ of roots has $s=0$, so $2\langle\varphi, \tau \varphi\rangle /\langle\tau \varphi, \tau \varphi\rangle$ $=r \geqq 0$, proving that $2\langle\varphi, \tau \varphi\rangle /\langle\varphi, \varphi\rangle$ is 0 (in which case $\varphi \perp \tau \varphi$ and $\tau \varphi-\varphi \notin \Delta$ ) or is 1 (in which case $\langle\varphi, \tau \varphi\rangle=\frac{1}{2}\langle\varphi, \varphi\rangle, \tau \varphi-\varphi \in \Delta$, and $\tau \varphi-2 \varphi \notin \Delta)$. Let $\&$ denote $h_{\varphi} \mathrm{C}+h_{\tau \varphi} \mathrm{C}+\mathcal{G}_{\varphi}+\mathcal{G}_{-\varphi}+\mathcal{G}_{\tau \varphi}+\mathcal{G}_{-\tau \varphi}$ in the first case, $h_{\varphi} \mathrm{C}+h_{\tau \varphi} \mathrm{C}+\mathcal{G}_{\varphi}+\mathcal{G}_{-\varphi}+\mathcal{G}_{\tau \varphi}+\mathcal{G}_{-\tau \varphi}+\mathcal{G}_{\varphi-\tau \varphi}+\mathcal{G}_{\tau \varphi-\varphi}$ in the second case. Then $\mathscr{L}$ is a $\tau$-stable subalgebra of type $A_{1} \oplus A_{1}$ or $A_{2}$ in $\mathcal{G}$, [अ, $\mathscr{L}] \subset \mathscr{L}$, and $\mathscr{L} \subset \mathcal{P}_{x}+\tau \odot_{x}$; the latter is obvious in the first case and follows from $\left\langle\tau \varphi-\varphi, \delta_{\Phi}\right\rangle=0=\left\langle\varphi-\tau \varphi, \delta_{\Phi}\right\rangle$ in the second case. Lemma 8.4 says $\mathscr{L} \subset \Re_{[x]}$. That completes the proof of (2), and (1) is an immediate consequence.

If $\Re_{[x]}=\mathscr{T}_{[x]}$, then $\mathfrak{T}_{[x]} \subset \mathcal{P}_{x}+\tau \mathcal{P}_{x}$ implies $\Re_{[x]} \subset \mathcal{P}_{x}+\tau \mathcal{P}_{x}$. Conversely suppose $\mathfrak{N}_{[x]} \subset \mathcal{P}_{x}+\tau \odot_{x}$. Then $\mathfrak{T}_{[x]} \subset \mathscr{N}_{[x]}$ implies that $\mathfrak{N}_{[x]}$ $=\mathfrak{N}_{[x]}+\sum_{A} \mathcal{S}_{\varphi}$ where $A$ is some $\tau$-stable set of roots such that

if $\alpha \in A$ then $-\alpha \notin \Phi^{u} \cap \tau \Phi^{u}, \quad\left\langle\alpha, \delta_{\Phi}\right\rangle<0$ and $\alpha+\tau \alpha \in \Delta$.

If $A$ is not empty, choose $\alpha \in A$ and observe that $\mathcal{S}_{\alpha+\tau \alpha} \subset\left[\mathfrak{N}_{[x]}, \Re_{[x]}\right]$ $\subset \Re_{[x]} \subset \mathcal{P}_{x}+\tau \mathcal{P}_{x}$, so $\tau(\alpha+\tau \alpha)=\alpha+\tau \alpha$ implies $\mathcal{S}_{\alpha+\tau \alpha} \subset \mathcal{P}_{x} \cap \tau \mathcal{P}_{x}$; that implies $\left\langle\alpha+\tau \alpha, \delta_{\Phi}\right\rangle \geqq 0$, contradicting

$$
\left\langle\alpha+\tau \alpha, \delta_{\Phi}\right\rangle=\left\langle\alpha, \delta_{\Phi}\right\rangle+\left\langle\tau \alpha, \tau \delta_{\Phi}\right\rangle=2\left\langle\alpha, \delta_{\Phi}\right\rangle<0 .
$$

Thus $A$ is empty and $\Re_{[x]}=\Re_{[x]}$. Q.E.D.

We apply Theorem 8.5 and Proposition 8.7 to the question of whether holomorphic arc components of $G_{0}(x)$ are complex submanifolds of $X$. Note that, in Theorem 8.9, (5) is the algebraic criterion for (1); it then specifies $\Re_{[x]}$ by (4), thus specifying $S_{[x]}=N_{[x], 0}(x)$ $\cong N_{[x], 0} /\left(P_{x} \cap \tau P_{x}\right)_{0}$.

8.9. Theorem. Let $X=G / P$ be a complex flag manifold, $G_{0}$ a real form of $G, x \in X, \mathfrak{H} \subset \odot_{x} a \tau$-stable Cartan subalgebra of $\mathcal{G}$, and $\Phi$ a subset

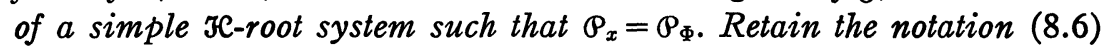
and (8.8). Then the following conditions are equivalent:

1. Every holomorphic arc component of $G_{0}(x)$ in $X$ is a complex submanifold of $X$. 
2. $S_{[x]}$ is a complex submanifold of $X$.

3. $\mathcal{T}_{[x]} \subset \mathcal{P}_{x}+\tau \boldsymbol{P}_{x}$.

4. $\mathscr{K}_{[x]}=\mathfrak{M}_{[x]}$, given by (8.6) and (8.8).

5. $\mathfrak{T}_{[x]}$ is an algebra.

Proof. (1) implies its special case (2). Conversely, if $g \in G_{0}$, then $g S_{[x]}=S_{[g x]}$, so (2) implies (1).

Lemma 8.4 says that (3) implies (2). For the converse we define a $G_{0}$-invariant distribution $T=\left\{T_{y}\right\}_{y \in G_{0}(x)}$ on $G_{0}(x)$ as follows. If $v \in \mathcal{G}$ then $v^{*}$ denotes the corresponding real tangent vector field on $X$, and its value at $y \in X$ is denoted $v_{v}^{*}$. Now define, for every $y \in G_{0}(x)$,

(8.10a) $T_{y}=\left\{v_{y}^{*}: v \in\left(\odot_{y}+\tau \odot_{y}\right) \cap g_{0}\right\}$, real subspace of $G_{0}(x)_{y}$.

We will prove that every holomorphic arc in $G_{0}(x)$ is tangent to $T$, i.e. that

(8.10b) if $f: D \rightarrow X$ is a holomorphic arc in $G_{0}(x)$ then $f_{*} D_{z} \subset T_{f(z)}$ for every $z \in D$.

Assume (8.10b). As (2) says that each tangent space to $S_{[x]}$ is filled out by the tangent spaces of holomorphic arcs in $G_{0}(x)$, it follows that $\left\{v_{y}^{*}: v \in \mathscr{N}_{[y], 0}\right\} \subset T_{y}$ for every $y \in G_{0}(x)$; it follows that $\mathscr{T}_{[x], 0}$

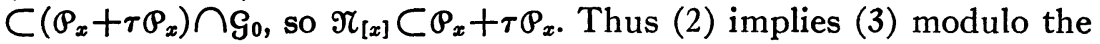
truth of $(8.10 \mathrm{~b})$.

We prove (8.10b). Let $z \in D, \beta \in D_{z}$. Let $J_{D}$ and $J_{X}$ be the almost complex structures on $D$ and $X$, so $f_{*}\left(J_{D} \beta\right)=J_{X} f_{*} \beta$ because $f$ is holomorphic. $f(D) \subset G_{0}(x)$ implies that both $f_{*} \beta$ and $f_{*} J_{D} \beta$ are in $G_{0}(x)_{f(z)}$. Thus we have $v \in \mathcal{S}_{0}$ with $f_{*} \beta=v_{f(z)}^{*}$, and $f_{*} J_{D} \beta=J_{X} f_{*} \beta=J_{X} v_{f(z)}^{*}$ $=(i v)_{f(z)}^{*}$. That says $i v \in \mathcal{S}_{0}+\boldsymbol{\rho}_{f(z)}$, say $i v=u+w$ where $u \in \mathcal{S}_{0}$ and $w \in P_{f(z)}$. Now $\tau v=v$ implies

$$
2 i v=i v-\tau(i v)=(u+w)-(\tau u+\tau w)=w-\tau w .
$$

Thus $2 i v \in \mathcal{P}_{f(z)}+\tau P_{f(z)}$, so $v \in \mathcal{G}_{0} \cap\left(P_{f(z)}+\tau P_{f(z)}\right)$, proving $f_{*} \beta=v_{f(z)}^{*}$ $\in T_{f(z)} \cdot(8.10 \mathrm{~b})$ is proved.

Proposition 8.7 contains the equivalence of (3) and (4). Summarizing now, we have proved $(8.10 \mathrm{~b})$ and have shown that (1), (2), (3) and (4) are equivalent. (4) implies (5) because $\Re_{[x]}$ is an algebra. Now we only need to prove that (5) implies (4).

Suppose that $\mathfrak{T l}_{[x]}$ is an algebra. Let $\mathfrak{T}_{[x], 0}$ denote its real form $\mathfrak{T}_{[x]} \cap \mathcal{G}_{0} ;$ let $M_{[x]}$ and $M_{[x], 0}$ denote the analytic subgroups of $G$ and $G_{0}$ for $\mathfrak{T}_{[x]}$ and $\mathfrak{T}_{[x], 0}$. Define $\mathfrak{T}_{[g x]}=\operatorname{ad}(g) \mathfrak{T}_{[x]}$, then $\mathfrak{T}_{[g x], 0}, M_{[g x]}$ and $M_{[g x], 0}$ as above, for every $g \in G_{0}$; those algebras and groups are well defined because $M_{[x], 0 \supset}\left(P_{x} \cap \tau P_{x}\right)_{0}=G_{0} \cap P_{x}$. Now we have a distribution $M=\left\{M_{y}\right\}$ on $G_{0}(x)$ given by

$$
M_{y}=\left\{v_{z}^{*}: v \in \mathfrak{N}_{[\nu], 0}\right\} \text {. }
$$


The distribution is integrable because $M_{y}$ is the tangent space to $M_{[y], 0}(y)$ at $y$. We will prove that every holomorphic arc in $G_{0}(x)$ is tangent to $M$. As $M$ is integrable, and as its integral manifolds $M_{[y], 0}(y)$ are complex submanifolds of $X$ by Lemma 8.4 and $M_{[g x], 0}(g x)=g M_{[x], 0}(x)$ for $g \in G_{0}$, we will conclude that the holomorphic arc components of $G_{0}(x)$ are complex submanifolds of $X$. Then (5) implies (4) and the theorem is proved.

Now we show that every holomorphic arc in $G_{0}(x)$ is tangent to $M$. As $M_{o(y)}=g_{*} M_{y}$ for $y \in G_{0}(x)$ and $g \in G_{0}$, it suffices to consider holomorphic arcs $f: D \rightarrow X$ in $G_{0}(x)$ with $f(0)=x$, and to prove $f_{*} D_{0} \subset M_{x}$. If $f_{*} D_{0}=0$ this is automatic. Now suppose $f_{*} D_{0} \neq 0$. The implicit function theorem says that $f$ maps a neighborhood of 0 diffeomorphically onto its image, so we cut $D$ down and assume that $f: D \rightarrow f(D)$ is a diffeomorphism. Let $\eta$ be a real vector field on $D$, let $J_{D}$ and $J_{X}$ be the almost complex structures on $D$ and $X$, and let $\xi=f_{*} \eta$. Then (8.10b) $\xi$ and $J_{X} \xi=f_{*} J_{D} \eta$ are tangent to $\left.T\right|_{f(D)}$, and so is $\left[\xi, J_{X} \xi\right]$ $=f_{*}\left[\eta, J_{D} \eta\right]$.

Given $v \in \mathcal{G}_{0}$, the vector field $v^{*}$ induced on $G_{0}(x)$ is induced from the 1-parameter group $\{\exp t v\} \subset G_{0}$ acting on $G_{0}(x)$; it is the projection of a right invariant vector field on $G_{0}$. Let $\sigma$ be a smooth local section of $G_{0} \rightarrow G_{0} /\left(P_{x} \cap \tau P_{x}\right)_{0}=G_{0}(x)$ in a neighborhood $W$ of $x$. If $v \in \mathcal{G}_{0}$, and if $\tilde{v}$ denotes the corresponding left invariant vector field on $G_{0}$, then $\left.\tilde{v}\right|_{\sigma(W)}$ projects to a vector field $v^{\dagger}$ on $W$. Observe

$$
\begin{aligned}
& T_{y}=\left\{v_{y}^{*}: v \in \mathcal{S}_{0} \cap\left(\mathcal{P}_{y}+\tau \mathcal{P}_{y}\right)\right\} \text { is }\left\{v_{y}^{\dagger}: v \in \mathcal{S}_{0} \cap\left(\mathcal{P}_{x}+\tau \mathcal{P}_{x}\right)\right\}, \\
& M_{y}=\left\{v_{y}^{*}: v \in \mathscr{M}_{[y], 0}\right\} \text { is }\left\{v_{y}^{\dagger}: v \in \mathscr{M}_{[x], 0}\right\} \text {, } \\
& N_{y}=\left\{v_{y}^{*}: v \in \mathscr{N}_{[y], 0}\right\} \text { is }\left\{v_{y}^{+}: v \in \mathscr{N}_{[x], 0}\right\},
\end{aligned}
$$

from $G_{0}$-invariance of the corresponding distributions. Now cut $W$ down if necessary, so that the tangent vector field $\xi$ to $f(D) \subset S_{[x]}$ $=N_{[x], 0}(x)$ has representation

$$
\xi_{y}=\zeta(y)_{y}^{\dagger} \text { where } \zeta: f(D) \cap W \rightarrow \mathfrak{\Re}_{[x], 0} \text { is smooth. }
$$

As $\xi$ is tangent to $T, \zeta$ takes values in $\left(\mathcal{P}_{x}+\tau \mathcal{P}_{x}\right) \cap \Re_{[x], 0}$.

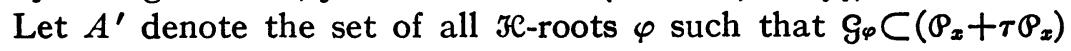
$\cap \Re_{[x]}$ but $\mathcal{G}_{\varphi} \subset \Re_{[x]}$. Then $\left(\mathcal{P}_{x}+\tau \mathcal{P}_{x}\right) \cap \Re_{[x]}=\Re_{[x]}+Q$ direct sum where $a=\sum_{A^{\prime}} \mathcal{G}_{\varphi}$, and $\zeta$ takes values in $\Re_{[x], 0}+a_{0}$ where $a_{0}=a \cap g_{0}$. Decompose $\zeta=\mu+\alpha$ where $\mu$ takes values in $\mathfrak{M}_{[x], 0}$ and $\alpha$ takes values in $a_{0}$. That decomposes $\xi=m+a$ and $J_{X} \xi=J_{X} m+J_{X} a$ where

$$
m_{y}=\mu(y)_{y}^{\dagger} \text { and } a_{y}=\alpha(y)_{y}^{\dagger} .
$$


Now

$$
\begin{aligned}
{\left[a, J_{X} a\right]_{x} } & =\left[\xi-m, J_{X} \xi-J_{X} m\right]_{x} \\
& =\left[\xi, J_{X} \xi\right]_{x}-\left[m, J_{X} \xi\right]_{x}-\left[\xi, J_{X} m\right]_{x}+\left[m, J_{X} m\right]_{x} .
\end{aligned}
$$

We have already seen $\left[\xi, J_{x} \xi\right]_{x} \in T_{x}$. We have $\left[m, J_{X} \xi\right]_{x} \in T_{x}$ and $\left[\xi, J_{X} m\right]_{x} \in T_{x}$ because $m$ and $J_{X} m$ are tangent to $M$ and $M_{[x], 0}$ preserves the real span of the tangent spaces to holomorphic arcs. And finally $\left[m, J_{x} m\right]_{x} \in M_{x} \subset T_{x}$ because $\mathfrak{T}_{[x], 0}$ is an algebra. Thus $\left[a, J_{X} a\right]_{x} \in T_{x}$.

$A^{\prime}=A \cup \tau A$ disjoint where $A=A^{\prime} \cap \tau^{*} \Phi^{*}=A^{\prime} \cap-\Phi^{u}$. If $\varphi \in A$ choose $0 \neq e_{\varphi} \in \mathcal{G}_{\varphi}$. Define $p_{\varphi}=e_{\varphi}+\tau e_{\varphi} \in \mathcal{S}_{0}$ and $q_{\varphi}=i e_{\varphi}+\tau\left(i e_{\varphi}\right)=i e_{\varphi}-i \tau e_{\varphi} \in \mathcal{S}_{0}$. As $\mathcal{P}_{x}^{-u}$ is the holomorphic tangent space to $X$ at $x, J_{X} p_{\varphi}^{\dagger}=q_{\varphi}^{\dagger}$ and $J_{X} q_{\varphi}^{\dagger}=-p_{\varphi}^{\dagger}$, for $\varphi \in A$, at $x$. Invariance then gives $J_{X} p_{\varphi}^{\dagger}=q_{\varphi}^{\dagger}$ and $J_{X} q_{\varphi}^{\dagger}$ $=-p_{\varphi}^{\dagger}$ on $G_{0}(x)$ for $\varphi \in A$. Now express $\alpha(y)=\sum_{A}\left(s_{\varphi}(y) p_{\varphi}+t_{\varphi}(y) q_{\varphi}\right)$, so that $a=\sum_{A}\left(s_{\varphi} p_{\varphi}^{\dagger}+t_{\varphi} q_{\varphi}^{\dagger}\right)$ and $J_{X} a=\sum_{A}\left(-t_{\varphi} p_{\varphi}^{\dagger}+s_{\varphi} q_{\varphi}^{\dagger}\right)$. Then we compute $\left[a, J_{\boldsymbol{X}} a\right]=\sum_{\varphi, \psi \in A}\left[s_{\varphi}^{\dagger} p_{\varphi}+t_{\varphi} q_{\varphi}^{\dagger},-t_{\psi} p_{\psi}^{\dagger}+s_{\psi} q_{\psi}^{\dagger}\right]$ and

$$
\begin{aligned}
{\left[s_{\varphi} p_{\varphi}^{\dagger}+t_{\varphi} q_{\varphi}^{\dagger},-t_{\psi} p_{\psi}^{\dagger}+s_{\psi} q_{\psi}^{\dagger}\right] } & \\
= & -s_{\varphi} \cdot p_{\varphi}^{\dagger}\left(t_{\psi}\right) \cdot p_{\psi}^{\dagger}+t_{\psi} \cdot p_{\psi}^{\dagger}\left(s_{\varphi}\right) \cdot p_{\varphi}^{\dagger}-s_{\varphi} t_{\psi}\left[p_{\varphi}, p_{\psi}\right]^{\dagger} \\
& +s_{\varphi} \cdot p_{\varphi}^{\dagger}\left(s_{\psi}\right) \cdot q_{\psi}^{\dagger}-s_{\psi} \cdot q_{\psi}^{\dagger}\left(s_{\varphi}\right) \cdot p_{\varphi}^{\dagger}+s_{\varphi} s_{\psi}\left[p_{\varphi}, q_{\psi}\right]^{\dagger} \\
& -t_{\varphi} \cdot q_{\varphi}^{\dagger}\left(t_{\psi}\right) \cdot p_{\psi}^{\dagger}+t_{\psi} \cdot p_{\psi}^{\dagger}\left(t_{\varphi}\right) \cdot q_{\varphi}^{\dagger}-t_{\varphi} t_{\psi}\left[q_{\varphi}, p_{\psi}\right]^{\dagger} \\
& +t_{\varphi} \cdot q_{\varphi}^{\dagger}\left(s_{\psi}\right) \cdot q_{\psi}^{\dagger}-s_{\psi} \cdot q_{\psi}^{\dagger}\left(t_{\varphi}\right) \cdot q_{\varphi}^{\dagger}+t_{\varphi} s_{\psi}\left[q_{\varphi}, q_{\psi}\right]^{\dagger} .
\end{aligned}
$$

As $\lambda \in A$ implies $p_{\lambda x}^{\dagger}, q_{\lambda x}^{\dagger} \in T_{x}$, and as $\left[a, J_{X} a\right]_{x} \in T_{x}$, it follows that

$$
\begin{aligned}
0 \equiv & {\left[a, J_{X} a\right]_{x} \bmod . T_{x} } \\
\equiv & \sum_{\varphi, \psi \in A}\left\{-s_{\varphi}(x) t_{\psi}(x)\left[p_{\varphi}, p_{\psi}\right]_{x}^{\dagger}+s_{\varphi}(x) s_{\psi}(x)\left[p_{\varphi}, q_{\psi}\right]_{x}^{\dagger}\right. \\
& \left.-t_{\varphi}(x) t_{\psi}(x)\left[q_{\varphi}, p_{\psi}\right]_{x}^{\dagger}+t_{\varphi}(x) s_{\psi}(x)\left[q_{\varphi}, q_{\psi}\right]_{x}^{\dagger}\right\} \bmod T_{x} \\
= & \sum_{\varphi, \psi \in A}\left\{-s_{\varphi}(x) t_{\psi}(x)\left(\left[e_{\varphi}, e_{\psi}\right]+\tau\left[e_{\varphi}, e_{\psi}\right]+\left[e_{\varphi}, \tau e_{\psi}\right]+\left[\tau e_{\varphi}, e_{\psi}\right]\right)_{x}^{\dagger}\right. \\
& +s_{\varphi}(x) s_{\psi}(x)\left(i\left[e_{\varphi}, e_{\psi}\right]-i \tau\left[e_{\varphi}, e_{\psi}\right]-i\left[e_{\varphi}, \tau e_{\psi}\right]+i\left[\tau e_{\varphi}, e_{\psi}\right]\right)_{x}^{\dagger} \\
& -t_{\varphi}(x) t_{\psi}(x)\left(i\left[e_{\varphi}, e_{\psi}\right]-i \tau\left[e_{\varphi}, e_{\psi}\right]+i\left[e_{\varphi}, \tau e_{\psi}\right]-i\left[\tau e_{\varphi}, e_{\psi}\right]\right)_{x}^{\dagger} \\
& \left.+t_{\varphi}(x) s_{\psi}(x)\left(-\left[e_{\varphi}, e_{\psi}\right]-\tau\left[e_{\varphi}, e_{\psi}\right]+\left[e_{\varphi}, \tau e_{\psi}\right]+\left[\tau e_{\varphi}, e_{\psi}\right]\right)_{x}^{\dagger}\right\} \\
= & -2 \sum_{\varphi, \psi \in A} s_{\varphi}(x) t_{\psi}(x)\left(\left[e_{\varphi}, \tau e_{\psi}\right]+\left[\tau e_{\varphi}, e_{\psi}\right]\right)_{x}^{\dagger} \\
& +\sum_{\varphi, \psi \in A}\left\{s_{\varphi}(x) s_{\psi}(x)+t_{\varphi}(x) t_{\psi}(x)\right\}\left\{i\left(\left[\tau e_{\varphi}, e_{\psi}\right]+\left[\tau e_{\psi}, e_{\varphi}\right]\right)_{x}^{\dagger}\right\}
\end{aligned}
$$


Let $\beta \in A$. Then $\left\langle\beta, \delta_{\Phi}\right\rangle<0,-\beta \notin \Phi^{u} \cap \tau \Phi^{u}$ and (because $\mathcal{G}_{\beta}\left\lceil\mathfrak{T l}_{[x]}\right.$ ) $\beta+\tau \beta$ is a root. If $\mathcal{G}_{\beta+\tau \beta} \subset \mathcal{P}_{x}+\tau \odot_{x}$, then $\tau \mathcal{G}_{\beta+\tau \beta}=\mathcal{G}_{\beta+\tau \beta}$ would imply $\mathcal{G}_{\beta+\tau \beta} \subset \mathcal{P}_{x} \cap \tau \mathcal{P}_{x}$; that would say $\left\langle\beta+\tau \beta, \delta_{\Phi}\right\rangle \geqq 0$, contradicting $\left\langle\beta+\tau \beta, \delta_{\Phi}\right\rangle=2\left\langle\beta, \delta_{\Phi}\right\rangle<0$. Thus $\mathcal{S}_{\beta+\tau \beta} \subset \mathcal{P}_{x}^{-u} \cap \tau \odot_{x}^{-u}$. Define $E_{\beta+\tau \beta}$ $=\left\{v_{x}^{\dagger}: v_{x} \in \mathcal{G}_{0} \cap \mathcal{G}_{\beta+\tau \beta}\right\}$, 2-dimensional real subspace of the tangent space to $G_{0}(x)$ at $x$. Then the projection of $\left[a, J_{X} a\right]_{x}$ to $E_{\beta+\tau \beta}$ is

$$
\begin{aligned}
0= & \left\{s_{\beta}(x)^{2}+t_{\beta}(x)^{2}\right\}\left\{2 i\left[\tau e_{\beta}, e_{\beta}\right]\right\}_{x}^{\dagger} \\
& +\sum_{\substack{\varphi \neq \psi \text { in } A \\
\varphi+\tau \psi=\beta+\tau \beta}}\left(\left\{i s_{\varphi}(x) s_{\psi}(x)+i t_{\varphi}(x) t_{\psi}(x)-2 s_{\varphi}(x) t_{\psi}(x)\right\}\left[\tau e_{\varphi}, e_{\psi}\right]\right)_{x}^{\dagger} \\
& +\sum_{\substack{\varphi \neq \psi \text { in } A \\
\varphi+\tau \psi=\beta+\tau \beta}}\left(\left\{i s_{\varphi}(x) s_{\psi}(x)+i t_{\varphi}(x) t_{\psi}(x)+2 s_{\varphi}(x) t_{\psi}(x)\right\}\left[\tau e_{\psi}, e_{\varphi}\right]\right)_{x}^{\dagger}
\end{aligned}
$$

Suppose that the holomorphic arc $f$ does not satisfy $f_{*} D_{0} \subset M_{x}$. Then we can choose $\eta$ such that $\xi_{x} \notin M_{x}$, i.e. such that $\alpha(x) \neq 0$, so there is a root $\beta \in A$ for which $s_{\beta}(x)^{2}+t_{\beta}(x)^{2} \neq 0$. Recall that $Q$ is disjoint from the parabolic subalgebra $Q_{[x]}$, in fact that $a \subset Q_{[x]}^{-u}$. So there is an ordering of the $\mathcal{H}$-roots of $\mathrm{g}$ such that $A$ consists of negative roots. Now choose $\beta$ to be the highest root in $A$ such that $s_{\beta}(x)^{2}$ $+t_{\beta}(x)^{2} \neq 0$, i.e. such that the real numbers $s_{\beta}(x)$ and $t_{\beta}(x)$ are not both zero. Now suppose $\varphi, \psi \in A$ with $\varphi \neq \psi$ and $\varphi+\tau \psi=\beta+\tau \beta$. Then either $s_{\varphi}(x)=0=t_{\varphi}(x)$ or $s_{\psi}(x)=0=t_{\psi}(x)$; for otherwise $\varphi<\beta$ and $\psi<\beta$, whence $\varphi+\tau \psi<\beta+\tau \beta$. Thus

$$
s_{\varphi}(x) s_{\psi}(x)=t_{\varphi}(x) t_{\psi}(x)=s_{\varphi}(x) t_{\psi}(x)=0,
$$

and our calculation above reduces to

$$
\left\{s_{\beta}(x)^{2}+t_{\beta}(x)^{2}\right\}\left\{2 i\left[\tau e_{\beta}, e_{\beta}\right]\right\}_{x}^{\dagger}=0 .
$$

As $\left\{i\left[\tau e_{\beta}, e_{\beta}\right]\right\}_{x}^{\dagger} \neq 0$ from $\tau \mathcal{S}_{\beta+\tau \beta}=\mathcal{G}_{\beta+\tau \beta}$, it follows that $s_{\beta}(x)^{2}+t_{\beta}(x)^{2}=0$, which contradicts our choice of $\beta$. We conclude that $f_{*} D_{0} \subset M_{x}$. That completes the proof of the theorem. Q.E.D.

8.11. Corollary. Let $X=G / P$ be a complex flag manifold, $G_{0}$ a real form of $G, x \in X, \mathfrak{s} \subset \mathcal{P}_{x} a \tau$-stable Cartan subalgebra of $\mathcal{G}$, and $\Phi$ a subset of a simple KC-root system such that $\odot_{x}=\odot_{\Phi}$. Then the following conditions are equivalent:

(i) $\mathscr{T}_{[x]}=Q_{[x]}$, parabolic subalgebra defined by (8.6).

(ii) $Q_{[x]}=\Re_{[x]}$, parabolic subalgebra defined by (8.8).

(iii) If $\varphi$ is an $\mathfrak{H}^{\mathrm{C}}$-root such that $\left\langle\varphi, \delta_{\Phi}\right\rangle<0$ and $-\varphi \notin \Phi^{u} \cap \tau \Phi^{u}$ then $\varphi+\tau \varphi$ is a root.

If those conditions hold, the holomorphic arc components of $G_{0}(x)$ are complex submanifolds of $X$. 
ProOF. (iii) implies (ii) by (8.8). Given (ii), $\mathfrak{T}_{[x]}$ is an algebra, so $\mathfrak{N}_{[x]}=\mathfrak{N}_{[x]}$ by Theorem 8.9 , whence (ii) says $\mathfrak{T}_{[x]}=\mathcal{Q}_{[x]}$. Given (i), $Q_{[x]} \subset \mathscr{N}_{[x]} \subset \Re_{[x]}$ implies (ii), and (iii) follows by (8.8). Q.E.D.

8.12. ExAmple. Let $G=\mathrm{SL}(2 m, \mathrm{C})$ and either $G_{0}=\mathrm{SL}(m, \mathrm{Q})$ with maximal compact subgroup $\mathrm{Sp}(m)$, or $G_{0}=\mathbf{S L}(2 m, \mathbf{R})$ with maximal compact subgroup SO $(2 m)$. Choose a $\tau$-stable Cartan subalgebra $\mathfrak{H C} \subset \mathcal{S}$ and a $\tau$-stable simple root system $\Psi$. Then $\tau$ acts on $\Psi$ by

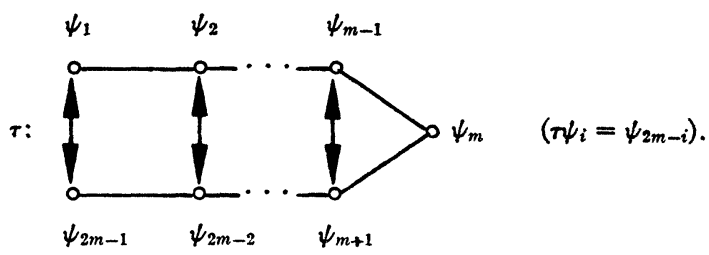

Let $\mathcal{P}=\mathcal{P}_{x}=\mathcal{P}_{\Phi}$ where $\Phi=\left\{\psi_{1}, \cdots, \psi_{2 m-2}\right\}$. Then

$$
X=G / P \text { is complex projective }(2 m-1) \text {-space, }
$$

and we note

$$
\begin{aligned}
\Phi^{u} & =\left\{\psi_{i}+\psi_{i+1}+\cdots+\psi_{2 m-1}: 1 \leqq i \leqq 2 m-1\right\}, \\
\tau \Phi^{u} & =\left\{\psi_{1}+\psi_{2}+\cdots+\psi_{j}: 1 \leqq j \leqq 2 m-1\right\},
\end{aligned}
$$

so

$\Phi^{u} \cap \tau \Phi^{u}=\{\mu\}$ where $\mu=\psi_{1}+\cdots+\psi_{2 m-1}$ maximal root.

Thus

$$
\delta_{\Phi}=\mu \quad \text { and } \quad Q_{[x]}=\rho_{\Gamma}, \quad \Gamma=\left\{\psi_{2}, \psi_{3}, \cdots, \psi_{2 m-2}\right\} .
$$

Now let $B$ consist of all roots $\varphi$ such that $\mathcal{G}_{\varphi} \subset \odot_{x}+\tau \odot_{x}$ and $\mathcal{G}_{\varphi} \subsetneq Q_{[x]}$. Then

$$
\begin{aligned}
B=\left\{-\left(\psi_{1}+\cdots+\psi_{i}\right),-\left(\psi_{j}+\cdots+\psi_{2 m-1}\right):\right. & \\
1 & \leqq i<2 m-1,1<j \leqq 2 m-1\} .
\end{aligned}
$$

If $\varphi \in B$, now $\varphi+\tau \varphi$ is of the form

$$
-\left[\left(\psi_{1}+\cdots+\psi_{i}\right)+\left(\psi_{2 m-i}+\cdots+\psi_{2 m-1}\right)\right],
$$

which cannot be a root because we cannot have $2 m-i=i+1$. Thus if $\varphi \in B$ then $\varphi+\tau \varphi$ is not a root.

In other words, $B=\Gamma^{0}$, so now

$$
\mathfrak{M}_{[x]}=\odot_{x}+\tau \odot_{x}=\mathscr{H}+\sum_{\varphi \neq-\mu} \mathcal{G}_{\varphi}, \quad \text { not an algebra. }
$$


Thus the holomorphic arc components of $G_{0}(x)$ are not complex submanifolds of $X$. In fact, as $Q_{[x]}$ is maximal among the $\tau$-stable subalgebras of $\mathcal{G}$, one expects that the $Q_{[y], 0}(y), y \in G_{0}(x)$, would be maximal among the connected complex submanifolds of $X$ that are contained in $G_{0}(x)$.

The example above is based on the situation that $\tau$ preserves a simple root system $\Psi$ but acts nontrivially on $\Psi$. The latter occurs if and only if $\tau$ induces an outer automorphism on the compact real form of $G$, i.e. if and only if the maximal compact subgroups $K$ of $G_{0}$ have rank $K<\operatorname{rank} G_{0}$. Later we will see, for the case where $X$ is a compact hermitian symmetric space and the inclusion $G_{0}\left(x_{0}\right) \subset X$ of some open orbit is the Borel embedding of the noncompact dual of $X$, that every holomorphic arc component of every $G_{0}$-orbit on $X$ is a hermitian symmetric submanifold (the signature of metric can be indefinite there). In particular, if $X$ is complex projective space as in the example, and if $G_{0}$ is not one of the real forms of $G$ considered above, then it will follow that every holomorphic arc component of every $G_{0}$-orbit is a complex submanifold of $X$.

8.13. Lemma/Definition. Let $X=G / P$ be a complex flag manifold, $G_{0}$ a real form of $G, x \in X, \mathfrak{H} \subset \odot_{x} a \tau$-stable Cartan subalgebra of $\mathcal{G}$, and $\Phi$ a subset of a simple $\mathcal{F}$-root system such that $\mathcal{P}_{x}=\mathcal{P}_{\Phi}$. Let $\mathcal{Q}_{[x]}$ be the $\tau$-stable parabolic subalgebra of $\mathcal{G}$ defined by (8.6), $\mathcal{Q}_{[x], 0}$ the real form $Q_{[x]} \cap g_{0}$ of $Q_{[x]}$, and $Q_{[x], 0}$ the analytic subgroup of $G_{0}$ for $Q_{[x], 0}$. Then

(i) $Q_{[x], 0}(x) \subset S_{[x]}$ is a complex submanifold of $X$,

(ii) $Q_{[x]}, Q_{[x], 0}$ and $Q_{[x], 0}(x)$ are independent of choices of $\mathfrak{H C}$ and $\Phi$,

(iii) if $y \in Q_{[x], 0}(x)$ then $Q_{[y], 0}(y)=Q_{[x], 0}(x)$, and

(iv) if $g \in G_{0}$ then $Q_{[g x], 0}(g x)=g Q_{[x], 0}(x)$.

We will refer to the $Q_{[y], 0}(y), y \in G_{0}(x)$, as the algebraic arc components of $G_{0}(x)$.

REMARK. Lemma 8.13 says that the algebraic arc components of $G_{0}(x)$ enjoy the same formal properties as the holomorphic arc components. Example 8.12 shows that they are the best substitute for the holomorphic arc components in case the latter are not complex submanifolds of $X$. Corollary 8.11 gives the criterion for the algebraic arc components of $G_{0}(x)$ to coincide with the holomorphic arc components.

Proof. Assertion (i) is immediate from Lemma 8.4.

Suppose that $Q_{[x], 0}(x)$ does not depend on the choices of $\mathscr{F}$ and $\Phi$. If $g \in G_{0}$, then we define $Q_{[x x], 0}(g x)$ from the choices ad $(g) \operatorname{rC} C \operatorname{ad}(g) P_{x}$ $=\mathcal{Q}_{\rho x}$ and $\operatorname{ad}\left(g^{-1}\right) * \Phi$; then $Q_{[g x]}=\operatorname{ad}(g) Q_{[x]}$, so $Q_{[g x], 0}=\operatorname{ad}(g) Q_{[x], 0}$, implying the statement $Q_{[o x], 0}(g x)=g \cdot Q_{[x], 0}(x)$ of (iv). If $g \in Q_{[x], 0}$ we obtain $Q_{[s x], 0}=Q_{[x], 0}$, so $Q_{[x x], 0}(g x)=Q_{[x], 0}(g x)=Q_{[x], 0}(x)$, proving (iii). Thus we are reduced to proving (ii). 
Given $\mathfrak{H C}, \delta_{\Phi}=\sum_{\Phi}{ }^{u} n_{\tau} \Phi^{u} \varphi=\sum_{-\Phi}{ }^{u} n_{-\tau \Phi}{ }^{u}(-\psi)$ is the sum of all $\mathfrak{H C}$ roots $-\psi$ such that $\mathcal{S}_{\psi}\left\lceil\rho_{x}+\tau \odot_{x}\right.$; thus $\delta_{\Phi}, Q_{[x]}, Q_{[x], 0}$ and $Q_{[x], 0}(x)$ are independent of choice of the set $\Phi$ for which $\rho_{x}=\rho_{\Phi}$, and they depend only on the choice of $\mathcal{F}$.

Let $\mathcal{H C}^{\prime}$ and $\mathcal{H C}^{\prime \prime}$ be two choices of $\mathcal{H C}$. They are $\tau$-stable Cartan subalgebras of respective reductive parts $\left(\mathcal{P}_{x} \cap \tau \odot_{x}\right)^{\prime}$ and $\left(\mathcal{P}_{x} \cap \tau \odot_{x}\right)^{\prime \prime}$ of $\odot_{x} \cap \tau \odot_{x}$. Let $Q_{[x]}^{\prime}$ and $Q_{|x|}^{\prime \prime}$ be the analytic subgroups of $G$ for the corresponding algebras $Q_{[x]}^{\prime}$ and $Q_{[x]}^{\prime \prime}$; let $L^{\prime}, L^{\prime \prime}$ and $L$ denote the respective analytic subgroups of $G$ for $\left(\odot_{x} \cap \tau \odot_{x}\right)^{\prime},\left(\odot_{x} \cap \tau \odot_{x}\right)^{\prime \prime}$ and $\left(P_{x} \cap \tau \odot_{x}\right)$. Then $\left(L^{\prime} \cup L^{\prime \prime}\right) \subset L \subset\left(Q_{[x]}^{\prime} \cap Q_{[x]}^{\prime \prime}\right)$. As any two reductive parts of $L$ are conjugate, we have $g_{1} \in L$ such that $\operatorname{ad}\left(g_{1}\right) L^{\prime \prime}=L^{\prime}$; as any two Cartan subgroups of $L^{\prime}$ are conjugate, we have $g_{2} \in L^{\prime}$ such that $\operatorname{ad}\left(g_{2} g_{1}\right) \mathcal{H C}^{\prime \prime}$ $=\mathfrak{F C}^{\prime}$. Now $g=g_{2} g_{1}$ is an element of $Q_{[x]}^{\prime \prime}$ such that $\operatorname{ad}(g) Q_{[x]}^{\prime \prime}=Q_{[x]}^{\prime}$. Thus $Q_{[x]}^{\prime \prime}=Q_{[x]}^{\prime}$. It follows that $Q_{[x], 0}^{\prime \prime}=Q_{[x], 0}^{\prime}$ and $Q_{[x], 0}^{\prime \prime}(x)=Q_{[x], 0}^{\prime}(x)$. Q.E.D.

We note the relations of holomorphic and algebraic arc components with projections of flag manifolds.

8.14. Proposition. Let $P_{1} \subset P_{2}$ be parabolic subgroups of $G, X_{i}=G / P_{i}$ the resulting complex flag manifolds, and $\pi: X_{1} \rightarrow X_{2}$ the projection $g P_{1} \rightarrow g P_{2}$. Let $x_{2} \in X_{2}$ and let $G_{0}$ be a real form of $G$.

1. There exist points $x_{1} \in \pi^{-1}\left(x_{2}\right)$ such that $G_{0}\left(x_{1}\right)$ is an open subset of $\pi^{-1} G_{0}\left(x_{2}\right)$.

2. If $x_{1} \in \pi^{-1}\left(x_{2}\right)$, then the following conditions are equivalent:

(2a) $G_{0}\left(x_{1}\right)$ is open in $\pi^{-1} G_{0}\left(x_{2}\right)$.

(2b) $\left(P_{1}\right)_{x_{1}}+\tau\left(P_{1}\right)_{x_{1}}=\left(P_{2}\right)_{x_{2}}+\tau\left(P_{2}\right)_{x_{2}}$.

(2c) codimension $_{R}\left(G_{0}\left(x_{1}\right) \subset X_{1}\right)=$ codimension $_{R}\left(G_{0}\left(x_{2}\right) \subset X_{2}\right)$.

(2d) Let $\mathfrak{H C}\left(\odot_{1}\right)_{x_{1}}$ be a $\tau$-stable Cartan subalgebra of $\mathcal{S}_{1} \Phi_{1} \subset \Phi_{2}$

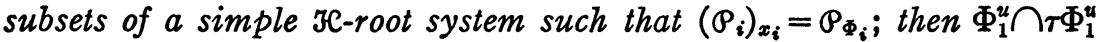
$=\Phi_{2}^{u} \cap \tau \Phi_{2}^{u}$.

3. Let $x_{1} \in \pi^{-1}\left(x_{2}\right)$ with $G_{0}\left(x_{1}\right)$ open in $\pi^{-1} G_{0}\left(x_{2}\right)$. Choose Fe and $\Phi_{1} \subset \Phi_{2}$ as in (2d). Then

(3a) the linear forms $\delta_{\Phi_{1}}=\delta_{\Phi_{2}}$, the parabolic subalgebras $Q_{\left[x_{1}\right]}$ $=Q_{\left[x_{2}\right]}$, and the algebraic arc components of the $G_{0}\left(x_{i}\right)$ are related by

$$
Q_{\left[x_{2}\right], 0}\left(x_{2}\right)=\pi Q_{\left[x_{1}\right], 0}\left(x_{1}\right) \quad \text { and } \quad Q_{\left[x_{1}\right], 0}\left(x_{1}\right)=G_{0}\left(x_{1}\right) \cap \pi^{-1} Q_{\left[x_{2}\right], 0}\left(x_{2}\right) ;
$$

(3b) the spaces $\mathfrak{M C}_{\left[x_{1}\right]}=\mathfrak{M C}_{\left[x_{2}\right]}$, and in particular one is an algebra if and only if the other is an algebra;

(3c) the holomorphic arc components of $G_{0}\left(x_{1}\right)$ are complex submanifolds of $X_{1}$ if and only if the holomorphic arc components of $G_{0}\left(x_{2}\right)$ are complex submanifolds of $X_{2}$;

(3d) holomorphic arc components $S_{\left[x_{i}\right]} \subset G_{0}\left(x_{i}\right) \subset X_{i}$ are related by 


$$
S_{\left[x_{2}\right]}=\pi S_{\left[x_{1}\right]} \text { and } S_{\left[x_{1}\right]}=G_{0}\left(x_{1}\right) \cap \pi^{-1} S_{\left[x_{2}\right]}
$$

(3e) the algebras $\Re_{\left[x_{1}\right]}=\mathscr{\Re}_{\left[x_{2}\right]}$ and the groups $N_{\left[x_{1}\right], 0}=N_{\left[x_{2}\right], 0}$.

Proof. The projection $\pi: X_{1} \rightarrow X_{2}$ is $G_{0}$-invariant, so $\pi^{-1} G_{0}\left(x_{2}\right)$ is a union of $G_{0}$-orbits. As there are only finitely many $G_{0}$-orbits on $X_{1}$, now $\pi^{-1} G_{0}\left(x_{2}\right)$ is a finite union of $G_{0}$-orbits, so at least one of them has full dimension in $\pi^{-1} G_{0}\left(x_{2}\right)$. That proves (1).

If $G_{0}\left(x_{1}\right)$ is open in $\pi^{-1} G_{0}\left(x_{2}\right)$, then

$$
\begin{aligned}
\operatorname{codimension}_{R}\left(G_{0}\left(x_{1}\right) \subset X_{1}\right)= & \operatorname{dim}_{R} X_{1}-\operatorname{dim}_{R} G_{0}\left(x_{1}\right) \\
= & \operatorname{dim}_{R} X_{1}-\operatorname{dim}_{R} \pi^{-1} G_{0}\left(x_{2}\right) \\
= & {\left[\operatorname{dim}_{R} X_{2}+\left\{\operatorname{dim}_{R} X_{1}-\operatorname{dim}_{R} X_{2}\right\}\right] } \\
& -\left[\operatorname{dim}_{R} G_{0}\left(x_{2}\right)+\left\{\operatorname{dim}_{R} X_{1}-\operatorname{dim}_{R} X_{2}\right\}\right] \\
= & \operatorname{dim}_{R} X_{2}-\operatorname{dim}_{R} G_{0}\left(x_{2}\right) \\
= & \operatorname{codimension}_{R}\left(G_{0}\left(x_{2}\right) \subset X_{2}\right) .
\end{aligned}
$$

Conversely, if those codimensions are equal, the argument shows that $\operatorname{dim}_{R} G_{0}\left(x_{1}\right)=\operatorname{dim}_{R} \pi^{-1} G_{0}\left(x_{2}\right)$, so $G_{0}\left(x_{1}\right)$ is open in $\pi^{-1} G_{0}\left(x_{2}\right)$. Thus (2a) and (2c) are equivalent. Theorem 2.12 (ii) says that (2c) is equivalent to $\left|\Phi_{1}^{u} \cap \tau \Phi_{1}^{u}\right|=\left|\Phi_{2}^{u} \cap \tau \Phi_{2}^{u}\right|$. As $\Phi_{2}^{u} \subset \Phi_{1}^{u}$, the latter is equivalent to $\Phi_{1}^{u} \cap \tau \Phi_{1}^{u}=\Phi_{2}^{u} \cap \tau \Phi_{2}^{u}$. Thus (2c) and (2d) are equivalent. We reformulate (2d) as $-\Phi_{1}^{u} \cap-\tau \Phi_{1}^{u}=-\Phi_{2}^{u} \cap-\tau \Phi_{2}^{u}$, i.e. as $\left(P_{1}\right)_{x_{1}}^{-u} \cap \tau\left(P_{1}\right)_{x_{1}}^{-u}$ $=\left(P_{2}\right)_{x_{2}}^{-u} \cap \tau\left(P_{2}\right)_{x_{2}}^{-u}$, i.e. as $\left(P_{1}\right)_{x_{1}}+\tau\left(P_{1}\right)_{x_{1}}=\left(P_{2}\right)_{x_{2}}+\tau\left(P_{2}\right)_{x_{2}}$, which is (2b). That completes the proof of (2).

Let $x_{1} \in \pi^{-1}\left(x_{2}\right)$ with $G_{0}\left(x_{1}\right)$ open in $\pi^{-1} G_{0}\left(x_{2}\right)$. Then we have (2b), (2c) and (2d). From (2d), $\delta_{\Phi_{1}}=\delta_{\Phi_{2}}$, so $Q_{\left[x_{1}\right]}=Q_{\left[x_{2}\right]}, Q_{\left[x_{1}\right], 0}=Q_{\left[x_{2}\right], 0}$ and $Q_{\left[x_{2}\right], 0}\left(x_{2}\right)=\pi Q_{\left[x_{1}\right], 0}\left(x_{1}\right)$; and $\mathcal{P}_{x_{1}} \cap \mathcal{g}_{0} \subset Q_{\left[x_{1}\right], 0}$ implies further that $Q_{\left[x_{1}\right], 0}\left(x_{1}\right)=G_{0}\left(x_{1}\right) \cap \pi^{-1} Q_{\left[x_{2}\right], 0}\left(x_{2}\right)$, completing the proof of (3a). $Q_{\left[x_{1}\right]}=Q_{\left[x_{2}\right]}$ together with (2b) implies $\mathfrak{T}_{\left[x_{1}\right]}=\mathfrak{T}_{\left[x_{2}\right]}$, proving (3b). Theorem 8.9 and (3b) imply (3c). If the holomorphic arc components are complex manifolds then ( $3 \mathrm{~d}$ ) follows from (3b) and Theorem 8.9. But in general we must look at holomorphic arcs.

If $f: D \rightarrow X_{1}$ is a holomorphic arc in $G_{0}\left(x_{1}\right)$ then $(\pi \cdot f): D \rightarrow X_{2}$ is a holomorphic arc in $\pi G_{0}\left(x_{1}\right)=G_{0}\left(x_{2}\right)$. Thus $\pi S_{\left[x_{1}\right]} \subset S_{\left[x_{2}\right]}$. Let $S=G_{0}\left(x_{1}\right)$ $\cap \pi^{-1} S_{\left[x_{2}\right]}, p=\pi \mid s$, and $\xi$ the fibration $p: S \rightarrow S_{\left[x_{2}\right]}$. Then $\xi$ is locally trivial and its fibres carry complex manifold structures as open subsets of $\pi$-fibres. If $y \in S$ this implies $S_{[y]}=p^{-1} p S_{[y]}$. Thus $S_{\left[x_{2}\right]}$ is a disjoint union of sets $p S_{[y]}=\pi S_{[y]}, y \in S$, and $S$ is disjoint union of the corresponding $p^{-1} \pi S_{[y]}$. As the images of holomorphic arcs in $G_{0}\left(x_{2}\right)$ fill out a neighborhood in $S_{\left[x_{2}\right]}$ of every point $p(y) \in S_{\left[x_{2}\right]}$, and as those holomorphic arcs lift to $S$, the sets $p^{-1} \pi S_{[y]}$ are open in $S$, so 
$S=p^{-1} \pi S_{\left[x_{1}\right]}$ by connectedness. That proves (3d). It follows that $\mathfrak{N}_{\left[x_{1}\right], 0} \equiv \mathfrak{N}_{\left[x_{2}\right], 0}$ modulo the isotropy subalgebras of $\mathcal{g}_{0}$ at $x_{1}$ and $x_{2}$. As those isotropy subalgebras $\left(\mathbb{P}_{i}\right)_{x_{i}} \cap \mathcal{G}_{0}=\left(\mathcal{P}_{i}\right)_{x_{i}} \cap \tau\left(P_{i}\right)_{x_{i}} \cap \mathcal{G}_{0} \subset Q_{\left[x_{i}\right]}$ $\cap \mathcal{G}_{0} \subset \mathfrak{N}_{\left[x_{i}\right], 0}$, now $\mathfrak{⿰}_{\left[x_{1}\right], 0}=\mathfrak{N}_{\left[x_{2}\right], 0} ;(3 \mathrm{e})$ follows. Q.E.D.

We describe the "space of holomorphic arc components" of a $G_{0^{-}}$ orbit on $X$.

8.15. Theorem. Let $X=G / P$ be a complex flag manifold, $x \in X, G_{0} a$ real form of $G$, and $K$ a maximal compact subgroup of $G_{0}$. Define projections

$$
\begin{array}{lll}
(8.16 \mathrm{a}) & \sigma: G_{0}(x) \rightarrow G_{0} /\left(G_{0} \cap N_{[x]}\right) & \text { by } \sigma(g x)=g\left(G_{0} \cap N_{[x]}\right), \\
(8.16 \mathrm{~b}) & \kappa: G_{0}(x) \rightarrow G_{0} /\left(G_{0} \cap Q_{[x]}\right) & \text { by } \kappa(g x)=g\left(G_{0} \cap Q_{[x]}\right),
\end{array}
$$

and

$$
\begin{aligned}
\nu: G_{0} /\left(G_{0} \cap Q_{[x]}\right) \rightarrow G_{0} /\left(G_{0} \cap\right. & \left.N_{[x]}\right) \\
& \text { by } g\left(G_{0} \cap Q_{[x]}\right) \rightarrow g\left(G_{0} \cap N_{[x]}\right) .
\end{aligned}
$$

1. $\sigma$ is well defined and (8.16a) is a $G_{0}$-equivariant differentiable fibre bundle with

(1a) structure group $G_{0} \cap N_{[x]}$, the parabolic subgroup of $G_{0}$ with identity component $N_{[x], 0}$,

(1b) fibre over $\sigma(g x)$ equal to the holomorphic arc component $S_{[g x]}$ $=g S_{[x]}$ of $G_{0}(x)$ through $g(x)$, and

(1c) compact base $G_{0} /\left(G_{0} \cap N_{[x]}\right)=K /\left(K \cap N_{[x]}\right)$, real fag manifold that parameterizes the holomorphic arc components of $G_{0}(x)$.

2. $\mathrm{\kappa}$ is well defined and (8.16b) is a $G_{0}$-equivariant differentiable fibre bundle with

(2a) structure group $G_{0} \cap Q_{[x]}$, the parabolic subgroup of $G_{0}$ with identity component $Q_{[x], 0}$,

(2b) fibre over $\kappa(g x)$ equal to the algebraic arc component $Q_{[g x], 0}(g x)$ $=g Q_{[x], 0}(x)$ of $G_{0}(x)$ through $g(x)$, and

(2c) compact base $G_{0} /\left(G_{0} \cap Q_{[x]}\right)=K /\left(K \cap Q_{[x]}\right)$, real flag manifold that parameterizes the algebraic arc components of $G_{0}(x)$.

3. $\nu=\sigma \cdot \kappa^{-1}$, well-defined projection of real flag manifolds, and (8.16c) has fibre over $\nu(g x)$ parameterizing the algebraic arc components of $G_{0}(x)$ that are contained in the holomorphic arc component $S_{[g x]}$.

Proof. $\rho_{x} \cap \tau \mathcal{P}_{x} \subset Q_{[x]} \subset \mathscr{N}_{[x]}$ implies $G_{0} \cap P_{x} \subset G_{0} \cap Q_{[x]} \subset G_{0} \cap N_{[x]}$, so $\sigma, \kappa$ and $\nu=\sigma \cdot \kappa^{-1}$ are well defined, and $(8.16 \mathrm{a}, \mathrm{b}, \mathrm{c})$ are $G_{0}$-equivariant differentiable fibre bundles with structure groups as described in (1a) and (2a).

Let $F$ be the complex flag manifold $G / N_{[x]}$ or $G / Q_{[x]}$. View $G$ as a 
linear algebraic group def/R such that $G_{0}$ is the topological identity component of the set $G_{\mathrm{R}}$ of real points. Then $F$ is def/R and Theorem 3.6(2) says that the $G_{0}$-orbit of the identity coset on $F$ is $F_{\mathrm{R}}$, and that it is the unique closed $G_{0}$-orbit on $F$. That proves that the base spaces of (8.16a) and (8.16b) are real flag manifolds and that $K$ acts transitively on them. Now it only remains to prove (1b) and (2b); for then (1c), (2c) and (3c) will follow immediately.

Choose a $\tau$-stable Cartan subalgebra $\mathfrak{H C} \subset \boldsymbol{P}_{x}$ of $\mathcal{G}$ and decompose $\mathfrak{H C}_{0}=\mathfrak{H C}_{\mathcal{C}} \cap \mathcal{G}_{0}$ as $\mathfrak{H C}_{T}+\mathfrak{H C}_{V}$, toroidal and vector parts relative to a Cartan involution $\theta$ of $\mathcal{G}_{0}$. The choice of $\Re$ determines reductive parts $\mathcal{P}_{x}^{r}$, $Q_{[x]}^{r}$ and $\Re_{[x]}^{r}$; let $Z_{P} \subset \mathcal{F}, Z_{Q} \subset \mathcal{H}$ and $z_{N} \subset \mathcal{H C}$ denote their respective centers, and let $Z \subset \mathcal{K C}$ denote the center of ${P_{x}^{r}}_{x} \cap{P_{x}^{r}}_{x}$; note $\tau Z=Z$, $\tau \mathrm{Z}_{Q}=\mathrm{Z}_{Q}, \tau \mathrm{Z}_{N}=\mathrm{Z}_{N}$ and $\mathrm{Z}_{N} \subset \mathrm{Z}_{Q} \subset \mathrm{Z}$.

$\mathfrak{T}_{[x]}^{r}$ is the centralizer of $Z_{N}$ in $\mathcal{G}, Q_{[x]}^{r}$ is the centralizer of $Z_{Q}$ in $\mathcal{G}$, and $P_{x}^{r}$ is the centralizer of $Z_{P}$ in $\mathcal{G}$, because $\mathscr{T}_{[x]}, \mathcal{Q}_{[x]}$ and $\rho_{x}$ are parabolic subalgebras of $\mathrm{G}$. Now $\rho_{x}^{r} \cap \tau \mathcal{P}_{x}^{r}$ is the centralizer of $Z_{P}+\tau Z_{P}$ in $\mathcal{G}$, and it follows that $\boldsymbol{P}_{x}^{r} \cap \tau \mathcal{P}_{x}^{r}$ is the centralizer of $\mathcal{Z}$ in $\mathcal{G}$. Decompose real forms:

$$
\begin{aligned}
& Z_{N, 0}=Z_{N} \cap g_{0} \text { and } Z_{N, 0}=Z_{N, T}+Z_{N, V} \text {, } \\
& Z_{Q, 0}=Z_{Q} \cap g_{0} \text { and } z_{Q, 0}=Z_{Q, T}+Z_{Q, V} \text {, } \\
& \mathrm{Z}_{0}=\mathrm{Z} \cap \mathrm{g}_{0} \text { and } \mathrm{Z}_{0}=\mathrm{Z}_{T}+\mathrm{Z}_{\mathrm{V}}
\end{aligned}
$$

into toroidal and vector parts by intersecting with $\mathfrak{H C}_{T}$ and $\mathfrak{H C}_{\nabla}$. If $s \subset g$ then $Z(\delta)$ denotes the centralizer of $\delta$ in $G$ and $Z_{0}(\delta)$ denotes the centralizer $G_{0} \cap Z(\mathcal{S})$ of $S$ in $G_{0}$. Now we have $N_{[x]}^{r}=Z\left(Z_{N, T}\right)$ $\cap Z\left(Z_{N, v}\right), \quad Q_{[x]}^{r}=Z\left(Z_{Q, T}\right) \cap Z\left(Z_{Q, v}\right)$ and $P_{x}^{r} \cap \tau P_{x}^{r}=Z\left(\mathrm{Z}_{T}\right) \cap Z\left(\mathrm{Z}_{V}\right)$; for $Z(S)=Z\left(S \cap \mathcal{G}_{0}\right)$ whenever $S$ is a $\tau$-stable subspace of $\mathcal{G}$. Thus

$$
\begin{aligned}
& G_{0} \cap N_{[x]}^{r}=Z_{0}\left(Z_{N, T}\right) \cap Z_{0}\left(Z_{N, V}\right), \\
& G_{0} \cap Q_{[x]}^{r}=Z_{0}\left(Z_{Q, T}\right) \cap Z_{0}\left(Z_{Q, V}\right),
\end{aligned}
$$

and

$$
G_{0} \cap P_{x}^{r} \cap \tau P_{x}^{r}=Z_{0}\left(Z_{T}\right) \cap Z_{0}\left(Z_{V}\right) .
$$

It follows that

$$
\begin{aligned}
& G_{0} \cap \exp \left(i Z_{N, V}\right) \text { meets every component of } G_{0} \cap N_{[x]}^{r}, \\
& G_{0} \cap \exp \left(i Z_{Q, V}\right) \text { meets every component of } G_{0} \cap Q_{[x]}^{r},
\end{aligned}
$$

and

$$
G_{0} \cap \exp \left(i \mathcal{Z}_{V}\right) \text { meets every component of } G_{0} \cap P_{x}^{r} \cap \tau P_{x}^{r} .
$$




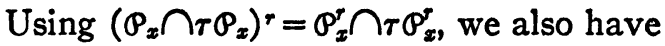

$G_{0} \cap \exp \left(i \mathcal{Z}_{V}\right)$ meets every component of $G_{0} \cap\left(P_{x} \cap \tau P_{x}\right)^{r}$.

Connectivity of real unipotent groups says that $G_{0} \cap N_{[x]}^{r}$ meets every component of $G_{0} \cap N_{[x]}, G_{0} \cap Q_{[x]}^{r}$ meets every component of $G_{0} \cap Q_{[x]}$, and $G_{0} \cap\left(P_{x} \cap \tau P_{x}\right)^{r}$ meets every component of $G_{0} \cap\left(P_{x} \cap \tau P_{x}\right)$ $=G_{0} \cap P_{x}$. Thus

$G_{0} \cap \exp \left(i Z_{N, V}\right)$ meets every component of $G_{0} \cap N_{[x]}$,

$G_{0} \cap \exp \left(i Z_{Q, V}\right)$ meets every component of $G_{0} \cap Q_{[x]}$,

and

$G_{0} \cap \exp \left(i Z_{V}\right)$ meets every component of $G_{0} \cap P_{x}$.

$\mathrm{Z}_{N} \subset \mathrm{Z}_{Q} \subset \mathrm{Z}$ implies $i \mathrm{Z}_{N, V} \subset i \mathrm{Z}_{Q, V} \subset i \mathrm{Z}_{V}$, so $G_{0} \cap \exp \left(i \mathrm{Z}_{N, V}\right) \subset G_{0}$ $\cap \exp \left(i Z_{Q, V}\right) \subset G_{0} \cap \exp \left(i Z_{V}\right)$. Thus $G_{0} \cap P_{x}$ meets every component of $G_{0} \cap Q_{[x]}$ and also meets every component of $G_{0} \cap N_{[x]}$. This proves that $\left(G_{0} \cap Q_{[x]}\right)(x)$ and $\left(G_{0} \cap N_{[x]}\right)(x)$ are connected, hence equal to $Q_{[x], 0}(x)$ and $N_{[x], 0}(x)=S_{[x]}$, respectively. That proves (1b) and (2b), completing the proof of the theorem. Q.E.D.

8.17. Definitions. In the notation of Theorem 8.15 , the base space $G_{0} /\left(G_{0} \cap N_{[x]}\right)$ of the fibre bundle (8.16a) will be called the space of holomorphic arc components of $G_{0}(x)$, and the base $G_{0} /\left(G_{0} \cap Q_{[x]}\right)$ of the bundle (8.16b) will be called the space of algebraic arc components of $G_{0}(x)$. This terminology is based on results (1c) and (2c) of Theorem 8.15.

8.18. Corollary. Let $P_{1} \subset P_{2}$ be parabolic subgroups of $G, X_{i}=G / P_{i}$ the resulting complex flag manifolds, $\pi: X_{1} \rightarrow X_{2}$ the projection $g P_{1} \mapsto g P_{2}$, $G_{0}$ a real form of $G, x_{2} \in X_{2}$, and $x_{1} \in \pi^{-1}\left(x_{2}\right)$ such that $G_{0}\left(x_{1}\right)$ is open in $\pi^{-1} G_{0}\left(x_{2}\right)$.

1. $\pi$ induces a diffeomorphism $S_{[x]} \mapsto \pi S_{[x]}=S_{[\pi x]}$ from the space of holomorphic arc components of $G_{0}\left(x_{1}\right)$ onto the space of holomorphic arc components of $G_{0}\left(x_{2}\right)$.

2. $\pi$ induces a diffeomorphism $Q_{[x], 0}(x) \mapsto \pi Q_{[x], 0}(x)=Q_{[\pi x], 0}(\pi x)$ from the space of algebraic arc components of $G_{0}\left(x_{1}\right)$ onto the space of algebraic arc components of $G_{0}\left(x_{2}\right)$.

Proof. This is immediate from Theorem 8.15 and the results $Q_{\left[x_{1}\right]}=Q_{\left[x_{2}\right]}$ and $N_{\left[x_{1}\right]}=N_{\left[x_{2}\right]}$ of Proposition 8.14(3). Q.E.D.

8.19. Corollary. Let $X=G / P$ be a complex flag manifold, $x \in X$, and $G_{0}$ a real form of $G$. Then the following conditions are equivalent:

(i) $G_{0}(x)$ is the unique closed $G_{0}$-orbit on $X$.

(ii) $G_{0}(x)$ is compact. 
(iii) The holomorphic arc components of $G_{0}(x)$ are compact.

(iv) The algebraic arc components of $G_{0}(x)$ are compact.

Proof. We have noted equivalence of (i) and (ii) from compactness of $X$. Now consider the fibrations (8.16)

$$
\begin{aligned}
& \sigma: G_{0}(x) \rightarrow G_{0} /\left(G_{0} \cap N_{[x]}\right), \\
& \kappa: G_{0}(x) \rightarrow G_{0} /\left(G_{0} \cap Q_{[x]}\right)
\end{aligned}
$$

whose base spaces, both compact, are the respective spaces of holomorphic and algebraic arc components of $G_{0}(x)$. As the base is compact, the total space is compact if and only if the fibres are compact. That given equivalence of (ii) with (iii) and with (iv). Q.E.D.

8.20. Corollary. Let $X=G / P$ be a complex flag manifold, $G_{0}$ a real form of $G$, and $x \in X$. If the holomorphic arc components of $G_{0}(x)$ are points, then $G_{0}(x)$ is the closed $G_{0}$-orbit on $X$. Semiconversely, if $G_{0}(x)$ is the closed $G_{0}$-orbit on $X$, and if $\operatorname{dim}_{R} G_{0}(x)=\operatorname{dim}_{C} X$, then the holomorphic arc components of $G_{0}(x)$ are points.

Proof. The first assertion follows from Corollary 8.19 because points are compact. Now suppose $\operatorname{dim}_{R} G_{0}(x)=\operatorname{dim}_{C} X$. Then $\mathcal{P}_{x}=\tau \mathcal{P}_{x}$ by Theorem 3.6, so $\mathcal{P}_{x} \cap \tau \mathcal{P}_{x}=\mathcal{P}_{x}+\tau \mathcal{P}_{x}$, and the construction (8.8) yields $\mathfrak{T}_{[x]}$ as the algebra $\odot_{x}$. Theorem 8.9 then says $\mathfrak{T}_{[x]}=\odot_{x}$, so $S_{[x]}=N_{[x], 0}(x)=\left(G_{0} \cap P_{x}\right)(x)=x$. Q.E.D.

9. Global conditions for the components of an orbit. We will study certain conditions related to complexity and measurability for the holomorphic arc components of a $G_{0}$-orbit. Those conditions are defined as follows.

9.1. Definitions. Let $X=G / P$ be a complex flag manifold, $G_{0}$ a real form of $G, x \in X, S_{[x]}$ the holomorphic arc component of $G_{0}(x)$ through $x$, $N_{[x], 0}$ the identity component of the normalizer of $S_{[x]}$ in $G_{0}$, and $N_{[x]}$ $=N_{[x], 0}^{C}$.

1. The orbit $G_{0}(x)$ is called partially complex if its holomorphic arc components are complex submanifolds of $X$, i.e. if $S_{[x]}$ is a complex submanifold of $X$.

2. The orbit $G_{0}(x)$ is said to be of flag type if the $N_{\left[x^{\prime}\right]}\left(x^{\prime}\right), x^{\prime} \in G_{0}(x)$, are complex flag manifolds, i.e. if $N_{[x]}(x)$ is a complex flag manifold, i.e. if $N_{[x]}$ has a $\tau$-stable reductive part $N_{[x]}^{r}$ that is transitive on $N_{[x]}(x)$ and that has $N_{[x]}^{r} \cap P_{x}$ as a parabolic subgroup.

3. The orbit $G_{0}(x)$ is called measurable if its holomorphic arc components carry invariant positive Radon measures, i.e. if $S_{[x]}$ carries an $N_{[x], 0-i n v a r i a n t}$ positive Radon measure. 
4. The orbit $G_{0}(x)$ is said to be polarized if the $P_{x^{\prime}}, x^{\prime} \in G_{0}(x)$, have $\tau$-stable reductive parts, i.e. if $\tau \odot_{x}^{\gamma}=P_{x}^{r}$ for some choice of reductive part $\mathcal{P}_{x}^{r} \subset \mathcal{P}_{x}$.

5. The orbit $G_{0}(x)$ is called integrable if the distribution $T$ of (8.10) is integrable, i.e. if $P_{x}+\tau P_{x}$ is a subalgebra of $\mathrm{G}$.

Theorem 8.9 gives several conditions for $G_{0}(x)$ to be partially complex. The analogous condition for algebraic arc components is automatic (Lemma 8.13), so it will not be given a name.

The notion of flag type is a useful hereditary condition. The analogous condition for algebraic arc components is that $Q_{[x]}(x)$ be a complex flag manifold. In that case we will say that $G_{0}(x)$ is of flag type relative to its algebraic arc components.

The notion of measurable orbit is the primary object of this paper. In $\$ 6$ we obtained the basic facts for measurable open orbits, and the definition here specializes to that of $\$ 6$. The analogous condition for algebraic arc components is that $Q_{[x], 0}(x)$ carry a $Q_{[x], 0 \text {-invariant }}$ positive Radon measure. In that case we will say that $G_{0}(x)$ is measurable relative to its algebraic arc components.

The notion of polarized orbit will be useful in studying the three concepts above. Let $\mathfrak{H} \subset \boldsymbol{P}_{x}$ be any $\tau$-stable Cartan subalgebra of $\mathcal{G}$. That determines a choice of $\odot_{x}^{r}$, and Lemma 2.10 says $\left(\odot_{x} \cap \tau \odot_{x}\right)^{r}$ $=\mathcal{P}_{x}^{r} \cap \tau \mathcal{P}_{x}^{r}$. Thus $G_{0}(x)$ is polarized if and only if $\operatorname{dim}\left(\mathcal{P}_{x} \cap \tau \odot_{x}\right)^{r}$ $=\operatorname{dim}{\mathcal{P}_{x}^{r}}^{r}$. The latter can be expressed as $\operatorname{dim}\left\{\left(\mathcal{P}_{x} \cap \tau \mathcal{P}_{x}\right) /\left(\mathcal{\odot}_{x} \cap \tau \mathcal{P}_{x}\right)^{u}\right\}$ $=\operatorname{dim}\left\{\odot_{x} / \mathcal{P}_{x}^{u}\right\}$, which is independent of choice of $\mathcal{H}$. Thus $G_{0}(x)$ is polarized if and only if $P_{x}^{r}=\tau P_{x}^{r}$ relative to every $\tau$-stable Cartan subalgebra $\mathfrak{H C} \mathcal{P}_{x}$ of $\mathcal{G}$.

The notion of integrable orbit is recalled from $\S 7$, where it was studied in some detail.

9.2. Theorem. Let $X=G / P$ be a complex flag manifold, $G_{0}$ a real form of $G, x \in X, \mathfrak{H} \subset \mathcal{P}_{x}$ a $\tau$-stable Cartan subalgebra of $\mathcal{G}$, and $\Phi$ a subset of a simple HC-root system such that $\rho_{x}=\odot_{\Phi}$. Define

$$
V_{x}^{+}=\sum_{\Phi^{u} \cap-\tau \Phi^{u}} S_{\varphi}, \quad V_{x}^{-}=\tau V_{x}^{+}=\sum_{-\Phi^{u} n_{\tau \Phi^{u}}} S_{\varphi}, \quad V_{x}=V_{x}^{+}+V_{x}^{-} .
$$

Then $G_{0}(x)$ is measurable if and only if

$$
\begin{gathered}
\mathscr{T}_{[x]}^{u}=\left(\odot_{x} \cap \tau \odot_{x}\right)^{u}, \quad \Re_{[x]}^{r}=\left(\odot_{x} \cap \tau \odot_{x}\right)^{r}+V_{x}, \\
\mathscr{N}_{[x]}=\left(\odot_{x} \cap \tau \odot_{x}\right)+V_{x} .
\end{gathered}
$$

If $G_{0}(x)$ is measurable, then

(i) $G_{0}(x)$ is partially complex;

(ii) $G_{0}(x)$ is of flag type; 
(iii) $G_{0}(x)$ is polarized if and only if it is integrable; and

(iv) the invariant Radon measure on $S_{[x]}$ is the volume element of an

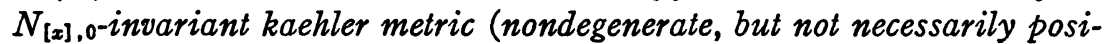
tive definite).

PRoof. Let $A$ be the set of $\mathcal{F}$-roots such that $\mathscr{T}_{[x]}=\left(\odot_{x} \cap \tau \odot_{x}\right)$ $+\sum_{A} g_{\varphi}$ and define $\alpha=\sum_{A} \varphi$. If $G_{0}(x)$ is measurable, then $S_{[x]}$ has

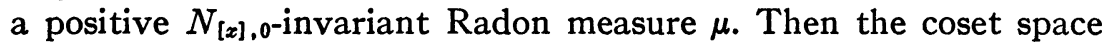
$N_{[x], 0} /\left(N_{[x], 0} \cap P_{x}\right)=N_{[x], 0} /\left(P_{x} \cap \tau P_{x}\right)_{0}$ representing $S_{[x]}$ has $\mu$ as invariant measure, so $\left(P_{x} \cap P_{x}\right)_{0}$ acts with determinant \pm 1 on its tangent space, i.e. $\left(\mathcal{P}_{x} \cap \tau \mathcal{P}_{x}\right)_{0}$ acts with trace 0 on $\mathfrak{T}_{[x], 0} /\left(\mathcal{P}_{x} \cap \tau \mathcal{P}_{x}\right)_{0}$, i.e. $\mathcal{P}_{x} \cap \tau \odot_{x}$ acts with trace 0 on $\mathcal{T}_{[x]} /\left(\mathcal{P}_{x} \cap \tau \mathcal{P}_{x}\right)$, i.e. $\left(\mathcal{P}_{x} \cap \tau \odot_{x}\right)^{r}$ acts with trace 0 on $\sum_{A} \oint_{\varphi}$, i.e. $\mathcal{F}$ acts with trace 0 on $\sum_{A} \oint_{\varphi}$, i.e. $\alpha=0$. The argument is reversible. Thus $G_{0}(x)$ is measurable if and only if $\alpha=0$.

Suppose $G_{0}(x)$ measurable. If $\varphi \in A$ with $g_{\varphi} \subset \Re_{[x]}^{u}$, then $\alpha$ has positive inner product with the sum of the roots of $\mathscr{T}_{[x]}^{u}$, contradicting $\alpha=0$. Thus $\sum_{A} \oint_{\varphi} \subset \Re_{[x]}^{\tau}$ and it follows that $\Re_{[x]}^{u} \subset\left(\rho_{x} \cap \tau \odot_{x}\right)$. Now $A=-A$ by Lemma 6.2 , for $\mathfrak{N}_{[x]}^{\tau} \cap\left(\odot_{x} \cap \tau \odot_{x}\right)$ is reductive. As $A$ is disjoint from $\Phi^{*} \cap \tau \Phi^{*}$, i.e. as

$A \subset\left(\Phi^{r} \cap-\tau \Phi^{u}\right) \cup\left(-\Phi^{u} \cap \tau \Phi^{r}\right) \cup\left(\Phi^{u} \cap-\tau \Phi^{u}\right) \cup\left(-\Phi^{u} \cap \tau \Phi^{u}\right)$,

this says $\sum_{A} \mathcal{S}_{\varphi} \subset \mathcal{V}_{x}$. As $\mathscr{T}_{[x]}$ is parabolic now $\sum_{A} \mathcal{S}_{\varphi}=V_{x}$. Thus $\mathfrak{N}_{[x]}=\left(\odot_{x} \cap \tau \odot_{x}\right)+\mathcal{V}_{x}$. That proves (9.4).

Conversely assume (9.4). Then $N_{[x], 0}^{u}$ acts trivially on $S_{[x]}$ and $N_{[x], 0}^{r}$ acts transitively with reductive isotropy subgroup $\left(P_{x}^{r} \cap \tau P_{z}^{r}\right)_{0}$, so Lemma 6.2 says that $G_{0}(x)$ is measurable.

Suppose $G_{0}(x)$ measurable. Then (9.4) implies $\Re_{[x]} \subset\left(\mathcal{P}_{x}+\tau \mathcal{P}_{x}\right)$, so Theorem 8.9 says that $G_{0}(x)$ is partially complex; that proves (i). $\mathfrak{N}_{[x]}^{u} \subset \mathcal{P}_{x}$ by (9.4), so $N_{[x]}^{u}$ acts trivially on $N_{[x]}(x)$, whence $N_{[x]}^{r}$ is transitive on $N_{[x]}(x)$; the isotropy subalgebra of $\Re_{[x]}^{r}$ at $x$ is $\mathfrak{T}_{[x]}^{\tau} \cap \boldsymbol{P}_{x}$ $=\left(\mathcal{P}_{x} \cap \tau \mathcal{P}_{x}\right)^{r}+v_{x}^{+}$, parabolic subalgebra; thus $N_{[x]}(x)$ is a complex flag manifold; that proves (ii). If $G_{0}(x)$ is polarized then $\tau \Phi^{r}=\Phi^{r}$, so $\left(\mathcal{P}_{x} \cap \tau \mathcal{P}_{x}\right)=\mathcal{P}_{x}^{r}+\tau \mathcal{P}_{x}^{r}+\left(\mathcal{P}_{x}^{u} \cap \tau \mathcal{P}_{x}^{u}\right)$ so $\left(\mathcal{P}_{x} \cap \tau \mathcal{P}_{x}\right)+\nu_{x}=\left(\mathcal{P}_{x}+\tau \mathcal{P}_{x}\right)$, and (9.4) says that $\mathscr{\Re}_{[x]}=\left(\mathcal{\odot}_{x}+\tau \odot_{x}\right)$; thus $\mathcal{P}_{x}+\tau \mathcal{P}_{x}$ is an algebra and $G_{0}(x)$ is integrable. If $G_{0}(x)$ is integrable then $\mathscr{T}_{[x]}=\left(\mathcal{P}_{x}+\tau \odot_{x}\right)$ by Theorem 8.9, for $Q_{[x]}=\left(\mathcal{P}_{x} \cap \tau \mathcal{P}_{x}\right)$; then (9.4) says that $\Phi^{r} \cap-\tau \Phi^{u}$ and $-\Phi^{u} \cap \tau \Phi^{r}$ are empty, so $\tau \Phi^{r}=\Phi^{r}$ and $G_{0}(x)$ is polarized. That proves (iii).

Suppose $G_{0}(x)$ measurable. By (ii), $Y=N_{[x]}(x)$ is a complex flag manifold $N_{[x]}^{r} /\left(N_{[x]}^{\tau} \cap P_{x}\right)$ on which $N_{[x]}^{u}$ acts trivially. Lemma 8.4 says that $S_{[x]}=N_{[x, 0}^{r}(x)$ is an open $N_{[x], 0}^{r}$-orbit on $Y$. Now $S_{[x]}$ is a measurable open $N_{[x], 0}^{r} 0^{-}$orbit on the complex flag manifold $Y$, so 
Theorem 6.3 says that its measure is the volume element of a (possibly indefinite) $N_{[x], 0}^{r}$-invariant kaehler metric $d s^{2}$. As $N_{[x], 0}^{u}$ acts trivially on $S_{[x]}$ it must preserve $d s^{2}$. Thus $d s^{2}$ is $N_{[x], 0}$-invariant. That proves (iv), completing the proof of the theorem. Q.E.D.

9.5. Corollary. Let $X=G / P$ be a complex flag manifold, $G_{0}$ a real form of $G, x \in X$, and $v_{x}$ as defined in (9.3). Then $G_{0}(x)$ is measurable relative to its algebraic arc components if and only if

$$
\begin{gathered}
Q_{[x]}^{u}=\left(\odot_{x} \cap \tau \odot_{x}\right)^{u}, \quad Q_{[x]}^{r}=\left(\mathcal{P}_{x} \cap \tau \odot_{x}\right)^{r}+v_{x}, \\
Q_{[x]}=\left(\mathcal{P}_{x} \cap \tau \odot_{x}\right)+v_{x} .
\end{gathered}
$$

In that case,

(i) $G_{0}(x)$ is of flag type relative to its algebraic arc components;

(ii) $G_{0}(x)$ is polarized if and only if it is integrable;

(iii) the invariant Radon measure on $Q_{[x], 0}(x)$ is the volume element of

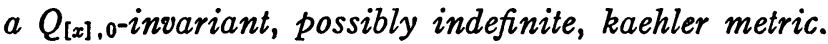

PRoof. $Q_{[x]}$ can be substituted for $\mathfrak{T}_{[x]}$ throughout the proof of Theorem 9.2. Q.E.D.

To decide whether a given $G_{0}(x)$ is measurable, compute $\delta_{\Phi}$ and $Q_{[x]}$ by (8.6), then $\mathfrak{N}_{[x]}$ by (8.8), and finally $\boldsymbol{w}_{[x]}=\left(\mathcal{P}_{x} \cap \tau \mathcal{P}_{x}\right)+\mathcal{V}_{x}$ by (9.3). Then $G_{0}(x)$ is measurable if and only if (i) $\mathfrak{N}_{[x]}=\mathscr{W}_{[x]}$ and (ii) $W_{[x]}$ is an algebra. It is easy to check (i), and it follows from (9.3) that (ii) is equivalent to $\left[v_{x}^{+}, v_{x}^{-}\right] \subset W_{[x]}$. The latter inclusion can be unpleasant to check, but it is much more easily decidable than is the inclusion $\left[\mathfrak{T}_{[x]}, \mathfrak{N}_{[x]}\right] \subset \mathfrak{M}_{[x]}$ that says $\mathfrak{M}_{[x]}$ is an algebra. So measurability is easier to check than is partial complexity.

9.7. THEOREM. Let $X=G / P$ be a complex flag manifold, $G_{0}$ a real form of $G, x \in X, \mathfrak{H C} \subset P_{\mathbf{x}}$ a $\tau$-stable Cartan subalgebra of $\mathcal{G}, \Phi$ a subset of $a$ simple JC-root system such that $\odot_{x}=\odot_{\Phi}$, and $\vartheta_{x}$ as in (9.3).

1. $G_{0}(x)$ is of flag type if and only if $v_{x} \subset \mathfrak{Y}_{[x]}$.

2. If $G_{0}(x)$ is of flag type, then $G_{0}(x)$ is partially complex if and only if $S_{[x]}=N_{[x], 0}^{r}(x)$ is an open $N_{[x], 0}^{r}$ orbit on the complex flag manifold $N_{[x]}^{+}(x)$.

3. If $G_{0}(x)$ is partially complex and of flag type, then $\operatorname{dim}_{c} S_{[x]}$ $\geqq\left|\Phi^{u} \cap-\tau^{u}\right|$, with equality if and only if $G_{0}(x)$ is measurable.

Proof. Suppose $G_{0}(x)$ of flag type, so $N_{|x|}^{r}$ is transitive on $Y=N_{[x]}(x)$ and $N_{[x]}^{\tau} \cap P_{x}$ is parabolic in $N_{[x]}^{r}$. As $N_{[x]}^{u}$ is solvable it has a fixed point $y \in Y$. If $g \in N_{[x]}$ then $N_{[x]}^{u}(g y)=g N_{[x]}^{u}(y)=g y$; thus $N_{[x]}^{u}$ acts trivially on $Y$; now $\mathfrak{T}_{[x]}^{u} \subset \mathcal{P}_{x}$, so $\tau \mathfrak{T}_{[x]}^{u}=\mathfrak{N}_{[x]}^{u}$ gives us $\mathfrak{N}_{[x]}^{u}$ $C\left(\odot_{x} \cap \tau \odot_{x}\right)$. If $\varphi \in \Phi^{u} \cap-\tau \Phi^{u}$, it follows that neither $\varphi$ nor $-\varphi$ is a root of $\Re_{[x]}^{u}$, so both are roots of $\Re_{[x]}^{r}$; thus $v_{x} \subset \Re_{[x]}$. 


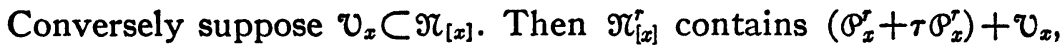
and $\Re_{[x]}$ contains $\left(\mathcal{P}_{x} \cap \tau \mathcal{P}_{x}\right)^{u}$; it follows that $\boldsymbol{T}_{[x]}^{u} \subset\left(\mathcal{P}_{x} \cap \tau \mathcal{P}_{x}\right)^{u}$, so $N_{[x]}^{r}$ is transitive on $N_{[x]}(x)$. The isotropy subalgebra $\mathfrak{N}_{[x]}^{r} \cap \rho_{x}$ contains $\left(\mathcal{P}_{x} \cap \tau \mathcal{P}_{x}\right)+\mathcal{V}_{x}^{+}$, so it is parabolic in $\mathfrak{r}_{[x]}^{\tau}$. Thus $G_{0}(x)$ is of flag type.

Let $G_{0}(x)$ be of flag type, $Y=N_{[x]}(x)=N_{[x]}^{\tau}(x)$. Then $S_{[x]}=N_{[x], 0}(x)$ $=N_{[x], 0}^{t}(x)$. If $G_{0}(x)$ is partially complex, then $\Re_{x} \subset\left(\mathcal{P}_{x}+\tau \odot_{x}\right)$ by Theorem 8.9, so $S_{[x]}$ is open in $Y$ by Lemma 8.4. If $S_{[x]}$ is open in $Y$, then it is a complex submanifold of $X$, so $G_{0}(x)$ is partially complex.

Let $G_{0}(x)$ be partially complex and of flag type. Then $\Re_{[x]}$ $=\left(\mathcal{P}_{x} \cap \tau \mathcal{P}_{x}\right)+v_{x}+w_{x}$ where $w_{x}$ is a $\tau$-stable sum of root spaces $\mathcal{G}_{\varphi}$, $\varphi \in\left(\Phi^{r} \cap-\tau \Phi^{u}\right) \cup\left(-\Phi^{u} \cap \tau \Phi^{r}\right)$. Now $\operatorname{dim}_{C} S_{[x]}=\operatorname{dim}_{C} N_{[x]}(x)$ $=\operatorname{dim}_{C} \mathcal{V}_{x}^{-}+\operatorname{dim}_{C}\left(W_{x} \cap \tau \mathcal{P}_{x}\right)=\left|\Phi^{u} \cap-\tau \Phi^{u}\right|+\operatorname{dim}_{C}\left(W_{x} \cap \tau \mathcal{P}_{x}\right)$ $\geqq\left|\Phi^{u} \cap-\tau \Phi^{u}\right|$, and equality means $\mathfrak{w}_{x}=0$, i.e. $\mathfrak{T}_{[x]}=\left(\mathcal{\odot}_{x} \cap \tau \odot_{x}\right)+V_{x}$, i.e. $G_{0}(x)$ measurable. Q.E.D.

9.8. Corollary. Let $X=G / P$ be a complex flag manifold, $G_{0}$ a real form of $G, x \in X, \mathfrak{x} \subset \odot_{x}$ a $\tau$-stable Cartan subalgebra of $\mathcal{G}, \Phi$ a subset of a simple $\mathfrak{H C}$-root system such that $\mathcal{P}_{x}=\mathcal{P}_{\Phi}$, and $\vartheta_{x}$ as in (9.3).

1. $G_{0}(x)$ is of flag type relative to its algebraic arc components if and only if $v_{x} \subset Q_{[x]}$.

2. If $G_{0}(x)$ is of flag type relative to its algebraic arc components, then (2a) $G_{0}(x)$ is of flag type,

(2b) the algebraic arc component of $G_{0}(x)$ through $x$ is an open $Q_{[x], 0-o r b i t}^{x}$ on the complex flag manifold $Q_{[x]}^{[}(x)$,

(2c) the algebraic arc components of $G_{0}(x)$ have complex dimension $\geqq\left|\Phi^{u} \cap-\tau \Phi^{u}\right|$, with equality if and only if $G_{0}(x)$ is measurable relative to its algebraic arc components.

PRoof. $Q_{[x]}$ can be substituted for $\mathfrak{N}_{[x]}$ throughout the proof of Theorem 9.7. Q.E.D.

Recall Example 8.12. There $G=\mathrm{SL}(2 m, \mathbf{C}), G_{0}$ is $\mathbf{S L}(m, \mathrm{Q})$ or $\operatorname{SL}(2 m, \mathrm{R})$, the simple root system $\Psi=\left\{\psi_{1}, \cdots, \psi_{2 m-1}\right\}, \tau \psi_{i}=\psi_{2 m-i}$, and $\mathcal{P}_{x}=\mathcal{P}_{\Phi}$ where $\Phi=\left\{\psi_{1}, \cdots, \psi_{2 m-2}\right\}$. So $X$ is complex projective $(2 m-1)$-space and we calculated

$Q_{[x]}=\odot_{\Gamma}$ where $\Gamma=\left\{\psi_{2}, \cdots, \psi_{2 m-2}\right\}$ and

$\mathfrak{T}_{[x]}=\boldsymbol{\vartheta}_{x}+\tau \mathcal{P}_{x}=\sum_{\varphi \neq-\mu} \oint_{\varphi}$ where $\mu=\psi_{1}+\psi_{2}+\cdots+\psi_{2 m-1}$.

Thus $\mathfrak{T}_{[x]}$ generates $\mathcal{G}$. As $\mathfrak{N}_{[x]} \subset \mathfrak{N}_{[x]}$ by Proposition 8.7, it follows that $\Re_{[x]}=\mathrm{g}$. Thus $G_{0}(x)$ is of flag type, but it is not partially complex.

9.9. THEOREM. Let $X=G / P$ be a complex flag manifold, $G_{0}$ a real form of $G$, and $x \in X$ such that the orbit $G_{0}(x)$ is polarized. Then the following conditions are equivalent: 
(i) $G_{0}(x)$ is integrable.

(ii) $G_{0}(x)$ is measurable.

(iii) $G_{0}(x)$ is measurable relative to its algebraic arc components.

(iv) $G_{0}(x)$ is partially complex and of flag type.

(v) $G_{0}(x)$ is partially complex and is of flag type relative to its algebraic arc components.

If the above conditions hold, then $\mathfrak{X}_{[x]}=\left(\mathcal{P}_{x} \cap \tau \mathcal{P}_{x}\right)+V_{x}=\mathcal{Q}_{[x]}$, so the algebraic arc components of $G_{0}(x)$ coincide with the holomorphic arc components.

PRoof. If $G_{0}(x)$ is measurable, Theorem 9.2 says that it is integrable. If $G_{0}(x)$ is measurable relative to its algebraic arc components, Corollary 9.5 says that it is integrable.

Suppose $G_{0}(x)$ integrable, so (8.6) and Theorem 8.9 show that $\mathscr{T}_{[x]}$ $=\left(\mathcal{P}_{x}+\tau \mathcal{P}_{x}\right)=Q_{[x]}$. As $G_{0}(x)$ is polarized, $\left(\mathcal{P}_{x}+\tau \mathcal{P}_{x}\right)=\left(\mathcal{P}_{x} \cap \tau \mathcal{P}_{x}\right)+\mathcal{V}_{x}$. Thus $\mathscr{T}_{[x]}=\left(\mathcal{P}_{x} \cap \tau \mathcal{P}_{x}\right)+\mathcal{V}_{x}=\mathcal{Q}_{[x]}$, and Theorem 9.2 says that $G_{0}(x)$ both is measurable, and is measurable relative to its algebraic arc components.

If $G_{0}(x)$ is measurable, Theorem 9.2 says it is partially complex and of flag type. If $G_{0}(x)$ is partially complex and of flag type, Theorems 8.9 and 9.7 say that $\left(\odot_{x} \cap \tau \odot_{x}\right)+V_{x} \subset \mathscr{N}_{[x]} \subset\left(\odot_{x} \cap \tau \odot_{x}\right)$, while $\left(\mathcal{P}_{x} \cap \tau \mathcal{P}_{x}\right)+\mathcal{V}_{x}=\left(\mathcal{P}_{x}+\tau \mathcal{P}_{x}\right)$ because $G_{0}(x)$ is polarized; thus $\mathscr{T}_{[x]}$ $=\left(\mathcal{P}_{x} \cap \tau \mathcal{P}_{x}\right)+v_{x}$ and $G_{0}(x)$ is measurable by Theorem 9.2.

If $G_{0}(x)$ is measurable relative to its algebraic arc components, then it is measurable because it is polarized, so it is partially complex by Theorem 9.2; and $v_{x} \subset \mathcal{O}_{[x]}$ by Corollary 9.5 , so $G_{0}(x)$ is of flag type relative to its algebraic arc components by Corollary 9.8 . If $G_{0}(x)$ is partially complex and is of flag type relative to its algebraic arc components, Theorem 8.9 and Corollary 9.8 say that $\left(\odot_{x} \cap \tau \odot_{x}\right)+v_{x}$ $\subset Q_{[x]} \subset \Re_{[x]} \subset\left(\mathcal{P}_{x}+\tau \mathcal{P}_{x}\right)$, while $\left(\mathcal{\odot}_{x} \cap \tau \mathcal{\odot}_{x}\right)+\mathcal{V}_{x}=\left(\odot_{x}+\tau \odot_{x}\right)$ because $G_{0}(x)$ is polarized; thus $Q_{[x]}=\left(\odot_{x} \cap \tau \odot_{x}\right)+v_{x}$ and Corollary 9.5 says that $G_{0}(x)$ is measurable relative to its algebraic arc components.

Q.E.D.

9.10. Corollary. Let $B$ be a Borel subgroup of $G, Y=G / B$ a complex flag manifold, $G_{0}$ a real form of $G$, and $y \in Y$. Then the following conditions are equivalent, and they imply that the algebraic arc components of $G_{0}(y)$ coincide with the holomorphic arc components:

(i) $G_{0}(y)$ is integrable.

(ii) $G_{0}(y)$ is measurable.

(iii) $G_{0}(y)$ is measurable relative to its algebraic arc components.

(iv) $G_{0}(y)$ is partially complex and of flag type. 
(v) $G_{0}(y)$ is partially complex and is of flag type relative to its algebraic arc components.

Proof. Let $\mathfrak{H C}_{\mathrm{C}} \mathbb{Q}_{y}$ be a $\tau$-stable Cartan subalgebra of $\mathrm{g}$. Then $\mathbb{B}_{y}^{r}=\mathscr{H C}=\tau \mathscr{H C}=\tau \mathbb{B}_{y}^{r}$, so $G_{0}(y)$ is polarized and Theorem 9.9 applies. Q.E.D.

9.11. Corollary. Let $X=G / P$ be a complex flag manifold, $G_{0}$ a real form of $G$, and $x \in X$ such that the orbit $G_{0}(x)$ is integrable.

1. $\mathfrak{T}_{[x]}=\left(\mathcal{P}_{x}+\tau \odot_{x}\right)=Q_{[x]}$, so the algebraic arc components of $G_{0}(x)$ coincide with the holomorphic arc components.

2. $G_{0}(x)$ is partially complex and of flag type.

3. $G_{0}(x)$ is measurable if and only if it is polarized.

Proof. Assertion (1) is immediate from (8.6) and Theorem 8.9. Now Theorem 8.9 proves $G_{0}(x)$ partially complex and Theorem 9.7 says that it is of flag type. If $G_{0}(x)$ is measurable, Theorem 9.2 says it is polarized; if $G_{0}(x)$ is polarized, Theorem 9.9 says it is measurable.

Q.E.D.

If $G_{0}(x)$ is an open orbit, then $S_{[x]}=G_{0}(x)$, so it is rather trivial that $G_{0}(x)$ is partially complex and of flag type. Criteria for measurability of $G_{0}(x)$ are given by Theorems 6.3 and 6.7 , the latter being that $P$ and $\tau \rho$ are opposed. We now see that closed orbits are somewhat better behaved.

9.12. Theorem. Let $X=G / P$ be a complex flag manifold, $G_{0}$ a real form of $G$, and $x \in X$ such that $G_{0}(x)$ is the closed $G_{0}$-orbit on $X$. Then $G_{0}(x)$ is measurable, hence partially complex and of flag type.

Proof. Let $B \subset P$ be a Borel subgroup of $G, Y=G / B$ the resulting complex flag manifold, and $\pi: Y \rightarrow X$ the projection. Choose $y \in \pi^{-1}(x)$ such that $G_{0}(y)$ is the closed $G_{0}$-orbit on $Y$.

Let $\mathcal{F}_{0}$ be the Lie algebra of a maximally split Cartan subgroup of $G_{0}, \mathcal{F}_{0}=\mathfrak{J}+\propto$ the decomposition into toroidal and vector parts, and $\mathfrak{F C}=\mathcal{F C}_{0}^{C}$. The $\mathcal{F C}$-root system $\Delta=\Delta_{0} \cup \Delta_{r}$ where $\Delta_{0}$ consists of those roots that vanish on $Q$ and $\Delta_{r}$ consists of those that do not. Ordering the roots, we have

$$
\begin{aligned}
& \text { Iwasawa decomposition } \mathcal{S}_{0}=\Re+a+\Re, \quad \Re^{c}=\sum_{\Delta_{r}^{+}} \varsigma_{\varphi} \\
& \text { minimal parabolic } \mathscr{L}=\Re+a+\Re, \quad \Re^{c}=\Re^{c}+\sum_{\Delta_{0}} \varrho_{\varphi} .
\end{aligned}
$$

Now define a Borel subalgebra $\mathscr{B}^{\prime} \subset \mathcal{G}$ by

$$
B^{\prime}=(J+a+\Re)^{C}+\sum_{\Delta_{r}^{+}} S_{\varphi} .
$$


Then

$$
\tau \mathbb{B}^{\prime}=(\Im+a+\Upsilon \tau)^{c}+\sum_{\Delta_{0}^{-}} \oint_{\varphi}
$$

Thus

(i) $\mathscr{B}^{\prime} \cap \tau \mathbb{B}^{\prime}=(J+Q+\mathscr{T})^{C}$ and

(ii) $\mathbb{B}^{\prime}+\tau \mathbb{B}^{\prime}=\mathscr{L}^{C}$.

Let $y^{\prime} \in Y$ be the point such that $B_{y^{\prime}}=B^{\prime}$. Then (ii) says that $G_{0}\left(y^{\prime}\right)$ is integrable, hence measurable by Corollary 9.10 , hence partially complex and of flag type by Theorem 9.2 or Corollary 9.10. On the other hand, (i) shows that the compact group $K$ with Lie algebra $\mathcal{K}$ is transitive on $G_{0}\left(y^{\prime}\right)$. Thus $G_{0}\left(y^{\prime}\right)$ is the closed $G_{0}$-orbit on $Y$, i.e. $G_{0}\left(y^{\prime}\right)=G_{0}(y)$. That proves our assertions for the case where $P$ is a Borel subgroup of $G$.

Conjugate $\AA^{\prime}$ by an element of $G_{0}$ that sends $y^{\prime}$ to $y$. That done, we may assume that $B_{y}$ is given by the description of $B^{\prime}$ above. Now $P_{x}=B_{y}+D_{0}+D_{r}$ where, for certain subsets $D_{0} \subset \Delta_{0}^{-}$and $D_{r} \subset \Delta_{r}^{-}$,

$$
D_{0}=\sum_{D_{0}} g_{\varphi} \text { and } D_{r}=\sum_{D_{r}} \oint_{\varphi}
$$

Thus

$$
\mathcal{P}_{x}=B_{y}+D_{0}+D_{r} \text { and } \mathbb{B}_{y}^{-u}=\mathcal{P}_{x}^{-u}+D_{0}+D_{r},
$$

and, defining

$$
D_{0}^{-}=\sum_{-D_{0}} S_{\varphi}, \quad D_{r}^{-}=\sum_{-D_{r}} g_{\varphi}, \quad \pi^{-}=\left\{\sum_{\Delta_{\bar{r}}} S_{\varphi}\right\} \cap g_{0},
$$

we have

$$
\tau D_{0}=\mathscr{D}_{0}^{-} \subset \mathscr{B}_{y} \text { and } D_{r}+\tau D_{r} \subset\left(\Re^{-}\right)^{C} .
$$

In particular,

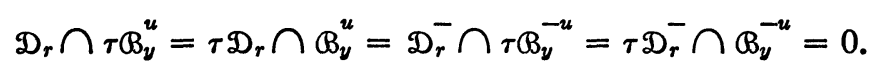

Retaining the notation (9.3), we now have

$$
\begin{aligned}
& v_{y}=\left(\Theta_{y}^{u} \cap \tau{\Theta_{y}^{-u}}^{-u}+\left(\Theta_{y}^{-u} \cap \tau \Theta_{y}^{u}\right)\right.
\end{aligned}
$$

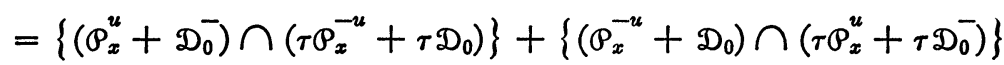

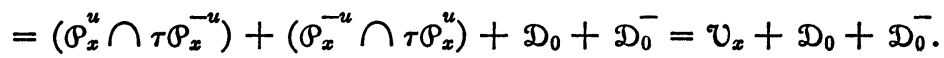

We also compute

$$
\begin{aligned}
\left(\mathscr{P}_{x} \cap \tau \mathcal{P}_{x}\right) & =\left(\mathscr{B}_{y}+\mathscr{D}_{0}+\mathscr{D}_{r}\right) \cap\left(\tau \mathscr{B}_{y}+\tau \mathscr{D}_{0}+\tau \mathscr{D}_{r}\right) \\
& =\left(\mathscr{B}_{y} \cap \tau \mathscr{B}_{y}\right)+\left(\mathscr{D}_{0}+\mathscr{D}_{0}^{-}\right)+\left(\mathscr{D}_{r} \cap \tau \mathscr{D}_{r}\right) .
\end{aligned}
$$




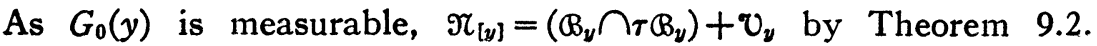
Now define

(9.13a) $\mathscr{W}_{[x]}=\left(\mathcal{P}_{x} \cap \tau \odot_{x}\right)+\vartheta_{x}$.

Our calculations above say that

$$
\begin{aligned}
\mathscr{W}_{[x]} & =\left(\mathscr{B}_{y} \cap \tau \mathscr{B}_{y}\right)+\left(\mathscr{D}_{0}+\mathscr{D}_{0}^{-}\right)+\left(\mathscr{D}_{r} \cap \tau \mathscr{D}_{r}\right)+v_{x} \\
& =\left(\mathscr{B}_{y} \cap \tau \mathscr{B}_{y}\right)+v_{y}+\left(\mathscr{D}_{r} \cap \tau \mathscr{D}_{r}\right) .
\end{aligned}
$$

In other words,

(9.13b) $W_{[x]}=\Re_{[y]}+\left(D_{r} \cap \tau D_{r}\right)$.

As both $\Re_{[y]}$ and $\left(\mathcal{P}_{x} \cap \tau \mathcal{P}_{x}\right)$ are subalgebras of $\mathcal{W}_{[x]}$, and $\left(\mathcal{P}_{x} \cap \tau \mathcal{\odot}_{x}\right)$ $+V_{x}=W_{[x]}=\mathscr{T}_{[y]}+\left(D_{r} \cap \tau D_{r}\right)$ by $(9.13 \mathrm{a}, \mathrm{b})$, the condition for $\mathscr{W}_{[x]}$ to be an algebra is

$$
\left[\mathcal{V}_{x}, \mathscr{D}_{r} \cap \tau \mathscr{D}_{r}\right] \subset W_{[x]}
$$

Observing $D_{r} \subset \mathcal{P}_{x}^{r}$, we calculate

$$
\begin{aligned}
& {\left[v_{x}^{+}, D_{r} \cap \tau D_{r}\right] \subset\left[\rho_{x}^{u} \cap \tau{\rho_{x}^{-u}}^{-u}, \rho_{x}^{r} \cap \tau \mathcal{P}_{x}^{r}\right]}
\end{aligned}
$$

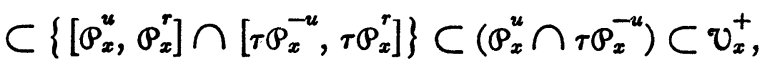

and similarly $\left[\mathcal{V}_{x}^{-}, \mathscr{D}_{r} \cap \tau \mathscr{D}_{r}\right] \subset \mathcal{V}_{x}^{-}$. Thus $\left[\mathcal{V}_{x}, D_{r} \cap \tau D_{r}\right] \subset \mathcal{V}_{x} \subset W_{[x]}$. That proves $\mathscr{W}_{[x]}$ to be an algebra. As it contains the parabolic subalgebra $\Re_{[y]}$ of $\mathcal{G}$, and as $\tau \mathscr{W}_{[x]}=\mathscr{W}_{[x]}$, now

(9.13c) $W_{[x]}$ is a $\tau$-stable parabolic subalgebra of $\mathrm{g}$.

Comparing (9.4) and (9.13a), Theorem 9.2 says that $G_{0}(x)$ is measurable if and only if $W_{[x]}=\mathscr{T}_{[x]}$. As $W_{[x]} \subset \mathscr{T}_{[x]}$ by Lemma 8.4 and Proposition 8.7, Theorem 8.9 says we need only check that $W_{[x]}$ $=\mathfrak{N}_{[x]}$. Define $A$ to be the set of roots such that $\mathfrak{N}_{[x]}=\mathscr{W}_{[x]}+\sum_{A} \mathcal{G}_{\varphi}$ (direct); now the assertion of our theorem is reduced to the statement that $A$ is empty.

Express $\mathfrak{W}_{[x]}^{u}=\sum_{M} \varrho_{\varphi}$ and define $\mu=\sum_{M} \varphi$. Then (9.13c) says that a root space $\oint_{\varphi} \subset W_{[x]}^{r}$ if and only if $\langle\varphi, \mu\rangle=0, \varsigma_{\varphi} \subset W_{[x]}^{u}$ if and only if $\langle\varphi, \mu\rangle>0$, and $\mu=\tau \mu$. In particular, $\langle\varphi, \mu\rangle<0$ for every $\varphi \in A$, and $\langle\varphi, \mu\rangle \geqq 0$ whenever $\mathcal{S}_{\varphi} \subset\left(\mathcal{\odot}_{x} \cap \tau \odot_{x}\right)$.

Let $\varphi \in A$. If $\varphi+\tau \varphi$ is a root, then $\mathcal{S}_{\varphi+\tau} \subset\left(\odot_{x}+\tau \odot_{x}\right)$ implies $\mathcal{G}_{\varphi+\tau \varphi}$ $C\left(\mathcal{P}_{x} \cap \tau \mathcal{P}_{x}\right)$ because $\tau(\varphi+\tau \varphi)=\varphi+\tau \varphi$; then $0 \leqq\langle\varphi+\tau \varphi, \mu\rangle=\langle\varphi, \mu\rangle$ $+\langle\tau \varphi, \mu\rangle=\langle\varphi, \mu\rangle+\langle\tau \varphi, \tau \mu\rangle=2\langle\varphi, \mu\rangle\left\langle 0\right.$, which is absurd; thus $\oint_{\varphi+\tau \varphi}$ $\subset \mathcal{P}_{x}^{-u} \cap \tau \mathcal{P}_{x}^{-u}$, so $\varphi$ is not an $\mathcal{H C}$-root of $\mathfrak{M T}_{[x]}$; that contradicts $\varphi \in A$.

Let $\varphi \in A$. Now $\varphi+\tau \varphi$ is not a root. As $\left(B_{y}+\tau B_{y}\right)=\Re_{[y]} \subset W_{[x]}$ because $G_{0}(y)$ is integrable, we have $G_{\varphi} \subset\left(B_{y}+\tau \AA_{y}\right)$. However $\mathcal{S}_{\varphi} \subset\left(\boldsymbol{P}_{x}+\tau P_{x}\right)$. Replace $\varphi$ by $\tau \varphi$ if necessary so $\oint_{\varphi} \subset{P_{x}}_{x} \mathbb{B}_{y}+D_{0}+D_{r}$. As $D_{0} \subset \tau \otimes_{\nu}$ now $\mathcal{G}_{\varphi} \subset \mathscr{D}_{r}$. As $D_{r} \cap \tau D_{r} \subset W_{[x]}$ now $\mathcal{G}_{\varphi} \subset \tau \mathscr{C}_{x}$. Thus 


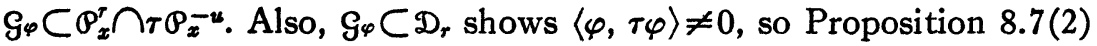
gives us the fact that

(9.14a) $\quad \mathrm{Z}=h_{\varphi} \mathbf{C}+h_{\tau \varphi} \mathbf{C}+\mathcal{G}_{\varphi}+\mathcal{G}_{\tau \varphi}+\mathcal{G}_{-\varphi}+\mathcal{G}_{-\tau \varphi}+\mathcal{G}_{\tau \varphi-\varphi}+\mathcal{G}_{\varphi-\tau \varphi}$

is a $\tau$-stable subalgebra of type $A_{2}$ in $g$ that is contained in $\Re_{[x]}$ and has Cartan subalgebra $\mathfrak{H C}^{\prime}=h_{\varphi} \mathrm{C}+h_{\tau} \boldsymbol{C}$. Define real forms $\mathrm{Z}_{0}=\mathbb{Z} \cap \mathcal{G}_{0}$ and $\mathcal{H}_{0}^{\prime}=\mathcal{H}^{\prime} \cap \mathcal{G}_{0}=\mathfrak{H} \cap \mathcal{Z}_{0}$. Then $\mathcal{F C}_{0}^{\prime}=\mathfrak{J}^{\prime}+Q^{\prime}$ where

(9.14b) $\quad J^{\prime}=\mathfrak{J} \cap \mathfrak{F}^{\prime}=i\left(h_{\varphi}-h_{\tau \varphi}\right) \mathrm{R}$ and $\boldsymbol{Q}^{\prime}=\mathfrak{Q} \cap \mathfrak{F C}^{\prime}=\left(h_{\varphi}+h_{\tau \varphi}\right) \mathrm{R}$.

Note that $\mathrm{J}^{\prime}$ contains a regular element $i\left(h_{\varphi}-h_{\tau \varphi}\right)$ of $\mathrm{Z}$. Now Lemma 4.1 says that $\mathcal{F}_{0}^{\prime}$ is a maximally compact Cartan subalgebra of $Z_{0}$. As $\mathrm{Z}$ is of type $A_{2}$, i.e. $\mathrm{Z}=\$ \&(3, \mathrm{C})$, its real forms are $\$ \&(3, \mathrm{R})$ and the Lie algebras of the groups $\mathrm{SU}(3)$ and $\mathrm{SU}^{1}(3)$. In the latter two cases, a maximally compact Cartan subalgebra of the real form is the Lie algebra of a (compact) torus. Thus $Z_{0} \subset Z$ is $\delta \mathscr{L}(3, R) \subset S \&(3, \mathbf{C})$. That implies that $Z_{0}$ has a split Cartan subalgebra $a^{\prime \prime}$. Now consider the algebra $\mathfrak{H}_{0}+Z_{0}$. It is reductive, and $\mathfrak{F}_{0}$ is its maximally split Cartan subalgebra. Now there exists an element $g$ in the analytic subgroup of $G_{0}$ for $\mathcal{F C}_{0}+Z_{0}$, such that $\operatorname{ad}(g) Q^{\prime \prime} \subset Q$. Replacing $x$ by $g^{-1}(x)$ we obtain $(9.14 \mathrm{a})$ with $\mathfrak{F}_{0}^{\prime}=\mathfrak{Q}^{\prime \prime}$ split, contradicting (9.14b). That contradicts $\varphi \in A$.

We have proved $A$ empty, so $\mathfrak{T}_{[x]}=\mathscr{W}_{[x]}$ algebra, whence $\mathfrak{T}_{[x]}$ $=W_{[x]}$ by Theorem 8.9 , so $G_{0}(x)$ is measurable by $(9.13 \mathrm{a})$ and Theorem 9.2. Q.E.D.

9.15. Corollary. Let $P_{1} \subset P_{2}$ be parabolic subgroups of $G, X_{i}=G / P_{i}$ the resulting complex flag manifolds, and $\pi: X_{1} \rightarrow X_{2}$ the projection $g P_{1} \rightarrow g P_{2}$. Let $G_{0}$ be a real form of $G$. Choose $x_{i} \in X_{i}$ such that $\pi\left(x_{1}\right)=x_{2}$ and $G_{0}\left(x_{i}\right)$ is the closed $G_{0}$-orbit on $X_{i}$. Then holomorphic arc components satisfy $\pi S_{\left[x_{1}\right]}=S_{\left[x_{2}\right]}$.

Proof. Let $B \subset P_{1}$ be a Borel subgroup of $G, Y=G / B$, and $\nu_{i}: Y \rightarrow X_{i}$ the projections. Then $\nu_{2}=\pi \cdot \nu_{1}$. Let $y \in \nu_{1}^{-1}\left(x_{1}\right)$ such that $G_{0}(y)$ is the closed $G_{0}$-orbit on $Y$. Then $\nu_{i}(y)=x_{i}$. Recall $(9.13 \mathrm{~b})$ from the proof of Theorem 9.12 ; it says $W_{\left[x_{i}\right]}=\mathfrak{N}_{[y]}+\left(\right.$ a certain subspace of $\left(P_{i}\right)_{x_{i}}$ $\left.\cap \tau\left(P_{i}\right)_{x_{i}}\right)$. The subsequent portion of the proof of Theorem 9.12 consisted of showing $W_{\left[x_{i}\right]}=\Re_{\left[x_{i}\right]}$. Now $\Re_{\left[x_{i}\right]}=\Re_{[y]}+$ (subspace of $\left(\mathcal{P}_{i}\right)_{x_{i}}$ $\left.\cap \tau\left(\odot_{i}\right)_{x_{i}}\right)$. That proves $\nu_{i} S_{[y]}=S_{\left[x_{i}\right]}$. As $\pi=\nu_{2} \cdot \nu_{1}^{-1}$, it follows that $\pi S_{\left[x_{1}\right]}=S_{\left[x_{2}\right]}$. Q.E.D.

The (rather minor) portion of Theorem 9.12 which says that closed orbits are partially complex, allows us to strengthen Corollary 8.19.

9.16. Corollary. Let $X=G / P$ be a complex flag manifold, $x \in X$, and $G_{0}$ a real form of $G$. Then the following conditions are equivalent. 
(i) $G_{0}(x)$ is the closed $G_{0}$-orbit on $X$.

(ii) The holomorphic arc components of $G_{0}(x)$ are compact.

(iii) The holomorphic arc components of $G_{0}(x)$ are complex flag manifolds.

(iv) $\Re_{[x]}^{u} \subset\left(\odot_{x} \cap \tau \mathcal{P}_{x}\right)$, and $\Re_{[x]}^{r}=\mathfrak{L} \oplus \mathcal{J}$ direct sum of $\tau$-stable ideals, where $g \subset\left(\odot_{x} \cap \tau \odot_{x}\right)$ and $\mathfrak{L} \cap \mathcal{G}_{0}$ is a compactly embedded subalgebra of $\mathcal{G}_{0}$.

Proof. Corollary 8.19 gives equivalence of (i) and (ii). As complex flag manifolds are compact, (iii) implies (ii). Conversely (ii) implies (iii) because $G_{0}(x)$ is of flag type and $S_{[x]}$ is an open $N_{[x], 0^{-}}^{r}$ orbit on the complex flag $N_{[x]}^{r}(x)$.

Given (iv), $\mathcal{J}+\mathscr{T}_{[x]}^{u}$ is an ideal of $\mathscr{\Re}_{[x]}$ contained in the isotropy subalgebra at $x$, so the subgroup $J_{0} \cdot N_{[x], 0}^{u}$ of $G_{0}$ for the subalgebra $\left(\mathcal{I}+\mathfrak{T}_{[x]}^{u}\right) \cap \mathcal{G}_{0}$ must act trivially on $S_{[x]}$. Thus the subgroup $L_{0}$ for $\mathscr{L} \cap \mathcal{G}_{0}$ is transitive on $S_{[x]}$. But $\mathscr{L} \cap \mathcal{G}_{0}$ is compactly embedded, and $G_{0}$ has finite center, so $L_{0}$ is compact. Thus $S_{[x]}$ is compact. Now (iv) implies (ii).

Given (iii), we have $\Re_{[x]}^{u} \subset\left(\odot_{x} \cap \tau \odot_{x}\right)$ and $N_{[x], 0}^{\tau}$ transitive on $S_{[x]}$. Let $J_{0}$ be the kernel of its action, so the Lie algebra $\partial_{0}$ is an ideal of $\mathscr{N}_{[x], 0}^{\tau}$ contained in $\left(\mathcal{P}_{x} \cap \tau \mathcal{P}_{x}\right)_{0}$. Let $\mathscr{L}_{0}$ be a $\tau$-stable complementary ideal, $L_{0}$ the corresponding subgroup of $N_{[x], 0}^{r}$. Then $L_{0}$ is transitive on the flag manifold $S_{[x]}$, acting with finite kernel, so $L_{0}$ is compact. Now (iv) follows with $\mathscr{L}=\mathfrak{L}_{0}^{C}$ and $\mathscr{J}=g_{0}^{C}$. Q.E.D.

We can now complete the results of Corollary 8.20.

9.17. Corollary. Let $X=G / P$ be a complex flag manifold, $G_{0}$ a real form of $G$, and $x \in X$. Then the holomorphic arc components of $G_{0}(x)$ are points if and only if (a) $G_{0}(x)$ is the closed orbit and (b) $\tau \mathcal{P}_{x}^{u} \subset \mathcal{P}_{x}$.

PROOF. If the holomorphic arc components of $G_{0}(x)$ are points, they are compact, so $G_{0}(x)$ is the closed orbit by Corollary 8.19 or Corollary 9.16. Now let $G_{0}(x)$ be the closed orbit, so $\mathcal{I}_{[x]}=\left(\odot_{x} \cap \tau \odot_{x}\right)+\mathcal{V}_{x}$ by Theorem 9.2 ; thus $S_{[x]}$ is a point if and only if $\mho_{x}=0$. As $\vartheta_{x}=\left(\rho_{x}^{u} \cap \tau \odot_{x}^{-u}\right)$ $+\tau\left(\odot_{x}^{u} \cap \tau \odot_{x}^{-u}\right)$, it is 0 if and only if $\tau \odot_{x}^{u} \cap \odot_{x}^{-u}=0$. Thus $S_{[x]}$ is a point if and only if $\tau \odot_{x}^{u} \subset \odot_{x}$. Q.E.D.

It turns out that the proof of Theorem 9.12 does not apply exclusively to the case where $G_{0}(x)$ is closed.

9.18. Proposition. Let $X=G / P$ be a complex flag manifold, $G_{0} a$ real form of $G$, and $x \in X$. Suppose that $\mathcal{G}_{0} \cap \rho_{x}$ contains a maximally split Cartan subalgebra $\mathfrak{H C}_{0}$ of $\mathrm{G}_{0}$. Then the following conditions are equivalent:

(i) $G_{0}(x)$ is measurable.

(ii) $\boldsymbol{N}_{[x]}=\left(\rho_{x} \cap \tau \rho_{x}\right)+v_{x}$.

(iii) $\left(\odot_{x} \cap \tau \odot_{x}\right)+\mathcal{V}_{x}$ is an algebra. 
Proof. Theorem 9.2 is the equivalence of (i) and (ii), and of course (ii) implies (iii). Thus we need only prove that (iii) implies (ii).

Assume (iii) and define $\mathcal{W}_{[x]}=\left(\rho_{x} \cap \tau \odot_{x}\right)+\mathcal{V}_{x}$. The latter agrees with (9.13a). Now $\mathscr{W}_{[x]}$ is an algebra. As $\tau W_{[x]}=W_{[x]}$ and $\left(\odot_{x} \cap \tau \odot_{x}\right)$ $\subset w_{[x]} \subset\left(\odot_{x}+\tau \odot_{x}\right)$, Lemma 8.4 and Proposition 8.7 say $w_{[x]} \subset \mathfrak{N}_{[x]}$. Here we work with the Cartan subalgebra $\mathcal{H}=\mathcal{F C}_{0}^{C}$ of $\mathrm{g}$. Define $A$ to

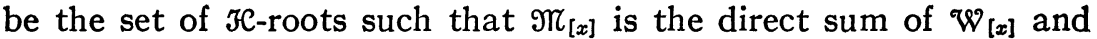
$\sum_{A} \oint_{\varphi}$. As in Theorem 9.12 , we will prove that $A$ is empty.

Let $\varphi$ be a root with $\mathcal{G}_{\varphi} \Phi W_{[x]}$. Then $\varphi$ must be in $\Phi^{r} \cap-\tau \Phi^{u}$, $-\Phi^{u} \cap \tau \Phi^{r}$ or $-\Phi^{u} \cap-\tau \Phi^{u}$ where $\mathcal{P}_{x}=\mathcal{P}_{\Phi}$. Thus $\mathcal{G}_{-\varphi} \subset W_{[x]}$. That proves $W_{[x]}$ parabolic in $\mathcal{G}$. Define $\mu$ to be the sum of all roots $\varphi$ with $\mathcal{G}_{\varphi} \subset W_{[x]}^{u}$. Then $\mu=\tau \mu$ and, for any root $\varphi$,

$$
\begin{array}{ll}
\text { if }\langle\varphi, \mu\rangle>0 & \text { then } \mathcal{S}_{\varphi} \subset W_{[x]}^{u}, \\
\text { if }\langle\varphi, \mu\rangle=0 & \text { then } \mathcal{S}_{\varphi} \subset W_{[x]}^{r}, \\
\text { if }\langle\varphi, \mu\rangle<0 & \text { then } \mathcal{S}_{\varphi} \subset W_{[x]} .
\end{array}
$$

In particular, $\langle\varphi, \mu\rangle\langle 0$ for every $\varphi \in A$, and $\langle\varphi, \mu\rangle \geqq 0$ whenever $\mathrm{S}_{\varphi} \subset\left(\odot_{x} \cap \tau \odot_{x}\right)$.

Let $\varphi \in A$. If $\varphi+\tau \varphi$ is a root, then $\mathcal{G}_{\varphi+\tau} \subset\left(\odot_{x}+\tau \odot_{x}\right)$ would imply $\mathcal{S}_{\varphi+\tau \varphi} \subset\left(\odot_{x} \cap \tau \odot_{x}\right)$, so $0 \leqq\langle\varphi+\tau \varphi, \mu\rangle=2\langle\varphi, \mu\rangle<0$, which is absurd; and $\mathcal{G}_{\varphi+\tau \varphi} \Phi\left(\odot_{x}+\tau P_{x}\right)$ would contradict $\mathcal{S}_{\varphi} \subset \mathfrak{N}_{[x]}$; thus $\varphi+\tau \varphi$ is not a root. As in Proposition 8.7, it follows that there are just two possibilities, given as follows.

Case 1. $\varphi \neq \tau \varphi \neq-\varphi,\langle\varphi, \tau \varphi\rangle=0, \tau \varphi-\varphi$ is not a root, and

$$
\mathbf{Z}=h_{\varphi} \mathbf{C}+h_{\tau \varphi} \mathbf{C}+\mathcal{S}_{\varphi}+\mathcal{S}_{-\varphi}+\mathcal{S}_{\tau \varphi}+\mathcal{S}_{-\tau \varphi}
$$

is a $\tau$-stable semisimple subalgebra of type $A_{1} \oplus A_{1}$ in $\mathscr{W}_{[x]}$.

Case 2. $\varphi \neq \tau \varphi \neq-\varphi,\langle\varphi, \tau \varphi\rangle=\frac{1}{2}\langle\varphi, \varphi\rangle, \tau \varphi-\varphi$ is a root, and

$$
\mathbf{Z}=h_{\varphi} \mathbf{C}+h_{\tau \varphi} \mathbf{C}+\mathcal{S}_{\varphi}+\mathcal{S}_{-\varphi}+\mathcal{S}_{\tau \varphi}+\mathcal{S}_{-\tau \varphi}+\mathcal{S}_{\tau \varphi-\varphi}+\mathcal{S}_{\varphi-\tau \varphi}
$$

is a $\tau$-stable semisimple subalgebra of type $A_{2}$ in $W_{[x]}$.

In both cases, $\mathcal{F C}^{\prime}=h_{\varphi} \mathrm{C}+h_{\tau} \mathrm{C}$ is a Cartan subalgebra of $\mathrm{Z}$, and we

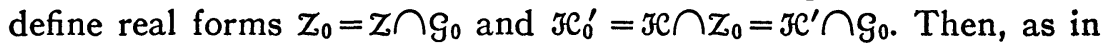
(9.14b), $\mathcal{F C}_{0}^{\prime}=\mathfrak{J}^{\prime}+\mathfrak{Q}^{\prime}$ where

$$
J^{\prime}=i\left(h_{\varphi}-h_{\tau \varphi}\right) \mathbf{R} \text { and } \boldsymbol{Q}^{\prime}=\left(h_{\varphi}+h_{\tau \varphi}\right) \mathbf{R} .
$$

Checking the two cases separately one sees that $i\left(h_{\varphi}-h_{\tau \varphi}\right) \in J^{\prime}$ is a regular element of $\mathcal{Z}$, so Lemma 4.1 assures us that $\mathcal{H}_{0}^{\prime}$ is a maximally compact Cartan subalgebra of $Z_{0}$. We now use the hypothesis that $\mathcal{H C}_{0}$ is maximally split in $\mathcal{G}_{0}$; it allows us to use the argument following (9.14b), and this eliminates Case 2. 
Now we are in Case 1 with $\mathrm{Z}$ given by (9.19a). As $\varphi \in A$ we have $\mathcal{S}_{\varphi} \subset\left(\mathcal{P}_{x}+\tau \odot_{x}\right)$. Replace $\varphi$ by $\tau \varphi$ if necessary for the normalization

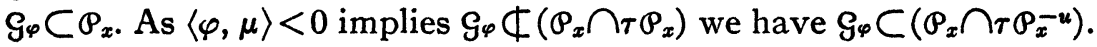
Now $\mathcal{S}_{\varphi} \subset \mathcal{W}_{[x]}$ gives us $\mathcal{G}_{\varphi} \subset\left({\odot_{x}^{r}}_{x} \cap \mathcal{P}_{x}^{-u}\right)$. It follows that

$$
\begin{gathered}
Z \cap \rho_{x}=\mathcal{H}^{\prime}+\mathcal{S}_{\varphi}+\mathcal{S}_{-\varphi}+\mathcal{S}_{-\tau \varphi} \text { and } \\
Z \cap\left(\rho_{x} \cap \tau \mathcal{P}_{x}\right)=\mathcal{H C}^{\prime}+\mathcal{G}_{-\varphi}+\mathcal{S}_{-\tau \varphi} .
\end{gathered}
$$

The first equation of $(9.19 \mathrm{~d})$ says that $Z \cap \rho_{x}$ is parabolic in $Z$, i.e. that the analytic subgroup $Z$ of $G$ for $Z$ has the property that $Z(x)$ is a complex flag manifold. Let $Z_{0}$ denote the analytic subgroup of $G_{0}$ for $Z_{0}$. Now (9.19d) gives us $\operatorname{dim}_{R} Z(x)=2 \operatorname{dim}_{C} Z(x)$ $=2\left\{\operatorname{dim}_{C} Z-\operatorname{dim}_{C}\left(Z \cap P_{x}\right)\right\}=2\{6-5\}=2=6-4=\operatorname{dim}_{C} Z$ $-\operatorname{dim}_{C}\left(Z \cap \mathcal{Q}_{x} \cap \tau \odot_{x}\right)=\operatorname{dim}_{R} Z_{0}-\operatorname{dim}_{R}\left(Z_{0} \cap \rho_{x}\right)=\operatorname{dim}_{R} Z_{0}(x)$. Thus

(9.19e) $\quad Z_{0}(x)$ is open in the complex flag manifold $Z(x)$.

On the other hand, (9.19a) and (9.19c) show that $\mathfrak{K}_{0}^{\prime}$ is a maximally split Cartan subalgebra of $Z_{0}$. The roots positive on $-\left(h_{\varphi}+h_{\tau \varphi}\right) \in a^{\prime}$ are $-\varphi$ and $-\tau \varphi$. Thus we have an Iwasawa decomposition $Z_{0}=\mathcal{K}^{\prime}$ $+a^{\prime}+\mathfrak{x}^{\prime}, \quad \mathfrak{x}^{\prime C}=\mathcal{G}_{-\varphi}+\mathcal{G}_{-r \varphi}$, with $\left(J^{\prime}+a^{\prime}+\mathfrak{r}^{\prime}\right) \subset Z \cap\left(\mathcal{P}_{x} \cap \tau \mathcal{P}_{x}\right)$, so Lemma 3.1 says that $Z_{0}(x)$ is the closed $Z_{0}$-orbit on $Z(x)$. Combining this with $(9.19 \mathrm{e})$, we see that

$$
Z_{0} \text { is the compact real form of } Z \text {, }
$$

so the maximally compact Cartan subalgebras of $Z_{0}$ are compact. That contradicts (9.19c). We conclude that $\varphi \in A$ cannot exist, i.e. that $A$ is empty.

Now $A$ is empty, so $\mathfrak{T}_{[x]}=\mathscr{W}_{[x]}$ algebra; thus $\mathfrak{T}_{[x]}=\mathfrak{T}_{[x]}$ by Theorem 8.9, so $\mathscr{W}_{[x]}=\mathfrak{N}_{[x]}$. Thus (iii) implies (ii), completing the proof of the proposition. Q.E.D.

Let $K$ be a maximal compact subgroup of $G_{0}$. Then every Cartan subalgebra of $\mathcal{S}_{0}$ is maximally split, if and only if rank $\mathcal{G}_{0}=$ rank $\Re$ trank $G_{0} / K$, the latter being the symmetric space rank. The only such cases for which the symmetric space $G_{0} / K$ is irreducible with $G_{0}$ acting almost-effectively are

(9.20a) $G_{0}$ of type $E_{6}$ with $K$ of type $F_{4}$,

(9.20b) $G_{0}$ of type $A_{2 n-1}$ with $K$ of type $C_{n}$,

(9.20c) $G_{0}$ of type $D_{n+1}$ with $K$ of type $B_{n}$, and

(9.20d) $G_{0}$ complex simple but viewed as a real group.

9.21. Corollary. Let $X=G / P$ be a complex flag manifold. Let $G_{0}$ be a real form of $G$ that is locally isomorphic to product of compact simple 
groups and groups given by (9.20) above. Let $x \in X$. Then the following conditions are equivalent:

(i) $G_{0}(x)$ is measurable.

(ii) $\mathfrak{N}_{[x]}=\left(\odot_{x} \cap \tau \odot_{x}\right)+v_{x}$.

(iii) $\left(\odot_{x} \cap \tau \odot_{x}\right)+V_{x}$ is an algebra.

Proof. Let $\mathfrak{H C} \subset \mathcal{P}_{x}$ be a Cartan subalgebra of $\mathcal{G}$ stable under $\tau$. Then the Cartan subalgebra $\mathcal{H}_{0}=\mathfrak{H} \cap \mathcal{G}_{0}$ of $\mathcal{G}_{0}$ is a maximally split Cartan subalgebra of $\mathcal{G}_{0}$, by hypothesis on $G_{0}$. Now Proposition 9.18 applies. Q.E.D.

We end $\$ 9$ with a short study of the case where the complex flag manifold $X=G / P$ is a hermitian symmetric space $G_{u} / P_{u}^{r}$ of compact type relative to the compact real form $G_{u}$ of $G$, and where $G_{0}^{\prime}$ is any real form of $G$. Here we use the notation $G_{0}^{\prime}$ for purposes of reference from $\$ 10$, where $G_{0}$ will denote a real form of $G$ such that $G_{0} / K$ is the noncompact hermitian symmetric space dual to $X=G_{u} / K$.

9.22. Lemma. Let $X=G / P$ be a complex flag manifold. Then the following conditions are equivalent:

(i) $X$ is a hermitian symmetric space $G_{u} / K$.

(ii) $\rho^{u}$ is abelian, i.e. $\left[\rho^{u}, \rho^{u}\right]=0$.

(iii) $\left[\rho^{u}, p^{-u}\right] \subset \rho^{r}$.

Proof. Let $X=G_{u} / K$ hermitian symmetric. Then $\mathcal{K}$ has a central element $z$ that defines the almost complex structure on $X$ by

$$
\begin{gathered}
\operatorname{ad}(z)=\sqrt{-1} \text { on } \rho^{-u}, \quad \operatorname{ad}(z)=-\sqrt{-1} \text { on } \rho^{u}, \\
\operatorname{ad}(z)=0 \text { on } \kappa^{c}=\rho^{r} .
\end{gathered}
$$

If $v, w \in \rho^{u}$ now $\operatorname{ad}(z)[v, w]=[z,[v, w]]=[[z, v], w]+[v,[z, w]]$ $=[\operatorname{ad}(z) v, w]+[v, a d(z) w]=-2 \sqrt{-1}[v, w]$, while $-2 \sqrt{-1}$ is not an eigenvalue of $\operatorname{ad}(z)$; thus $[v, w]=0$; that shows $\rho^{u}$ abelian. If $v \in \rho^{u}$ and $w \in \mathcal{P}^{-u}$ then $\operatorname{ad}(z)[v, w]=[\operatorname{ad}(z) v, w]+[v, \operatorname{ad}(z) w]=0$, so $[v, w] \in \rho^{r}$; that shows $\left[\rho^{u}, \rho^{-u}\right] \subset \mathcal{P r}^{r}$. Now (i) implies (ii) and (iii).

Let $\mathcal{F}=J^{C}$ be a Cartan subalgebra of $\mathcal{G}$ where $\mathcal{J} \subset \mathcal{G}_{u} \cap \odot$. Choose a subset $\Phi$ of a simple $\mathcal{H}$-root system $\Pi$ such that $\mathcal{P}=\mathcal{P}_{\Phi}$. Decompose $\mathcal{G}$ as direct sum of simple ideals $\mathscr{L}_{i}$, so $\mathfrak{H}=\sum \mathfrak{H}_{i}$ where $\mathfrak{H C}_{i}=\mathfrak{H} \cap \mathfrak{L}_{i}$ is a Cartan subalgebra of $\mathscr{L}_{i}$, and $\Pi=U \Pi_{i}$ where $\Pi_{i}$ is a simple $\mathfrak{F C}_{i}$-root system for $\mathfrak{L}_{i}$. Let $\mu_{i}$ be the maximal root of $\mathfrak{L}_{i}$, so $\mu_{i}=\sum_{\mathrm{n}_{i}} n_{\psi} \cdot \psi, n_{\psi}$ integers $\geqq 1$. If $\Pi_{i} \backslash \Phi$ contains a root $\psi_{0}$ with $n_{\psi_{0}}>1$, we have positive roots

$$
\alpha=\sum_{\mathbf{I} i} a_{\psi} \cdot \psi, \quad \beta=\sum_{\mathbf{I} i} b_{\psi} \cdot \psi, \quad \gamma=\alpha+\beta
$$


with $a_{\psi_{0}}>0$ and $b_{\psi_{0}}>0$. If $\Pi_{i} \backslash \Phi$ contains two roots $\psi_{1}$ and $\psi_{2}$, then we have positive roots $\alpha, \beta, \gamma$ as above with $a_{\psi_{1}}>0$ and $b_{\psi_{2}}>0$. In either case $\alpha, \beta, \gamma \in \Phi^{u}$, so

$\mathcal{S}_{\gamma}=\left[\mathrm{S}_{\alpha}, \mathrm{S}_{\beta}\right] \subset\left[\rho^{u}, \rho^{u}\right]$ shows that (ii) fails, and

$\mathcal{G}_{\alpha}=\left[\mathcal{G}_{\gamma}, \mathcal{G}_{-\beta}\right] \subset\left[{\beta^{u}}^{u} \beta^{-u}\right] \cap \rho^{u}$ shows that (iii) fails.

Thus (ii) and (iii) each implies that, for every $i$, either $\Pi_{i} \subset \Phi$ or $\Pi_{i} \backslash \Phi=\left\{\psi_{0}\right\}$ with $n_{\psi_{0}}=1$. It is immediate [10, Lemma 3] that $G_{u} / G_{u} \cap P=G_{u} / P_{u}^{r}$ is a realization of $X$ as a hermitian symmetric space of compact type. Q.E.D.

9.23. Remark. Let $X=G / P$ be a complex flag manifold that is hermitian symmetric. Let $G_{0}^{\prime}$ be any real form of $G, \tau^{\prime}$ conjugation of $\mathcal{G}$ over $\mathcal{G}_{0}^{\prime}$. If $x \in X$ then Lemma 9.22 shows that $\mathcal{W}_{[x]}=\left(\mathcal{P}_{x} \cap \tau^{\prime} \boldsymbol{P}_{x}\right)$ $+\left(\odot_{x}^{u} \cap \tau^{\prime} \odot_{x}^{-u}\right)+\left(\mathcal{P}_{x}^{-u} \cap \tau^{\prime} \odot_{x}^{u}\right)$ is a subalgebra of $\mathcal{G}$. As

$$
\left(\odot_{x} \cap \tau^{\prime} \mathcal{P}_{x}\right) \subset \mathscr{W}_{[x]}=\tau^{\prime} \mathscr{W}_{[x]} \subset\left(\odot_{x}+\tau^{\prime} \mathcal{P}_{x}\right),
$$

Lemma 8.4 says that ${ }^{W_{[x]}} \subset \mathscr{N}_{[x]}$, so Theorem 9.7 says that $G_{0}^{\prime}(x)$ is of flag type. However, Example 8.12 shows that one cannot expect $G_{0}^{\prime}(x)$ to be partially complex without further restriction on $X, x$, or $G_{0}^{\prime}$. In that context we prove

9.24. Proposition. Let $X=G / P$ be a complex flag manifold that is a hermitian symmetric space. Let $G_{0}^{\prime}$ be any real form of $G$. Decompose

$\mathcal{G}=\mathcal{G}^{1} \oplus \cdots \oplus \mathcal{G}^{r}, \quad \rho=\beta^{1} \oplus \cdots \oplus \boldsymbol{p}^{r}, \quad X=X^{1} \times \cdots \times X^{r}$ where the $\mathcal{G}^{i}$ are the minimal $\tau^{\prime}$-stable ideals of $\mathcal{G}, \rho^{i}=P \cap \mathcal{G}^{i}$, and $X^{i}=G^{i} / P^{i}$ quotient of the analytic subgroups of $G$ for $\mathcal{G}^{i}$ and $P^{i}$; so the $\mathcal{G}_{0}^{\prime \prime}=\mathcal{G}_{0}^{\prime} \cap \mathcal{G}^{i}$ are the simple ideals of $\mathcal{G}_{0}^{\prime}$. Then the following conditions are equivalent:

(1) Some open $G_{0}^{\prime}$-orbit on $X$ is a symmetric coset space of $G_{0}^{\prime}$, with invariant nondegenerate kaehler metric that may be of indefinite signature.

$\left(^{\prime}\right)$ Every open $G_{0}^{\prime}$-orbit on $X$ is a symmetric coset space of $G_{0}^{\prime}$, with invariant nondegenerate kaehler metric that may be of indefinite signature.

(2) Some open $G_{0}^{\prime}$-orbit on $X$ is measurable.

(2') Every open $G_{0}^{\prime}$-orbit on $X$ is measurable.

(3) For each index $i, 0 \leqq i \leqq r$, if $\mathcal{G}^{i}$ is simple then either

(3a) the maximal compactly embedded subalgebras $\varkappa^{i} \subset \mathrm{S}_{0}^{\prime i}$ satisfy rank $\mathcal{K}^{i}=$ rank $\mathcal{G}_{0}^{\prime}{ }^{i}$, or

(3b) $X^{i}$ is the grassmannian $\mathrm{U}(2 n) / \mathrm{U}(n) \times \mathrm{U}(n)$ of $n$-planes through the origin in $\mathbf{C}^{2 n}$ with $\mathcal{G}^{i}=S \mathcal{L}(2 n, \mathrm{C})$ and either $\mathcal{S}_{0}^{\prime \prime}=S \mathcal{L}(2 n, \mathrm{R})$ or $\mathcal{G}_{0}^{\prime \prime}=S \mathcal{L}(n, Q)$, or

(3c) $X^{i}$ is the complex quadric $\mathrm{SO}(2 n+2) / \mathrm{SO}(2 n) \times \mathrm{SO}(2)$ with $\mathcal{S}^{i}=S O(2 n+2, \mathrm{C})$ and $\mathcal{S}_{0}^{\prime t}=S \mathcal{O}^{2 t+1}(2 n+2)$ where $0 \leqq t \leqq[n / 2]$. 
(4) For each index $i, g^{i}$ simple, either

(4a) rank $\mathfrak{K}^{i}=$ rank $\mathcal{S}_{0}^{\prime i}$, or

(4b) the noncompact dual of the irreducible compact hermitian symmetric space $X^{i}$ is of tube type, but not isomorphic to an $\mathrm{SO}^{*}(2 l) / \mathrm{U}(l)$ for $l>4$.

Proof. Let $x \in X$ such that $G_{0}^{\prime}(x)$ is open in $X$. If $G_{0}^{\prime}(x)$ is symmetric, say with symmetry $\sigma$ at $x$, then the isotropy subalgebra $\mathcal{G}_{0}^{\prime} \cap \rho_{x}=\left\{v \in \mathcal{G}_{0}^{\prime}: \sigma(v)=v\right\}$ is reductive in $\mathcal{G o}^{\prime}$, so Lemma 6.2 says that $G_{0}^{\prime}(x)$ is measurable. If $G_{0}^{\prime}(x)$ is measurable, Theorem 6.3 says

(i) there is an invariant, possibly indefinite, kaehler metric on $G_{0}^{\prime}(x)$ and

(ii) $\tau^{\prime} \mathcal{P}_{x}^{r}=\mathcal{P}_{x}^{r}$ and $\tau^{\prime} \mathcal{P}_{x}^{u}=\mathcal{P}_{x}^{-u}$.

Choose a compact real form $G_{u}$ of $G$ such that $\mathcal{G}_{0}^{\prime}$ is invariant under the automorphism $\sigma$ of $g$ induced from conjugation of $\mathcal{G}_{u}$ by the symmetry at $x ; \sigma$ is +1 on $\mathcal{G}_{u} \cap \odot_{x}=\left(\odot_{x}^{r}\right)_{u}$ and -1 on a complement; thus $\sigma$ is +1 on $P_{x}^{r}$ and -1 on $P_{x}^{u}+P_{x}^{-u}$; now $\mathcal{S}_{0}^{\prime} \cap \odot_{x}=\left(\right.$ real form of $P_{x}^{r}$ ) $=\left\{v \in \mathcal{S}_{0}^{\prime}: \sigma(v)=v\right\}$, so $G_{0}^{\prime}(x)$ is symmetric. We have proved equivalence of (1) and (2) for $G_{0}^{\prime}(x)$. As (2) and $\left(2^{\prime}\right)$ are equivalent by Theorem 6.7 , we now have equivalence of $(1),\left(1^{\prime}\right),(2)$ and $\left(2^{\prime}\right)$.

In proving (2), (3) and (4) equivalent, it suffices to consider the case where $\mathcal{G}^{\prime}$ is simple. For if $G_{0}(x)$ is open in $X$, and $x=\left(x^{1}, \cdots, x^{r}\right)$ with $x^{i} \in X^{i}$, then Theorem 6.7 shows that $G_{0}^{\prime}(x)$ is measurable if and only if each $G_{0}^{\prime \prime}\left(x^{i}\right)$ is measurable. So we now assume that $g_{0}^{\prime}$ is simple, and we fix $x \in X$ with $G_{0}^{\prime}(x)$ open in $X$.

If $\mathcal{G}$ is not simple, i.e. if $\mathcal{G}_{0}^{\prime}$ is not absolutely simple, then $\mathcal{G}_{0}^{\prime}$ is the real structure of a complex simple Lie algebra $\mathcal{L}, \mathcal{S}=\mathfrak{L} \oplus \mathcal{L}$ with $\mathcal{S}^{\prime}$ embedded diagonally, and $\tau^{\prime}$ acts on $\mathcal{G}$ by $(u, v) \rightarrow(v, u)$. Lemma 9.22

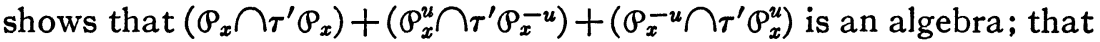
is condition (iii) of Corollary 9.21; thus $G_{0}^{\prime}(x)$ is measurable.

Now let $\rho$ be simple. If the maximal compactly embedded subalgebras $\nVdash \subset \mathcal{G}_{0}^{\prime}$ satisfy rank $\mathscr{K}=$ rank $\mathcal{G}_{0}^{\prime}$, then Corollary 6.4 says that $G_{0}^{\prime}(x)$ is measurable.

Finally suppose $\mathcal{G}$ simple with rank $K<$ rank $\mathcal{G}_{0}^{\prime}$. If $G_{0}^{\prime}(x)$ is measurable, we have the symmetry $\sigma$ of $X=G_{u} / P_{u}^{r}$ at $x$, such that $\sigma \mathcal{G}_{0}^{\prime}=\mathcal{S}_{0}^{\prime}$ and $\mathcal{G}_{0}^{\prime} \cap \mathcal{P}_{x}=\left\{v \in \mathcal{S}_{0}^{\prime}: \sigma(v)=v\right\}$, real form of $\mathcal{P}_{x}^{r}$. Let $\alpha$ be a Cartan involution of $\mathcal{S}_{0}^{\prime}$ that commutes with $\sigma, \mathcal{S}_{0}^{\prime}=\mathfrak{K}+\mathfrak{M}_{0}$ the Cartan decomposition under $\alpha$. Then $\varrho_{u}=\Re+\sqrt{-1} \Re_{0}$, and rank $\Re$ $<$ rank $\mathcal{S}_{u}$, so $\alpha$ carries over to an involutive outer automorphism of $\mathcal{S}_{u}$ that preserves $\left(\odot_{x}^{r}\right)_{u}$, thus acts on $X=G_{u} /\left(P_{x}^{r}\right)_{u}$, and preserves the invariant complex structure on $X$. We apply [11, Theorem 13.3] to $\alpha$ and $X=G_{u} /\left(P_{x}^{r}\right)_{u}$, concluding that either $X=\mathrm{SU}(2 n) / \mathrm{S}(\mathrm{U}(n) \times \mathrm{U}(n))$ with $\alpha$ interchanging the two $\mathrm{U}(n)$ factors, or $X=\mathrm{SO}(2 n+2) / \mathrm{SO}(2 n)$ 
$\times \mathrm{SO}(2)$ with $\alpha$ acting on $\mathrm{SO}(2 n) \times \mathrm{SO}(2) \subset \mathrm{O}(2 n) \times \mathrm{SO}(2)$ as conjugation by some $(Q, P)$ with $\operatorname{det} Q=-1$. The other cases of [11, Theorem 13.3(2)] are eliminated because they are not symmetric. Thus measurability of $G_{0}^{\prime}(x)$ implies alternative (3b) or (3c).

Conversely, suppose that we have alternative (3b) or (3c). Then rank $\Re<$ rank $\mathcal{G}^{\prime}$ shows that $\tau^{\prime}$ induces an outer automorphism of a compact real form $\mathcal{G}_{u}$ of $\mathcal{G}$. Let $\Im$ denote a $\tau^{\prime}$-invariant Cartan subalgebra of $S_{u}$. Choose a $\tau^{\prime}$-invariant Weyl chamber for the Cartan subalgebra $3 \mathcal{C}=J^{C}$ of $\mathcal{G}$. Then $\tau^{\prime}$ acts on the corresponding simple root system II by
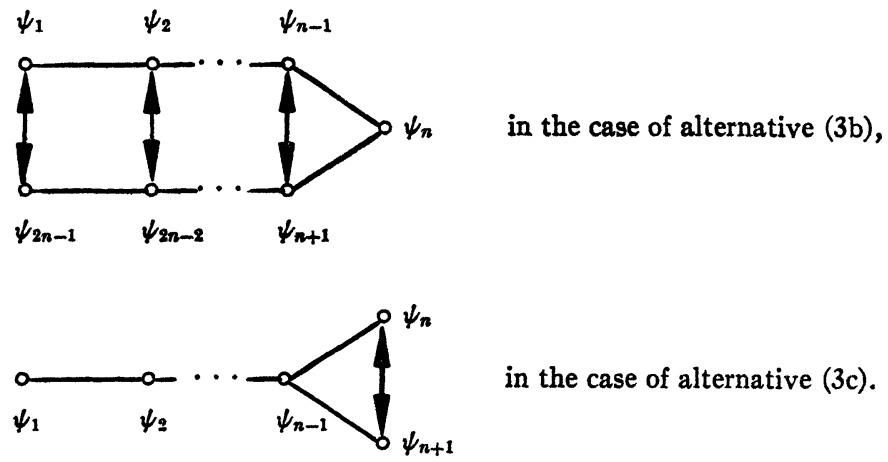

On the other hand, $\odot$ is conjugate to $\rho_{\Phi}$ where, in the notation just above,

$$
\begin{aligned}
& \Phi=\left\{\psi_{1}, \cdots, \psi_{n-1} ; \psi_{n+1}, \cdots, \psi_{2 n-1}\right\} \text { in alternative (3b), } \\
& \Phi=\left\{\psi_{2}, \psi_{3}, \cdots, \psi_{n-1}, \psi_{n}, \psi_{n+1}\right\} \text { in alternative (3c). }
\end{aligned}
$$

Thus $\tau^{\prime} \Phi=\Phi$, so $\tau^{\prime} \mathcal{P}_{\Phi}=\boldsymbol{P}_{\Phi}$. But $\boldsymbol{P}_{\Phi}$ is opposed to itself. Thus $\boldsymbol{P}$ and $\tau^{\prime} \odot$ are opposed. Theorem 6.7 now says that $G_{0}^{\prime}(x)$ is measurable.

We have proved that (2) and (3) are equivalent.

Suppose $\mathcal{G}$ simple with rank $\nVdash<$ rank $\mathcal{G}_{0}^{\prime}$. Then $\mathcal{G}$ has an involutive outer automorphism, so it is of type $A_{l}, D_{l}$ or $E_{6}$. Let $X_{0}$ denote the noncompact dual of $X=G_{u} / P_{u}^{r}$. If $\mathcal{G}$ is of type $A_{l}$, then $X$ is the grassmannian of $k$-planes through the origin in $\mathbf{C}^{l+1}, 2 k \leqq l+1$, and $X_{0}$ is of tube type if and only if $2 k=l+1$. If $g$ is of type $D_{l}$, then either $X=\mathrm{SO}(2 l) / \mathrm{U}(l)$ where $X_{0}$ is of tube type if and only if $l$ is even, or $X=\mathbf{S O}(2 l) / \mathbf{S O}(2 l-2) \times \mathbf{S O}(2)$ complex quadric with $X_{0}$ of tube type. If $\mathcal{G}$ is of type $E_{6}$ then $X_{0}$ is not of tube type. That proves equivalence of (3) and (4), completing the proof of the proposition. Q.E.D.

9.25. Corollary. Let $X=G / P$ be a complex flag manifold that is a 
hermitian symmetric space. Let $G_{0}^{\prime}$ be any real form of $G$ such that the maximal compact subgroups $K \subset G_{0}^{\prime}$ satisfy rank $K=\operatorname{rank} G_{0}^{\prime}$. Then every open $G_{0}^{\prime}$-orbit on $X$ is a symmetric coset space of $G_{0}^{\prime}$ with invariant, nondegenerate, possibly indefinite, kaehler metric.

For that is a special case of alternative (3a) of Proposition 9.24.

\section{Chapter III. Hermitian SYMMETRIC SPACES}

Let $X=G / P$ be a complex flag manifold that is a hermitian symmetric space of compact type. In other words $X=G_{u} / K$, where $G_{u}$ is the compact real form of $G$ and $K=P \cap G_{u}$ (necessarily a compact real form of $P^{r}$ ) is a symmetric subgroup of $G_{u}$. Let $G_{0}$ denote the real form of $G$ such that $X_{0}=G_{0} / K$ is the hermitian symmetric space of noncompact type dual to $X$. $\S 10$ is a complete analysis of the $G_{0}$-orbit structure of $X$ and the holomorphic arc component structure of an arbitrary $G_{0}$-orbit. $\$ 11$ carries that analysis over to a decomposition theory for an arbitrary orbit $G_{0}(x)$ that extends the Siegel domain theory for the convex open orbit $X_{0}$. A summary of the Siegel domain theory for $X_{0}$ and its reformulation as a theory of holomorphic fibrations of $X_{0}$ over its boundary components, is collected in the Appendix to $\$ 11$ for use in our decomposition of $G_{0}(x)$.

In $\$ 10$ we pick out certain points $x_{\Gamma, \Sigma} \in X$ by means of the WolfKorányi partial Cayley transform theory [15], and certain complex subgroups $G_{\Psi \backslash \Gamma} \subset G$ and their $G_{0}$-real forms $G_{\Psi \backslash \Gamma, 0}$, such that the $G_{0}$-orbits on $X$ are just the $G_{0}\left(x_{\Gamma, \Sigma}\right)$ and the holomorphic arc components of $G_{0}\left(x_{\Gamma, \Sigma}\right)$ are just the $k \cdot G_{\Psi \backslash \Gamma, 0}\left(x_{\Gamma, \Sigma}\right)$ where $k \in K$. More precisely let $\Psi$ be a maximal set of strongly orthogonal noncompact positive roots of $\mathcal{G}$ relative to a Cartan subalgebra $J^{C}$ with $J \subset K$. Any two choices of $(\Im, \Psi)$ are $\operatorname{ad}_{G}(K)$-conjugate, $|\Psi|=\operatorname{rank} X_{0}$, and $X_{0}=K \cdot G_{0}[\Psi]\left(x_{0}\right)$ where $x_{0}$ is the base point and $G_{0}[\Psi]\left(x_{0}\right)$ $=\prod_{\Psi} G_{0}[\psi]\left(x_{0}\right)$ polydisc in $X_{0}$. If $\psi \in \Psi$ then $c_{\psi}$ is the Cayley transform on the Riemann sphere $G[\psi]\left(x_{0}\right)$, rotation by $\pi / 2$ carrying the south pole $x_{0}$ up to the equator. If $B \subset \Psi$ then $c_{B}=\prod_{B} c_{\psi}$. If $\Gamma, \Sigma \subset \Psi$ then $x_{\Gamma, \Sigma}=c_{\Gamma} c_{\Sigma}^{2} x_{0}$ and $G_{\Psi \backslash \Gamma}$ is the derived group of the centralizer of $\Im^{C}+\sum_{\psi \perp \Gamma} g_{\psi}$ in $G$.

Theorem 10.6 says that the $G_{0}$-orbits on $X$ are the $G_{0}\left(x_{\Gamma, \Sigma}\right), \Gamma$ and $\Sigma$ disjoint in $\Psi$, and gives criteria for $G_{0}\left(x_{\Gamma^{\prime}, \Sigma^{\prime}}\right)=G_{0}\left(x_{\Gamma, \Sigma}\right)$ and $G_{0}\left(x_{\Gamma^{\prime}, \Sigma^{\prime}}\right) \subset \overline{G_{0}\left(x_{\Gamma, \Sigma}\right)}$. If $\left\{r_{1}, \cdots, r_{p}\right\}$ are the ranks of the irreducible factors of $X_{0}$, it follows that there are precisely $\frac{1}{2} \sum_{1 \leq i \leq p}\left(r_{i}+1\right)\left(r_{i}+2\right)$ $G_{0}$-orbits on $X$. The open $G_{0}$-orbits are the $G_{0}\left(x_{\phi, \Sigma}\right)$; they are $\sum_{1 \leq j \leq p}\left(r_{j}+1\right)=|\Psi|+p$ in number, are indefinite-hermitian symmetric spaces of $G_{0}$, and are indefinite metric versions of $X_{0}$. Theorem 
10.9 says that $G_{\Psi \backslash \Gamma}\left(x_{\Gamma, \Sigma}\right)=G_{\Psi \backslash \Gamma}\left(x_{\Gamma, \phi}\right)$ is a compact totally geodesic hermitian symmetric subspace of rank $|\Psi \backslash \Gamma|$ in $X$, that the $G_{\Psi \backslash \Gamma, 0}\left(x_{\Gamma, \Sigma}\right), \Gamma \cap \Sigma=\phi$, are the open $G_{\Psi \backslash \Gamma, 0}$-orbits on it, that the holomorphic arc components of $G_{0}\left(x_{\Gamma, \Sigma}\right)$ are the $k \cdot G_{\Psi \backslash \Gamma, 0}\left(x_{\Gamma, \Sigma}\right)$ with $k \in K$, and that the complex Lie algebra normalizer $\mathfrak{N}_{\left[x_{\Gamma, \Sigma}\right]}=\mathfrak{N}_{\Gamma, \Sigma}$ of $G_{\Psi \backslash \Gamma, 0}\left(x_{\Gamma, \Sigma}\right)$ depends only on $\Gamma$. The method is rather delicate; Theorem 8.9 is used to show $\mathfrak{T}_{\Gamma, \Sigma}$ independent of $\Sigma$, and results from Wolf-Korányi [15] are used to determine $\mathfrak{T}_{\Gamma, \phi}$. In particular every holomorphic arc component of every $G_{0}$-orbit is indefinite-hermitian symmetric, so every $G_{0}$-orbit is measurable.

In $\$ 11$ we work out a decomposition:

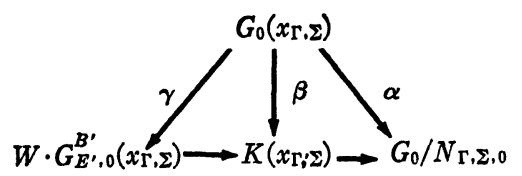

There $\alpha$ is the $K$-equivariant fibration whose fibres are the holomorphic arc components of $G_{0}\left(x_{\Gamma, \Sigma}\right) . \beta$ is a $K$-equivariant fibration whose restriction to a holomorphic arc component is a holomorphic fibration over the maximal compact subvariety of that component; the fibres of $\beta$ are hermitian symmetric spaces $k c_{\Gamma} c_{\Sigma}^{2}\left\{G_{\Psi \backslash(\Gamma \cup \Sigma), 0}\left(x_{0}\right)\right.$ $\left.X G_{\Sigma, 0}^{\Sigma}\left(x_{0}\right)\right\}$ of noncompact type whose second factor is the tube part of $G_{\Sigma, 0}\left(x_{0}\right)$. And $\gamma$ is an a.e. analytic measurable bundle whose restriction to $c_{\Gamma} c_{\Sigma}^{2}\left\{G_{\Psi \backslash(\Gamma \cup \Sigma), 0}\left(x_{0}\right) \times G_{\Sigma, 0}^{\Sigma}\left(x_{0}\right)\right\}$ is a holomorphic fibration over a bounded symmetric domain, any of the Siegel domain realizations of type III for a boundary component of $G_{\Psi \backslash(\Gamma \cup \Sigma), 0}\left(x_{0}\right) \times G_{\Sigma, 0}^{\Sigma}\left(x_{0}\right)$. The Siegel domain theory used in $\gamma$ is in the Appendix to $\S 11$. The fibration $\beta$ plays two roles. It gets rid of compact subvarieties of holomorphic arc components so that some function theory is possible despite the injunction of Theorem 5.7, and it allows construction of the "generalized Siegel domain" $\gamma$ in a manner whose discontinuities do not interfere with integration.

Notes For CHAPter III. The results of Chapter III depend in an essential manner, both on the results of Chapter II, and on the earlier work of Wolf-Korányi [15]. The latter contains the theorems of $\$ 10$ for those $G_{0}$-orbits that are in the topological closure of $X_{0}$.

Enumeration of the $G_{0}$-orbits on $X$ (Theorem 10.6) was first worked out by Takeuchi [9]. Using the partial Cayley transforms of WolfKorányi [15] it is not difficult to see that every $G_{0}$-orbit must be one of the $G_{0}\left(x_{r, \Sigma}\right)$, so the problem is reduced to that of finding a criterion for $G_{0}\left(x_{\Gamma^{\prime}, \Sigma^{\prime}}\right)=G_{0}\left(x_{\Gamma, \Sigma}\right)$. There, a little serious thought reduces the question to the case $\Gamma^{\prime}=\phi=\Gamma$ of open orbits. Takeuchi [9] settles the 
latter by picking out the maximal compact subvarieties of open orbits and counting them with the aid of some Morse theory results on cell decompositions from his thesis [8]. We find it more convenient to apply Theorem 4.9 and count some double cosets.

The fibration $\beta: G_{0}\left(x_{\Gamma, \Sigma}\right) \rightarrow K\left(x_{\Gamma, \Sigma}\right)$ was worked out by Takeuchi [9] for the case $\Gamma=\phi$ of open orbits, where holomorphic arc components are not needed; part 3 of Theorem 11.8 answers (in general) the triviality question on $\beta$ that Takeuchi raised in his special case. A key to our result Theorem 11.8 on $\beta$ is Lemma 11.6, which was proved by Takeuchi [9], for the case $\Gamma=\phi$ of open orbits, in a different way.

Except for the overlap with Wolf-Koranyi [15] and Takeuchi [9] described above, the material of $\$ 10$ and $\S 11$ was worked out by this author in the fall and winter of 1968.

10. Hermitian symmetric spaces: orbit structure and holomorphic arc components. Let $X=G_{u} / \mathcal{K}$ be a hermitian symmetric space of compact type. Then $X$ is a flag manifold $G / P$ where $G_{u}$ is the compact real form of $G$ and $K=P \cap G_{u}$ is the compact real form of $P^{r}$. Now let $X_{0}=G_{0} / K$ be the dual hermitian symmetric space of noncompact type; if $\Theta_{u}=\mathscr{K}+\mathfrak{N}_{u}$ Cartan decomposition, then $\mathcal{G}_{0}=\mathfrak{K}+\mathfrak{N}_{0}$, $\mathfrak{T}_{0}=\sqrt{-1} \mathfrak{M}_{u}$, real form of $\varrho$ such that $K=P \cap G_{0}$, maximal compact subgroup of $G_{0}$. The "Borel embedding" of $X_{0}$ in $X$ is the embedding $b: X_{0} \rightarrow X$ of $X_{0}$ as an open $G_{0}$-orbit on $X$, given by $b(g K)=g P$ for $g \in G_{0}$. Let $x_{0}$ denote the identity coset in $X=G / P$, so $b\left(X_{0}\right)=G_{0}\left(x_{0}\right)$. We map $\mathcal{P}^{-u}$ on to a dense open subset of $X$ by $\xi(z)=\exp (z) \cdot x_{0}$. Then the "Harish-Chandra embedding" of $X_{0}$ as a bounded domain $D$ in a complex euclidean space $\rho^{-u}$ is given by $D=\kappa\left(X_{0}\right)$ where $\kappa(g K)$ $=\xi^{-1} b(g K)$, and $\xi: D \cong G_{0}\left(x_{0}\right)$ relates the two embeddings. One views the inclusions $\left\{\xi(D)=G_{0}\left(x_{0}\right)\right\} \subset \xi\left(\beta^{-u}\right) \subset X$ as a generalization of the classical (unit disc) $\subset$ (complex line) $\subset$ (Riemann sphere).

Bott-Korányi (see [2, Theorem 3.6]) used convexity properties of $D$ in $\beta^{-u}$ to identify the Bergman-Šlov boundary of $G_{0}\left(x_{0}\right)$ in $X$ as the unique closed $G_{0}$-orbit in the topological boundary $\partial G_{0}\left(x_{0}\right)$ of $G_{0}\left(x_{0}\right)$ in $X$. Korányi-Wolf [2] studied the structure of the BergmanŠilov boundary and its role in the realization of $X_{0}$ as a Siegel Domain of type II. Then Wolf-Korányi [15] made full use of the convexity of $D$ in $\rho^{-u}$ to obtain the $G_{0}$-orbit structure of $\partial G_{0}\left(x_{0}\right)$, the holomorphic arc components of $\partial G_{0}\left(x_{0}\right)$ (there called the "boundary components" of $G_{0}\left(x_{0}\right)$ in $X$ ), and the realizations of $X_{0}$ as Siegel Domains of type III. There, it was shown that every holomorphic arc component of $\partial G_{0}\left(x_{0}\right)$ lay in a boundary orbit; so the holomorphic arc components of $\partial G_{0}(x)$ are the same as the holomorphic arc components of orbits $G_{0}(x), x \in \partial G_{0}\left(x_{0}\right)$. Having seen Korányi-Wolf [2] but not Wolf- 
Korányi [15], Maebashi [6] enumerated the $G_{0}$-orbits on $X$ for the case where $X_{0}$ is the Siegel upper half plane $\operatorname{Sp}(n, \mathbf{R}) / \mathrm{U}(n)$. Finally, Takeuchi [3] extended part of Wolf-Korányi [15], enumerating the $G_{0}$-orbits on $X$ in general by means of a recursive procedure based on a counting of the open $G_{0}$-orbits; the latter consisted of describing a set consisting of just one maximal compact subvariety from each open $G_{0}$-orbit as the critical set of a certain function on $X$. Nonconvexity of the general open $G_{0}$-orbit on $X$ prevented Takeuchi from extending the boundary component theory. That difficulty is avoided by our notion of holomorphic arc component developed in $\$ \S 8$ and 9 , and this allows us to give a complete treatment of the $G_{0}$-orbit structure of $X$.

We establish notation for hermitian symmetric spaces.

$X_{0}$ : hermitian symmetric space of noncompact type.

$G_{0}$ : largest connected group of isometries of $X_{0}$.

$x_{0}$ : fixed "base point" in $X_{0}$.

$K$ : isotropy subgroup of $G_{0}$ at $x_{0}$, maximal compact in $G_{0}$.

$\sigma$ : Cartan involution ad(s), where $s$ is the symmetry of $X_{0}$ at $x_{0}$.

Let $\nVdash C g_{0}$ denote the Lie algebras of $K \subset G_{0}$. Then

$\mathcal{S}_{0}=\Re+\mathfrak{N}_{0}$ : Cartan decomposition into $( \pm 1)$-eigenspaces of $\sigma$.

$\mathcal{S}=\mathcal{S}_{0}^{C}=\Re^{C}+\mathscr{N}$ complexification where $\mathfrak{N}=\mathfrak{N}_{0}^{C}$.

$\mathcal{S}_{u}=\mathfrak{K}+\mathfrak{N}_{u}, \mathfrak{M}_{u}=i \mathfrak{M}_{0}$, compact real form of $\mathcal{G}$.

$G$ : adjoint group of $\mathcal{G}$, so $G_{0}$ is the analytic subgroup for $\mathcal{G}_{0}$.

$G_{u}$ : analytic subgroup of $G$ for $\mathcal{S}_{u}$, compact real form of $G$.

$X: G_{u} / K$, hermitian symmetric space, compact dual of $X_{0}$.

J: Cartan subalgebra of $g_{0}\left(\right.$ or $g_{u}$ ) contained in $\varkappa$.

$\Delta: \mathfrak{J}^{C}$-root system of $\mathcal{G}$.

$\Delta_{K}$ : compact roots, i.e. $\Im^{C}$-root system of $\aleph^{C}$.

$\Delta_{M}$ : noncompact roots, i.e. roots $\varphi$ such that $g_{\varphi} \subset \mathfrak{T}$.

$z$ : central element of $\pi$ such that $J=\left.\operatorname{ad}(z)\right|_{\mathfrak{R}}$ is the almost complex structure of $X_{0}$ and $X$.

$\mathfrak{N}^{ \pm}:(\mp \sqrt{-1})$-eigenspaces of $J=\left.\operatorname{ad}(z)\right|_{\mathfrak{M}}$.

$\odot: \mathfrak{K}^{C}+\mathfrak{T}^{+}$, parabolic subalgebra of $\mathcal{G}$ defined by $i z$.

$P$ : parabolic subgroup of $G$ with Lie algebra $P$.

We observe $\beta^{r}=\Re^{c}, \beta^{u}=\mathscr{N}^{+}$and $\beta^{-u}=\mathfrak{N}^{-}$. Theorem 5.4 says $G_{0} \cap P=K=G_{u} \cap P$. Thus $G_{u}$ is transitive on the complex flag manifold $G / P$ with isotropy subgroup $K$. That identifies $X=G_{u} / K$ with the complex flag manifold $G / P$, providing the Borel embedding $b: X_{0} \rightarrow X$ by $b(g K)=g P$ for $g \in G_{0}$. Now we identify $X_{0}$ with $b\left(X_{0}\right)$, thus identifying the base point $x_{0}$ with the identity coset of $X$, thus identifying $X_{0}$ with the open orbit $G_{0}\left(x_{0}\right)$. 
Choose a simple root system $\Pi$ such that $\rho=\mathcal{P}_{\Phi}$ for some subset $\Phi \subset \Pi$. Then $\Phi$ consists of the compact simple roots and $\Pi \backslash \Phi$ the noncompact simple roots. There is one noncompact simple root for each simple ideal of $\mathrm{G}$. Note

$$
\Phi^{r}=\Delta_{K}, \quad \Phi^{u}=\Delta_{M}^{+}, \quad-\Phi^{u}=\Delta_{M}^{-} .
$$

Two roots $\varphi$ and $\psi$ are strongly orthogonal if neither of $\varphi \pm \psi$ is a root; in that case $\langle\varphi, \psi\rangle=0$. Now we define

$\Psi$ : maximal set of strongly orthogonal positive noncompact roots by $\Psi=\left\{\Psi_{1}, \cdots, \Psi_{r}\right\}$ where $\Psi_{j+1}$ is the lowest element of $\Delta_{M}^{+}$strongly orthogonal to each of $\left\{\psi_{1}, \cdots, \psi_{j}\right\}$. Thus, for each simple ideal of $\mathcal{G}$, the noncompact simple root is the first element of $\Psi$ that is a root of the ideal. The number $r=|\Psi|$ is the common symmetric space rank of $X_{0}$ and $X$. Define a real basis of $\mathfrak{T l}_{0}$ consisting of all

$$
x_{\varphi, 0}=e_{\varphi}+e_{-\varphi} \text { and } y_{\varphi, 0}=-i\left(e_{\varphi}-e_{-\varphi}\right), \varphi \in \Delta_{M}^{+},
$$

where $e_{ \pm \varphi} \in \mathcal{S}_{ \pm \varphi}$ are normalized by $\left[e_{\varphi}, e_{-\varphi}\right]=h_{\varphi} \in i$. Then $\mathfrak{T}_{u}$ has a real basis consisting of all

$$
x_{\varphi}=i\left(e_{\varphi}+e_{-\varphi}\right) \quad \text { and } \quad y_{\varphi}=e_{\varphi}-e_{-\varphi}, \quad \varphi \in \Delta_{M}^{+} .
$$

That gives us

$$
\begin{aligned}
& a_{0}: \sum_{\Psi} R x_{\psi, 0}, \quad \text { maximal abelian subspace of } \mathfrak{M}_{0} . \\
& a_{u}: i a_{0}=\sum_{\Psi} R x_{\psi}, \quad \text { maximal abelian subspace of } \mathfrak{M}_{u} .
\end{aligned}
$$

For every noncompact positive root $\varphi$ we compute

$$
\begin{array}{cl}
J x_{\varphi, 0} & =\left[z, x_{\varphi, 0}\right]=y_{\varphi, 0}, \quad J y_{\varphi, 0}=\left[z, y_{\varphi, 0}\right]=-x_{\varphi, 0}, \quad\left[x_{\varphi, 0}, y_{\varphi, 0}\right]=2 i h_{\varphi}, \\
J x_{\varphi} & =\left[z, x_{\varphi}\right]=y_{\varphi}, \quad J y_{\varphi}=\left[z, y_{\varphi}\right]=-x_{\varphi}, \quad\left[x_{\varphi}, y_{\varphi}\right]=-2 i h_{\varphi} .
\end{array}
$$

In particular, using strong orthogonality of the roots in $\Psi$,

$$
\left[\mathfrak{Q}_{0}, J \mathfrak{Q}_{0}\right]=\left[\mathfrak{Q}_{u}, J \mathfrak{Q}_{u}\right]=\sum_{\Psi}\left(i h_{\psi}\right) \mathbf{R} .
$$

That gives

(10.1a) $J=J^{+}+J^{-}$orthogonal direct sum, where

(10.b) $J^{-}=\left[a_{0}, J a_{0}\right]$ and $J^{+}$is the centralizer of $a_{0}$ in $J$.

For every subset $\Gamma \subset \Psi$ we define

(10.2a) $\Im_{\Gamma^{-}}^{-}=\sum_{\bar{\Psi} \backslash \mathrm{r}}\left(i h_{\psi}\right) \mathbf{R}$, so $\Im_{\bar{\phi}}^{-}=J^{-}$and $\Im_{\bar{\Psi}}^{-}=0$;

(10.2b) $a_{\Gamma}=\sum_{\mathrm{r}}\left(x_{\gamma, 0}\right) \mathrm{R}$, so $a_{\phi}=0$ and $a_{\Psi}=a_{0}$;

(10.2c) $\mathfrak{H C}_{\Gamma}=\mathfrak{J}^{+}+\mathfrak{J}_{\Gamma}^{-}+\mathfrak{Q}_{\Gamma}$, so $\mathfrak{H}_{\phi}=\mathfrak{J}$ and $\mathfrak{K C}_{\Psi}=\mathfrak{J}^{+}+Q_{0}$. 
Note that (10.2c) is an orthogonal direct sum. The $\mathfrak{H}_{\Gamma}$ are the Cartan subalgebras of $g_{0}$ with which we shall work. They are related by the partial Cayley transforms [15] defined by

(10.3a) $c_{\psi}=\exp \left((\pi / 4) y_{\psi}\right) \in G_{u}$ for every $\psi \in \Psi$, and

(10.3b) $c_{\Gamma}=\prod_{r} c_{\gamma} \in G_{u}$ for every $\Gamma \subset \Psi$.

Now we have

(10.4a) $\operatorname{ad}\left(c_{\psi}\right): y_{\psi} \rightarrow y_{\psi}, x_{\psi} \rightarrow 2 i h_{\psi}, 2 i h_{\psi} \rightarrow-x_{\psi}$; so

(10.4b) $\operatorname{ad}\left(c_{\Gamma^{\prime}} \backslash \Gamma\right) \mathcal{F C}_{\Gamma}^{C}=\mathcal{F}_{\Gamma^{\prime}}^{C}$ for $\Gamma \subset \Gamma^{\prime} \subset \Psi$, and

(10.4c) $\operatorname{ad}\left(c_{\Sigma}\right)^{2} \mathcal{F}_{\Gamma}=\mathfrak{H}_{\Gamma}$ for $\Gamma, \Sigma \subset \Psi$.

We use the partial Cayley transforms to define points

(10.5) $x_{\Gamma, \Sigma}=c_{\Gamma} c_{\Sigma}^{2} x_{0} \in X$ whenever $\Gamma, \Sigma \subset \Psi$.

Now we can state the decomposition of $X$ into $G_{0}$-orbits.

10.6 Theorem. Decompose $\mathcal{G}=\mathcal{G}^{1} \oplus \cdots \oplus \mathcal{G}^{p}$ direct sum of simple ideals, so $\Delta=\Delta^{1} \cup \ldots \cup \Delta^{p}$ where $\Delta^{k}$ is the root system of $\mathrm{G}^{k}$.

1. The $G_{0}$-orbits on $X$ are just the $G_{0}\left(x_{\Gamma, \Sigma}\right), \Gamma$ and $\Sigma$ disjoint.

2. Orbits $G_{0}\left(x_{\Gamma, \Sigma}\right)$ and $G_{0}\left(x_{\Gamma^{\prime}, \Sigma^{\prime}}\right)$ are equal if and only if, for $1 \leqq k \leqq p$, $\left|\left(\Sigma^{\prime} \backslash \Gamma^{\prime}\right) \cap \Delta^{k}\right|=\left|(\Sigma \backslash \Gamma) \cap \Delta^{k}\right|$ and $\left|\Gamma^{\prime} \cap \Delta^{k}\right|=\left|\Gamma \cap \Delta^{k}\right|$. In particular, the number of $G_{0}$-orbits on $X$ is $\frac{1}{2} \sum_{1 \leq k \leq p}\left(r_{k}+1\right)\left(r_{k}+2\right)$ where $r_{k}=\left|\Psi \cap \Delta^{k}\right|$.

3. $G_{0}\left(x_{\Gamma^{\prime}, \Sigma^{\prime}}\right)$ is in the closure of $G_{0}\left(x_{\Gamma, \Sigma}\right)$ if and only if, for $1 \leqq k \leqq p$, $\left|\left(\Sigma^{\prime} \backslash \Gamma^{\prime}\right) \cap \Delta^{k}\right| \leqq\left|(\Sigma \backslash \Gamma) \cap \Delta^{k}\right|$ and $\left|(\Sigma \cup \Gamma) \cap \Delta^{k}\right| \leqq\left|\left(\Sigma^{\prime} \cup \Gamma^{\prime}\right) \cap \Delta^{k}\right|$.

4. $G_{0}\left(x_{\Gamma, \Sigma}\right)$ is open in $X$ if and only if $\Gamma=\phi$ empty set. In particular there are precisely $\sum_{1 \leq k \leq p}\left(r_{k}+1\right)=|\Psi|+p$ open $G_{0}$-orbits on $X$.

5. $G_{0}\left(x_{\Psi, \phi}\right)$ is the closed $G_{0}$-orbit on $X$, Bergman-Silov boundary of $X_{0}$ in $X$, contained in the closure of every $G_{0}$-orbit.

The corresponding result for Cartan subalgebras is

10.7. Theorem. Let $\mathcal{G}^{k}$ and $\Delta^{k}$ be as in Theorem 10.6.

1. $\mathcal{H}_{\Gamma}$ is maximally compact among the Cartan subalgebras of $\mathrm{G}_{0}$ contained in $\beta_{x_{\Gamma, \Sigma}}, \Gamma \cap \Sigma=\phi$, and every Cartan subalgebra of $\mathcal{S}_{0}$ is conjugate to some $\mathfrak{F C}_{\mathrm{r}}$.

2. $\mathfrak{H}_{\Gamma}$ and $\mathfrak{K}_{\Gamma}$ are conjugate in $\mathcal{S}_{0}$, if and only if, for $1 \leqq k \leqq p$, $\left|\Gamma \cap \Delta^{k}\right|=\left|\Gamma^{\prime} \cap \Delta^{k}\right|$.

3. $\mathcal{F C}_{\Gamma}$ centralizes a $\mathcal{G}_{0}$-conjugate of $\mathcal{J}_{\Gamma^{\prime}}^{-}$, if and only if, for $1 \leqq k \leqq p$, $\left|\Gamma \cap \Delta^{k}\right| \leqq\left|\Gamma^{\prime} \cap \Delta^{k}\right|$.

4. $\mathcal{H C}_{\Gamma}$ is maximally compact in $\mathrm{G}_{0}$ if and only if $\Gamma=\phi$.

5. $\mathfrak{H}_{\Gamma}$ is maximally split in $\mathcal{G}_{0}$ if and only if $\Gamma=\Psi$.

We will prove Theorems 10.6 and 10.7 as we decompose a $G_{0}$-orbit into holomorphic arc components. To express that decomposition, note that

$$
\Im^{c}+\sum_{\varphi \perp \Gamma} \oint_{\varphi} \text { is the centralizer of } \overline{J_{\Psi} \backslash \Gamma} \text { in } \mathcal{G}
$$


Now define

(10.8a) $\mathcal{G}_{\Psi \backslash \Gamma}$ is the derived algebra of $\mathfrak{J}^{C}+\sum_{\varphi \perp \Gamma} \mathcal{G}_{\varphi}$,

(10.8b) $\mathcal{G}_{\Psi \backslash \Gamma, 0}$ is the real form $\mathcal{G}_{0} \cap \mathcal{G}_{\Psi \backslash \Gamma}$ of $\mathcal{S}_{\Psi \backslash \Gamma}$, and

(10.8c) $G_{\Psi \backslash \Gamma}$ and $G_{\Psi \backslash \Gamma, 0}$ are the analytic subgroups for $\mathcal{G}_{\Psi \backslash \Gamma}$ and $\mathcal{S} \Psi \backslash \Gamma, 0$.

10.9. THEOREM. Let $\Gamma$ and $\Sigma$ be arbitrary subsets of $\Psi$.

1. $G_{\Psi \backslash \Gamma}\left(x_{\Gamma, \Sigma}\right)=c_{\Gamma} c_{\Sigma}^{2} G_{\Psi \backslash \Gamma}\left(x_{0}\right)$, compact totally geodesic hermitian symmetric subspace of $X$; its symmetric space rank is $|\Psi \backslash \Gamma|$; relative to the Cartan subalgebra $\mathfrak{J} \cap \mathcal{G}_{\Psi \backslash \Gamma, 0}, \Psi \backslash \Gamma$ is a maximal set of strongly orthogonal noncompact roots of $\mathcal{G}_{\Psi \backslash \Gamma, 0}$.

2. $G_{\Psi \backslash \Gamma, 0}\left(x_{\Gamma, \Sigma}\right)$ is an open $G_{\Psi \backslash \Gamma, 0-o r b i t}$ on the symmetric complex flag manifold $G_{\Psi \backslash \Gamma}\left(x_{\Gamma, \Sigma}\right)$. It is an indefinite-hermitian symmetric space of $G_{\Psi \backslash \Gamma, 0}$; if $\Gamma \cap \Sigma=\phi$, then its invariant kaehler metric is positive definite if and only if $\Sigma$ is a (possibly empty) union of sets $(\Psi \backslash \Gamma) \cap \Delta^{k}$ for which $G_{0}^{k}\left(x_{0}\right)$ is of tube type. Its isotropy subalgebra $\mathcal{S}_{\Psi \backslash \Gamma, 0} \cap \odot_{x_{\Gamma, \Sigma}}$ at $x_{\Gamma, \Sigma}$ has complexification $\operatorname{ad}\left(c_{\Sigma}^{2}\right)\left\{\Theta_{\Psi \backslash \Gamma} \cap \rho_{x_{\Gamma}, \phi}\right\}$.

3. The holomorphic arc components of $G_{0}\left(x_{\Gamma, \Sigma}\right)$ are just the $g \cdot G_{\Psi \backslash \Gamma, 0}\left(x_{\Gamma, \Sigma}\right), g \in K . I f$

(10.10a) $N_{\Gamma, \Sigma, 0}=\left\{g \in G_{0}: g \cdot G_{\Psi \backslash \Gamma, 0}\left(x_{\Gamma, \Sigma}\right)=G_{\Psi \backslash \Gamma, 0}\left(x_{\Gamma, \Sigma}\right)\right\}$, then

(10.10b) $Z_{\Gamma, \Sigma, 0}=\left\{g \in N_{\Gamma, \Sigma, 0}: g\right.$ acts trivially on $\left.G_{\Psi \backslash \Gamma, 0}\left(x_{\Gamma, \Sigma}\right)\right\}$,

(10.10c) $N_{\Gamma, \Sigma, 0}=G_{\Psi \backslash \Gamma, 0} \cdot Z_{\Gamma, \Sigma, 0}$

so $G_{\Psi \backslash \Gamma, 0}$ and $N_{\Gamma, \Sigma, 0}$ have the same action on the holomorphic arc component $G_{\Psi \backslash \Gamma, 0}\left(x_{\Gamma, \Sigma}\right)$ of $G_{0}\left(x_{\Gamma, \Sigma}\right)$.

4. The parabolic subalgebra $\mathfrak{\Re}_{\left[x_{\Gamma}, \Sigma\right]}$ of $\mathcal{G}$, complexification of the Lie algebra of $N_{\Gamma, \Sigma, 0}$, is just the parabolic subalgebra that is the sum of the nonnegative eigenspaces of $\operatorname{ad}\left(\left\{\sum_{\Gamma \backslash \Sigma} x_{\gamma, 0}\right\}-\left\{\sum_{\Gamma \cap \Sigma} x_{\gamma, 0}\right\}\right)$; it is independent of $\Sigma$ if we require that $\Gamma$ and $\Sigma$ be disjoint.

10.11. Corollary. Every $G_{0}$-orbit on $X$ is measurable.

Proof of THEOREM 10.6. If $\varphi$ is a noncompact root then $\mathcal{S}[\varphi]$ denotes the simple algebra $h_{\varphi} \mathbf{C}+g_{\varphi}+\mathcal{G}_{-\varphi}$, and $\mathcal{G}_{0}[\varphi]$ denotes its real form $\mathcal{G}_{0} \cap \mathcal{G}[\varphi] ; G[\varphi]$ and $G_{0}[\varphi]$ are the analytic subgroups for $\mathcal{G}[\varphi]$ and $S_{0}[\varphi]$; and $S[\varphi]$ is the Riemann sphere $G[\varphi]\left(x_{0}\right)$. The $G_{0}[\varphi]$ orbits on $S[\varphi]$ are:

$$
\begin{array}{ll}
\text { lower hemisphere } G_{0}[\varphi]\left(\left\{\exp \frac{\pi}{4} y_{\varphi}\right\}^{n} x_{0}\right), & n \equiv 0 \bmod 4, \\
\text { equator } G_{0}[\varphi]\left(\left\{\exp \frac{\pi}{4} y_{\varphi}\right\}^{n} x_{0}\right), & n \equiv 1 \bmod 2, \\
\text { upper hemisphere } G_{0}[\varphi]\left(\left\{\exp \frac{\pi}{4} y_{\varphi}\right\}^{n} x_{0}\right), & n \equiv 2 \bmod 4 .
\end{array}
$$


Define $g[\Psi]=\sum_{\Psi} g[\psi]$, so the real form $g_{0}[\Psi]=g_{0} \cap g[\Psi]$ is $\sum_{\Psi} g_{0}[\psi]$; then the analytic subgroups for $\mathcal{G}[\Phi]$ and $\mathcal{G}_{0}[\Psi]$ are $G[\Psi]$ $=\prod_{\Psi} G[\psi]$ and $G_{0}[\Psi]=\prod_{\Psi} G_{0}[\psi]$, and $S[\Psi]=G[\Psi]\left(x_{0}\right)$ is the product $\prod_{\Psi} S[\Psi]$ of $|\Psi|$ Riemann spheres. Now the $G_{0}[\Psi]$-orbits on $S[\Psi]$ are the products $\prod_{\Psi} D_{\psi}$ where $D_{\psi}$ is a $G_{0}[\psi]$-orbit on $S[\psi]$. Given such an orbit, define

$$
\begin{aligned}
& \Gamma=\left\{\psi \in \Psi: D_{\psi} \text { is the equator of } S[\psi]\right\}, \text { and } \\
& \Sigma=\left\{\psi \in \Psi: D_{\psi} \text { is the upper hemisphere of } S[\psi]\right\} .
\end{aligned}
$$

Then the orbit is $G_{0}[\Psi]\left(c_{\Gamma} c_{\Sigma}^{2} x_{0}\right)=G_{0}[\Psi]\left(x_{\Gamma, \Sigma}\right)$, and $\Gamma$ and $\Sigma$ are disjoint.

The equator of $S[\psi]$ has descriptions $G_{0}[\psi]\left(c_{\psi} x_{0}\right)=G_{0}[\psi]\left(c_{\psi}^{3} x_{0}\right)$. If $\Gamma$ and $\Sigma$ are arbitrary subsets of $\Psi$, it follows that $x_{\Gamma, \Sigma}=c_{\Gamma \backslash \Sigma} \cdot c_{\Gamma \cap \Sigma}^{3}$ $\cdot c_{\Sigma}^{2} \backslash \Gamma x_{0} \in G_{0}[\Psi]\left(x_{\Gamma, \Sigma \backslash \Gamma}\right)$. Thus

(10.12a) $G_{0}[\Psi]\left(x_{\Gamma, \Sigma}\right)=G_{0}[\Psi]\left(x_{\Gamma, \Sigma \backslash \Gamma}\right)$.

If we also have subsets $\Gamma^{\prime}$ and $\Sigma^{\prime}$ of $\Psi$, it follows that

(10.12b) $G_{0}[\Psi]\left(x_{\Gamma, \Sigma}\right)=G_{0}[\Psi]\left(x_{\Gamma^{\prime}, \Sigma^{\prime}}\right)$ if and only if $(\Sigma \backslash \Gamma)=\left(\Sigma^{\prime} \backslash \Gamma^{\prime}\right)$ and $\Gamma=\Gamma^{\prime}$.

It also follows from $(10.12 \mathrm{a})$ that $G_{0}[\Psi]\left(x_{\Gamma^{\prime}, \Sigma^{\prime}}\right)$ is in the closure of $G_{0}[\Psi]\left(x_{\Gamma, \Sigma}\right)$ if and only if

(i) $\Gamma \subset \Gamma^{\prime}$,

(ii) $(\Sigma \backslash \Gamma) \subset\left(\Sigma^{\prime} \cup \Gamma^{\prime}\right)$, and

(iii) $\{\Psi \backslash(\Sigma \cup \Gamma)\} \subset\left\{\left[\Psi \backslash\left(\Sigma^{\prime} \cup \Gamma^{\prime}\right)\right] \cup \Gamma^{\prime}\right\}$.

Those three conditions can be phrased

(a) $\left(\Sigma^{\prime} \backslash \Gamma^{\prime}\right) \subset(\Sigma \backslash \Gamma)$ and (b) $(\Sigma \cup \Gamma) \subset\left(\Sigma^{\prime} \cup \Gamma^{\prime}\right)$.

Thus

(10.12c) $G_{0}[\Psi]\left(x_{\Gamma^{\prime}, \Sigma^{\prime}}\right)$ is in the ciosure of $G_{0}[\Psi]\left(x_{\Gamma, \Sigma}\right)$

if and only if $\left(\Sigma^{\prime} \backslash \Gamma^{\prime}\right) \subset(\Sigma \backslash \Gamma)$ and $(\Sigma \cup \Gamma) \subset\left(\Sigma^{\prime} \cup \Gamma^{\prime}\right)$.

Finally we observe that

(10.12d) $G_{0}[\Psi]\left(x_{\Gamma, \Sigma}\right)$ is open in $S[\Psi]$ if and only if $\Gamma=\phi$,

(10.12e) $G_{0}[\Psi]\left(x_{\Gamma, \Sigma}\right)$ is closed in $S[\Psi]$ if and only if $\Gamma=\Psi$.

To carry results from $G_{0}[\Psi]$-orbits on $S[\Psi]$ to $G_{0}$-orbits on $X$, we define $A=\exp J a_{u} \subset G_{u}$. As $J \mathfrak{Q}_{u}$ is a maximal abelian subspace of $\mathfrak{T}_{u}$ we have $G_{u}=K A K$. Thus

$$
X=G_{u}\left(x_{0}\right)=K A K\left(x_{0}\right)=K A\left(x_{0}\right)=K \cdot S[\Psi] .
$$

If $x \in X$ we thus have $x=k(s), s \in S[\Psi]$, so $G_{0}(x)=G_{0}(s)=G_{0} \cdot G_{0}[\Psi](s)$. We have disjoint sets $\Gamma$ and $\Sigma$ in $\Psi$ with $G_{0}[\Psi](s)=G_{0}[\Psi]\left(x_{r, \Sigma}\right)$. It follows that $G_{0}(x)=G_{0}\left(x_{r, z}\right)$. That proves part 1 of Theorem 10.6.

Theorem 3.3 says that $X$ has just one closed $G_{0}$-orbit. In [2, Theorem 3.6], $G_{0}\left(x_{\Psi, \phi}\right)$ is shown to be the closed $G_{0}$-orbit on the boundary 
of $X_{0}$ and is identified as the Bergman-Silov boundary of $X_{0}$ in $X$. That proves part 5 of Theorem 10.6.

Let $\Sigma \subset \Psi$. We have $J \subset \mathcal{G}_{0} \cap \odot_{x_{0}}$, and (10.4c) with $\Gamma=\phi$ says $J=\operatorname{ad}\left(c_{\Sigma}^{2}\right) J C \operatorname{ad}\left(c_{\Sigma}^{2}\right) \mathcal{P}_{x_{0}}=\mathcal{P}_{c_{\Sigma}{ }^{2} x_{0}}=\mathcal{P}_{x_{\phi, \Sigma}}$. Thus $\mathcal{g}_{0} \cap \mathcal{P}_{x_{\phi, \Sigma}}$ contains a compact Cartan subalgebra J of $G_{0}$, so Theorem 4.5 says that $G_{0}\left(x_{\phi, \Sigma}\right)$ is open in $X$. Conversely, if $G_{0}\left(x_{\Gamma, \Sigma}\right)$ is open in $X$, then $G_{0}\left(x_{\Gamma, \Sigma}\right) \cap S[\Psi]$ is an open $G_{0}[\Psi]$-invariant subset of $S[\Psi]$ that contains $x_{\Gamma, \Sigma}$. As it is open and $G_{0}[\Psi]$-invariant, it is a union of sets $G_{0}[\Psi]\left(x_{\phi, B}\right), B \subset \Psi$. Thus we have $B \subset \Psi$ with $x_{\Gamma, \Sigma} \in G_{0}[\Psi]\left(x_{\phi, B}\right)$. It follows that the open $G_{0}$-orbits on $X$ are just the $G_{0}\left(x_{\phi, \Sigma}\right)$ with $\Sigma \subset \Psi$.

Let $\Sigma$ and $\Sigma^{\prime}$ be two subsets of $\Psi$. If $\left|\Sigma \cap \Delta^{k}\right|=\left|\Sigma^{\prime} \cap \Delta^{k}\right|$ for $1 \leqq k \leqq p$, then [7, Theorem 2] there is an element $g \in K$ normalizing $J$ such that $\operatorname{ad}(g) \Sigma=\Sigma^{\prime}$, so $G_{0}\left(x_{\phi, \Sigma^{\prime}}\right)=G_{0}\left(c_{\Sigma^{\prime}}^{2} x_{0}\right)=G_{0}\left(g c_{\Sigma}^{2} g^{-1}\right) x_{0}=G_{0}\left(c_{\Sigma}^{2} x_{0}\right)=G_{0}\left(x_{\phi, \Sigma}\right)$. Conversely suppose $G_{0}\left(x_{\phi, \Sigma}\right)=G_{0}\left(x_{\phi, \Sigma^{\prime}}\right)$. Then, as both orbits are open, Theorem 4.9 says that $c_{\Sigma}^{2}$ and $c_{\Sigma^{\prime}}^{2}$ represent the same double coset in a certain space $W_{K} \backslash W_{G}^{3 C_{0}} / W_{P^{\prime} n_{r} P r}^{3 C_{0}}$. There $\mathfrak{F C}_{0}=\mathcal{J}$, so $W_{G}^{3 C_{0}}=W_{G}$; and $P^{r}=\tau P^{r}=K^{c}$, so $W_{P^{r} n_{r P} r}^{3 C_{0}}=W_{K}$. Thus $c_{\Sigma^{\prime}}^{2} \in W_{K} \cdot c_{\Sigma}^{2} \cdot W_{K}$, so we have $w_{i} \in W_{K}$ with $\operatorname{ad}\left(c_{\Sigma^{\prime}}^{2}\right)=w_{1} \cdot \operatorname{ad}\left(c_{\Sigma}^{2}\right) \cdot w_{2}$. If $z$ denotes the central element of $\nVdash$ such that $J=\operatorname{ad}(z) \mid \Re$, and if $\Sigma^{\prime \prime} \subset \Psi$, then $\operatorname{ad}\left(c_{\Sigma^{\prime \prime}}^{2}\right) z-z$ $=\sum_{\psi \in \Sigma^{\prime \prime}}\left(i h_{\psi}\right)$. Thus

$$
\begin{aligned}
-\sum_{\psi \in \Sigma^{\prime}} h_{\psi} & =i\left\{\operatorname{ad}\left(\underset{c_{\Sigma^{\prime}}}{2}\right) z-z\right\}=i\left\{w_{1} \cdot \operatorname{ad}\left(\underset{c_{\Sigma}}{2}\right) \cdot w_{2} z-z\right\} \\
& =i\left\{w_{1} \cdot \operatorname{ad}\left(c_{\Sigma}^{2}\right) z-z\right\}=w_{1} \cdot i\left\{\operatorname{ad}\left(\underset{c_{\Sigma}}{2}\right) z-z\right\} \\
& =w_{1}\left(-\sum_{\psi \in \Sigma} h_{\psi}\right) .
\end{aligned}
$$

As $\frac{1}{2} \sum_{\psi \in \Sigma^{\prime \prime}} x_{\psi, 0}=-(i / 2) \sum_{\psi \in \Sigma^{\prime \prime}} x_{\psi}=\operatorname{ad}\left(c_{\Sigma^{\prime \prime}}\right)\left(-\sum_{\psi \in \Sigma^{\prime \prime}} h_{\psi}\right)$, it follows that $\frac{1}{2} \sum_{\psi \in \Sigma^{\prime}} x_{\psi, 0}$ and $\frac{1}{2} \sum_{\psi \in \Sigma} x_{\psi, 0}$ are conjugate elements of $\mathcal{G}$. Thus the corresponding parabolic subalgebras of $\mathcal{G}$ are conjugate. But [15, Theorem 6.5] those parabolic subalgebras of $G$ are the complex normalizers of the boundary components of types $\Psi \backslash \Sigma$ and $\Psi \backslash \Sigma^{\prime}$ of $X_{0}$ in $X$, so [15, Corollary 6.9] $G_{0}\left(x_{\Sigma, \phi}\right)=G_{0}\left(x_{\Sigma^{\prime}, \phi}\right)$, and it follows [15, Lemma 5.1] that $\left|\Sigma \cap \Delta^{k}\right|=\left|\Sigma^{\prime} \cap \Delta^{k}\right|$ for $1 \leqq k \leqq p$. In conclusion, open orbits $G_{0}\left(x_{\phi, \Sigma}\right)$ and $G_{0}\left(x_{\phi, \Sigma^{\prime}}\right)$ are equal if and only if, for $1 \leqq k \leqq p$, $\left|\Sigma \cap \Delta^{k}\right|=\left|\Sigma^{\prime} \cap \Delta^{k}\right|$. In particular, if $r_{k}=\left|\Psi \cap \Delta^{k}\right|$ then there are precisely $\sum_{1 \leq k \leq p}\left(r_{k}+1\right)=|\Psi|+p$ open $G_{0}$-orbits on $X$. That completes the proof of part 4 of Theorem 10.6.

Consider an open orbit $G_{0}\left(x_{\phi, \Sigma}\right)$. The intersection with $S[\Psi]$ is $G_{0}[\Psi]$-invariant and open in $S[\Psi]$, hence a union of sets $G_{0}[\Psi]\left(x_{\phi, B}\right)$. The sets $B$ there are just the subsets of $\Psi$ such that $G_{0}\left(x_{\phi, \Sigma}\right)=G_{0}\left(x_{\phi, B}\right)$. 


\section{Thus} where

(10.13a) $G_{0}\left(x_{\phi, \Sigma}\right) \cap S[\Psi]=\bigcup_{B} G_{0}[\Psi]\left(x_{\phi, B}\right)$

(10.13b) $B=\left\{B \subset \Psi:\left|B \cap \Delta^{k}\right|=\left|\Sigma \cap \Delta^{k}\right|\right.$ for $\left.1 \leqq k \leqq p\right\}$.

Now consider an orbit $G_{0}\left(x_{\Gamma^{\prime}, \Sigma^{\prime}}\right)$. It is in the closure of $G_{0}\left(x_{\phi, \Sigma}\right)$ if and only if $x_{\Gamma^{\prime}, \Sigma^{\prime}}$ is in that closure. So (10.12c) says that

(10.13c) $G_{0}\left(x_{\Gamma^{\prime}, \Sigma^{\prime}}\right)$ is in the closure of $G_{0}\left(x_{\phi, \Sigma}\right)$ if and only if, for some

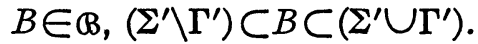

In view of $(10.13 \mathrm{~b})$, we reformulate $(10.13 \mathrm{c})$ as

(10.13d) $G_{0}\left(x_{\Gamma^{\prime}, \Sigma^{\prime}}\right)$ is in the closure of $G_{0}\left(x_{\phi, \Sigma}\right)$ if and only if, for $1 \leqq k \leqq p,\left|\left(\Sigma^{\prime} \backslash \Gamma^{\prime}\right) \cap \Delta^{k}\right| \leqq\left|\Sigma \cap \Delta^{k}\right| \leqq\left(\Sigma^{\prime} \cup \Gamma^{\prime}\right) \cap \Delta^{k} \mid$.

Now consider arbitrary orbits $G_{0}\left(x_{\Gamma, \Sigma}\right)$ and $G_{0}\left(x_{\Gamma^{\prime}, \Sigma^{\prime}}\right)$. According to (10.13d), $G_{0}\left(x_{\Gamma, \Sigma}\right)$ is in the closure of an open orbit $G_{0}\left(x_{\phi, \Sigma^{*}}\right)$ if and only if

$\left|(\Sigma \backslash \Gamma) \cap \Delta^{k}\right| \leqq\left|\Sigma^{*} \cap \Delta^{k}\right| \leqq\left|(\Sigma \cup \Gamma) \cap \Delta^{k}\right| \quad$ for $1 \leqq k \leqq p$.

In particular, $G_{0}\left(x_{\Gamma, \Sigma}\right)$ is in the closures of the open orbits

$$
G_{0}\left(x_{\phi, \Sigma \backslash \Gamma}\right) \text { and } G_{0}\left(x_{\phi, \Sigma \cup \Gamma}\right) \text {. }
$$

If $G_{0}\left(x_{\Gamma^{\prime}, \Sigma^{\prime}}\right)$ is in the closure of $G_{0}\left(x_{\Gamma, \Sigma}\right)$, then it is in the closures of $G_{0}\left(x_{\phi, \Sigma \backslash \Gamma}\right)$ and $G_{0}\left(x_{\phi, \Sigma \cup \Gamma}\right)$, and $(10.13 \mathrm{~d})$ says that $\left|\left(\Sigma^{\prime} \backslash \Gamma^{\prime}\right) \cap \Delta^{k}\right|$ $\leqq\left|(\Sigma \backslash \Gamma) \cap \Delta^{k}\right|$ and $\left|(\Sigma \cup \Gamma) \cap \Delta^{k}\right| \leqq\left|\left(\Sigma^{\prime} \cup \Gamma^{\prime}\right) \cap \Delta^{k}\right|$ for $1 \leqq k \leqq p$. Conversely, if we have those numerical inequalities, then [7, Theorem 2] there is an element $g \in K$ normalizing $\Im$ such that $\operatorname{ad}(g)$ sends $\Sigma^{\prime} \backslash \Gamma^{\prime}$ into $\Sigma \backslash \Gamma$ and sends $\Sigma^{\prime} \cup \Gamma^{\prime}$ to a set containing $\Sigma \cup \Gamma$; as $G_{0}\left(x_{\Gamma^{\prime}, \Sigma^{\prime}}\right)$ $=G_{0}\left(x_{\mathrm{ad}(\theta) \Gamma^{\prime}, \mathrm{ad}(\sigma) \Sigma^{\prime}}\right)$ we thus may assume $\left(\Sigma^{\prime} \backslash \Gamma^{\prime}\right) \subset(\Sigma \backslash \Gamma)$ and $(\Sigma \cup \Gamma)$ $C\left(\Sigma^{\prime} \cup \Gamma^{\prime}\right)$, so $(10.12 \mathrm{c})$ says that $G_{0}\left(x_{\Gamma^{\prime}, \Sigma^{\prime}}\right)$ is in the closure of $G_{0}\left(x_{\Gamma, \Sigma}\right)$. That completes the proof of part 3 of Theorem 10.6.

Orbits $G_{0}\left(x_{\Gamma, \Sigma}\right)$ and $G_{0}\left(x_{\Gamma^{\prime}, \Sigma^{\prime}}\right)$ are equal if and only if each is in the closure of the other. According to part 3 just proved, that can be formulated

$$
\begin{aligned}
\left|\left(\Sigma^{\prime} \backslash \Gamma^{\prime}\right) \cap \Delta^{k}\right| & =\left|(\Sigma \backslash \Gamma) \cap \Delta^{k}\right| \text { and } \\
\left|\left(\Sigma^{\prime} \cup \Gamma^{\prime}\right) \cap \Delta^{k}\right| & =\left|(\Sigma \cup \Gamma) \cap \Delta^{k}\right|,
\end{aligned}
$$

which is equivalent to the criterion

$$
\left|\left(\Sigma^{\prime} \backslash \Gamma^{\prime}\right) \cap \Delta^{k}\right|=\left|(\Sigma \backslash \Gamma) \cap \Delta^{k}\right| \text { and }\left|\Gamma^{\prime} \cap \Delta^{k}\right|=\left|\Gamma \cap \Delta^{k}\right|
$$

asserted in part 2. In particular, if we enumerate $\Psi \cap \Delta^{k}$ $=\left\{\psi_{k, 1}, \cdots, \psi_{k, r_{k}}\right\}$, and the choices of $\Gamma \cap \Delta^{k}$ and $\Sigma \cap \Delta^{k}$ leading to distinct $G_{0}$-orbits are

$$
\begin{aligned}
& \Gamma \cap \Delta^{k}=\left\{\psi_{k, 1}, \cdots, \psi_{k, j}\right\}, \quad 0 \leqq j \leqq r_{k}, \\
& \Sigma \cap \Delta^{k}=\left\{\psi_{k, j+1}, \cdots, \psi_{k, l}\right\}, \quad j \leqq l \leqq r_{k} .
\end{aligned}
$$


The number of such choices is

$$
\begin{aligned}
\sum_{j=0}^{r_{k}}\left(r_{k}+1-j\right) & =\left(r_{k}+1\right)^{2}-\sum_{j=0}^{r_{k}} j=\left(r_{k}+1\right)^{2}-\frac{1}{2} r_{k}\left(r_{k}+1\right) \\
& =\frac{1}{2}\left(r_{k}+1\right)\left(r_{k}+2\right) .
\end{aligned}
$$

Thus there are precisely $\frac{1}{2} \sum_{k=0}^{p}\left(r_{k}+1\right)\left(r_{k}+2\right) G_{0}$-orbits on $X$. That proves part 2 of Theorem 10.6, completing the proof of the theorem.

Q.E.D.

Proof of Theorem 10.7. Decompositions (10.1) and (10.2) show that $\mathfrak{F C}_{\Gamma}$ is an abelian subalgebra of $\mathfrak{K C}_{0}$ with the same dimension as $\mathfrak{J}$. If $\sigma$ is the Cartan involution of $g_{0}$ with fixed point set $\Re$, then $\sigma\left(\mathfrak{H C}_{\Gamma}\right)=\mathfrak{H C}_{\Gamma}$ by $(10.2)$, so $\mathfrak{H C}_{\Gamma}$ is a reductive subalgebra of $\mathcal{G}_{0}$. It follows that $\mathfrak{F C}_{\Gamma}$ is a Cartan subalgebra of $g_{0}$. Note $\mathcal{F C}_{\Gamma}^{C}=\operatorname{ad}\left(c_{\Gamma}\right) \mathfrak{J}^{C}$ $=\operatorname{ad}\left(c_{\Gamma}\right) \operatorname{ad}\left(c_{\Sigma}^{2}\right) \mathcal{J}^{c} C \operatorname{ad}\left(c_{\Gamma} c_{\Sigma}^{2}\right) \mathcal{P}_{x_{0}}=\mathcal{P}_{c_{\Gamma} c_{\Sigma}^{2} x_{0}}=\mathcal{P}_{x_{\Gamma, \Sigma}} ;$ thus $\mathcal{F C}_{\Gamma}$ is a Cartan subalgebra of $\mathcal{S}_{0}$ contained in $\rho_{x_{\Gamma}, \Sigma^{*}}$. It is standard [3] that every Cartan subalgebra of $\mathcal{G}_{0}$ is conjugate to one of the $\mathcal{H C}_{\Gamma}$.

Let $\mathfrak{C}$ be maximally compact among the Cartan subalgebras of $\mathcal{S}_{0}$ contained in $\mathcal{P}_{x_{\Gamma, \Sigma}}$. Conjugating by an element of $G_{0} \cap P_{x_{\Gamma, \Sigma}}$, we may assume

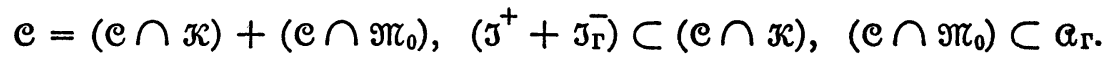

If the latter two inclusions are not equalities, then we have $0 \neq v$ $\in \mathcal{C} \cap J_{\bar{\Gamma} \backslash \Psi}$. Thus $0 \neq v \in \mathcal{P}_{x_{\Gamma}, \Sigma} \cap J_{\Psi} \backslash \bar{\Gamma}$. But ad $\left(c_{\Gamma}^{-1}\right) \operatorname{ad}\left(c_{\Sigma}^{-2}\right)$ sends $\mathcal{P}_{x_{\Gamma, \Sigma}}$ to $P$ and sends $\Im_{\bar{\Psi} \backslash \Gamma}$ to $i Q_{\Gamma}$; thus $0 \neq \operatorname{ad}\left(c_{\Gamma}^{-1}\right) \operatorname{ad}\left(c_{\Sigma^{2}}\right) v \in P \cap i Q_{\Gamma}$, contradicting $P \cap i a_{\Gamma}=0$. Thus

$$
(\mathcal{C} \cap \Re)=\left(J^{+}+\Im_{\Gamma}^{-}\right) \text {and }\left(\mathcal{C} \cap \mathfrak{M}_{0}\right)=a_{\Gamma},
$$

proving $\mathfrak{C}=\mathfrak{F}_{\Gamma}$. Now the first part of Theorem 10.7 is proved.

If $\mathfrak{H}_{\Gamma}$ and $\mathfrak{K}_{\Gamma^{\prime}}$ are conjugate in $\mathcal{G}_{0}$, then for $1 \leqq k \leqq p$ their split parts $a_{\Gamma} \cap g^{k}$ and $a_{r^{\prime}} \cap \mathcal{G}^{k}$ along the simple ideal $g_{0} \cap \mathcal{g}^{k}$ of $g_{0}$ must have the same dimension; those dimensions are $\left|\Gamma \cap \Delta^{k}\right|$ and $\left|\Gamma^{\prime} \cap \Delta^{k}\right|$, which thus must be equal. Conversely, if $\left|\Gamma \cap \Delta^{k}\right|=\left|\Gamma^{\prime} \cap \Delta^{k}\right|$ for $1 \leqq k \leqq p$, then [7, Theorem 2], we have $g \in K$ normalizing $\mathcal{J}$ with ad $(g) \mathcal{H}_{\Gamma^{\prime}}=\mathcal{H C}_{\Gamma}$. That proves part 2 of Theorem 10.7. Parts 4 and 5 are immediate, for $\mathfrak{H C}_{\Gamma}$ is maximally compact if and only if it is conjugate to $\mathfrak{J}=\mathfrak{H C}_{\phi}$, maximally split if and only if it is conjugate to $3^{+}+a_{0}=3 C_{\Psi}$.

If $\mathfrak{H C}_{\Gamma}$ centralizes a $G_{0}$-conjugate $\operatorname{ad}(g) \mathfrak{J}_{\Gamma^{\prime}}$, then $\operatorname{ad}(g) \mathfrak{J}_{\Gamma}^{-} \subset \mathfrak{K C}_{\Gamma}$. As $\mathfrak{K}_{\Gamma}$ is commutative, now $\operatorname{ad}(g){J^{\prime}}_{\Gamma^{\prime}}^{-} \subset \Re^{\prime} \cap \Re_{\Gamma}=J^{+}+J_{\Gamma}^{-} \subset J$, so we can assume that $g \in K$ and $\operatorname{ad}(g) J=3$; thus $a_{\Gamma}+J a_{\Gamma}+\left[a_{\Gamma}, J a_{\Gamma}\right]=g_{0}[\Gamma]$ centralizes $\operatorname{ad}(g) J_{\bar{\Gamma}}^{-}$, so $\mathcal{G}_{0}[\Gamma] \subset \operatorname{ad}(g)\left(\mathcal{G}_{r^{\prime}, 0}\right)$. The former has $\Gamma$, and the latter has $\operatorname{ad}(g) \Gamma^{\prime}$, as maximal set of strongly orthogonal non- 
compact roots. Thus, for $1 \leqq k \leqq p, \quad\left|\Gamma \cap \Delta^{k}\right| \leqq\left|\operatorname{ad}(g) \Gamma^{\prime} \cap \Delta^{k}\right|$ $=\left|\Gamma^{\prime} \cap \Delta^{k}\right|$. Conversely, if $\left|\Gamma \cap \Delta^{k}\right| \leqq\left|\Gamma^{\prime} \cap \Delta^{k}\right|$ for $1 \leqq k \leqq p$, then we conjugate $\Gamma^{\prime}$ by an element of $W$ so that $\Gamma \subset \Gamma^{\prime}$, and then $\mathfrak{H C}_{\Gamma}$ centralizes $\widetilde{T}_{\Gamma^{\prime}}$. That proves part 3 , completing the proof of Theorem 10.7. Q.E.D.

PROOF OF THEOREM 10.9. Our assertions all are known for $G_{0}$-orbits on the boundary of $X_{0}=G_{0}\left(x_{0}\right)$ in $X$ : in Wolf-Koranyi [15] it is shown that $G_{\Psi \backslash \Gamma}\left(x_{0}\right)$ is a compact totally geodesic hermitian symmetric subspace of $X$ (Lemma 4.2); that $G_{\Psi \backslash \Gamma, 0}\left(x_{0}\right) \subset G_{\Psi \backslash \Gamma}\left(x_{0}\right)$ is the Borel embedding of the noncompact dual (Lemma 4.2); that $\Psi \backslash \Gamma$ is a maximal set of strongly orthogonal roots of $\mathcal{S}_{\Psi \backslash \Gamma, 0}$ relative to $I \cap \mathcal{G}_{\Psi \backslash \Gamma, 0}$ (Lemma 4.2); that the holomorphic arc components of the topological boundary $\partial X_{0}$ of $X_{0}$ in $X$ are just the sets $g \cdot c_{\Gamma} G_{\Psi \backslash \Gamma, 0}\left(x_{0}\right)$ with $g \in K$ and $\phi \neq \Gamma \subset \Psi$ (Theorem 4.8); and that the set $B_{\Psi \backslash \Gamma}$ of all elements of $G_{0}$ that preserve $c_{\Gamma} G_{\Psi \backslash \Gamma, 0}\left(x_{0}\right)$ is the parabolic subgroup of $G_{0}$ whose Lie algebra $B_{\Psi \backslash \Gamma}$ is the sum of the nonnegative eigenspaces of $\operatorname{ad}\left(\sum_{\Gamma} x_{\gamma, 0}\right)$ on $\mathcal{G}_{0}$ and satisfies $B_{\Psi \backslash \Gamma}=\mathcal{S}_{\Psi \backslash \Gamma, 0}+Z_{\Psi \backslash \Gamma}$ where $Z_{\Psi \backslash \Gamma}$ is the ideal that is the Lie algebra of the kernel of the action of $B_{\Psi \backslash \Gamma}$ on $c_{\Gamma} G_{\Psi \backslash \Gamma, 0}\left(x_{0}\right)$ (Theorems 6.5 and 6.8 ). Now we make the key observation that every holomorphic arc component of $\partial X_{0}$ is contained in some $G_{0}$-orbit on $\partial X_{0}$, and thus is a holomorphic arc component of that $G_{0}$-orbit. Thus the case $\Sigma=\phi$ of Theorem 10.9, i.e. the case of $G_{0}$-orbits in the closure of $X_{0}$, is contained in the results of WolfKorányi [15].

Now we go to an arbitrary orbit $G_{0}\left(x_{\Gamma, \Sigma}\right)$. To minimize subscripts on subscripts, we adopt the notation

$$
\rho_{\Gamma, \Sigma}=\rho_{x_{\Gamma, \Sigma}} \text { and } \mathfrak{N}_{\Gamma, \Sigma}=\mathfrak{N}_{\left[x_{\Gamma, \Sigma}\right]} \text {. }
$$

Recall the Cartan subalgebra $\mathfrak{H}_{\Gamma} \subset \mathcal{P}_{\Gamma, \Sigma}$ of $\mathcal{G}_{0}$. All roots in this paragraph will be $\mathfrak{H C}_{\Gamma}^{C}$-roots of $\mathrm{g}$. Now

$\Phi_{\Gamma, \Sigma}$ denotes the root system of $\mathcal{P}_{\Gamma, \Sigma}$,

$\Lambda_{\Gamma, \Sigma}$ denotes the root system of $\mathfrak{\Re}_{\Gamma, \Sigma}$.

From (10.3) and (10.4) we have

$$
\operatorname{ad}\left(c_{\Sigma}^{2}\right) \mathfrak{H C}_{\Gamma}=\mathfrak{H C}_{\Gamma} \text { and }\left.\tau \cdot \operatorname{ad}\left(c_{\Sigma}^{2}\right)\right|_{\mathfrak{H C}_{\Gamma}}=\left.\operatorname{ad}\left(c_{\Sigma}^{2}\right) \cdot \tau\right|_{\mathfrak{H C}}
$$

This and $\operatorname{ad}\left(c_{\Sigma}^{2}\right) \mathcal{P}_{\Gamma, \phi}=\mathcal{P}_{\Gamma, \Sigma}$ imply

$$
\operatorname{ad}\left(c_{\Sigma}^{2}\right) \Phi_{\Gamma, \phi}=\Phi_{\Gamma, \Sigma} \quad \text { and } \quad \operatorname{ad}\left(c_{\Sigma}^{2}\right) \tau \Phi_{\Gamma, \phi}=\tau \Phi_{\Gamma, \Sigma} ;
$$

in particular

$$
\operatorname{ad}\left(c_{\Sigma}^{2}\right)\left\{\Phi_{\Gamma, \phi} \cap \tau \Phi_{\Gamma, \phi}\right\}=\Phi_{\Gamma, \Sigma} \cap \tau \Phi_{\Gamma, \Sigma}
$$




$$
\operatorname{ad}\left(c_{\Sigma}^{2}\right)\left\{\Phi_{\Gamma, \phi} \cup \tau \Phi_{\Gamma, \phi}\right\}=\Phi_{\Gamma, \Sigma} \cup \tau \Phi_{\Gamma, \Sigma} .
$$

Recall Theorem 8.5, let $\delta_{r, \Sigma}$ denote the linear form (8.6a) on $\mathcal{F C}_{\Gamma}^{C}$, and let $Q_{\Gamma, \Sigma}$ denote the parabolic subalgebra (8.6c) for $x_{\Gamma, \Sigma}$. Then (10.14a) gives us

$$
\operatorname{ad}\left(c_{\Sigma}^{2}\right) \delta_{\Gamma, \phi}=\operatorname{ad}\left(c_{\Sigma}^{2}\right) \sum_{\Phi_{\Gamma, \phi}^{u} \cap \tau_{\Phi_{\Gamma, \phi}^{u}}^{u}} \varphi=\sum_{\Phi_{\Gamma, \Sigma}^{u} \cap \tau_{\Phi_{\Gamma, \Sigma}^{u}}^{u}} \varphi=\delta_{\Gamma, \Sigma}
$$

so $\operatorname{ad}\left(c_{\Sigma}^{2}\right) Q_{\Gamma, \phi}=Q_{\Gamma, \Sigma}$. Recall Proposition 8.7 and let $\mathfrak{M}_{\Gamma, \Sigma}$ denote the linear space $(8.8 \mathrm{~b})$ for $x_{\Gamma, \Sigma}$. In view of $(10.14 \mathrm{c})$ it follows that $\operatorname{ad}\left(c_{\Sigma}^{2}\right) \mathfrak{M}_{\Gamma, \phi}=\mathfrak{M}_{\Gamma, \Sigma}$. As $G_{0}\left(x_{\Gamma, \phi}\right)$ is partially complex, Theorem 8.9 says $\mathfrak{N}_{\Gamma, \phi}=\mathfrak{M}_{\Gamma, \phi}$, so $\mathfrak{M}_{\Gamma, \phi}$ is an algebra, and thus $\mathfrak{N}_{\Gamma, \Sigma}=\operatorname{ad}\left(c_{\Sigma}^{2}\right) \mathfrak{M}_{\Gamma, \phi}$ is an algebra; now $\Re_{r, \Sigma}=\Re_{r, z}$ by Theorem 8.9. Thus

$$
\mathfrak{\Re}_{\Gamma, \Sigma}=\operatorname{ad}\left(c_{\Sigma}^{2}\right) \Re_{\Gamma, \phi} \text { and } N_{\Gamma, \Sigma}=\operatorname{ad}\left(c_{\Sigma}^{2}\right) N_{\Gamma, \phi}
$$

where the latter are analytic subgroups of $G$. Now let $Z_{r, \Sigma}$ be the kernel of the action of $N_{\Gamma, \Sigma}$ on $N_{\Gamma, \Sigma}\left(x_{\Gamma, \Sigma}\right), Z_{\Gamma, \Sigma}$ its Lie algebra. Then (10.15a) gives us

$$
\mathrm{Z}_{\Gamma, \Sigma}=\operatorname{ad}\left(c_{\Sigma}^{2}\right) \mathrm{Z}_{\Gamma, \phi} \text { and } Z_{\Gamma, \Sigma}=\operatorname{ad}\left(c_{\Sigma}^{2}\right) Z_{\Gamma, \phi}
$$

As (10.4a) says ad $\left(c_{\Sigma}^{2}\right) J_{\bar{\Psi} \backslash \Gamma}^{-}=J_{\bar{\Psi} \backslash \Gamma}^{-},(10.8 \mathrm{a})$ yields

$$
\operatorname{ad}\left(c_{\Sigma}^{2}\right) \mathcal{S}_{\Psi \backslash \Gamma}=\mathcal{S}_{\Psi \backslash \Gamma} .
$$

We already know $\mathfrak{\Re}_{\Gamma, \phi}=\mathcal{S}_{\Psi \backslash \Gamma}+\mathcal{Z}_{\Gamma, \phi}$; thus $(10.15 \mathrm{a}, \mathrm{b}, \mathrm{c})$ combine for (10.15d)

$$
\mathfrak{⿰}_{\Gamma, \Sigma}=\mathcal{S}_{\Psi \backslash \Gamma}+Z_{\Gamma, \Sigma} \text { and } N_{\Gamma, \Sigma}=G_{\Psi \backslash \Gamma} \cdot Z_{\Gamma, \Sigma} .
$$

Now (10.10c) follows. That proves part 3 of Theorem 10.9. As $\mathfrak{N}_{\Gamma, \phi}$ is the sum of the nonnegative eigenspaces of $\operatorname{ad}\left\{\sum_{\Gamma} x_{\gamma, 0}\right\}$ on $\mathcal{G}$, (10.15a) says that $\mathfrak{N}_{r, \Sigma}$ is the sum of the nonnegative eigenspaces of

$$
\operatorname{ad}\left\{\operatorname{ad}\left(c_{\Sigma}^{2}\right) \sum_{\Gamma} x_{\gamma, 0}\right\}=\operatorname{ad}\left(\left\{\sum_{\Gamma \backslash \Sigma} x_{\gamma, 0}\right\}-\left\{\sum_{\Gamma \cap \Sigma} x_{\gamma, 0}\right\}\right) .
$$

That proves part 4 of Theorem 10.9 .

Note that $c_{\Gamma}$ centralizes $G_{\Psi} \backslash \Gamma$. Using $(10.15 \mathrm{c})$, we have

$$
G_{\Psi \backslash \Gamma}\left(x_{\Gamma, \Sigma}\right)=c_{\Sigma}^{2} G_{\Psi \backslash \Gamma}\left(x_{\Gamma, \phi}\right)=c_{\Gamma} c_{\Sigma}^{2} G_{\Psi \backslash \Gamma}\left(x_{0}\right) .
$$

We already know that $G_{\Psi \backslash \Gamma}\left(x_{\Gamma, \phi}\right)$ is a compact totally geodesic hermitian symmetric subspace of $X$, rank $|\Psi \backslash \Gamma|$ with $\Psi \backslash \Gamma$ as maximal set of strongly orthogonal noncompact roots of $\mathcal{S}_{\Psi \backslash \Gamma, 0}$ relative to $I \cap \mathcal{G}_{\Psi \backslash \Gamma, 0}$. Now the same statements follow for $G_{\Psi \backslash \Gamma}\left(x_{\Gamma, \Sigma}\right)$. That proves part 1 of Theorem 10.9 . 
$G_{\Psi \backslash \Gamma, 0}\left(x_{\Gamma, \Sigma}\right)$ is open in $G_{\Psi \backslash \Gamma}\left(x_{\Gamma, \Sigma}\right)$ by $(10.15 \mathrm{~d})$ and the fact that $G_{0}\left(x_{\Gamma, \Sigma}\right)$ is partially complex. $G_{\Psi \backslash \Gamma, 0}\left(x_{\Gamma, \Sigma}\right)$ is symmetric with invariant kaehler metric by Corollary 9.25. If $\Gamma \cap \Sigma=\phi$ and $\Sigma$ is a (possibly empty) union of sets $(\Psi \backslash \Gamma) \cap \Delta^{k}$ for which $G_{0}^{k}\left(x_{0}\right)$ is of tube type, then $G_{\Psi \backslash \Gamma, 0}$ has the same (thus compact) isotropy subgroups at $x_{\Gamma, \phi}$ and $x_{\Gamma, \Sigma}$, so the metric is positive definite. If $\Gamma \cap \Psi=\phi$ and the metric is positive definite, then $G_{\Psi \backslash \Gamma, 0}$ has the same isotropy subgroups at $x_{\Gamma, \phi}$ and $x_{\Gamma, \Sigma}$, so [15, Lemma 5.6] the (-1)-eigenspace of $\operatorname{ad}\left(c_{\Sigma}^{4}\right)$ on $\mathcal{S}_{\Psi \backslash \Gamma}$ is zero, and the last statement of [15, Theorem 5.7] says that $\Sigma$ is a union of sets $(\Psi \backslash \Gamma) \cap \Delta^{k}$ for which $G_{\Psi \backslash \Gamma, 0}^{k}\left(x_{0}\right)$ is of tube type. However, [15, Corollary 4.11] says that the latter is equivalent to $G_{0}^{k}\left(x_{0}\right)$ being of tube type. That proves part 2, completing the proof of Theorem 10.9. Q.E.D.

11. Hermitian symmetric spaces: compact subvarieties and Siegel domain realizations. Retain the notation and terminology of $\$ 10$. We will apply our explicit analysis of the $G_{0}$-orbit structure of $X$ to obtain certain "generalized Siegel domain" realizations of an arbitrary orbit $G_{0}\left(x_{\Gamma, \Sigma}\right) \subset X$. Those realizations are composed of several decompositions of $G_{0}\left(x_{\Gamma, \Sigma}\right)$ as follows.

1. $\alpha: G_{0}\left(x_{\Gamma, \Sigma}\right) \rightarrow G_{0} / N_{\Gamma, \Sigma, 0}$ is the $K$-equivariant fibration whose fibres are the holomorphic arc components $k \cdot G_{\Psi \backslash \Gamma, 0}\left(x_{\Gamma, \Sigma}\right)$ $=k c_{\Gamma} G_{\Psi \backslash \Gamma, 0}\left(x_{\phi, \Sigma}\right), k \in K$, of $G_{0}\left(x_{\Gamma, \Sigma}\right)$, and whose base is the space $G_{0} / N_{\Gamma, \Sigma, 0}=K /\left(K \cap N_{\Gamma, \Sigma, 0}\right)$ of holomorphic arc components of $G_{0}\left(x_{\Gamma, \Sigma}\right)$.

2. $\beta: G_{0}\left(x_{\Gamma, \Sigma}\right) \rightarrow K\left(x_{\Gamma, \Sigma}\right)$ is a $K$-equivariant fibration whose restriction $\beta_{k}$ to a holomorphic arc component $k \cdot G_{\Psi \backslash \Gamma, 0}\left(x_{\Gamma, \Sigma}\right)$ is a holomorphic fibration of that arc component over its maximal compact subvariety $k \cdot\left(K \cap G_{\Psi \backslash \Gamma, 0}\right)\left(x_{\Gamma, \Sigma}\right)$. Assume $\Gamma$ and $\Sigma$ disjoint. Then the fibre of $\beta$ (and also of $\beta_{k}$ ) over $k k^{\prime}\left(x_{\Gamma, \Sigma}\right), k^{\prime} \in K \cap G_{\Psi \backslash \Gamma, 0}$, is a certain hermitian symmetric space

$$
\begin{aligned}
k k^{\prime} \cdot G_{\Psi \backslash \Gamma, 0}^{\Sigma}\left(x_{\Gamma, \Sigma}\right) & =k k^{\prime} c_{\Gamma} c_{\Sigma}^{2} \cdot G_{\Psi \backslash \Gamma, 0}^{\Sigma}\left(x_{0}\right) \\
= & k k^{\prime} c_{\Gamma} c_{\Sigma \Sigma}^{2}\left\{G_{\Psi \backslash(\Gamma \cup \Sigma), 0}\left(x_{0}\right) \times G_{\Sigma, 0}^{\Sigma}\left(x_{0}\right)\right\}
\end{aligned}
$$

of noncompact type and rank $|\Psi \backslash \Gamma|$ where $G_{\Sigma, 0}^{\Sigma}\left(x_{0}\right)$ denotes the tube domain part (c.f. [2]) of the domain $G_{\Sigma, 0}\left(x_{0}\right) \cdot \alpha$ factors through $\beta$, i.e. $\alpha \cdot \beta^{-1}: K\left(x_{\Gamma, \Sigma}\right) \rightarrow G_{0} / N_{\Gamma, \Sigma, 0}$ is a well-defined $K$-equivariant fibration.

3. $\gamma^{A, B}: G_{0}\left(x_{\Gamma, \Sigma}\right) \rightarrow W \cdot G_{E^{\prime}, 0}^{B^{\prime}}\left(x_{\Gamma, \Sigma}\right)$ where $W$ is a certain subset of $K$ and we have disjoint decompositions

$\Sigma=A \cup A^{\prime}, \quad \Psi \backslash(\Gamma \cup \Sigma)=B \cup B^{\prime}, \quad E=A \cup B, \quad E^{\prime}=A^{\prime} \cup B^{\prime}$. 
Given $k \in W$, the restriction $\gamma_{i}^{A, B}$ of $\gamma^{A, B}$ to $\left(\gamma^{A, B}\right)^{-1}\left(k \cdot G_{E^{\prime}, 0}^{B^{\prime}}\left(x_{\Gamma, \Sigma}\right)\right)$ is a holomorphic fibration $\left.\gamma_{k}^{A, B}: k \cdot G_{\Psi, 0}^{\Sigma} \backslash \gamma_{\Gamma, \Sigma}\right) \rightarrow k \cdot G_{E^{\prime}, 0}^{B^{\prime}}\left(x_{\Gamma, \Sigma}\right)$ equivalent to a certain projection that is implicit (but made explicit in the appendix to this \$11) in the intrinsic realization (c.f. [15] or the appendix to this $\$ 11)$ of $G_{\Psi, \Gamma, 0}^{\Sigma}\left(x_{0}\right)$ as a Siegel domain of type III over its boundary component $G_{E^{\prime}, 0}^{B^{\prime}}\left(x_{E, \phi}\right) \cdot \gamma^{A, B}$ is not necessarily continuous, but it is real analytic almost everywhere, and $\beta$ factors through $\gamma^{A, B}$.

The fibration $\alpha: G_{0}\left(x_{\Gamma, \Sigma}\right) \rightarrow G_{0} / N_{\Gamma, \Sigma, 0}$ is the subject of Theorem 8.15 with details supplied by Theorem 10.9. The fibration $\beta: G_{0}\left(x_{\Gamma, \Sigma}\right)$ $\rightarrow K\left(x_{\Gamma, \Sigma}\right)$ is described in Theorem 11.8 below, while $\gamma^{A, B}$ is described by Proposition 11.20 and Theorem 11.23. Proposition 11.20 is a transcription of Proposition 11.3A (A for appendix) which extends the Wolf-Korányi description [15] of the realizations of hermitian symmetric spaces as Siegel domains of type III.

Let $\Gamma$ and $\Sigma$ be disjoint subsets of $\Psi$. We decompose the real form $\mathcal{S}_{\Psi \backslash \Gamma, 0}=\mathcal{S}_{0} \cap \mathcal{G}_{\Psi \backslash \Gamma}$ of $\mathcal{S}_{\Psi \backslash \Gamma}$ by

$$
\begin{aligned}
& \text { (11.1a) } \quad S_{\Psi \backslash \Gamma, 0}=K_{\Psi \backslash \Gamma}+\mathscr{K}_{\Psi \backslash \Gamma, 0}, \quad K_{\Psi \backslash \Gamma}=K \cap \mathcal{S}_{\Psi \backslash \Gamma} \quad \text { and } \\
& \mathfrak{N}_{\Psi \backslash \Gamma, 0}=\mathfrak{T}_{0} \cap \mathcal{G}_{\Psi \backslash \Gamma} .
\end{aligned}
$$

If $\psi \in \Psi$ then $\operatorname{ad}\left(c_{\psi}\right)$ has order 4 on the odd dimensional irreducible representation spaces of $\operatorname{ad}_{\mathcal{g}}(g[\psi])$, order 8 on the even dimensional ones; so $\operatorname{ad}\left(c_{\psi}^{4}\right)$ has square 1 . Now

$$
\operatorname{ad}\left(c_{\psi}^{4}\right)=\operatorname{ad}\left(c_{\psi}^{4}\right)^{-1}=\tau^{-1} \cdot \operatorname{ad}\left(c_{\psi}^{4}\right) \cdot \tau
$$

shows that $\operatorname{ad}\left(c_{\psi}^{4}\right)$ preserves $G_{0}$, and

$$
\operatorname{ad}\left(c_{\psi}^{4}\right)=\operatorname{ad}\left(c_{\psi}^{4}\right)^{-1}=\sigma^{-1} \cdot \operatorname{ad}\left(c_{\psi}^{4}\right) \cdot \sigma
$$

shows further that $\operatorname{ad}\left(c_{\psi}^{4}\right)$ preserves $\nVdash$ and $\Re_{0}$. Define

(11.1b) $\mathcal{S}_{\Psi \backslash \Gamma}^{\Sigma}=\left\{v \in \mathcal{S}_{\Psi \backslash \Gamma}: \operatorname{ad}\left(c_{\Sigma}^{4}\right) v=v\right\}$ and $\mathcal{S}_{\Psi \backslash \Gamma, 0}^{\Sigma}=\mathcal{S}_{0} \cap \mathcal{S}_{\Psi \backslash \Gamma}^{\Sigma}$.

Now $\mathcal{G}_{\Psi \backslash \Gamma, 0}^{\Sigma}$ is a real form of $\mathcal{G}_{\Psi \backslash \Gamma}^{\Sigma}$, and in fact

$$
\begin{aligned}
\mathcal{S}_{\Psi \backslash \Gamma, 0}^{\Sigma} & =K_{\Psi \backslash \Gamma}^{\Sigma}+\Re_{\Psi \backslash \Gamma, 0}^{\Sigma}, \quad K_{\Psi \backslash \Gamma}^{\Sigma}=\nVdash \cap g_{\Psi \backslash \Gamma}^{\Sigma} \quad \text { and } \\
\mathfrak{T}_{\Psi \backslash \Gamma, 0}^{\Sigma} & =\Re_{0} \cap \oint_{\Psi \backslash \Gamma .}^{\Sigma} .
\end{aligned}
$$

Finally, we define

$$
\begin{aligned}
& \text { (11.1d) } Q_{\Psi \backslash \Gamma}^{\Sigma} \text { is the }(-1) \text {-eigenspace of } \operatorname{ad}\left(c_{\Sigma}^{4}\right) \text { on } \mathcal{K}_{\Psi \backslash \Gamma}, \\
& \text { (11.1e) } \mathcal{R}_{\Psi \backslash \Gamma, 0}^{\Sigma} \text { is the (-1)-eigenspace of } \operatorname{ad}\left(c_{\Sigma}^{4}\right) \text { on } \Re_{\Psi \backslash \Gamma, 0} .
\end{aligned}
$$


That gives us symmetric decompositions under $\operatorname{ad}\left(c_{\Sigma}^{4}\right)$ :

$$
\mathcal{K}_{\Psi \backslash \Gamma}=\mathcal{K}_{\Psi \backslash \Gamma}^{\Sigma}+Q_{\Psi \backslash \Gamma}^{\Sigma} \text { and } S_{\Psi \backslash \Gamma, 0}=S_{\Psi \backslash \Gamma, 0}^{\Sigma}+\left(Q_{\Psi \backslash \Gamma}^{\Sigma}+Q_{\Psi \backslash \Gamma, 0}^{\Sigma}\right) \text {. }
$$

Roman letters denote corresponding analytic groups.

We collect some information in a sequence of lemmas.

11.2. Lemma. $K_{\Psi \backslash \Gamma}=K \cap G_{\Psi \backslash \Gamma, 0}$, maximal compact subgroup of $G_{\Psi \backslash \Gamma, 0}$.

11.3. LemMa. $K_{\Psi \backslash \Gamma}^{\Sigma}=K \cap G_{\Psi \backslash \Gamma, 0}^{\Sigma}$, maximal compact subgroup of $G_{\Psi \backslash \Gamma, 0 .}^{\Sigma}$. Both $K_{\Psi \backslash \Gamma}^{\Sigma}$ and $G_{\Psi \backslash \Gamma, 0}^{\Sigma}$ are normalized by $c_{\Gamma} c_{\Sigma}^{2}$, and $K_{\Psi \backslash \Gamma}^{\Sigma}$ is the isotropy subgroup of $G_{\Gamma \backslash \Psi, 0}^{\mathrm{\Sigma}}$ both at $x_{0}$ and at $x_{\Gamma, \Sigma}$. In particular

$$
G_{\Psi \backslash \Gamma, 0}^{\Sigma}\left(x_{\Gamma, \Sigma}\right)=c_{\Gamma} c_{\Sigma}^{2} G_{\Psi \backslash \Gamma, 0}^{\Sigma}\left(x_{0}\right) \cong G_{\Psi \backslash \Gamma, 0}^{\Sigma} / K_{\Psi \backslash \Gamma}^{\Sigma},
$$

and that orbit is a totally geodesic hermitian symmetric subspace of noncompact type and rank $|\Psi \backslash \Gamma|$ in $c_{\Gamma} c_{\Sigma}^{2} X_{0}$.

11.4. Lemma. $K_{\Psi \backslash \Gamma}^{\Sigma}$ is the isotropy subgroup of $K_{\Psi \backslash \Gamma}$ at $x_{\Gamma, \Sigma}$, and the orbit $K_{\Psi \backslash \Gamma}\left(x_{\Gamma, \Sigma}\right) \cong K_{\Psi \backslash \Gamma} / K_{\Psi \backslash \Gamma}^{\Sigma}$ is a totally geodesic hermitian symmetric subspace of compact type in $X$.

11.5. LemMa. The isotropy subgroup of $G_{\Psi \backslash \Gamma, 0}$ at $x_{\Gamma, \Sigma}$ is the analytic subgroup with Lie algebra $\mathcal{K}_{\Psi \backslash \Gamma}^{\Sigma}+R_{\Psi \backslash \Gamma, 0}^{\Sigma}=\operatorname{ad}\left(c_{\Gamma} c_{\Sigma}^{2}\right) \mathcal{K}_{\Psi \backslash \Gamma}^{C} \cap \mathcal{G}_{\Psi \backslash \Gamma, 0}$.

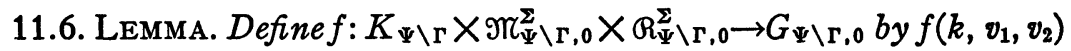
$=k \cdot \exp v_{1} \cdot \exp v_{2}$. Then $f$ is a diffeomorphism.

Proof of Lemmas. $G_{\Psi \backslash \Gamma, 0}\left(x_{0}\right) \cong G_{\Psi \backslash \Gamma, 0} /\left(K \cap G_{\Psi \backslash \Gamma, 0}\right)$ is a hermitian symmetric subspace of noncompact type and rank $|\Psi \backslash \Gamma|$ in $X_{0}$. Thus $K \cap G_{\Psi \backslash \Gamma, 0}$ is connected and maximal compact in $G_{\Psi \backslash \Gamma, 0}$. As $K \cap G_{\Psi \backslash \Gamma, 0}$ has Lie algebra $\varkappa_{\Psi \backslash \Gamma}$, now $K \cap G_{\Psi \backslash \Gamma, 0}=K_{\Psi \backslash \Gamma}$ and Lemma 11.2 is proved.

Note $a_{\Psi \backslash \Gamma, 0} \subset \mathcal{G}_{\Psi \backslash \Gamma, 0}^{\Sigma} \subset \mathcal{G}_{\Psi \backslash \Gamma}$ and $\operatorname{ad}(z) \mathscr{T} \sum_{\Psi \backslash \Gamma, 0}^{\Sigma}=\Re_{\Psi \backslash \Gamma, 0}^{\Sigma}$. It follows that $G_{\Psi}^{\Sigma} \backslash \mathrm{r}, 0\left(x_{0}\right)$ is a totally geodesic hermitian symmetric submanifold of noncompact type and rank $|\Psi \backslash \Gamma|$ in $X_{0}$. As above, now $K_{\Psi \backslash \Gamma}^{\Sigma}$ $=K \cap G_{\Psi}^{\Sigma} \backslash \Gamma, 0$ isotropy subgroup at $x_{0}$ and maximal compact subgroup of $G_{\Psi}^{\Sigma} \backslash \Gamma, 0$.

As $c_{\Gamma}$ centralizes $G_{\Psi \backslash \Gamma}$ it centralizes the subgroups $K_{\Psi \backslash \Gamma}^{\Sigma}$ and $G_{\Psi \backslash \Gamma, 0}^{\Sigma}$. As $\operatorname{ad}\left(c_{\Sigma}^{2}\right)$ preserves $\mathcal{S}_{\Psi} \backslash \Gamma$ and commutes with $\operatorname{ad}\left(c_{\Sigma}^{4}\right)$, it preserves $\mathcal{S}_{\Psi}^{\Sigma} \backslash \Gamma$. Now $\operatorname{ad}\left(c_{\Sigma}^{2}\right)$ preserves $\mathcal{S}_{\Psi}^{\Sigma} \backslash \Gamma, 0$ because

$$
\operatorname{ad}\left(c_{\Sigma}^{2}\right)=\operatorname{ad}\left(c_{\Sigma}^{2}\right)^{-1}=\tau^{-1} \cdot \operatorname{ad}\left(c_{\Sigma}^{2}\right) \cdot \tau \quad \text { on } \oint_{\Psi \backslash \Gamma}^{\Sigma} .
$$

Furthermore

$$
\operatorname{ad}\left(c_{\Sigma}^{2}\right)=\operatorname{ad}\left(c_{\Sigma}^{2}\right)^{-1}=\sigma^{-1} \cdot \operatorname{ad}\left(c_{\Sigma}^{2}\right) \cdot \sigma \quad \text { on } S_{\Psi \backslash \Gamma, 0}^{\Sigma}
$$




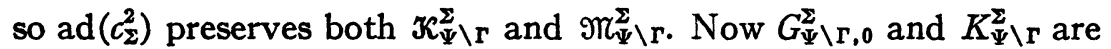
centralized by $\operatorname{ad}\left(c_{\Gamma}\right)$ and normalized by $\operatorname{ad}\left(c_{\Sigma}^{2}\right)$, so they are normalized by $\operatorname{ad}\left(c_{\Gamma} c_{\Sigma}^{2}\right)$. As $K_{\Psi \backslash \Gamma}^{\Sigma}$ is the isotropy subgroup of $G_{\Psi \backslash \Gamma, 0}^{\Sigma}$ at $x_{0}$, it thus is the isotropy subgroup at $c_{\Gamma} c_{\Sigma}^{2} x_{0}=x_{r, \Sigma}$. Lemma 11.3 is proved.

The symmetry of $X$ at $x_{\Gamma, \Sigma}$ is $\operatorname{ad}\left(c_{\Gamma} c_{\Sigma}^{2}\right) \cdot \sigma \cdot \operatorname{ad}\left(c_{\Gamma} c_{\Sigma}^{2}\right)^{-1}=\operatorname{ad}\left(c_{\Gamma} c_{\Sigma}^{2}\right)^{2} \cdot \sigma$ $=\operatorname{ad}\left(c_{\Gamma}^{2}\right) \cdot \operatorname{ad}\left(c_{\Sigma}^{4}\right) \cdot \sigma$. It acts on $G_{\Psi \backslash \Gamma, 0}$ as ad $\left(c_{\Sigma}^{4}\right) \cdot \sigma$. Thus it normalizes $K_{\Psi \backslash \Gamma}$, so $K_{\Psi \backslash \Gamma}\left(x_{\Gamma, \Sigma}\right)$ is a compact totally geodesic symmetric subspace of $X$. It is a complex submanifold of $X$ by Lemma 5.1, so it is hermitian symmetric. The isotropy subgroup of $K_{\Psi \backslash \Gamma}$ at $x_{\Gamma, \Sigma}$ contains $K_{\Psi \backslash \Gamma}^{\Sigma}$, has Lie algebra $\mathfrak{K}_{\Psi \backslash \Gamma}^{\Sigma}$ that contains the center of $\mathcal{K}_{\Psi \backslash \Gamma}$; thus $K_{\Psi \backslash \mathrm{r}}^{\Sigma}\left(x_{0}\right)$ is of compact type and necessarily simply connected; now $K_{\Psi \backslash \Gamma}^{\Sigma}$ is the isotropy subgroup of $K_{\Psi \backslash \Gamma}$ at $x_{\Gamma, \Sigma}$. Lemma 11.4 is proved.

The isotropy subalgebra of $\mathcal{S}_{\Psi \backslash \Gamma, 0}$ at $c_{\Gamma} x_{0}$ is $\varkappa_{\Psi \backslash \Gamma}$, so the isotropy subalgebra at $c_{\Sigma}^{2}\left(c_{\Gamma} x_{0}\right)=x_{\Gamma, \Sigma}$ is ad $\left(c_{\Sigma}^{2}\right) \pi_{\Psi \backslash \Gamma}^{C} \cap g_{\Psi \backslash \Gamma, 0}$. Now ad $\left(c_{\Sigma}^{2}\right)$ preserves $\mathcal{K}_{\Psi \backslash \Gamma}^{\Sigma}$, as seen above, and sends $Q_{\Psi \backslash \Gamma}^{\Sigma}$ to $i \mathcal{Q}_{\Psi \backslash \Gamma}^{\Sigma}$ by [15, Lemma 5.6]. Thus $\mathcal{S}_{\Psi \backslash \Gamma, 0}$ has isotropy subalgebra $\kappa_{\Psi \backslash \Gamma}^{\Sigma}+\Re_{\Psi \backslash \Gamma, 0}^{\Sigma}$ at $x_{\Gamma, \Sigma}$. The isotropy subgroup of $G_{\Psi \backslash \Gamma, 0}$ at $x_{\Gamma, \Sigma}$ is connected by Theorem 5.4, so it must be the analytic subgroup for $\mathcal{K}_{\Psi \backslash \Gamma}^{\Sigma}+\Omega_{\Psi \backslash \Gamma, 0}^{\Sigma}$. Lemma 11.5 is proved.

Define $\varphi: \Re_{\Psi \backslash \Gamma, 0} \rightarrow G_{\Psi \backslash \Gamma, 0}\left(x_{0}\right)$ by $\varphi(v)=\exp \left(-v_{2}\right) \cdot \exp \left(-v_{1}\right) \cdot x_{0}$ where $v=v_{1}+v_{2}, v_{1} \in \mathfrak{N}_{\Psi \backslash \Gamma, 0}^{\Sigma}$ and $v_{2} \in \mathcal{Q}_{\Psi}^{\Sigma} \backslash \Gamma, 0$. We view $\varphi$ as a differentiable map from the euclidean space $\Re_{\Psi \backslash \Gamma, 0}$ to the symmetric space $G_{\Psi \backslash \Gamma, 0}\left(x_{0}\right)$ of noncompact type. We will prove $\left\|\varphi_{*} \xi\right\| \geqq\|\xi\|$ for every tangent vector $\xi$ to $\mathfrak{T}_{\Psi \backslash \Gamma, 0}$. Then [13, Theorem 1] $\varphi$ is surjective and covering, hence a diffeomorphism because $G_{\Psi \backslash \Gamma, 0}\left(x_{0}\right)$ is simply connected, and it follows that from $f\left(k, v_{1}, v_{2}\right)^{-1} x_{0}=\varphi\left(v_{1}+v_{2}\right)$ that $f$ defines a diffeomorphism of $K \times \Re_{\Psi \backslash \Gamma, 0}^{\Sigma} \times \mathcal{R}_{\Psi \backslash \Sigma, 0}^{\Sigma}$ onto $G_{\Psi \backslash \Gamma, 0}$.

Let $v_{1} \in \Re_{\Psi}^{\Sigma} \backslash \mathrm{r}, 0$ be fixed, $v_{2} \in \mathcal{R}_{\Psi, \Gamma, 0}^{\Sigma}$ variable. Then $\varphi\left(v_{1}+v_{2}\right)$ $=\exp \left(-v_{2}\right) \exp \left(-v_{1}\right) x_{0}=\exp \left(-v_{1}\right) \cdot \exp \left(v_{1}\right) \cdot \exp \left(-v_{2}\right) \cdot \exp \left(-v_{1}\right) x_{0}$ $=\exp \left(-v_{1}\right) \cdot\left\{\operatorname{ad}\left(\exp v_{1}\right) \cdot \exp \left(-v_{2}\right)\right\} x_{0}=\exp \left(-v_{1}\right) \cdot\left\{\exp \left(-\operatorname{ad}\left(\exp v_{1}\right)\right.\right.$ $\left.\left.\cdot v_{2}\right)\right\} x_{0}$. Now $\exp \left(-v_{1}\right)$ is an isometry on $G_{\Psi \backslash \Gamma, 0}\left(x_{0}\right)$, and

$$
\operatorname{ad}\left(\exp v_{1}\right) \cdot v_{2}=\exp \left(\operatorname{ad} v_{1}\right) \cdot v_{2}=\sum_{n \geq 0} \frac{1}{n !} \operatorname{ad}\left(v_{1}\right)^{n} \cdot v_{2} .
$$

Note $\operatorname{ad}\left(v_{1}\right)^{n} v_{2} \in \mathcal{R}_{\Psi}^{\Sigma} \backslash \Gamma, 0$ for $n$ even, $\in Q_{\Psi \backslash \Gamma}^{\Sigma}$ for $n$ odd; so

$$
\operatorname{ad}\left(\exp v_{1}\right) \cdot v_{2}=\sum_{n \geq 0} \frac{1}{(2 n) !} \operatorname{ad}\left(v_{1}\right)^{2 n} \cdot v_{2}+\sum_{n \geq 0} \frac{1}{(2 n+1) !} \operatorname{ad}\left(v_{1}\right)^{2 n+1} \cdot v_{2}
$$

with the first sum in $Q_{\Psi}^{\Sigma} \backslash \Gamma, 0$ and the second in $Q_{\Psi}^{\Sigma} \backslash \Gamma$. As ad $\left(v_{1}\right)$ is symmetric, so $\operatorname{ad}\left(v_{1}\right)^{2}$ is positive semidefinite, now 


$$
\|\left(\operatorname{ad}\left(\exp v_{1}\right) \cdot v_{2}\right) \Re\left(\Psi \backslash r, 0\|\geqq\| v_{2} \|\right. \text {. }
$$

Thus $\varphi$ does not decrease lengths of tangent vectors on $v_{1}+R_{\Psi \backslash r, 0}^{\Sigma}$.

Let $v_{2} \in \mathcal{R}_{\Psi}^{\Sigma} \backslash \Gamma, 0$ be fixed, $v_{1} \in \mathfrak{M}_{\Psi \backslash \Gamma, 0}^{\Sigma}$ variable. Then $\varphi\left(v_{1}+v_{2}\right)$ $=\exp \left(-v_{2}\right) \exp \left(-v_{1}\right) x_{0}$ is the composition of -1 on $\mathfrak{T}_{\Psi \backslash \Gamma, 0}^{\Sigma}$, exponential map in the symmetric space $G_{\Psi \backslash \Gamma, 0}^{\Sigma}\left(x_{0}\right)$ of noncompact type, and left translation by $\exp \left(-v_{2}\right)$. As $G_{\Psi, \Gamma, 0}^{\sum}\left(x_{0}\right)$ has curvature $\leqq 0$, now $\varphi$ does not decrease lengths of tangent vectors to $\mathfrak{M}_{\Psi \backslash \Gamma, 0}^{\Sigma}+v_{2}$.

Fix $v_{1} \in \mathfrak{M}_{\Psi \backslash \Gamma, 0}^{\Sigma}$ and let $w_{1}$ be variable there; fix $v_{2} \in \mathscr{Q}_{\Psi \backslash \Gamma, 0}^{\Sigma} \backslash$ and let $w_{2}$ be variable there. Now

$$
\begin{aligned}
\varphi\left(v_{1}+R_{\Psi \backslash \Gamma, 0}^{\Sigma}\right) & =\left\{\exp \left(-v_{2}-w_{2}\right) \cdot \exp \left(-v_{1}\right) \cdot x_{0}\right\}, \\
\varphi\left(\Re_{\Psi \backslash \Gamma, 0}^{\Sigma}+v_{2}\right) & =\left\{\exp \left(-v_{2}\right) \cdot \exp \left(-v_{1}-w_{1}\right) \cdot x_{0}\right\} .
\end{aligned}
$$

Those manifolds intersect at $\varphi\left(v_{1}+v_{2}\right)=\exp \left(-v_{2}\right) \cdot \exp \left(-v_{1}\right) \cdot x_{0}$, and we want to show the intersection there to be orthogonal. For that, translate by the isometry $\exp \left(v_{1}\right)$ sending $\exp \left(-v_{2}\right) \exp \left(-v_{1}\right) x_{0}$ to $\exp \left(-\operatorname{ad}\left(\exp v_{1}\right) \cdot v_{2}\right) x_{0} \in \varphi\left(\mathcal{Q}_{\Psi}^{\Sigma} \backslash \Gamma, 0\right)$, and note first that $\exp \left(v_{1}\right) \varphi\left(\mathcal{Q}_{\Psi}^{\Sigma} \backslash \Gamma, 0\right)$ consists of all

$$
\exp \left(-\operatorname{ad}\left(\exp v_{1}\right) \cdot v_{2}-\operatorname{ad}\left(\exp v_{1}\right) w_{2}\right) \cdot x_{0}
$$

and second that $\exp \left(v_{1}\right) \varphi\left(\mathscr{T}_{\Psi \backslash \Gamma, 0}^{\mathbb{\Sigma}}\right)$ consists of all

$$
\exp \left(-\operatorname{ad}\left(\exp v_{1}\right) \cdot v_{2}\right) \exp \left(-w_{1}\right) \cdot x_{0} .
$$

Thus the question of orthogonality is reduced to the case $v_{1}=0$, where it follows from first

$$
\exp \left(v_{2}\right) \varphi\left(\boldsymbol{R}_{\Psi \backslash \Gamma, 0}^{\Sigma}\right)=\left\{\exp \left(-w_{2}\right) x_{0}\right\}=\varphi\left(Q_{\Psi \backslash \Gamma, 0}^{\Sigma}\right)
$$

because $\exp \left(v_{2}\right) \exp \left(-v_{2}-w_{2}\right) \equiv \exp \left(-w_{2}\right) \bmod K_{\Psi \backslash \Gamma}^{\Sigma}$, and second from

$$
\exp \left(v_{2}\right) \varphi\left(\Re_{\Psi \backslash \Gamma, 0}^{\Sigma}+v_{2}\right)=\left\{\exp \left(-w_{1}\right) x_{0}\right\}=\varphi\left(\mathscr{T}_{\Psi \backslash \Gamma, 0}^{\Sigma}\right) .
$$

Now let $\xi$ be tangent to $\mathfrak{T T}_{\Psi \backslash \Gamma, 0}$ at $v$. Decompose $\xi=\xi_{1}+\xi_{2}$ along $\mathfrak{M}_{\Psi \backslash \Gamma, 0}=\mathfrak{M}_{\Psi \backslash \Gamma, 0}^{\Sigma}+\Re_{\Psi \backslash \Gamma, 0}^{\Sigma}$. We have proved

$$
\left\|\varphi_{*} \xi_{1}\right\| \geqq\left\|\xi_{1}\right\|, \quad\left\|\varphi_{*} \xi_{2}\right\| \geqq\left\|\xi_{2}\right\|, \quad \varphi_{*} \xi_{1} \perp \varphi_{*} \xi_{2} .
$$

Thus

$$
\left\|\varphi_{*} \xi\right\|^{2}=\left\|\varphi_{*} \xi_{1}+\varphi_{*} \xi_{2}\right\|^{2}=\left\|\varphi_{*} \xi_{1}\right\|^{2}+\left\|\varphi_{*} \xi_{2}\right\|^{2} \geqq\left\|\xi_{1}\right\|^{2}+\left\|\xi_{2}\right\|^{2}=\|\xi\|^{2} .
$$

That completes the proof of Lemma 11.6. Q.E.D.

We need notation for the factorization of Lemma 11.6. The notation will be, for every $g \in G_{\Psi \backslash \Gamma, 0}$, that 
(11.7) $g=g_{K} \cdot g_{M} \cdot g_{R}$ where $g_{K} \in K_{\Psi \backslash \Gamma}, g_{M} \in \exp \Re_{\Psi \backslash \Gamma, 0}^{\Sigma}, g_{R} \in \exp R_{\Psi \backslash \Gamma, 0}^{\Sigma}$

11.8. Theorem. Let $\Gamma$ and $\Sigma$ be disjoint subsets of $\Psi$. Given $k \in K$, define

$\beta_{k}: k \cdot G_{\Psi \backslash \Gamma, 0}\left(x_{\Gamma, \Sigma}\right) \rightarrow k \cdot K_{\Psi \backslash \Gamma}\left(x_{\Gamma, \Sigma}\right) \quad b y \beta_{k}\left(k g x_{\Gamma, \Sigma}\right)=k g_{K}\left(x_{\Gamma, \Sigma}\right)$.

Now define

$$
\beta: G_{0}\left(x_{\Gamma, \Sigma}\right) \rightarrow K\left(x_{\Gamma, \Sigma}\right) \quad \text { by }\left.\beta\right|_{k \cdot G_{\Psi \backslash \Gamma, 0}\left(x_{\Gamma, \Sigma}\right)}=\beta_{k} .
$$

1. $\beta_{k}: k \cdot G_{\Psi \backslash \Gamma, 0}\left(x_{\Gamma, \Sigma}\right) \rightarrow k \cdot K_{\Psi \backslash \Gamma}\left(x_{\Gamma, \Sigma}\right)$ is a well-defined holomorphic bre bundle with $K_{\Psi}^{\Sigma} \backslash \bar{\Gamma}$

(1a) structure group: the connected reductive complex Lie group

(1b) total space: the homomorphic arc component $k \cdot G_{\Psi \backslash \Gamma, 0}\left(x_{\Gamma, \Sigma}\right)$; of $G_{0}\left(x_{\Sigma, \Gamma}\right)$;

(1c) base space: the maximal compact subvariety $k \cdot K_{\Psi \backslash \Gamma}\left(x_{\Gamma, \Sigma}\right)$ which is a compact totally geodesic hermitian symmetric submanifold of $X$;

(1d) fibre over $k k^{\prime}\left(x_{\Gamma, \Sigma}\right), k^{\prime} \in K_{\Psi \backslash \Gamma}$ : the totally geodesic hermitian symmetric submanifold

$$
\begin{aligned}
k k^{\prime} \cdot G_{\Psi \backslash \Gamma, 0}^{\Sigma}\left(x_{\Gamma, \Sigma}\right) & =k k^{\prime} c_{\Gamma} c_{\Sigma}^{2} \cdot G_{\Psi \backslash \Gamma, 0}^{\Sigma}\left(x_{0}\right) \\
& =k k^{\prime} c_{\Gamma} c_{\Sigma}^{2}\left\{G_{\Psi \backslash(\Gamma \cup \Sigma), 0}\left(x_{0}\right) \times G_{\Sigma, 0}^{\Sigma}\left(x_{0}\right)\right\}
\end{aligned}
$$

of noncompact type and rank $|\Psi \backslash \Gamma|$ in $k k^{\prime} c_{\Gamma} c_{\Sigma}^{2} \cdot G_{0}\left(x_{0}\right)$.

2. Let $\nu$ be the holomorphic normal bundle to $k \cdot K_{\Psi \backslash \Gamma}\left(x_{\Gamma, \Sigma}\right)$ in $k \cdot G_{\Psi \backslash \Gamma, 0}\left(x_{\Gamma, \Sigma}\right)$. Then $\nu$ is the homogeneous holomorphic vector bundle over the hermitian symmetric coset space $K_{\Psi \backslash \Gamma} / K_{\Psi \backslash \Gamma}^{\Sigma} \cong k \cdot K_{\Psi \backslash \Gamma}^{\Sigma}\left(x_{\Gamma, \Sigma}\right)$ defined by the representation of $K_{\Psi}^{\Sigma} \backslash \Gamma$ on the holomorphic tangent space of $G_{\Psi \backslash \Gamma, 0}^{\Sigma} / K_{\Psi \backslash \Gamma}^{\Sigma} \cong G_{\Psi \backslash \Gamma, 0}^{\Sigma}\left(x_{\Gamma, \Sigma}\right)$, and the bundle $\beta_{k}$ is holomorphically fibre-equivalent to a relatively compact tubular neighborhood of the zerosection of $\nu$.

3. Let det $\nu$ denote the determinant bundle of the holomorphic normal bundle $\nu$. If $\operatorname{dim}_{C} k \cdot K_{\Psi \backslash \Gamma}\left(x_{\Gamma, \Sigma}\right)>0$, then $\operatorname{det} \nu$ is a positive holomorphic line bundle (in the sense of $K$. Kodaira), and the bundles $\beta_{k}, \nu$ and $\operatorname{det} \nu$ are all topologically nontrivial. Furthermore, the following conditions are equivalent:

(3a) $\beta_{k}$ is holomorphically trivial;

(3b) $\beta_{k}$ is topologically trivial;

(3c) $\beta_{k}$ has base reduced to a point;

(3d) $G_{\Psi \backslash \Gamma, 0}\left(x_{\Gamma, \Sigma}\right)$ is hermitian symmetric; 
(3e) $\operatorname{ad}\left(c_{\Sigma}\right)$ has order 4 on $G_{\Psi \backslash \Gamma} ;$ and

(3f) If $\Delta^{m}$ are the root systems of the simple ideals of $\mathrm{G}$, then for each $m$, either $\Sigma \cap \Delta^{m}=\phi$, or $\left(\Psi \cap \Delta^{m}\right) \subset(\Gamma \cup \Sigma)$ with $G_{\Psi \cap \Delta^{m}, 0}\left(x_{0}\right)$ of tube type.

4. $\beta: G_{0}\left(x_{\Gamma, \Sigma}\right) \rightarrow K\left(x_{\Gamma, \Sigma}\right)$ is a well-defined real analytic fibre bundle whose fibres and structure group coincide with those of the restrictions $\beta_{k}$ to the holomorphic arc components $k \cdot G_{\Psi \backslash \Gamma, 0}\left(x_{\Gamma, \Sigma}\right)$ of $G_{0}\left(x_{\Gamma, \Sigma}\right)$. The bundle $\beta$ is real analytically equivalent to the homogeneous complex vector bundle over $K\left(x_{\Gamma, \Sigma}\right)$ with fibre equal to the holomorphic tangent space of $G_{\Psi}^{\Sigma} \backslash \Gamma, 0\left(x_{\Gamma, \Sigma}\right)$. The following conditions are equivalent:

(4a) $\beta$ is real analytically trivial;

(4b) $\beta$ is topologically trivial;

(4c) for each irreducible factor of $X$, the corresponding factor of $\beta$ has base or fibre reduced to a point; and

(4d) if $\Delta^{m}$ are the root systems for the simple ideals of $\mathcal{G}$, then for each $m$, either

(4di) $\Gamma \cap \Delta^{m}=\phi=\Sigma \cap \Delta^{m}$, or

(4dii) $\Gamma \cap \Delta^{m}=\Psi \cap \Delta^{m}$ and $\Sigma \cap \Delta^{m}=\phi$, or

(4diii) $\Gamma \cap \Delta^{m}=\phi$ and $\Sigma \cap \Delta^{m}=\Psi \cap \Delta^{m}$ with the bounded domain $G_{\Psi_{\cap} \Delta^{m}, 0}\left(x_{0}\right)$ of tube type.

Proof. Fix $k \in K$. Then $\beta_{k}$ is well defined by the factorization (11.7) resulting from Lemma 11.6. The total space $k \cdot G_{\Psi \backslash \Gamma, 0}\left(x_{\Gamma, \Sigma}\right)$ is a holomorphic arc component of $G_{0}\left(x_{\Gamma, \Sigma}\right)$ by Theorem 10.9, and the base space $k K_{\Psi \backslash \Gamma}\left(x_{\Gamma, \Sigma}\right) \cong K_{\Psi \backslash \Gamma} / K_{\Psi \backslash \Gamma}^{\Sigma}$ is a totally geodesic hermitian symmetric submanifold of $X$ by Lemma 11.4. That realizes $\beta_{k}$ as a real analytic fibre bundle with structure group $K_{\Psi \backslash \Gamma}^{\Sigma}$; now we may view $\left.K_{\Psi}^{\Sigma}\right\rangle_{\Gamma}^{C}$ as the structure group.

Let $k^{\prime} \in K_{\Psi \backslash \Gamma}$. The $\beta_{k}$-fibre over $k k^{\prime}\left(x_{\Gamma, \Sigma}\right)$ consists of all $k g\left(x_{\Gamma, \Sigma}\right)$ such that $k k^{\prime}\left(x_{\Gamma, \Sigma}\right)=k g_{K}\left(x_{\Gamma, \Sigma}\right)$. The latter condition is $k^{\prime}\left(x_{\Gamma, \Sigma}\right)$ $=g_{K}\left(x_{\Gamma, \Sigma}\right)$, which is $g_{K} \in k^{\prime} K_{\Psi}^{\Sigma} \backslash \Gamma$ by Lemma 11.4. Thus the fibre consists of all $k k^{\prime} k_{1} g_{M} g_{R} x_{\Gamma, \Sigma}$ with $g_{M} \in \exp \mathfrak{N}_{\Psi \backslash \Gamma, 0}^{\Sigma}$ and $g_{R} \in \exp \mathcal{R}_{\Psi}^{\Sigma} \backslash \Gamma, 0$ Lemma 11.5 says $g_{R} x_{\Gamma, \Sigma}=x_{\Gamma, \Sigma}$, and we have $k_{1} g_{M} x_{\Gamma, \Sigma}=\operatorname{ad}\left(k_{1}\right) g_{M} x_{\Gamma, \Sigma}$ which is in $\exp \left(\mathscr{N}_{\Psi}^{\Sigma} \backslash \mathrm{\Sigma}_{\mathrm{r}, 0}\right)\left(x_{\Gamma, \Sigma}\right)$. Thus the fibre consists of all $k k^{\prime} g_{M} x_{\Gamma, \Sigma}$, and now Lemma 11.3 says that it is the totally geodesic hermitian

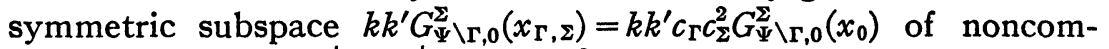
pact type and rank $|\Psi \backslash \Gamma|$ in $k k^{\prime} c_{\Gamma} c_{\Sigma}^{2} X_{0}$.

In the notation of $[15]$, it is shown $[15,85.4]$ that $\mathcal{G}^{\mathbf{Y} \backslash \Sigma}$

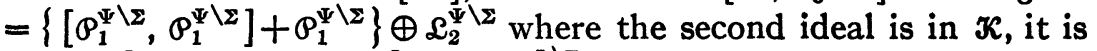

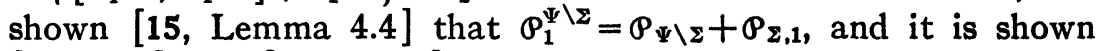
$[15,86.6]$ that $\left[\mathcal{S}_{\Psi \backslash \Sigma}, \mathcal{G}_{z, 1}\right]=0$. The 
$\mathcal{G}^{\Psi \backslash \Sigma}$ of $[15]$ is our ad $\left(\exp \frac{\pi}{4} z\right)\left\{\mathcal{G}_{u} \cap \mathcal{g}^{2}\right\}$,

$\mathcal{P}_{1}^{\Psi \backslash \Sigma}$ of [15] is our ad $\left(\exp \frac{\pi}{4} z\right)\left\{\pi_{u} \cap g^{\Sigma}\right\}$,

$\mathcal{P}_{\Psi \backslash \Sigma}$ of $[15]$ is our ad $\left(\exp \frac{\pi}{4} z\right)\left\{\Re_{u} \cap \mathcal{G}_{\Psi \backslash \Sigma}\right\}$,

$\rho_{\Sigma, 1}$ of [15] is our ad $\left(\exp \frac{\pi}{4} z\right)\left\{\Re_{u} \cap g_{\Sigma}^{\Sigma}\right\}$,

$\mathcal{S}_{\Psi \backslash \Sigma}$ of $[15]$ is our $\operatorname{ad}\left(\exp \frac{\pi}{4} z\right)\left\{\mathcal{G}_{u} \cap \mathcal{S}_{\Psi \backslash \Sigma}\right\}$,

$\oint_{\Sigma, 1}$ of $[15]$ is defined as $\left[\rho_{\Sigma, 1}, \rho_{\Sigma, 1}\right]+\rho_{\Sigma, 1}$.

Using that dictionary we conclude that

$$
\mathcal{S}_{\Psi}^{\Sigma}=\mathcal{S}_{\Psi \backslash \Sigma} \oplus \mathcal{S}_{\Sigma}^{\Sigma} \oplus \mathfrak{L}_{\Sigma}, \quad \mathfrak{L}_{\Sigma} \subset K^{C} .
$$

Taking intersection with $\mathcal{G}_{\Psi \backslash \Gamma}$, now

$$
\mathcal{S}_{\Psi \backslash \Gamma}^{\Sigma}=\mathcal{S}_{\Psi \backslash(\Gamma \cup \Sigma)} \oplus \mathcal{S}_{\Sigma}^{\Sigma} \oplus \mathfrak{L}_{\Gamma, \Sigma}, \quad \mathfrak{L}_{\Gamma, \Sigma} \subset \mathcal{K}^{c} .
$$

All three ideals are $\tau$-stable. Thus

$$
\mathcal{S}_{\Psi \backslash \Gamma, 0}^{\Sigma}=\mathcal{S}_{\Psi \backslash(\Gamma \cup \Sigma), 0} \oplus \mathcal{S}^{\Sigma} \Sigma, 0 \oplus \mathfrak{L}_{\Gamma, \Sigma, 0}, \quad \mathfrak{L}_{\Gamma, \Sigma, 0} \subset \varkappa .
$$

It follows that

$$
G_{\Psi \backslash \Gamma, 0}^{\Sigma}\left(x_{0}\right)=G_{\Psi \backslash(\Gamma \cup \Sigma), 0}\left(x_{0}\right) \times G_{\Sigma, 0}^{\Sigma}\left(x_{0}\right) .
$$

We have proved every part of (1) except for the fact that the bundle $\beta_{k}$ is holomorphic. However we have seen that its total space, base and fibres are complex submanifolds of $X$, and that its structure group is a complex Lie group acting holomorphically on the typical fibre, so we need only demonstrate the existence of holomorphic transition functions. Thus, to complete the proof of (1) it suffices to complete the proof of (2).

$G_{\Psi \backslash \Gamma, 0}\left(x_{0}\right)$ has holomorphic tangent space $\mathfrak{N}_{\Psi \backslash \Gamma}=\mathfrak{N}^{-}-\cap \mathfrak{N}_{\Psi \backslash \Gamma, 0}^{C}$ at $x_{0}$, and $\mathfrak{M}_{\Psi \backslash \Gamma}^{\Sigma}=\mathfrak{M C}^{-} \cap \mathfrak{T}_{\Psi \backslash \Gamma, 0}^{\Sigma C}$ is the subspace that is the holomorphic

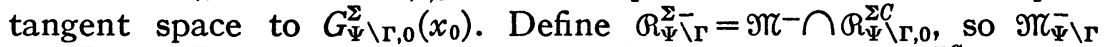
$\left.=\Re_{\Psi}^{\Sigma} \bar{\top}_{\Gamma}+\mathfrak{R}_{\Psi}^{\Sigma}\right\rangle_{\Gamma}$. Recalling that $\operatorname{ad}\left(c_{\Sigma}^{2}\right)$ interchanges $\mathfrak{R}_{\Psi}^{\Sigma C} \backslash_{\Gamma, 0}$ with $Q_{\Psi}^{\Sigma C} \backslash_{\Gamma}$ and noting that $\left.\operatorname{ad}\left(c_{\Sigma}^{4}\right) R_{\Psi}^{\Sigma}\right\rangle_{\Gamma}=R_{\Psi}^{\Sigma} \backslash_{\Gamma}$, we define $\left.Q_{\Psi}^{\Sigma} \backslash_{\Gamma}=\operatorname{ad}\left(c_{\Sigma}^{2}\right) R_{\Psi}^{\Sigma}\right\rangle_{\Gamma}$; then $\left.\operatorname{ad}\left(c_{\Sigma}^{2}\right) \pi_{\Psi} \backslash \Gamma=\operatorname{ad}\left(c_{\Sigma}^{2}\right) \pi_{\Psi}^{\Sigma}\right\rangle_{\Gamma}+Q_{\Psi}^{\Sigma} \bar{\top}_{\Gamma}$. Now Lemmas 11.3, 11.4 and 11.5 say that, at $x_{\Gamma, z}$, 
$G_{\Psi \backslash \Gamma, 0}\left(x_{\Gamma, \Sigma}\right)$ has holomorphic tangent space $\operatorname{ad}\left(c_{\Sigma}^{2}\right) \Re_{\Psi \backslash \Gamma}^{\Sigma-}+Q_{\Psi \backslash \Gamma}^{\Sigma-}$, $G_{\Psi \backslash \Gamma, 0}^{\Sigma}\left(x_{\Gamma, \Sigma}\right)$ has holomorphic tangent space $\operatorname{ad}\left(c_{\Sigma}^{2}\right) \Re_{\Psi \backslash \Gamma}^{\Sigma-}$, $K_{\Psi \backslash \Gamma}\left(x_{\Gamma, \Sigma}\right)$ has holomorphic tangent space $Q_{\Psi \backslash \Gamma}^{\Sigma-}$.

Now the holomorphic normal space of $K_{\Psi \backslash \Gamma}\left(x_{\Gamma, \Sigma}\right)$ in $G_{\Psi \backslash \Gamma, 0}\left(x_{\Gamma, \Sigma}\right)$ at $x_{\Gamma, \Sigma}$ is $\left.\operatorname{ad}\left(c_{\Sigma}^{2}\right) \Re_{\Psi}^{\Sigma}\right\rangle_{\Gamma}$, and $K_{\Psi}^{\Sigma C} \backslash_{\Gamma}$ preserves it and acts there by its representation on the holomorphic tangent space to $G_{\Psi, \Sigma}^{\Sigma}\left(x_{\Gamma, \Sigma}\right)$. That identifies the holomorphic normal bundle $\nu$ of $k \cdot K_{\Psi \backslash \Gamma}\left(x_{\Gamma, \Sigma}\right)$ in $k \cdot G_{\Psi \backslash \Gamma}\left(x_{\Gamma, \Sigma}\right)$ with the homogeneous vector bundle over $K_{\Psi \backslash \Gamma} / K_{\Psi \backslash \Gamma}^{\Sigma}$ with fibre $\left.\operatorname{ad}\left(c_{\Sigma}^{2}\right) \Re_{\Psi}^{\Sigma}\right\rangle_{\Gamma}$ that has $K_{\Psi \backslash \Gamma}^{\Sigma}$-module structure from ad ${ }_{G}$. The Harish-Chandra realization of $G_{\Psi}^{\Sigma} \backslash \Gamma, 0\left(x_{\Gamma, \Sigma}\right)$ as a bounded domain in $\left.\operatorname{ad}\left(c_{\Sigma}^{2}\right) \Re_{\Psi}^{2}\right\rangle_{\Gamma}$ identifies $\beta_{k}$ with a relatively compact open sub-bundle of $\nu$. Now (2) is proved, and that completes the proof of (1).

Let $k$ be the canonical (holomorphic) line bundle over $k \cdot K_{\Psi \backslash \Gamma}\left(x_{\Gamma, \Sigma}\right)$. It is the holomorphic line bundle whose sections are the $(d, 0)$-forms, $d=\operatorname{dim}_{C} k \cdot K_{\Psi \backslash \Gamma}\left(x_{\Gamma, \Sigma}\right)$. Thus its dual $\kappa^{*}$ has fibre $\left.\Lambda^{d} \mathcal{Q}_{\Psi}^{\Sigma}\right\rangle_{\Gamma}$ where of course $\left.d=\operatorname{dim}_{C} Q_{\Psi}^{\Sigma}\right\rangle_{\Gamma}$. Let $\delta$ denote the representation of $K_{\Psi}^{\Sigma} \backslash \Gamma$ on the fibre of $\kappa$, so its dual $\delta^{*}$ is the representation on $\left.\Lambda^{d} \Theta_{\Psi}^{\Sigma}\right\rangle_{\Gamma}$. Define $z_{\Psi \backslash \Gamma}=(i / 2) \sum_{\Psi \backslash \Gamma} h_{\psi} \in \mathcal{K}_{\Psi \backslash \Gamma}^{\Sigma}$. Then [15, Lemma 6.2] ad( $\left.z_{\Psi \backslash \Gamma}\right)$ acts on $\mathcal{R}_{\Psi \backslash \Gamma}^{\Sigma}$ as scalar multiplication by $i / 2$. As ad $\left(c_{\Sigma}^{2}\right)$ preserves $\mathcal{K}_{\Psi \backslash \Gamma}^{\Sigma}$ and interchanges $\left.R_{\Psi}^{\Sigma}\right\rangle_{\Gamma}$ with $\left.Q_{\Psi}^{\Sigma}\right\rangle_{\Gamma}$, now ad $\left(c_{\Sigma}^{2}\right)\left(2 z_{\Psi \backslash \Gamma}\right)$ is an element of $\mathcal{K}_{\Psi}^{\Sigma} \backslash \Gamma$ that acts on $Q_{\Psi}^{\Sigma} \backslash \Gamma$ under ads as scalar multiplication by $i$. Thus

$$
\underset{z_{\Psi \backslash \Gamma}}{\Sigma}=\operatorname{ad}\left(c_{\Sigma}^{2}\right)\left(2 z_{\Psi \backslash \Gamma}\right) \text { is central in } \mathcal{K}_{\Psi \backslash \Gamma}^{\Sigma}
$$

and

$\left.\operatorname{ad}\left(z_{\Psi \backslash \Gamma}^{\Sigma}\right)\right|_{Q_{\Psi \backslash \Gamma}^{\Sigma C}}$ is the almost complex structure of $k \cdot K_{\Psi \backslash \Gamma}\left(x_{\Gamma, \Sigma}\right)$.

Let $\varepsilon \subset \mathcal{K}_{\Psi \backslash \Gamma}^{\Sigma}$ denote the Lie algebra of the kernel of the action of $K_{\Psi \backslash \Gamma}$ on $K_{\Psi \backslash \Gamma}\left(x_{\Gamma, \Sigma}\right) \cong K_{\Psi \backslash \Gamma} / K_{\Psi \backslash \Gamma}^{\Sigma}$. Now $\delta^{*}$ can be described by: $\delta^{*}\left(z_{\Psi \backslash \Gamma}\right)=\mathrm{id}, \delta^{*}(\varepsilon)=0$, and $\delta^{*}$ annihilates the orthocomplement of the image of $z_{\Psi \backslash \Gamma}^{\Sigma}$ in $\mathcal{K}_{\Psi \backslash \Gamma}^{\Sigma} / \mathcal{E}$. Thus we describe $\kappa$ as the homogeneous line bundle on $K_{\Psi \backslash \Gamma} / K_{\Psi \backslash \Gamma}^{\Sigma}$ induced by the character $\delta$ on $K_{\Psi \backslash \Gamma}^{\Sigma}$ such that $\delta\left(z_{\Psi}^{\Sigma} \backslash \Gamma\right)=-\mathrm{id}, \delta(\varepsilon)=0$, and $\delta$ annihilates the orthocomplement of the image of $z_{\Psi \backslash \Gamma}^{\Sigma}$ in $\kappa_{\Psi \backslash \Gamma}^{\Sigma} / \varepsilon$.

Let $\nu$ over $k \cdot K_{\Psi \backslash \Gamma}\left(x_{\Gamma, \Sigma}\right)$ denote the holomorphic normal bundle in $k \cdot G_{\Psi \backslash \Gamma}\left(x_{\Gamma, \Sigma}\right)$. We will describe the determinant bundle det $\nu$ over $k \cdot K_{\Psi \backslash \Gamma}\left(x_{\Gamma, \Sigma}\right)$ so as to be able to compare it with the canonical bundle $\kappa$. det $\nu$ has fibre $\Lambda^{a} \operatorname{ad}\left(c_{\Sigma}^{2}\right) \Re_{\Psi \backslash \Gamma}^{\Sigma} \bar{T}_{\Gamma}$ where $a=\operatorname{dim}_{C} \mathfrak{N}_{\Psi \backslash \Gamma}^{\Sigma-}$. Let $\alpha$ denote the representation of $K_{\Psi \backslash \Gamma}^{\Sigma}$ on that fibre. We know [15, Lemma 4.4] that $\operatorname{ad}\left(z_{\Psi \backslash \Gamma}\right)$ is scalar multiplication by $i$ on $\left.\operatorname{m}_{\Psi \backslash \Gamma}^{\Sigma}\right\rangle^{2}$ thus $\operatorname{ad}\left(z_{\Psi \backslash \Gamma}^{\Sigma}\right)$ is scalar multiplication by $2 i$ on $\operatorname{ad}\left(c_{\Sigma}^{2}\right) \Re_{\Psi \backslash \Gamma}^{\Sigma-}$; we conclude that $\alpha\left(z_{\Psi}^{\Sigma} \backslash \Gamma\right)$ 
$=2 i a$. Let $\mathcal{E}^{\prime} \subset \varkappa_{\Psi \backslash \Gamma}^{\Sigma}$ denote the Lie algebra of the kernel of the action of $G_{\Psi, \Gamma, 0}^{\Sigma}$ on $G_{\Psi, \Gamma, 0}^{\Sigma}\left(x_{\Gamma, \Sigma}\right) \cong G_{\Psi \backslash \Gamma, 0}^{\Sigma} / K_{\Psi \backslash \Gamma}^{\Sigma}$. Then $\mathcal{E}^{\prime}$ is orthogonal to the subalgebra $\mathcal{G}_{0}[\Psi \backslash \Gamma]$ of $\mathcal{G}_{\Psi \backslash \Gamma, 0}^{\Sigma}$ that contains $z_{\Psi \backslash \Gamma}^{\mathbb{Z}}$. Thus we can specify: $\alpha\left(z_{\Psi}^{\Sigma} \backslash \Gamma\right)=2 i a$ and $\alpha$ annihilates the orthocomplement of $z_{\Psi}^{\Sigma} \backslash \Gamma$ in $\varkappa_{\Psi}^{\Sigma} \backslash \Gamma$. Comparing representations we now conclude that $(\operatorname{det} \nu)^{d}$ $=\kappa^{-2 a}$ as holomorphic line bundles.

Recall $d=\operatorname{dim}_{c} k \cdot K_{\Psi \backslash \Gamma}\left(x_{\Gamma, \Sigma}\right)$ and $a=\operatorname{dim}_{C} G_{\Psi \backslash \Gamma, 0}^{\Sigma}\left(x_{\Gamma, \Sigma}\right)$. If $a=0$, then the noncompact type symmetric space $G_{\Psi \backslash,, 0}^{\Sigma}\left(x_{r, \Sigma}\right)$ is reduced to a point, so its rank $|\Psi \backslash \Gamma|=0$; then $\Gamma=\Psi$ so $G_{\Psi \backslash \Gamma, 0}\left(x_{\Gamma, \Sigma}\right)=x_{\Gamma, \Sigma}$ $=K_{\Psi \backslash \Gamma}\left(x_{\Gamma, \Sigma}\right)$, whence $d=0$. In other words, if $d>0$, then $a>0$.

Suppose $d>0$. Then the canonical bundle $\kappa$ of $K_{\Psi \backslash \Gamma} / K_{\Psi \backslash \Gamma}^{\Sigma}$ is negative in the sense of K. Kodaira; as $a>0$ it follows that $\kappa^{-2 a}$ is positive; thus det $\nu$ is a positive line bundle. Let $[c(\operatorname{det} \nu)] \in H^{1,1}\left(K_{\Psi \backslash \Gamma} / K_{\Psi \backslash \Gamma}^{\Sigma} ; \mathbf{Z}\right)$ denote the Chern class of $\operatorname{det} \nu$. Now $[c(\operatorname{det} \nu)]$ is represented by a

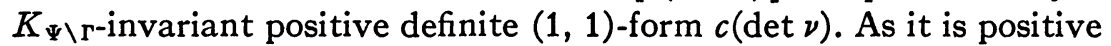

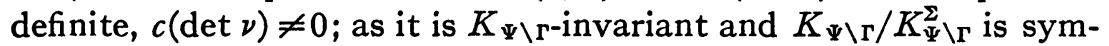
metric, now $[c(\operatorname{det} \nu)] \neq 0$; thus det $\nu$ is not topologically trivial. It follows that $\nu$ is not topologically trivial. As the bundles $\nu$ and $\beta_{k}$ are topologically equivalent, now $\beta_{k}$ is not topologically trivial.

If $d=0$, then $K_{\Psi \backslash \Gamma}=K_{\Psi \backslash \Gamma}^{\Sigma}$, so $Q_{\Psi \backslash \Gamma}^{\Sigma}=0$; then $\mathscr{Q}_{\Psi \backslash \Gamma, 0}^{\Sigma}=\operatorname{ad}\left(c_{\Sigma}^{2}\right)$ - $\left(i Q_{\Psi}^{\Sigma} \backslash \Gamma\right)=0$, so $\mathcal{S}_{\Psi \backslash \Gamma, 0}=\mathcal{S}_{\Psi \backslash \Gamma, 0}^{\Sigma}$. That says that $\operatorname{ad}\left(c_{\Sigma}\right)$ has order 4 on $\mathcal{G}_{\Psi} \backslash \Gamma$. Let $\mathcal{G}=\sum \mathcal{G}^{m}$ decomposition into simple ideals, $\Delta=U \Delta^{m}$ corresponding decomposition of the root system of $\mathrm{G}$. Then [15, Corol-

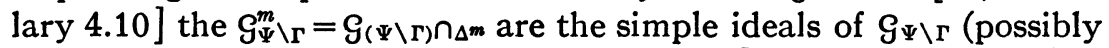
with some of them equal to zero); further [15, Corollary 4.11] if $G_{(\Psi \backslash \Gamma) \cap_{\Delta^{m}, 0}}\left(x_{0}\right)$ is of tube type and positive dimension, then $G_{\Psi \cap^{m}, 0}\left(x_{0}\right)$ is of tube type; finally [15, Theorem 5.7] ad $\left(c_{\Sigma}\right)$ has order 4 on $G_{\Psi} \backslash \Gamma$ if and only if, for each simple ideal $\mathcal{G}_{(\Psi \backslash \Gamma) \cap_{\Delta^{m}}}$ of $\mathcal{G}_{\Psi \backslash \Gamma}$, either

(i) $\Sigma \cap(\Psi \backslash \Gamma) \cap \Delta^{m}$ is empty or

(ii) $G_{(\Psi \backslash \Gamma) \cap \Delta^{m}, 0}\left(x_{0}\right)$ is of tube type and $(\Psi \backslash \Gamma) \cap \Delta^{m} \subset \Sigma$.

We conclude, using disjointness of $\Gamma$ and $\Sigma$, that $\operatorname{ad}\left(c_{\Sigma}\right)$ has order 4 on $\mathcal{S}_{\Psi \backslash \Gamma}$ if and only if, for each $m$, either

(i) $\Sigma \cap \Delta^{m}=\phi$ or

(ii) $G_{\Psi \cap \Delta^{m}, 0}\left(x_{0}\right)$ is of tube type and $\Psi \cap \Delta^{m} \subset(\Gamma \cup \Sigma)$.

That completes the proof of (3).

We will prove $\beta: G_{0}\left(x_{\Gamma, \Sigma}\right) \rightarrow K\left(x_{\Gamma, \Sigma}\right)$ well-defined. In other words, given $k, k^{\prime} \in K$ with $k \cdot G_{\Psi \backslash \Gamma, 0}\left(x_{\Gamma, \Sigma}\right)=k^{\prime} \cdot G_{\Psi \backslash \Gamma, 0}\left(x_{\Gamma, \Sigma}\right)$, we will prove $\beta_{k}=\beta_{k^{\prime}}$. Now suppose

$$
x \in k \cdot G_{\Psi \backslash \Gamma, 0}\left(x_{\Gamma, \Sigma}\right)=k^{\prime} \cdot G_{\Psi \backslash \Gamma, 0}\left(x_{\Gamma, \Sigma}\right) ; \quad k, k^{\prime} \in K .
$$

Express

$$
x=k g\left(x_{\Gamma, \Sigma}\right)=k^{\prime} g^{\prime}\left(x_{\Gamma, \Sigma}\right) ; \quad g, g^{\prime} \in G_{\Psi \backslash \Gamma, 0}
$$


$k^{-1} k^{\prime} \in K$ preserves the holomorphic arc component $G_{\Psi \backslash \Gamma, 0}\left(x_{\Gamma, \Sigma}\right)$ of $G_{0}\left(x_{\Gamma, \Sigma}\right)$; that holomorphic arc component being a symmetric space of noncompact type, now $k^{-1} k^{\prime}=k_{1} k_{2}$ where $k_{1} \in K_{\Psi \backslash \Gamma}$ and $k_{2}$ acts trivially on $G_{\Psi \backslash \Gamma, 0}\left(x_{\Gamma, \Sigma}\right)$. Thus we may ignore $k_{2}$ and assume

$$
k^{\prime}=k k_{1} \quad \text { with } k_{1} \in K_{\Psi \backslash \Gamma} .
$$

As in (11.7) we decompose

$$
g=g_{K} g_{M} g_{R} \text { and } g^{\prime}=g_{K}^{\prime} g_{M}^{\prime} g_{R}^{\prime}
$$

Using $g_{R} x_{\Gamma, \Sigma}=x_{\Gamma, \Sigma}=g_{R}^{\prime} x_{\Gamma, \Sigma}$, now we have

$$
x=k g_{K} g_{M} x_{\Gamma, \Sigma}=k k_{1} g_{K}^{\prime} g_{M}^{\prime} x_{\Gamma, \Sigma} .
$$

Thus $g_{K} g_{M} h=k_{1} g_{K}^{\prime} g_{M}^{\prime}$ where $h \in G_{\Psi \backslash \Gamma, 0}$ fixes $x_{\Gamma, \Sigma}$. Lemma 11.5 says $h=h_{R} h_{R}, h_{K} \in K_{\Psi \backslash \Gamma}^{\Sigma}$ and $h_{R} \in \exp \Re_{\Psi}^{\Sigma} \backslash \Gamma, 0$. Now

$$
\left(k_{1} g_{K}^{\prime}\right) g_{M}^{\prime}=g_{K} g_{M} h_{K} h_{R}=\left(g_{K} h_{K}\right)\left(h_{K}^{-1} g_{M} h_{K}\right) h_{R}
$$

Uniqueness of (11.7) thus says

$$
k_{1} g_{K}^{\prime}=g_{K} h_{K}, \quad g_{M}^{\prime}=h_{K}^{-1} g_{M} h_{K}, \quad 1=h_{R}
$$

Thus

$$
\beta_{k}(x)=k g_{K}\left(x_{\Gamma, \Sigma}\right)=k g_{K} h_{K}\left(x_{\Gamma, \Sigma}\right)=k k_{1} g_{K}^{\prime}\left(x_{\Gamma, \Sigma}\right)=k^{\prime} g_{K}^{\prime}\left(x_{\Gamma, \Sigma}\right)=\beta_{k^{\prime}}(x),
$$

and $\beta$ is proved to be well defined.

Now $\beta: G_{0}\left(x_{\Gamma, \Sigma}\right) \rightarrow K\left(x_{\Gamma, \Sigma}\right)$ is a well-defined real analytic map. If $x \in G_{0}\left(x_{\Gamma, \Sigma}\right)$, then $x$ and $\beta(x)$ are in the same holomorphic arc component of $G_{0}\left(x_{\Gamma, \Sigma}\right)$. It follows that $\beta$ is a real analytic bundle whose fibres coincide with those of the restrictions $\beta_{k}$. The structure group of $\beta$ is the isotropy subgroup of $K$ at $x_{\Gamma, \Sigma}$. That isotropy group preserves the holomorphic arc component $G_{\Psi \backslash \Gamma, 0}\left(x_{\Gamma, \Sigma}\right)$, so its every element has factorization $k=k_{1} k_{2}$ with $k_{1} \in G_{\Psi \backslash \Gamma, 0} \cap K=K_{\Psi \backslash \Gamma}$ and $k_{2}$ acting trivially on the fibre $G_{\Psi \backslash \Gamma, 0}\left(x_{\Gamma, \Sigma}\right)$ of $\beta$. Note $k_{1} \in K_{\Psi \backslash \Gamma}^{\Sigma}$ because $k_{1}\left(x_{\Gamma, \Sigma}\right)=x_{\Gamma, \Sigma}$. Thus we can reduce the structure group of $\beta$ to the structure group $K_{\Psi \backslash \Gamma}^{\Sigma}$ of its restrictions $\beta_{k}$.

Consider the special case $\beta: G_{0}\left(x_{\Gamma, \phi}\right) \rightarrow K\left(x_{\Gamma, \phi}\right)$ where $G_{0}$ is simple and $\phi \neq \Gamma \neq \Psi$. The fibre of $\beta$ over $k\left(x_{\Gamma, \phi}\right)$ is the holomorphic arc component $k \cdot G_{\Psi \backslash \Gamma, 0}\left(x_{\Gamma, \phi}\right)$ of $G_{0}\left(x_{\Gamma, \phi}\right)$. The space $K\left(x_{\phi, \Gamma}\right)$, maximal compact subvariety of the open orbit $G_{0}\left(x_{\phi, \Gamma}\right)$, has been seen to be a totally geodesic hermitian symmetric subspace of compact type in $X$. We will construct a positive holomorphic line bundle $\lambda$ over $K\left(x_{\phi, \Gamma}\right)$, and a fibration $f: K\left(x_{\Gamma, \phi}\right) \rightarrow K\left(x_{\phi, \Gamma}\right)$, such that 
$f^{*} \lambda$ is the determinant bundle of $\beta: G_{0}\left(x_{\Gamma, \phi}\right) \rightarrow K\left(x_{\Gamma, \phi}\right)$.

For that construction we use the notation

$$
\begin{aligned}
& L^{\Gamma} \text { : isotropy subgroup of } K \text { at } x_{\Gamma, \phi} ; \\
& K^{\Gamma} \text { : isotropy subgroup of } K \text { at } x_{\phi, \Gamma} .
\end{aligned}
$$

Lemma 11.4 says that $K^{\mathrm{r}}=K_{\Psi}^{\mathrm{r}}$, centralizer of $c_{\Gamma}^{4}$ in $K$; the decomposition $\mathcal{S}_{\Psi, \phi}^{\Gamma}=\mathfrak{L}_{\Gamma, \phi, 0} \oplus \mathcal{G}_{\Psi \backslash \Gamma, 0}+\mathcal{G}_{\Gamma, 0}^{\Gamma}$ informs us first that $L^{\Gamma}=L_{\Gamma, \phi, 0} \cdot K_{\Psi \backslash \Gamma}$ local direct product where the first factor acts trivially on $G_{\Psi \backslash \Gamma, 0}\left(x_{\Gamma, \phi}\right)$, and second that $K^{\mathrm{r}}=L^{\mathrm{r}} \cdot K_{\Gamma}^{\mathrm{r}}$ local direct product. Now we define

$$
f: K\left(x_{\Gamma, \phi}\right)=K / L^{\Gamma} \rightarrow K / K^{\Gamma}=K\left(x_{\phi, \Gamma}\right) \quad \text { by } f\left(k L^{\Gamma}\right)=k K^{\Gamma} .
$$

View $f$ as identification under the right action of $K_{\Gamma}^{\Gamma}$ on $K / L^{\Gamma}$. That action commutes with the action of $L^{\Gamma}$ on the fibres of $\beta$. Thus, in the category of topological vector bundles,

$$
\beta=f^{*} \gamma \text { for some vector bundle } \gamma \text { over } K\left(x_{\phi, \mathrm{r}}\right) \text {. }
$$

As $K^{\Gamma}$ acts on the fibres $k \cdot G_{\Psi \backslash \Gamma, 0}\left(x_{\Gamma, \phi}\right)$ of $\gamma$ via the action of $K_{\Psi \backslash \Gamma}^{C}$ on $G_{\Psi \backslash \Gamma, 0}\left(x_{\Gamma, \phi}\right)$, which is holomorphic,

$\gamma$ over $K\left(x_{\phi, \Gamma}\right)$ is the $K$-homogeneous holomorphic

vector bundle with fibre $\operatorname{TI}_{\bar{\Psi} \backslash \Gamma}^{-}$over $x_{\phi, \Gamma}$.

Now we compare det $\boldsymbol{\gamma}$ with the canonical bundle $\kappa$ over $K\left(x_{\phi, \Gamma}\right)$. $\operatorname{ad}\left(c_{\Gamma}^{2}\right) z$ acts on the fibre $\Lambda^{a} \mathfrak{N}_{\bar{\Psi} \backslash_{\Gamma}}, a=\operatorname{dim}_{C} \mathfrak{M}_{\bar{\Psi} \backslash \Gamma}$, of det $\gamma$, as multiplication by $i a$. It acts on the fibre $\left(\Lambda^{d} Q_{\Psi}^{\Gamma^{-}}\right)^{*}=\left(\Lambda^{d} \operatorname{ad}\left(c_{\Gamma}^{2}\right) R_{\Psi}^{\Gamma^{-}}\right)^{*}$, $d=\operatorname{dim}_{C} Q_{\Psi}^{\Gamma-}=\operatorname{dim}_{C} K\left(x_{\phi}, \Gamma\right)$, of $\kappa$, as multiplication by $-\mathrm{id}$. Thus as before,

$$
(\operatorname{det} \gamma)^{d}=\kappa^{-a} \text {. }
$$

Our hypothesis that $G_{0}$ be simple and $\phi \neq \Gamma \neq \Psi$ ensures $d \neq 0 \neq a$. As $\kappa$ is negative, now $(\operatorname{det} \gamma)^{d}$ is positive, so $\operatorname{det} \gamma$ is positive. Define $\lambda=\operatorname{det} \gamma$, and we have

$$
f^{*} \lambda=f^{*} \operatorname{det} \gamma=\operatorname{det} f^{*} \gamma=\operatorname{det}\left(\beta: G_{\Psi \backslash \Gamma, 0}\left(x_{\Gamma, \phi}\right) \rightarrow K\left(x_{\Gamma, \phi}\right)\right) .
$$

That completes the construction.

Suppose $G_{0}$ simple. We will prove that $\beta: G_{0}\left(x_{r, \Sigma}\right) \rightarrow K\left(x_{r, z}\right)$ is topologically trivial if and only if

(i) $\Gamma=\phi=\Sigma$, so $\beta$ is fibration of $G_{0}\left(x_{0}\right)=X_{0}$ over the single point $x_{0}$, or

(ii) $\Gamma=\Psi$ and $\Sigma=\phi$, so $\beta: G_{0}\left(x_{\Psi, \phi}\right) \rightarrow K\left(x_{\Psi, \phi}\right)$ is the fibration of the Bergman-Silov boundary of $X_{0}$ in $X$ over itself with fibre reduced to a point; or 
(iii) $\Gamma=\phi, \Sigma=\Psi$, and $X_{0}=G_{0}\left(x_{0}\right)$ is of tube type, so $\beta$ is fibration of $G_{0}\left(x_{\phi, \Psi}\right)=c_{\Psi}^{2} X_{0}$ over a single point $x_{\phi, \Psi}=c_{\Psi}^{2} x_{0}$.

First note that $\beta$ is topologically trivial in the three cases described just above. Conversely, now assume $\beta$ topologically trivial. Then the restrictions $\beta_{k}$ to the holomorphic arc components $k \cdot G_{\Psi \backslash \Gamma, 0}\left(x_{\Gamma, \Sigma}\right)$ of $G_{0}\left(x_{\Gamma, \Sigma}\right)$ are topologically trivial, so either $\Sigma=\phi$, or $\Psi=\Gamma \cup \Sigma$ with $X_{0}$ of tube type. First suppose $\Sigma=\phi$, so $\beta: G_{0}\left(x_{\Gamma, \phi}\right) \rightarrow K\left(x_{\Gamma, \phi}\right)$, and recall the map $f: K\left(x_{\Gamma, \phi}\right) \rightarrow K\left(x_{\phi, \Gamma}\right)$ of the paragraph before last. If $\phi \neq \Gamma \neq \Psi$, we saw that the determinant bundle of $\beta$, viewing $\beta$ topologically as a complex vector bundle, is of the form $f^{*} \lambda$ where $\lambda$ over $K\left(x_{\phi, \Gamma}\right)$ is a positive holomorphic line bundle. As its Chern class is nonzero, $\lambda$ is not topologically trivial, so $f^{*} \lambda=\operatorname{det} \beta$ is not topologically trivial, contradicting topological triviality of $\beta$. Thus either $\Gamma=\phi$ and we are in case (i) above, or $\Gamma=\Psi$ and we are in case (ii) above. Now suppose $\Sigma \neq \phi$, so $\Psi=\Gamma \cup \Sigma$ with $X_{0}$ of tube type. As $X_{0}$ is of tube type, i.e. as $c_{\Psi}^{4}=1$, we have $K$ and $G_{0}$ stable under $\operatorname{ad}\left(c_{\Psi}^{2}\right)$. Now, using the consequence $c_{\Psi}=c_{\Gamma} c_{\Sigma}$ of $\Psi=\Gamma \cup \Sigma$ and $\Gamma \cap \Sigma=\phi$, we have

$$
c_{\Psi}^{2}: G_{0}\left(x_{\Gamma, \Sigma}\right) \rightarrow G_{0}\left(c_{\Psi}^{2} x_{\Gamma, \Sigma}\right)=G_{0}\left(c_{\Gamma}^{3} c_{\Sigma}^{4} x_{0}\right)=G_{0}\left(x_{\Gamma, \phi}\right),
$$

and that the map has the properties

$$
\stackrel{2}{c}_{c_{\Psi}} K\left(x_{\Gamma, \Sigma}\right)=K\left(x_{\Gamma, \phi}\right) \quad \text { and } \quad \stackrel{2}{c_{\Psi}} k \cdot G_{\Psi \backslash \Gamma, 0}\left(x_{\Gamma, \Sigma}\right)=k^{\prime} \cdot G_{\Psi \backslash \Gamma, 0}\left(x_{\Gamma, \phi}\right) .
$$

Thus $c_{\Psi}^{2}$ defines a fibre bundle isomorphism between the fibrations $\beta$ given by

$$
G_{0}\left(x_{\Gamma, \Sigma}\right) \rightarrow K\left(x_{\Gamma, \Sigma}\right) \text { and } G_{0}\left(x_{\Gamma, \phi}\right) \rightarrow K\left(x_{\Gamma, \phi}\right) .
$$

The first being topologically trivial by hypothesis, the second must be trivial, and we have just seen that to imply either $\Gamma=\phi$ or $\Gamma=\Psi$. But $\Psi=\Gamma \cup \Sigma, \Gamma \cap \Sigma=\phi$ and $\Sigma \neq \phi \phi$; thus $\Gamma=\phi, \Sigma=\Psi$ and we are in case (iii) above.

Let $\mathcal{G}^{m}$ denote the simple ideals of $\mathcal{G}, \Delta^{m}$ the root system of $\mathcal{G}^{m}$, $\mathcal{G}_{0}^{m}=\mathcal{G}_{0} \cap \mathcal{G}^{m}, G^{m}$ and $G_{0}^{m}$ the analytic subgroups for $\mathcal{G}^{m}$ and $\mathcal{G}_{0}^{m}$, and $X^{m}=G^{m}\left(x_{0}\right)$ and $X_{0}^{m}=G_{0}^{m}\left(x_{0}\right)$. Then $G_{0}\left(x_{\Gamma, \Sigma}\right)$ is the product of the $G_{0}^{m}\left(x_{\Gamma \cap \Delta^{m}, \Sigma \cap \Delta^{m}}\right)$, and the base and fibres of $\beta: G_{0}\left(x_{\Gamma, \Sigma}\right) \rightarrow K\left(x_{\Gamma, \Sigma}\right)$ decompose similarly. Thus $\beta$ is topologically trivial if and only if each of the "factors"

$$
G_{0}^{n}\left(x_{\Gamma} \cap \Delta^{m}, \Sigma \cap \Delta^{m}\right) \rightarrow\left(K \cap G_{0}^{m}\right)\left(x_{\Gamma} \cap \Delta^{m}, \Sigma \cap \Delta^{m}\right)
$$

is topologically trivial. According to the paragraph before the last one, $\beta$ is topologically trivial if and only if, for each $m$,

(i) $\Psi \cap \Delta^{m}=\phi=\Sigma \cap \Delta^{m}$, or 
(ii) $\Gamma \cap \Delta^{m}=\Psi \cap \Delta^{m}$ and $\Sigma \cap \Delta^{m}=\phi$, or

(iii) $\Gamma \cap \Delta^{m}=\phi$ and $\Sigma \cap \Delta^{m}=\Psi \cap \Delta^{m}$ with $X_{0}^{m}$ of tube type.

That completes the proof of (4), completing the proof of Theorem 11.8. Q.E.D.

11.9. Corollary. Let $\Gamma \subset \Psi$. Then the fibration $\beta: G_{0}\left(x_{\Gamma, \phi}\right) \rightarrow K\left(x_{\Gamma, \phi}\right)$ of Theorem 11.8 is given by $\beta\left(k \cdot G_{\Psi \backslash \Gamma, 0}\left(x_{\Gamma, \phi}\right)\right)=k\left(x_{\Gamma, \phi}\right)$ for $k \in K$, and it is real analytically equivalent to a complex homogeneous vector bundle $\mu$ over $K\left(x_{\Gamma, \phi}\right)$. There is a fibration $f: K\left(x_{\Gamma, \phi}\right) \rightarrow K\left(x_{\phi, \Gamma}\right)$ defined by $f\left(k x_{\Gamma, \phi}\right)=k x_{\phi, \Gamma}$, whose fibres are compact totally geodesic submanifolds $k \cdot K_{\Psi}^{\Gamma}\left(x_{\Gamma, \phi}\right)$ of $X$, and whose base is the totally geodesic hermitian symmetric subspace $K\left(x_{\phi, \Gamma}\right)$ of $X ; \mu=f^{*} \gamma$ for a certain holomorphic homogeneous vector bundle $\gamma$ over $K\left(x_{\phi, \Gamma}\right)$. If $\phi \neq \Gamma \cap \Delta^{m} \neq \Psi \cap \Delta^{m}$ where $\Delta^{m}$ is the root system of some simple ideal of $\mathcal{G}$, then $\operatorname{dim}_{C} K\left(x_{\phi, \Gamma}\right)>0$ and det $\boldsymbol{\gamma}$ is a positive homogeneous holomorphic line bundle over $K\left(x_{\phi, \Gamma}\right)$; in particular, in that case $\operatorname{det} \gamma, \gamma$, det $\mu, \mu$ and $\beta$ all are topologically nontrivial.

11.10. Corollary. Let $\Gamma \subset \Psi$ and $\Sigma=\Psi \backslash \Gamma$ with $X_{0}$ of tube type. Then the fibration $\beta: G_{0}\left(x_{\Gamma, \Sigma}\right) \rightarrow K\left(x_{\Gamma, \Sigma}\right)$ of Theorem 11.8 is given by mapping $c_{\Psi}^{2} k G_{\Psi \backslash \Gamma, 0}\left(x_{\Gamma, \phi}\right)=\operatorname{ad}\left(c_{\Psi}^{2}\right) k \cdot G_{\Psi \backslash \Gamma, 0}\left(x_{\Gamma, \Sigma}\right)$ to $c_{\Psi}^{2} k x_{\Gamma, \phi}=\operatorname{ad}\left(c_{\Psi}^{2}\right) k \cdot x_{\Gamma, \Sigma}$, and it is real analytically equivalent to a complex homogeneous vector bundle $\mu^{\prime}$ over $K\left(x_{\Gamma, \Sigma}\right)$. There is a fibration $f^{\prime}: K\left(x_{\Gamma, \Sigma}\right) \rightarrow K\left(x_{\phi, \Sigma}\right)$ defined by $f^{\prime}\left(k x_{\Gamma, \Sigma}\right)=k x_{\phi, \Sigma}$, whose fibres are compact totally geodesic submanifolds $k \cdot K_{\Psi}^{\mathbf{\Gamma}}\left(x_{\Gamma, \Sigma}\right)=k \cdot K_{\Psi}^{\Sigma}\left(x_{\Gamma, \Sigma}\right)$ of $X$, and whose base is the totally geodesic hermitian symmetric subspace $K\left(x_{\phi, \Sigma}\right)$ of $X ; \mu^{\prime}=f^{\prime *} \gamma^{\prime}$ where $\gamma^{\prime}$ is a certain holomorphic homogeneous vector bundle over $K\left(x_{\phi, \Sigma}\right)$. If $\phi \neq \Sigma$ $\cap \Delta^{m} \neq \Psi \cap \Delta^{m}$ where $\Delta^{m}$ is the root system of some simple ideal of $\mathcal{G}$, then $\operatorname{dim}_{C} K\left(x_{\phi}, \Sigma\right)>0$ and $\operatorname{det} \gamma^{\prime}$ is a positive homogeneous holomorphic line bundle over $K\left(x_{\phi, \Sigma}\right)$; in particular, in that case $\operatorname{det} \gamma^{\prime}, \gamma^{\prime}, \operatorname{det} \mu_{.}^{\prime}, \mu^{\prime}$ and $\beta$ are all topologically nontrivial.

Corollaries 10.9 and 10.10 are related by: $c_{\Psi}^{2}$ is a bundle isomorphism of $G_{0}\left(x_{\Gamma, \Sigma}\right) \rightarrow K\left(x_{\Gamma, \Sigma}\right)$ onto $G_{0}\left(x_{\Gamma, \phi}\right) \rightarrow K\left(x_{\Gamma, \phi}\right)$ and maps $K\left(x_{\phi, \Sigma}\right)$ onto $K\left(x_{\phi, \Gamma}\right)$, inducing the fibrations $f^{\prime}$ from $f, \mu^{\prime}$ from $\mu$ and $\gamma^{\prime}$ from $\gamma$.

Proof of Corollaries. Corollary 11.9 is contained in the proof of part 4 of Theorem 11.8 for the case where $G$ is simple, and the general case follows. The second paragraph of the statement of Corollary $\mathbf{1 1 . 1 0}$ is also contained in that proof, and the first paragraph then follows from Corollary 11.9. Q.E.D.

The special case of an open orbit is worth separate examination. 
11.11. CoROllaRY. Every open $G_{0}$-orbit on $X$ has a holomorphic fibration

$$
\beta: G_{0}\left(x_{\phi, \Sigma}\right) \rightarrow K\left(x_{\phi, \Sigma}\right) \quad \text { by } \quad \beta\left(g x_{\phi, \Sigma}\right)=g_{K} x_{\phi, \Sigma}
$$

where

$$
g=g_{K} g_{M} g_{R}, \quad g_{K} \in K, \quad g_{M} \in \exp \Re_{\Psi, 0}^{\Sigma}, \quad g_{R} \in \exp R_{\Psi, 0}^{\Sigma} .
$$

1. The bundle $\beta$ has structure group $K_{\Psi}^{\Sigma}$, has total space $G_{0}\left(x_{\phi, \Sigma}\right)$, has base space $K\left(x_{\phi, \Sigma}\right)$ which is a compact totally geodesic hermitian symmetric submanifold of $X$, and has fibre over $k\left(x_{\phi, \Sigma}\right)$ which is the totally geodesic hermitian symmetric submanifold

$$
k \cdot G_{\Psi, 0}^{\Sigma}\left(x_{\phi, \Sigma}\right)=k c_{\Sigma}^{2} G_{\Psi, 0}^{\Sigma}\left(x_{0}\right)=k c_{\Sigma}^{2}\left\{G_{\Psi \backslash \Sigma, 0}\left(x_{0}\right) \times G_{\Sigma, 0}^{\Sigma}\left(x_{0}\right)\right\}
$$

of noncompact type and rank $|\Psi|$ in $k c_{2}^{2} G_{0}\left(x_{0}\right)$.

2. The holomorphic normal $\nu$ bundle of $K\left(x_{\phi, \Sigma}\right)$ in $G_{0}\left(x_{\phi, \Sigma}\right)$ is the homogeneous vector bundle over $K\left(x_{\phi}, \Sigma\right) \cong K / K_{\Psi}^{\Sigma}$ associated to the representation of $K_{\Psi}^{\Sigma}$ on the holomorphic tangent space of $G_{\Psi, 0}^{\Sigma} / K_{\Psi}^{\Sigma}$. The bundle $\beta$ is holomorphically equivalent to a relatively compact open subbundle of that holomorphic normal bundle $\nu$. If $\operatorname{dim}_{C} K\left(x_{\phi, \Sigma}\right)>0$, i.e. if the root system $\Delta^{m}$ of some simple ideal of $\mathcal{G}$ satisfies $\Sigma \cap \Delta^{m} \neq \phi$, and satisfies $\Sigma \cap \Delta^{m} \neq \Psi \cap \Delta^{m}$ in case $G_{\Psi \cap \Delta m, 0}\left(x_{0}\right)$ is of tube type, then $\operatorname{det} \nu$ is a positive homogeneous holomorphic line bundle over $K\left(x_{\phi, \Sigma}\right)$, and the bundles $\operatorname{det} \nu, \nu$, and $\beta$ all are topologically nontrivial.

PRoof. This is the case $\Gamma=\phi$ of Theorem 11.8. Q.E.D.

The case of the "opposite" open orbit is of further interest because it gives new characterizations of tube domains.

11.12. Corollary. The "opposite" open orbit $G_{0}\left(x_{\phi, \Psi}\right) \subset X$ has a holomorphic fibration

$$
\beta: G_{0}\left(x_{\phi, \Psi}\right) \rightarrow K\left(x_{\phi, \Psi}\right) \quad \text { by } \quad \beta\left(g x_{\phi, \Psi}\right)=g_{K} x_{\phi, \Psi}
$$

where

$$
g=g_{K} g_{M} g_{R}, \quad g_{K} \in K, \quad g_{M} \in \exp \mathfrak{M}_{\Psi, 0}^{\Psi}, \quad g_{R} \in \exp \mathcal{R}_{\Psi, 0}^{\Psi} .
$$

1. The bundle $\beta$ has structure group $K_{\Psi}^{\Psi C}$, total space $G_{0}\left(x_{\phi, \Psi}\right)$, base space $K\left(x_{\phi, \Psi}\right)$ which is a maximal compact subvariety of $G_{0}\left(x_{\phi, \Psi}\right)$ and is a compact totally geodesic hermitian symmetric submanifold of $X$, and has fibre over $k\left(x_{\phi, \Psi}\right)$ which is the "tube part" (c.f. [2])

$$
k \cdot G_{\Psi, 0}^{\Psi}\left(x_{\phi, \Psi}\right)=k c_{\Psi}^{2} \cdot G_{\Psi, 0}^{\Psi}\left(x_{0}\right)
$$

of $k c_{\Psi}^{2} \cdot G_{0}\left(x_{0}\right)$. 
2. The bundle $\beta$ is a relatively compact open subbundle of the holomorphic normal bundle $\nu$ of $K\left(x_{\phi, \Psi}\right)$ in $G_{0}\left(x_{\phi, \Psi}\right), \nu$ is the homogeneous holomorphic vector bundle over $K\left(x_{\phi, \Psi}\right)=K / K_{\Psi}^{\Psi}$ associated to the representation of $K_{\Psi}^{\Psi}$ on the holomorphic tangent space of the tube part $G_{\Psi, 0}^{\Psi}\left(x_{0}\right)$ of $G_{0}\left(x_{0}\right)$.

3. The following conditions are equivalent:

(3a) $\beta$ is holomorphically trivial.

(3b) $\nu$ is holomorphically trivial.

(3c) $\operatorname{det} \nu$ is holomorphically trivial.

(3d) $\beta$ is topologically trivial.

(3e) $\nu$ is topologically trivial.

(3f) $\operatorname{det} \nu$ is topologically trivial.

(3g) $K\left(x_{\phi, \Psi}\right)$ is the single point $x_{\phi, \Psi}$.

(3h) $c_{\Psi}$ has order 4 on $X$.

(3i) The bounded symmetric domain $X_{0}=G_{0}\left(x_{0}\right)$ is of tube type, i.e. it has a holomorphic realization as a tube domain over a homogeneous self dual cone.

(3j) The Bergman-Šilov boundary $G_{0}\left(x_{\Psi, \phi}\right)$ of $X_{0}$ in $X$ is a totally geodesic submanifold of $X$.

(3k) $\operatorname{dim}_{\mathbb{R}} G_{0}\left(x_{\Psi, \phi}\right)=\operatorname{dim}_{C} X$.

(31) Viewing $G_{0}$ as the identity component of the group $G_{R}$ of real points in the linear algebraic group $G$, we have $P_{x \Psi, \phi}$ defined $/ R$, i.e. $X$ is defined $/ \mathrm{R}$.

Proof. Statements 1 and 2, and equivalence of (3a) through (3h), are the case $\Sigma=\Psi$ of Corollary 11.11. Equivalence of (3h), (3i), (3j) and ( $3 \mathrm{k})$ is contained in [2, Theorems 4.9 and 6.8], and Theorem 3.6 says that (3k) is equivalent to (31). Q.E.D.

We recapitulate the decomposition of an arbitrary $G_{0}$-orbit $G_{0}\left(x_{\Gamma, \Sigma}\right) \subset X$. Here $\Gamma$ and $\Sigma$ are disjoint subsets of $\Psi$. First we have the fibration of Theorem 11.8,

$$
\beta: G_{0}\left(x_{\Gamma, \Sigma}\right) \rightarrow K\left(x_{\Gamma, \Sigma}\right),
$$

that maps an arbitrary holomorphic arc component $k \cdot G_{\Psi \backslash \Gamma, 0}\left(x_{\Gamma, \Sigma}\right)$ holomorphically onto its maximal compact subvariety $k \cdot K_{\Psi \backslash \Gamma}\left(x_{\Gamma, \Sigma}\right)$; the fibre over $k k^{\prime}\left(x_{\Gamma, \Sigma}\right), k \in K$ and $k^{\prime} \in K_{\Psi \backslash \Gamma}$, is

$$
k k^{\prime} \cdot G_{\Psi \backslash \Gamma, 0}^{\Sigma}\left(x_{\Gamma, \Sigma}\right)=k k^{\prime} c_{\Gamma} c_{\Sigma}^{2}\left\{G_{\Psi \backslash(\Gamma \cup \Sigma), 0}\left(x_{0}\right) \times G_{\Sigma, 0}^{\Sigma}\left(x_{0}\right)\right\} .
$$

We then follow $\beta$ by $\alpha \cdot \beta^{-1}$ where

$$
\alpha: G_{0}\left(x_{\Gamma, \Sigma}\right) \rightarrow G_{0} / N_{r, \Sigma, 0}
$$


is the fibration of the orbit over the space of its holomorphic arc components. This amounts to collapsing each of the maximal compact subvarieties $k \cdot K_{\Psi \backslash \Gamma}\left(x_{\Gamma, \Sigma}\right) \subset k \cdot G_{\Psi \backslash \Gamma, 0}\left(x_{\Gamma, \Sigma}\right)$ to a point. That collapsing is, of course, already done when, whenever $\Delta^{m}$ is the root system of a simple ideal of $\mathcal{G}$, either $\Sigma \cap \Delta^{m}=\phi$, or $(\Gamma \cup \Sigma) \cap \Delta^{m}=\Psi \cap \Delta^{m}$ with $G_{\Psi} \cap_{\Delta^{m}, 0}\left(x_{0}\right)$ of tube type. Now we come to the final stage of the decomposition, which is realization of the $\beta$-fibre

$$
G_{\Psi \backslash \Gamma, 0}^{\Sigma}\left(x_{\Gamma, \Sigma}\right)=G_{\Psi \backslash(\Gamma \cup \Sigma), 0}\left(x_{\Gamma, \Sigma}\right) \times G_{\Sigma, 0}^{\Sigma}\left(x_{\Gamma, \Sigma}\right)
$$

as a Siegel domain of type III over any of its boundary components. That Siegel domain realization is the content of Wolf-Koranyi [15, $\S 7]$; the appendix to this $\$ 11$ transcribes it into our notation, and explicitly works out the holomorphic fibration over the boundary component (Proposition 11 A.3) that is implicit in the Siegel domain formulation, for the hermitian symmetric space $X_{0}=G_{0}\left(x_{0}\right)$. We now recast the results of that appendix to this $\$ 11$ to cover our case of the hermitian symmetric space

$$
\begin{aligned}
G_{\Psi \backslash \Gamma, 0}^{\Sigma}\left(x_{\Gamma, \Sigma}\right) & =G_{\Psi \backslash(\Gamma \cup \Sigma), 0}\left(x_{\Gamma, \Sigma}\right) \times G_{\Sigma, 0}^{\Sigma}\left(x_{\Gamma, \Sigma}\right) \\
& =c_{\Gamma} c_{\Sigma}^{2}\left\{G_{\Psi \backslash(\Gamma \cup \Sigma), 0}\left(x_{0}\right) \times G_{\Sigma, 0}^{\Sigma}\left(x_{0}\right)\right\}=c_{\Gamma} c_{\Sigma}^{2} G_{\Psi \backslash \Gamma, 0}^{\Sigma}\left(x_{0}\right) .
\end{aligned}
$$

$\Psi \backslash \Gamma$ is the maximal set of strongly orthogonal noncompact positive roots of $\mathcal{S}_{\Psi}^{\Sigma} \backslash \Gamma, 0$. Let $E$ be any subset. Then we have disjoint unions $\Psi \backslash(\Gamma \cup \Sigma)=A \cup A^{\prime}, \quad \Sigma=B \cup B^{\prime}, \quad E=A \cup B, \quad E^{\prime}=A^{\prime} \cup B^{\prime}$.

Now observe

$$
\begin{aligned}
& \mathfrak{M}_{\Psi \backslash \Gamma}^{\Sigma-} \cap \mathfrak{M}_{E}^{E-}=\mathfrak{M}_{A}^{A-}+\mathfrak{M}_{B}^{B-}, \\
& \mathfrak{N}_{\Psi \backslash \Gamma}^{E-} \cap{R_{\Psi}^{E-}}_{\Psi}^{E-}=R_{\Psi \backslash\left(\Gamma \cup_{\Sigma}\right)}^{A-}+\left(R_{\Sigma}^{B-} \cap \Re_{\Sigma}^{\Sigma-}\right) \text {, and } \\
& \mathfrak{N}_{\Psi \backslash \Gamma}^{\Sigma-} \cap \Re_{\Psi \backslash E}^{-}=\Re_{A^{\prime}}^{-}+\mathscr{M}_{B^{\prime}}^{B^{\prime}-} \text {. }
\end{aligned}
$$

Thus we define

$$
\begin{aligned}
V_{1}^{\prime} & =\Re_{A}^{A-}, \quad V_{2}^{\prime}=R_{\Psi}^{A-}\left(\Gamma \cup_{\Sigma}\right) \text { and } \quad V_{3}^{\prime}=\Re_{A^{\prime}}^{-} ; \\
V_{1}^{\prime \prime} & =\Re_{B}^{B-}, \quad V_{2}^{\prime \prime}=\left(R_{\Sigma}^{B-} \cap \Re_{\Sigma}^{\Sigma-}\right) \text { and } V_{3}^{\prime \prime}=\Re_{B^{\prime}}^{B^{\prime}-} ; \text { and } \\
V_{1} & =V_{1}^{\prime} \oplus V_{1}^{\prime \prime}, \quad V_{2}=V_{2}^{\prime} \oplus V_{2}^{\prime \prime} \text { and } V_{3}=V_{3}^{\prime} \oplus V_{3}^{\prime \prime} .
\end{aligned}
$$

Now every $\left.v \in \mathscr{N} T_{\Psi \backslash \Gamma}^{\Sigma}\right\rangle_{\Gamma}$ has unique decomposition

$$
v=v_{1}^{\prime}+v_{1}^{\prime \prime}+v_{2}^{\prime}+v_{2}^{\prime \prime}+v_{3}^{\prime}+v_{3}^{\prime \prime}, \quad v_{i}^{\prime} \in V_{i}^{\prime} \text { and } v_{j}^{\prime \prime} \in V_{j}^{\prime \prime}
$$

that we use without further explanation. 
If $P \subset Q \subset \Psi$ we have the bounded domain $D_{Q}^{P}=\xi^{-1} G_{Q, 0}^{P}\left(x_{0}\right)$ in $\Re_{Q}^{P-}$. Now the bounded domains in $V_{3}^{\prime}, V_{3}^{\prime \prime}$ and $V_{3}$ that serve our Siegel domains, are

(11.14a) $\quad D_{A^{\prime}}=\xi^{-1} G_{A^{\prime}, 0}\left(x_{0}\right) \subset V_{3}^{\prime}, \quad D_{B^{\prime}}^{B^{\prime}}=\xi^{-1} G_{B^{\prime}, 0}^{B^{\prime}}\left(x_{0}\right) \subset V_{3}^{\prime \prime}$, and

$$
D_{A^{\prime}} \times D_{B^{\prime}}^{B^{\prime}}=D_{E^{\prime}}^{B^{\prime}}=\xi^{-1} G_{E^{\prime}, 0}^{B^{\prime}}\left(x_{0}\right) \subset V_{3} .
$$

Note that they are the respective intersections of $V_{3}^{\prime}, V_{3}^{\prime \prime}$ and $V_{3}$ with the bounded domain $D_{\Psi \backslash E, 0}\left(x_{0}\right) \subset \Re_{\bar{\Psi} \backslash E}$.

The real forms of $V_{1}^{\prime}, V_{1}^{\prime \prime}$ and $V_{1}$ are

$$
\begin{aligned}
U_{1}^{\prime} & =\operatorname{ad}\left(c_{A}\right)\left\{g_{0} \cap \operatorname{ad}\left(c_{A}^{-1}\right) V_{1}^{\prime}\right\}, \\
U_{1}^{\prime \prime} & =\operatorname{ad}\left(c_{B}\right)\left\{g_{0} \cap \operatorname{ad}\left(c_{B}^{-1}\right) V_{1}^{\prime \prime}\right\} \quad \text { and } \\
U_{1}^{\prime} & \oplus U_{1}^{\prime \prime}=U_{1}=\operatorname{ad}\left(c_{E}\right)\left\{g_{0} \cap \operatorname{ad}\left(c_{E}^{-1}\right) V_{1}\right\} .
\end{aligned}
$$

They contain the self dual cones

(11.16a) $\mathfrak{S}_{A}=\operatorname{ad}\left(K_{A}^{A^{*}}\right) \cdot \xi^{-1}\left(x_{A, \phi}\right) \subset U_{1}^{\prime}$ where $\xi^{-1}\left(x_{A, \phi}\right)=\sum_{A e-\psi}$,

(11.16b) $\mathbb{E}_{B}=\operatorname{ad}\left(K_{B}^{B^{*}}\right) \cdot \xi^{-1}\left(x_{B, \phi}\right) \subset U_{1}^{\prime \prime}$ where $\xi^{-1}\left(x_{B, \phi}\right)=\sum_{B e-\psi}$,

$$
\mathfrak{C}_{A} \times \mathfrak{\complement}_{B}=\mathfrak{夭}_{E}=\operatorname{ad}\left(K_{E}^{E^{*}}\right) \cdot \xi^{-1}\left(x_{E, \phi}\right) \subset U_{1} \text { where }
$$

$$
\xi^{-1}\left(x_{E, \phi}\right)=\sum_{E e-\psi} \text {. }
$$

If $w \in D_{E^{\prime}}^{B^{\prime}}$ we have the conjugate-linear map $\mu(w): V_{2} \rightarrow V_{2}$ of operator norm $<1$, given by

$$
\begin{aligned}
\mu(w) v= & \operatorname{ad}(w) \cdot \operatorname{ad}\left(c_{E}^{2}\right) \cdot \tau_{u}(v)=\operatorname{ad}\left(w^{\prime}\right) \cdot \operatorname{ad}\left(c_{A}^{2}\right) \cdot \tau_{u}\left(v^{\prime}\right) \\
& +\operatorname{ad}\left(w^{\prime \prime}\right) \cdot \operatorname{ad}\left(c_{B}^{2}\right) \cdot \tau_{u}\left(v^{\prime \prime}\right) .
\end{aligned}
$$

It specifies the "bilinear plus hermitian" map $F_{w}: V_{2} \times V_{2} \rightarrow V_{1}$ by

$$
F_{w}(u, v)=-(i / 2)\left[u, \operatorname{ad}\left(c_{E}^{2}\right) \cdot \tau_{u} \cdot(I-\mu(w))^{-1} v\right] .
$$

The splitting in (11.17a) says that $\mu(w): V_{2} \rightarrow V_{2}$ is the direct sum of operators

$$
\mu\left(w^{\prime}\right): V_{2}^{\prime} \rightarrow V_{2}^{\prime} \text { and } \mu\left(w^{\prime \prime}\right): V_{2}^{\prime \prime} \rightarrow V_{2}^{\prime \prime} .
$$

Thus $(I-\mu(w))^{-1}: V_{2} \rightarrow V_{2}$ splits as the direct sum of

$$
\left(I-\mu\left(w^{\prime}\right)\right)^{-1}: V_{2}^{\prime} \rightarrow V_{2}^{\prime} \text { and }\left(I-\mu\left(w^{\prime \prime}\right)\right)^{-1}: V_{2}^{\prime \prime} \rightarrow V_{2}^{\prime \prime} .
$$

It follows that $F_{w}: V_{2} \times V_{2} \rightarrow V_{1}$ is the direct sum of maps

$$
F_{w^{\prime}}: V_{2}^{\prime} \times V_{2}^{\prime} \rightarrow V_{1}^{\prime} \text { and } F_{w^{\prime \prime}}: V_{2}^{\prime \prime} \times V_{2}^{\prime \prime} \rightarrow V_{1}^{\prime \prime}
$$


in the sense that, for $w \in D_{E^{\prime}}^{B^{\prime}}$ and $u, v \in V_{2}$,

$$
F_{w}(u, v)=F_{w^{\prime}}\left(u^{\prime}, v^{\prime}\right)+F_{w^{\prime \prime}}\left(u^{\prime \prime}, v^{\prime \prime}\right) .
$$

Carrying (11A.1) back to our situation, now

$$
\begin{aligned}
& \widetilde{S}_{\Psi \backslash(\Gamma \cup \Sigma), A}=\left\{v^{\prime} \in \mathfrak{M}_{\bar{\Psi} \backslash(\Gamma \cup \Sigma)}: v_{3}^{\prime} \in D_{A^{\prime}}\right. \text { and } \\
& \left.\operatorname{Im} v_{1}^{\prime}-F_{v_{3}^{\prime}}\left(v_{2}^{\prime}, v_{2}^{\prime}\right) \in \mathbb{夭}_{A}\right\}, \\
& \mathcal{S}_{\Sigma, B}^{\Sigma}=\left\{v^{\prime \prime} \in \mathfrak{M}_{\Sigma}^{\Sigma-}: v_{3}^{\prime \prime} \in D_{B^{\prime}}^{B^{\prime}}\right. \text { and } \\
& \left.\operatorname{Im} v_{1}^{\prime \prime}=F_{v_{3}^{\prime}}\left(v_{2}^{\prime \prime}, v_{2}^{\prime \prime}\right) \in \mathfrak{C}_{B}\right\}, \\
& \mathcal{S}_{\Psi \backslash \Gamma, E}^{\Sigma}=\left\{v \in \Re_{\Psi \backslash \Gamma}^{\Sigma-}: v_{3} \in D_{E^{\prime}}^{B^{\prime}}\right. \text { and } \\
& \left.\operatorname{Im} v_{1}-F_{v_{3}}\left(v_{2}, v_{2}\right) \in \mathfrak{C}_{E}\right\}
\end{aligned}
$$

are Siegel domains of type III that satisfy

(11.18d) $S_{\Psi \backslash \Gamma, E}^{\Sigma}=S_{\Psi \backslash(\Gamma \cup \Sigma), A} \times S_{\Sigma, B}^{B} \subset \Re_{\Psi \backslash(\Gamma \cup \Sigma)}^{\bar{D}} \oplus \mathfrak{M}_{\Sigma}^{\Sigma-}=\mathfrak{M}_{\Psi \backslash \Gamma}^{\Sigma-}$.

Furthermore, (11A.2) in our context says that

$$
\begin{gathered}
c_{A} G_{\Psi \backslash(\Gamma \cup \Sigma), 0}\left(x_{0}\right) \subset \xi\left(\Re_{\Psi \backslash(\Gamma \cup \Sigma)}\right) \text { and } \\
\xi^{-1}: c_{A} G_{\Psi \backslash(\Gamma \cup \Sigma, 0}\left(x_{0}\right) \cong S_{\Psi \backslash(\Gamma \cup \Sigma), A},
\end{gathered}
$$

(11.19b) $\quad c_{B} G_{\Sigma, 0}^{\Sigma}\left(x_{0}\right) \subset \xi\left(\Re \tau_{\Sigma}^{\Sigma-}\right)$ and $\xi^{-1}: c_{B} G_{\Sigma, 0}^{\Sigma}\left(x_{0}\right) \cong S_{\Sigma, B}^{\Sigma}$,

(11.19c) $\quad c_{E} G_{\Psi \backslash \Gamma, 0}^{\Sigma}\left(x_{0}\right) \subset \xi\left(\Re_{\Psi \backslash \Gamma}^{\Sigma-}\right)$ and $\xi^{-1}: c_{E} G_{\Psi \backslash \Gamma, 0}^{\Sigma}\left(x_{0}\right) \cong \Im_{\Psi \backslash \Gamma, E}^{\Sigma}$,

such that the analytic equivalence of $(11.19 \mathrm{c})$ is the direct product of those of (11.19a) and (11.19b). Finally, now, the coarse structure portion of Proposition 11A.3 becomes:

11.20. Proposition. Let $\Gamma$ and $\Sigma$ be disjoint subsets of $\Psi$, let $E \subset \Psi \backslash \Gamma$, and define disjoint union decompositions by

$E=A \cup B, E^{\prime}=A^{\prime} \cup B^{\prime}, \Psi \backslash(\Gamma \cup \Sigma)=A \cup A^{\prime}$ and. $\Sigma=B \cup B^{\prime}$.

Retain the notation (11.13) through (11.18). Then we have holomorphic fibre bundles

$$
\pi^{\prime}: \mathfrak{S}_{\Psi \backslash(\Gamma \cup \Sigma), A} \rightarrow D_{A^{\prime}}, \quad \pi^{\prime \prime}: \Im_{\Sigma, B}^{\Sigma} \rightarrow D_{B^{\prime}}^{B^{\prime}} \quad \text { and } \quad \pi: \mathfrak{S}_{\Psi \backslash \Gamma, E}^{\Sigma} \rightarrow D_{E^{\prime}}^{B^{\prime}}
$$

given by

$$
\pi^{\prime}\left(v^{\prime}\right)=v_{3}^{\prime}, \quad \pi^{\prime \prime}\left(v^{\prime \prime}\right)=v_{3}^{\prime \prime} \quad \text { and } \quad \pi(v)=v_{3}=v_{3}^{\prime}+v_{3}^{\prime \prime} .
$$

1. There are connected Lie groups $H_{\pi^{\prime}}, H_{\pi^{\prime \prime}}$ and $H_{\pi}$ that act transitively on the respective total spaces of $\pi^{\prime}, \pi^{\prime \prime}$ and $\pi$ by holomorphic bundle 
automorphisms. They have semidirect product structure

$$
H_{\pi^{\prime}}=G_{A^{\prime}, 0} \cdot Z_{\pi^{\prime}}, \quad H_{\pi^{\prime \prime}}=G_{B^{\prime}, 0}^{B^{\prime}} \cdot Z_{\pi^{\prime \prime}} \text { and } \quad H_{\pi}=G_{E^{\prime}, 0}^{B^{\prime}} \cdot Z_{\pi}
$$

where the first factor is equivariant for its natural transitive action on the base, and where the normal subgroups composing the second factor are the respective identity components of

$G_{\Psi \backslash(\mathrm{r} \cup \Sigma)} \cap \operatorname{ad}\left(c_{A}\right) Z_{A, \phi, 0}, \quad G_{\Sigma}^{\Sigma} \cap \operatorname{ad}\left(c_{B}\right) Z_{B, \phi, 0}$ and $G_{\Psi \backslash \Gamma}^{\Sigma} \cap \operatorname{ad}\left(c_{E}\right) Z_{E, \phi, 0}$

The latter are trivial on the base and transitive on every fibre. Finally, $H_{\pi}$ acts on $\mathfrak{S}_{\Psi \backslash \Gamma, E}^{\Sigma}=\mathfrak{S}_{\Psi \backslash(\Gamma \cup \Sigma), A} \times \mathfrak{S}_{\Sigma, B}^{\Sigma}$ as $H_{\pi^{\prime}} \times H_{\pi^{\prime \prime}}$.

2. The fibres of $\pi^{\prime}, \pi^{\prime \prime}$ and $\pi$ are holomorphically equivalent to hermitian symmetric spaces of noncompact type with respective ranks $|A|$, $|B|$ and $|E|=|A|+|B|$ and with respective tube parts $G_{A, 0}^{A}\left(x_{0}\right)$, $G_{B, 0}^{B}\left(x_{0}\right)$ and $G_{E, 0}^{E}\left(x_{0}\right)=G_{A, 0}^{A}\left(x_{0}\right) \times G_{B, 0}^{B}\left(x_{0}\right)$. The realizations of the fibres over 0 as Siegel domains of type II are

$$
\begin{aligned}
& \pi^{\prime-1}(0)=\left\{v_{1}^{\prime}+v_{2}^{\prime} \in \mathbb{M}_{A}^{A-}+\mathcal{R}_{\Psi \backslash(\Gamma \cup \Sigma)}^{A-}: \operatorname{Im} v_{1}^{\prime}-F_{0}\left(v_{2}^{\prime}, v_{2}^{\prime}\right) \in \mathfrak{\mho}_{A}\right\}, \\
& \pi^{\prime \prime-1}(0)=v\left\{\begin{array}{l}
\prime \prime \\
1
\end{array}\right. \\
& \left.\operatorname{Im} v_{1}^{\prime \prime}-F_{0}\left(v_{2}^{\prime \prime}, v_{2}^{\prime \prime}\right) \in \mathbb{C}_{B}\right\}, \\
& \pi^{-1}(0)=\pi^{\prime-1}(0) \times \pi^{\prime \prime-1}(0)=\left\{v \in V_{1} \oplus V_{2}: \operatorname{Im} v_{1}-F_{0}\left(v_{2}, v_{2}\right) \in \mathfrak{C}_{E}\right\} .
\end{aligned}
$$

3. The bundles $\pi^{\prime}, \pi^{\prime \prime}$ and $\pi$ are real-analytically trivial. Let $\left\{\Delta_{k}^{\prime}\right\}$ be the root systems of the simple ideals of $\mathcal{G}_{\Psi} \backslash(\Gamma \cup \Sigma),\left\{\Delta_{l}^{\prime \prime}\right\}$ the root systems of the simple ideals of $\mathrm{g}_{\mathrm{\Sigma}}^{\mathrm{V}}$. Then

(3a) $\pi^{\prime}$ is holomorphically trivial if and only if $A$ is a union of sets of the form $[\Psi \backslash(\Gamma \cup \Sigma)] \cap \Delta_{k}^{\prime}$,

(3b) $\pi^{\prime \prime}$ is holomorphically trivial if and only if $B$ is a union of sets of the form $\Sigma \cap \Delta . .^{\prime \prime}$, and

(3c) $\pi$ is holomorphically trivial if and only if both $\pi^{\prime}$ and $\pi^{\prime \prime}$ are holomorphically trivial.

We come to the final step of the decomposition of $G_{0}\left(x_{\Gamma, \Sigma}\right)$. Recall $\beta: G_{0}\left(x_{\Gamma, \Sigma}\right) \rightarrow K\left(x_{\Gamma, \Sigma}\right)$ with $\beta^{-1} k\left(x_{\Gamma, \Sigma}\right)=k \cdot G_{\Psi \backslash \Gamma, 0}^{\Sigma}\left(x_{\Gamma, \Sigma}\right)$. Proposition 11.20 fibres

$$
\beta^{-1}\left(x_{\Gamma, \Sigma}\right)=G_{\Psi \backslash \Gamma, 0}^{\Sigma}\left(x_{\Gamma, \Sigma}\right) \rightarrow G_{E^{\prime}, 0}^{B^{\prime}}\left(x_{\Gamma, \Sigma}\right)
$$

by

$$
c_{\Gamma} c_{\Sigma}^{2} c_{E}^{-1} \xi(v) \rightarrow c_{\Gamma} c_{\Sigma}^{2} \xi(\pi v) \quad \text { for every } v \in S_{\Psi}^{2}
$$


As seen from the case where the unit disc is fibered over one of its (one point ) boundary components, $G_{E^{\prime}, 0}^{B^{\prime}}\left(x_{\Gamma, \Sigma}\right)$ is generally not $K_{\Psi}^{\Sigma} \backslash \Gamma^{-}$ stable, so we cannot just extend (11.21) to $G_{0}\left(x_{\Gamma, \Sigma}\right)$ by $K$-equivariance. That problem is avoided by use of a measurable, a.e. real analytic, "section" of the principal $K_{\Psi \backslash \Gamma}^{\Sigma}$-bundle $K \rightarrow K / K_{\Psi \backslash \Gamma}^{\Sigma}$.

Let $V_{\Psi \backslash \Gamma}^{\Sigma}$ denote the orthocomplement of $\mathcal{K}_{\Psi \backslash \Gamma}^{\Sigma}$ in $\kappa$; define $V_{\Psi \backslash \Gamma}^{\Sigma}=\exp \left(v_{\Psi \backslash \Gamma}^{\Sigma}\right) \subset K$. Then every $k \in K$ has expression $k=v k^{\prime}$, $v \in V_{\Psi \backslash \Gamma}^{\Sigma}$ and $k^{\prime} \in K_{\Psi \backslash \Gamma}^{\Sigma}$, but that factorization need not be unique. To approach uniqueness, let $K / K_{\Psi \backslash \Gamma}^{\Sigma}$ carry a riemannian metric induced by a bi-invariant metric on $K$, so the exponential map

$$
\exp _{\vartheta}: v_{\Psi \backslash \Gamma}^{\Sigma} \rightarrow K / K_{\Psi \backslash \Gamma}^{\Sigma} \quad \text { by } \exp _{\vartheta}(v)=\exp (v) \cdot \vartheta
$$

where $\mathcal{O}$ is the base point. Now let $W_{\Psi \backslash \Gamma}^{\Sigma}$ consist of all $v \in \mathcal{V}_{\Psi \backslash \Gamma}^{\Sigma}$ such that $\exp _{\theta}(t v), 0 \leqq t \leqq 1$, is the unique minimizing geodesic from $\theta$ to $\exp _{\mathcal{O}}(v)$. Define $W_{\Psi \backslash \Gamma}^{\Sigma}=\exp \left(W_{\Psi \backslash \Gamma}^{\Sigma}\right) \subset V_{\Psi \backslash \Gamma}^{\Sigma}$. Then

(11.22a) $W_{\Psi \backslash \Gamma}^{\Sigma}(\mathcal{\theta})$ is a dense open subset of $K / K_{\Psi}^{\Sigma} \backslash$, and

(11.22b) exp: $W_{\Psi \backslash \Gamma}^{\Sigma} \rightarrow W_{\Psi \backslash \Gamma}^{\Sigma}(\mathcal{\theta})$ is a diffeomorphism.

That gives us a "section" $s: K / K_{\Psi \backslash \Gamma}^{\Sigma} \rightarrow K$ by

(11.22c) $s\left(\exp _{\Theta}(w)\right)=\exp (w) \in W_{\Psi \backslash \Gamma}^{\Sigma} \quad$ for $w \in w_{\Psi \backslash \Gamma}^{\Sigma}$,

(11.22d) $s(k \cdot \mathcal{\theta}) \in k K_{\Psi \backslash \Gamma}^{\Sigma} \quad$ arbitrary for $k \cdot \mathcal{\theta} \notin W_{\Psi \backslash \Gamma}^{\Sigma}(\mathcal{\theta})$.

Although $s$ may be discontinuous, it is real analytic on the dense open subset $W_{\Psi \backslash \Gamma}^{\Sigma}(\theta) \subset K / K_{\Psi \backslash \Gamma}^{\Sigma}$ whose complement has measure zero.

11.23. TheOREM. Let $\Gamma$ and $\Sigma$ be disjoint subsets of $\Psi$, let $E \subset \Psi \backslash \Gamma$, and consider disjoint unions

$E=A \cup B, \quad E^{\prime}=A^{\prime} \cup B^{\prime}, \quad \Psi \backslash(\Gamma \cup \Sigma)=A \cup A^{\prime}, \quad \Sigma=B \cup B^{\prime}$. If $k \in K$, define

$$
\gamma_{k}^{A, B}: k \cdot G_{\Psi \backslash \Gamma, 0}^{\Sigma}\left(x_{\Gamma, \Sigma}\right) \rightarrow k \cdot G_{E^{\prime}, 0}^{B^{\prime}}\left(x_{\Gamma, \Sigma}\right)
$$

by

$$
\gamma_{k}^{A, B}\left(k c_{\Gamma} c_{\Sigma}^{2} c_{E}^{-1} \xi v\right)=k c_{\Gamma} c_{\Sigma}^{2} \xi(\pi v) \quad \text { for } v \in \mathfrak{S}_{\Psi \backslash \Gamma, E}^{\Sigma} .
$$

Retain the notation (11.22) and define

$$
\gamma^{\Lambda, B}: G_{0}\left(x_{\Gamma, \Sigma}\right) \rightarrow s\left(K / K_{\Psi \backslash \Gamma}^{\Sigma}\right) \cdot G_{E^{\prime}, 0}^{B^{\prime}}\left(x_{\Gamma, \Sigma}\right)
$$


by

$$
\left.\gamma^{A, B}\right|_{k G_{\Psi \backslash \Gamma, 0}^{\Sigma}\left(x_{\Gamma, \Sigma}\right)}=\underset{\gamma_{s(k \cdot 0)}^{A, B}}{ }
$$

1. Given $k \in K, \gamma_{k}^{A, B}$ is a holomorphic fibre bundle that is equivalent to the bundle $\pi: \mathfrak{S}_{\Psi \backslash \Gamma, E}^{\Sigma} \rightarrow D_{E^{\prime}}^{B^{\prime}}$ of Proposition 11.20 under the maps:

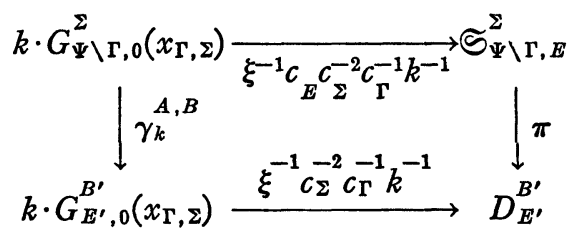

2. $\boldsymbol{\gamma}^{A, B}$ is a well-defined, but not necessarily continuous, "fibre bundle" whose fibres coincide with those of its restrictions $\gamma_{s(k \cdot 0)}^{A, B}$, and which is real analytic over $W_{\Psi \backslash \Gamma}^{\Sigma} \cdot G_{E^{\prime}, 0}^{B^{\prime}}\left(x_{\Gamma, \Sigma}\right)$.

3. The fibration $\beta: G_{0}\left(x_{\Gamma, \Sigma}\right) \rightarrow K\left(x_{\Gamma, \Sigma}\right)$ of Theorem 11.8 comes from $\gamma^{A, B}$ by

$$
\beta=\left\{\beta \cdot\left(\gamma^{A, B}\right)^{-1}\right\} \cdot \gamma^{A, B} \text { where }\left\{\beta \cdot\left(\gamma^{A, B}\right)^{-1}\right\} \cdot k G_{E^{\prime}}^{B^{\prime}}\left(x_{\Gamma, \Sigma}\right)=k\left(x_{\Gamma, \Sigma}\right) .
$$

Proof. Statement (1) is immediate from Proposition 11.20. $\gamma_{k}^{A, B}$ sends the $\beta$-fibre $k \cdot G_{\Psi \backslash \Gamma, 0}^{\Sigma}\left(x_{\Gamma, \Sigma}\right)=s(k \cdot \theta) \cdot G_{\Psi \backslash \Gamma, 0}^{\Sigma}\left(x_{\Gamma, \Sigma}\right)$ over $k\left(x_{\Gamma, \Sigma}\right)$ $=s(k \cdot \theta)\left(x_{\Gamma, \Sigma}\right)$ into its hermitian symmetric subspace $k \cdot G_{E^{\prime}, 0}^{B^{\prime}}\left(x_{\Gamma, \Sigma}\right)$. As $\beta$ is well defined, (3) follows.

We check that $\gamma^{A, B}$ is well defined. Let $x \in G_{0}\left(x_{\Gamma, \Sigma}\right)$. Suppose $\beta(x)=k\left(x_{\Gamma, \Sigma}\right), \quad k \in K$. Then $x \in \beta^{-1} k(x)=s(k \cdot \theta) \cdot G_{\Psi \backslash \Gamma, 0}^{\Sigma}\left(x_{\Gamma, \Sigma}\right)$, so $x=s(k \cdot \theta) g\left(x_{\Gamma, \Sigma}\right)$ for some $g \in G_{\Psi \backslash \Gamma, 0}^{\Sigma}$. Now, by definition, $\gamma^{A, B}(x)$ $=\gamma_{s(k \cdot \theta)}^{A, B}(x)=s(k \cdot \theta) c_{\Gamma} c_{\Sigma} \xi(\pi v)$ where $v \in \Im_{\Psi, \Sigma}^{\Sigma} \backslash$ such that $g\left(x_{\Gamma, \Sigma}\right)$ $=c_{\Gamma} c_{\Sigma}^{2} c_{E}^{-1} \xi(v)$. That procedure is unique, first because $k$ is specified modulo $K_{\Psi \backslash \Gamma}$ because $\beta$ is well defined, and then because $k$ is specified modulo $K_{\Psi \backslash \Gamma}^{\Sigma}$ by Lemma 11.4 .

$\left(\gamma^{A, B}\right)^{-1}\left(s(k \cdot \mathcal{\theta})\left(x_{\Gamma, \Sigma}\right)\right)=\left(\gamma_{s(k \cdot \mathcal{A})}^{A, B}\right)^{-1}\left(s(k \cdot \mathcal{\theta})\left(x_{\Gamma, \Sigma}\right)\right)$ by construction and well definition of $\beta \cdot\left(\gamma^{A, B}\right)^{-1}$.

Over $W_{\Psi \backslash \Gamma}^{\Sigma} \cdot G_{E^{\prime}, 0}^{B^{\prime}}\left(x_{\Gamma, \Sigma}\right), \gamma^{A, B}$ is given by

$$
\boldsymbol{\gamma}^{A, B}\left(k c_{\Gamma} c_{\Sigma}^{2} c_{E}^{-1} \xi(v)\right)=k c_{\Gamma} c_{\Sigma}^{2} \xi(\pi v), \quad k \in W_{\Psi \backslash \Gamma}^{\Sigma} \quad \text { and } \quad v \in \widetilde{S}_{\Psi \backslash \Gamma, E}^{\Sigma}
$$

That is real analytic. Q.E.D.

We now have the triple fibration 


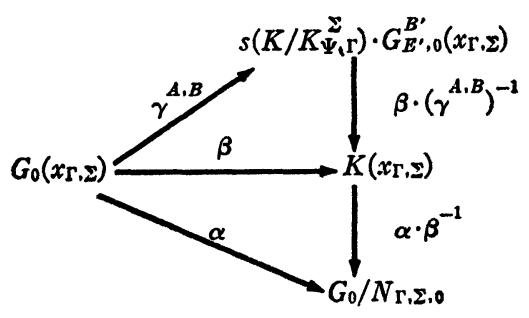

of an arbitrary orbit $G_{0}\left(x_{\Gamma, \Sigma}\right) \subset X$ as described at the beginning of this $\$ 11$.

Appendix to $\$ 11$-Siegel domains. We first recall the concepts of cone and Siegel domain.

Let $U$ be a real vector space with a positive definite inner product $\langle$,$\rangle . Then a self dual cone in U$ means a subset $\mathbb{\subseteq} \subset$ such that

$$
\mathfrak{C}=\{u \in U: \text { if } 0 \neq v \in \mathbb{\mathfrak { C }} \text { then }\langle u, v\rangle>0\} .
$$

It is automatic that a self dual cone is a cone (nonempty, and $u \in \mathbb{C}$ and $r>0$ imply $r u \in(\mathfrak{E})$, is open, is convex, and does not contain a straight line. "Domain of positivity" is another term for self dual cone.

Let $\mathbb{S} \subset U$ be a convex open cone that does not contain a straight line. Define $V=U+i U$ complexification; if $v \in V$ than $v=(\operatorname{Re} v)$ $+i(\operatorname{Im} v)$ with $\operatorname{Re} v$ and $\operatorname{Im} v$ in $U$. The tube domain over $\mathbb{E}$ is defined to be the set $\{v \in V: \operatorname{Im} v \in \mathbb{E}\}$. The Poincaré and Siegel upper half planes are examples. By Siegel domain of type I we mean a tube domain over a convex open cone that does not contain a straight line. A hermitian symmetric space is of tube type if it is holomorphically diffeomorphic to a Siegel domain of type I; in that case the cone is self dual. A standard classification result says that a hermitian symmetric space is of tube type if and only if it is a product, each of whose factors is one of

$$
\begin{array}{ll}
\mathrm{SU}(m, m) / \mathrm{S}(\mathbf{U}(m) \times \mathbf{U}(m)), & m \geqq 1 ; \text { or } \\
\mathbf{S O} *(4 m) / \mathbf{U}(2 m), & m \geqq 2 ; \text { or } \\
\mathrm{Sp}(m ; \mathbf{R}) / \mathbf{U}(m), & m \geqq 1 ; \text { or } \\
\mathbf{S O}(2, m) / \mathbf{S O}(2) \times \mathbf{S O}(m), & m \geqq 1 ; \text { or } \\
\mathbf{E}_{7, \text { index-25 }} / \mathbf{E}_{8} \cdot \mathbf{S O}(2) . &
\end{array}
$$

Let $V_{1}$ and $V_{2}$ be complex vector spaces, $U_{1}$ a real form of $V_{1}$, c $\subset U_{1}$ a cone as above, and $F: V_{2} \times V_{2} \rightarrow V_{1}$ an hermitian map that is positive definite in the sense 


$$
0 \neq v_{2} \in V_{2} \quad \text { implies } 0 \neq F\left(v_{2}, v_{2}\right) \in \overline{\mathfrak{c}} \text {. }
$$

That data defines the domain

$$
\left\{\left(v_{1}, v_{2}\right) \in V_{1} \oplus V_{2}: \operatorname{Im} v_{1}-F\left(v_{2}, v_{2}\right) \in \mathbb{E}\right\} .
$$

Those domains are the Siegel domains of type II. Siegel domains of type I are the special case $V_{2}=0$. Every hermitian symmetric space of noncompact type has a standard realization (Korányi-Wolf [2]) as a Siegel domain of type II, and that Siegel domain is of type I if and only if the hermitian symmetric space is of tube type; the cone is self dual.

Let $V_{1}, V_{2}$ and $V_{3}$ be complex vector spaces, $U_{1}$ a real form of $V_{1}$, $\subseteq \subset U_{1}$ a cone as above, and $D \subset V_{3}$ a bounded domain. Suppose further that, for every $w \in D$, we have

$$
\begin{aligned}
& F_{w}^{h}: V_{2} \times V_{2} \rightarrow V_{1} \text { hermitian and } \\
& F_{w}^{b}: V_{2} \times V_{2} \rightarrow V_{1} \text { bilinear }
\end{aligned}
$$

depending smoothly on $w \in D$, such that

$$
F_{0}^{b}=0 \text { and } F_{0}^{h} \text { is positive definite relative to } \mathfrak{E} \text {. }
$$

Then we define

$$
F_{w}: V_{2} \times V_{2} \rightarrow V_{1} \quad \text { by } F_{w}=F_{w}^{h}+F_{w}^{b} .
$$

Assume that for every $w \in D, F_{w}$ is nondegenerate in the sense that each of $F_{w}\left(v, V_{2}\right)=0$ and $F_{w}\left(V_{2}, v\right)=0$ implies $v=0$. Then the domain

$$
\left\{\left(v_{1}, v_{2}, v_{3}\right) \in V_{1} \oplus V_{2} \oplus V_{3}: v_{3} \in D \text { and } \operatorname{Im} v_{1}-\operatorname{Re} F_{v_{3}}\left(v_{2}, v_{2}\right) \in \mathbb{E}\right\}
$$

is called a Siegel domain of type III with base $D$. Siegel domains of type II are the special case $V_{3}=0$, i.e. the case where the base is reduced to a point.

In $[15,87]$ Wolf and Korányi describe $X_{0}=G_{0}\left(x_{0}\right)$ as a Siegel domain of type III in $\mathfrak{N}^{-}$whose base is the bounded domain in $\Re_{\bar{\Psi} \backslash E}$ equivalent to an arbitrary boundary component $G_{\Psi \backslash E, 0}\left(x_{E, \phi}\right)$ of $X_{0}$. That description involves the holomorphic injection

$$
\xi: \Re^{-} \rightarrow X \quad \text { by } \xi(v)=\exp (v) \cdot x_{0}
$$

of $\Re C^{-}$as a dense open subset of $X$, and is given in our notation as follows.

First note $\mathfrak{N}^{-}=\mathfrak{N}_{\Psi}^{E-}+{\Re_{\Psi}^{E-}}^{E-}=\mathfrak{N}_{\bar{\Psi} \backslash E}+\mathfrak{N}_{E}^{E-}+{Q_{\Psi}^{E-}}^{E-}$ direct. Now if $v \in \Re^{-}$, we will decompose

$$
v=v_{1}+v_{2}+v_{3} \quad \text { with } v_{1} \in \mathfrak{M}_{E}^{E-}, v_{2} \in \mathcal{R}_{\Psi}^{E-}, v_{3} \in \mathfrak{M}_{\bar{\Psi} \backslash E}^{-} .
$$


Our bounded domain in $\mathfrak{T}_{\bar{\Psi} \backslash E}^{-}$is

$$
D_{\Psi \backslash E}=\xi^{-1} G_{\Psi \backslash E, 0}\left(x_{0}\right) .
$$

The real form of $\operatorname{Tr}_{E}^{E-}$ is $\operatorname{ad}\left(c_{E}\right)\left\{g_{0} \cap \operatorname{ad}\left(c_{E}^{-1}\right) \mathscr{T}_{E}^{E-}\right\}$, and the positive definite inner product on that real form is induced by the positive definite hermitian inner product $(u, v)=-\langle u, \sigma \tau v\rangle$ on $g$. The self dual cone $\mathfrak{S}_{E} \subset \operatorname{ad}\left(c_{E}\right)\left\{\mathcal{G}_{0} \cap \operatorname{ad}\left(c_{E}^{-1}\right) \mathfrak{T}_{E}^{E-}\right\}$ requires some notation. If $F \subset \Psi$ we have $K_{F}=K \cap G_{F, 0}$ and the fixed point set $K_{F}^{E}$ of $\operatorname{ad}\left(c_{E}^{4}\right)$ on $K_{F}$; now $\operatorname{ad}\left(c_{E}^{2}\right)$ restricts to an involutive automorphism of $\varkappa_{F}^{E}$, and that defines an algebra

$$
\mathfrak{K}_{F}^{E^{*}}=\left\{v \in \mathcal{K}_{F}^{E}: \operatorname{ad}\left(c_{E}^{2}\right) v=v\right\}+i\left\{v \in \mathcal{K}_{F}^{E}: \operatorname{ad}\left(c_{E}^{2}\right) v=-v\right\} .
$$

Its analytic group is denoted $K_{F}^{E^{*}}$. Now the cone is given, in our notation, by

$$
\mathfrak{S}_{E}=\operatorname{ad}\left(K_{E}^{E^{*}}\right) \cdot \xi^{-1}\left(x_{E, \phi}\right) \text { and } \xi^{-1}\left(x_{E, \phi}\right)=\sum_{E} e_{-\psi} .
$$

If $w \in \mathscr{T}_{\Psi \backslash E}^{-}$then $\mu(w): \mathbb{R}_{\Psi}^{E-} \rightarrow R_{\Psi}^{E-}$ is defined by $\mu(w) v=\operatorname{ad}(w)$ $\cdot \operatorname{ad}\left(c_{E}^{2}\right) \cdot \sigma \tau(v)$; it has operator norm $<1$ if and only if $w \in D_{\Psi \backslash E}$. Thus if $w \in D_{\Psi \backslash E}, I+\mu(w)$ is invertible and we have $F_{w}: Q_{\Psi}^{E-} \times Q_{\Psi}^{E-}$ $\rightarrow \mathfrak{T l}_{E}^{E-}$ by

$$
F_{w}(u, v)=-(i / 2)\left[u, \operatorname{ad}\left(c_{E}^{2}\right) \cdot \sigma \tau \cdot(I+\mu(w))^{-1} v\right] .
$$

The main result of $[15, \S 7]$ says first that

(11A.1) $\mathfrak{S}_{E}=\left\{v \in \mathfrak{T l}^{-}: v_{3} \in D_{\Psi \backslash E}\right.$ and $\left.\operatorname{Im} v_{1}-\operatorname{Re} F_{v_{2}}\left(v_{2}, v_{2}\right) \in \mathfrak{S}_{E}\right\}$ is a Siegel domain of type III with base $D_{\Psi \backslash E}$, and second that

$$
c_{E} X_{0} \subset \xi\left(\Re^{-}\right) \text {and } \xi^{-1}: c_{E} X_{0} \cong \mathfrak{S}_{E} \text {. }
$$

In proving the results stated above, Wolf and Korányi use certain symmetry properties

$$
\begin{aligned}
\operatorname{ad}(k) F_{w}(u, v) & =F_{\operatorname{ad}(k) w}(\operatorname{ad}(k) u, \operatorname{ad}(k) v) \quad \text { for } k \in K_{\Psi}^{E^{*}}, \\
F_{w}(u,(I+\mu(w)) v) & =F_{0}(u, v) \quad \text { and } \quad F_{0}(u, \mu(w) v)=F_{0}(v, \mu(w) u)
\end{aligned}
$$

of the $F_{w}$ and rely on the $\xi^{-1}$-equivariant actions of $\operatorname{ad}\left(c_{E}\right) Z_{E, \phi, 0}$ and $\operatorname{ad}\left(c_{E}\right) N_{E, \phi, 0}$ (cf.(10.10)) from the natural action on $c_{E} X_{0}$ to the (defined by equivariance) action on $\mathfrak{T}-$. For if $N^{E}$ denotes the common unipotent radical of those groups,

$$
N^{E}=\left\{\operatorname{ad}\left(c_{E}\right) N_{E, \phi, 0}\right\}^{u}=\left\{\operatorname{ad}\left(c_{E}\right) Z_{E, \phi, 0}\right\}^{u},
$$


then $\left[15\right.$, Theorem 6.8] $N^{E}$ is 2-step nilpotent, its Lie algebra $\Re^{E}$ has complexification

$$
\mathfrak{\Re}^{E C}=\operatorname{ad}\left(c_{E}\right) \mathfrak{T}_{E, \phi}^{u}=\operatorname{ad}\left(c_{E}\right) \mathbb{Z}_{E, \phi}^{u}=\left(Q_{\Psi}^{E_{+}}+\mathfrak{R}_{\Psi}^{E_{-}}\right)+\mathfrak{N}_{E}^{E-},
$$

and $\operatorname{ad}\left(c_{E}\right) Z_{E, \phi, 0}$ and $\operatorname{ad}\left(c_{E}\right) N_{E, \phi, 0}$ have respective identity components

$$
\left\{L_{E, \phi, 0} \cdot K_{E}^{E^{*}}\right\} \cdot N^{E} \quad \text { and } \quad\left\{G_{\Psi \backslash E, 0} \cdot L_{E, \phi, 0} \cdot K_{E}^{E^{*}}\right\} \cdot N^{E} ;
$$

here we recall $\mathcal{S}_{\Psi, 0}^{E}=\mathcal{G}_{\Psi \backslash E, 0} \oplus \mathfrak{L}_{E, \phi, 0} \oplus \mathcal{G}_{E, 0}^{E}, \mathfrak{L}_{E, \phi, 0} \subset \mathcal{K}$, so that

$$
K_{\Psi}^{E^{*}}=K_{\Psi \backslash E} \cdot L_{E, \phi, 0} \cdot K_{E}^{E^{*}} .
$$

The action of $K_{\Psi}^{E *}$ on $\Re^{-}$is induced by ad ${ }_{G}$; it preserves each of $\Re_{E}^{E-}$, $\mathcal{R}_{\Psi}^{E-}$ and $\Re_{\bar{\Psi} \backslash E}$, the real form specified of $\Re_{E}^{E-}$ and the cone $\mathfrak{C}_{E}$ there, the bilinear maps $F_{w}$ and the domain $D_{\Psi \backslash E}$, and thus also the Siegel domain $\mathfrak{S}_{E}$. The action of $N^{E}$ on $\mathfrak{T}-$ is based on the unique representation of every $g \in N^{E}$ in the form $\exp \left(u+\left(I-\operatorname{ad}\left(c_{E}^{2}\right) \sigma \tau\right) v\right)$ with $u \in \mathfrak{N}_{\mathbb{E}}^{E-}$ and $v \in \mathcal{R}_{\Psi}^{E-} ;$ if

$$
f: R_{\Psi}^{E-} \times \Re_{\Psi \backslash E}^{-} \rightarrow R_{\Psi}^{E-} \text { by }(v, w) \rightarrow f_{v}(w)=v+\mu(w) v,
$$

then $N^{E}$ acts on $\Re^{-}$by

$$
g(e)=e+u+f_{v}\left(e_{3}\right)+2 i F_{e_{3}}\left(e_{2}, f_{v}\left(e_{3}\right)\right)+i F_{e_{3}}\left(f_{v}\left(e_{3}\right), f_{v}\left(e_{3}\right)\right) ;
$$

that action preserves the Siegel domain $\mathfrak{S}_{E}$. The action of $G_{\Psi \backslash E, 0}$ on $\mathfrak{m}-$ or $\mathfrak{S}_{E}$ is not made explicit in [15], but the action of $K_{\Psi \backslash E} \subset K_{\Psi}^{E *}$ is described above, and $G_{\Psi \backslash E, 0}$ acts on $D_{\Psi \backslash E}$ in the natural fashion.

11A.3. Proposition. Let $\mathfrak{S}_{E}$ be the Siegel domain (11A.1) with base $D_{\Psi \backslash E}$ that is holomorphically equivalent $\left(b y c_{E}^{-1} \cdot \xi\right)$ to $X_{0}$. Define

$$
\pi: \mathfrak{S}_{E} \rightarrow D_{\Psi \backslash E} \text { by } \pi(v)=v_{3} .
$$

1. $\pi: \Im_{E} \rightarrow D_{\Psi \backslash E}$ is a holomorphic fibre bundle.

2. The identity component $\left\{G_{\Psi \backslash E, 0} \cdot L_{E, \phi, 0} \cdot K_{E}^{E *}\right\} \cdot N^{E}$ of $\operatorname{ad}\left(c_{E}\right) N_{E, \phi, 0}$ acts transitively on $\mathfrak{S}_{E}$ by holomorphic bundle automorphisms, with $G_{\Psi \backslash E, 0}$ transitive on the base and the identity component $\left\{L_{E, \phi, 0} \cdot K_{E}^{E *}\right\} \cdot N^{E}$ of $\operatorname{ad}\left(c_{E}\right) Z_{E, \phi, 0}$ trivial on the base and transitive on every fibre.

3. The fibre $\pi^{-1}(0)$ is the Siegel domain $\left\{v \in \mathbb{M}_{E}^{E-}+\mathfrak{R}_{\Psi}^{E-}:\right.$ Im $v_{1}$ $\left.-F_{0}\left(v_{2}, v_{2}\right) \in \mathfrak{\complement}_{E}\right\}$ of type II homogeneous under $\left\{L_{E, \phi, 0} \cdot K_{E}^{E *}\right\} \cdot N^{E}$, and it is holomorphically equivalent to a hermitian symmetric space of noncompact type that has rank $|E|$ and tube part $G_{E, 0}^{E}\left(x_{0}\right)$. 
4. The bundle $\pi: \Im_{E} \rightarrow D_{\Psi \backslash E}$ is real-analytically trivial, and it is holomorphically trivial if and only if $E$ is a union of sets $\Psi \cap \Delta^{k}$ where the $\Delta^{k}$ are the root systems of the simple ideals $\mathrm{G}^{k}$ of $\mathrm{G}$.

Proof. Let $e=e_{1}+e_{2}+e_{3} \in \widetilde{S}_{E}$ and represent $g \in N^{E}$ in the form $\exp \left(u+\left(I-\operatorname{ad}\left(c_{E}^{2}\right) \sigma \tau\right) v\right)$ with $u \in \Re_{E}^{E-}$ and $v \in \mathcal{Q}_{\Psi}^{E-}$. Let $e^{\prime}=g(e)$; then

$$
e^{\prime}=e+u+f_{v}\left(e_{3}\right)+2 i F_{e_{3}}\left(e_{2}, f_{v}\left(e_{3}\right)\right)+i F_{e_{3}}\left(f_{v}\left(e_{3}\right), f_{v}\left(e_{3}\right)\right) \text {, }
$$

so $e_{3}^{\prime}=e_{3}, e_{2}^{\prime}=e_{2}+f_{v}\left(e_{3}\right)$ and

$$
e_{1}^{\prime}=e_{1}+u+2 i F_{e_{3}}\left(e_{2}, f_{v}\left(e_{3}\right)\right)+i F_{e_{3}}\left(f_{v}\left(e_{3}\right), f_{v}\left(e_{3}\right)\right) \text {. }
$$

Thus the action of $N^{E}$ on $\Im_{E}$ preserves every $\pi$-fibre. If $k \in L_{E, \phi, 0} \cdot K_{E}^{E *}$, then $k(e)=\operatorname{ad}(k) e, \operatorname{so} k(e)_{3}=\operatorname{ad}(k) e_{3}=e_{3}$. Thus the action of $L_{E, \phi, 0} \cdot K_{E}^{E *}$, on $\mathfrak{S}_{E}$ preserves every $\pi$-fibre. Define

$$
v=-\left(I+\mu\left(e_{3}\right)\right)^{-1} e_{2} \text { and } g=\exp \left(\left(I-\operatorname{ad}\left(c_{E}^{2}\right) \sigma \tau\right) v\right) \in N^{E} .
$$

Then $g(e)=e_{1}^{\prime}+0+e_{3}$. Now define

$$
u=-\operatorname{Re} e_{1}^{\prime} \text { and } g^{\prime}=\exp (u) \in N^{E} \text {. }
$$

Then $g^{\prime} g(e)=e_{1}^{\prime \prime}+0+e_{3}$ with $i e_{1}^{\prime \prime}$ real. In fact $g^{\prime} g(e) \in \mathcal{S}_{E}$ gives us $\operatorname{Im} e_{1}^{\prime \prime}-\operatorname{Re} F_{e_{2}}(0,0)=\operatorname{Im} e_{1}^{\prime \prime} \in \mathfrak{C}_{E}$. Thus there exists $k \in K_{E}^{E^{*}}$ such that $\operatorname{ad}(k) \cdot \operatorname{Im} e_{1}^{\prime \prime}=\xi^{-1}\left(x_{E, \phi}\right)$. Now $k g^{\prime} g \in K_{E}^{E^{*}} \cdot N^{E}$ has the property that

$$
\left(k g^{\prime} g\right)(e)=i \xi^{-1}\left(x_{E, \phi}\right)+e_{3} .
$$

We have proved that the $\pi$-fibres are just the orbits of $\left\{L_{E, \phi, 0} \cdot K_{E}^{E^{*}}\right\} \cdot N^{E}$ on $\mathfrak{S}_{E}$.

Let $g \in G_{\Psi \backslash E, 0}$. Then $g$ normalizes $\left\{L_{E, \phi, 0} \cdot K_{E}^{E^{*}}\right\} \cdot N^{E} \quad$ in $\left\{G_{\Psi \backslash E, 0} \cdot L_{E, \phi, 0} \cdot K_{E}^{E^{*}}\right\} \cdot N^{E}$, hence permutes its orbits on $\subseteq_{E}$. Now $g$ is a $\pi$-fibre map of $\mathfrak{S}_{E}$. Thus (2) is proved subject to (1) being demonstrated.

Let $Q$ denote the identity component of $\operatorname{ad}\left(c_{E}\right) N_{E, \phi, 0}, Y$ its isotropy subgroup at $i \xi^{-1}\left(x_{E, \phi}\right) \in \Im_{E}$, and $Z$ the subgroup preserving $\pi^{-1}(0)$. Then $Y \subset Z=\left\{K_{\Psi \backslash E} \cdot L_{E, \phi, 0} \cdot K_{E}^{E^{*}}\right\} \cdot N^{E}=K_{\Psi}^{E^{*}} \cdot N^{E}$ and $\pi$ is projection $Q / Y \rightarrow Q / Z$ by $q Y \rightarrow q Z$. Thus $\pi$ is a differentiable fibre bundle with structure group $Z$. But $\pi$ visibly is a holomorphic map, and the fibre over $g(0), g \in G_{\Psi \backslash E, 0}$, is the complex submanifold

$$
g \cdot \pi^{-1}(0)=g \cdot\left\{v \in \Re_{E}^{E-}+R_{\Psi}^{E-}: \operatorname{Im} v_{1}-F_{0}\left(v_{2}, v_{2}\right) \in \mathfrak{E}_{E}\right\}
$$

of $\Im_{E}$. As $Z$ acts by holomorphic transformations of $\pi^{-1}(0)$, it follows that $\pi: \widetilde{S}_{E} \rightarrow D_{\Psi \backslash E}$ is a holomorphic fibre bundle. That completes the proof of (1) and (2). 
Every $\pi$-fibre is of the form $g \cdot \pi^{-1}(0), g \in G_{\Psi \backslash E, 0}$, and $G_{\Psi \backslash \mathbb{\Psi}, 0}$ normalizes the transitive group $\left\{L_{E, \phi, 0} \cdot K_{E}^{E *}\right\} \cdot N^{E}$ of transformations of the fibre; thus an arbitrary $\pi$-iibre has the same properties as $\pi^{-1}(0)$ as complex homogeneous space of $\left\{L_{E, \phi, 0} \cdot K_{E}^{E *}\right\} \cdot N_{E}^{E}$. Now we observe

$$
\pi^{-1}(0)=\left\{v \in \Re_{E}^{E-}+\mathfrak{R}_{\Psi}^{E-}: \operatorname{Im} v_{1}-F_{0}\left(v_{2}, v_{2}\right) \in \mathfrak{夭}_{E}\right\}
$$

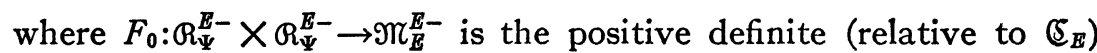
hermitian map given by

$$
F_{0}(u, v)=-(i / 2)\left[u, \operatorname{ad}\left(c_{E}^{2}\right) \sigma \tau v\right] .
$$

Thus $\pi^{-1}(0)$, hence every fibre, is a homogeneous Siegel domain of type II. Comparison with $[2, \S 6]$ suggests that it should be symmetric, and we prove that symmetry as follows.

Observe that $\left\{v \in \pi^{-1}(0): v_{2}=0\right\}$ is the tube domain over the self dual cone $\mathfrak{C}_{E}$; in fact that is the realization of $G_{E, 0}^{\mathbb{E}, 0}\left(x_{0}\right)$ as a Siegel domain of type I. Thus there is an analytic automorphism $\delta$ of order 2 on that tube domain with $i \xi^{-1}\left(x_{E, \phi}\right)$ as the only fixed point. Given $e \in \pi^{-1}(0)$, define

$$
v=-e_{2} \text { and } g_{e}=\exp \left(\left(I-\operatorname{ad}\left(c_{E}^{2}\right) \sigma \tau\right) v\right) \in N^{E} .
$$

Then $e^{\prime}=g_{\theta}(e)$ satisfies $e_{3}^{\prime}=0, e_{2}^{\prime}=e_{2}+f_{-e_{2}}(0)$ and

$$
e_{1}{ }^{\prime}=e_{1}+2 i F_{0}\left(e_{2}, f_{-e_{2}}(0)\right)+i F_{0}\left(f_{-e_{2}}(0), f_{-e_{2}}(0)\right) \text {. }
$$

As $f_{-e_{2}}(0)=-e_{2}+\mu(0)\left(-e_{2}\right)=-e_{2}, \quad$ now $\quad e_{1}^{\prime}=e_{1}+2 i F_{0}\left(e_{2},-e_{2}\right)$ $+i F_{0}\left(-e_{2},-e_{2}\right)=e_{1}-i F_{0}\left(e_{2}, e_{2}\right), e_{2}^{\prime}=0$ and $e_{3}^{\prime}=0$. Thus

$$
g_{e}(e)=e_{1}-i F_{0}\left(e_{2}, e_{2}\right) \in\left\{v \in \pi^{-1}(0): v_{2}=0\right\} .
$$

Now define $e^{*}=g_{e} \delta g_{e}(e)$. It satisfies $e_{1}^{*}=\delta g_{e}(e)+2 i F_{0}\left(0, f_{-e_{2}}(0)\right)$ $+i F_{0}\left(f_{-e_{2}}(0), f_{-e_{2}}(0)\right)=\delta g_{e}(e)+i F_{0}\left(e_{2}, e_{2}\right)$ and $e_{2}^{*}=f_{-e_{z}}(0)=-e_{2}$. Thus

$$
e^{*}=g_{e} \delta g_{e}(e)=\delta\left(e_{1}-i F_{0}\left(e_{2}, e_{2}\right)\right)+i F_{0}\left(e_{2}, e_{2}\right)-e_{2} .
$$

Now

$$
\begin{aligned}
\left(e^{*}\right)^{*} & =\delta\left(\delta\left(e_{1}-i F_{0}\left(e_{2}, e_{2}\right)\right)+i F_{0}\left(e_{2}, e_{2}\right)-i F_{0}\left(e_{2}, e_{2}\right)\right)+i F_{0}\left(e_{2}, e_{2}\right)+e_{2} \\
& =\delta^{2}\left(e_{1}-i F_{0}\left(e_{2}, e_{2}\right)\right)+i F_{0}\left(e_{2}, e_{2}\right)+e_{2} \\
& =e_{1}-i F_{0}\left(e_{2}, e_{2}\right)+i F_{0}\left(e_{2}, e_{2}\right)+e_{2}=e_{1}+e_{2}=e .
\end{aligned}
$$

Thus $e \rightarrow e^{*}$ is an analytic automorphism of $\pi^{-1}(0)$ with square 1 . If $e=e^{*}$ then

$$
e_{1}=\delta\left(e_{1}-i F_{0}\left(e_{2}, e_{2}\right)\right)+i F_{0}\left(e_{2}, e_{2}\right) \text { and } e_{2}=-e_{2} .
$$


Thus $e_{2}=0$ and $e_{1}=\delta\left(e_{1}\right)$. The latter says $e_{1}=i \xi^{-1}\left(x_{E, \phi}\right)$. Thus $e=e^{*}$ implies $e=i \xi^{-1}\left(x_{E, \phi}\right)$. Now $e \rightarrow e^{*}$ is an analytic automorphism of order 2 on $\pi^{-1}(0)$ with $i \xi^{-1}\left(x_{E, \phi}\right)$ as unique fixed point. As $\pi^{-1}(0)$ is a homogeneous complex manifold holomorphically equivalent to a bounded domain, it follows that $\pi^{-1}(0)$ is a hermitian symmetric space of noncompact type. The tube part of that symmetric space is $\left\{v \in \pi^{-1}(0): v_{2}=0\right\}$, tube domain over $\mathbb{E}_{E}$, holomorphically equivalent to $G_{E, 0}^{E}\left(x_{0}\right)$; in particular $\pi^{-1}(0)$ has rank $|E|$. Statement (3) is proved.

$G_{\Psi \backslash E, 0}$ has Iwasawa decomposition $K_{\Psi \backslash E} \cdot A_{\Psi \backslash E} \cdot U_{\Psi \backslash E}$ and the solvable group $B=A_{\Psi \backslash E} \cdot U_{\Psi \backslash E}$ is simply transitive on $D_{\Psi \backslash E}$. Now define

$$
\lambda: \pi^{-1}(0) \times D_{\Psi \backslash E} \rightarrow \mathfrak{S}_{E} \text { by } \lambda(t, b(0))=b(t) .
$$

Then $\lambda$ is a real analytic trivialization of $\pi: \Im_{E} \rightarrow D_{\Psi \backslash E}$.

Let $\mathcal{G}^{k}$ denote the simple ideals of $\mathcal{G}, \Delta^{k}$ the root system of $\mathcal{G}^{k}$. If $E$ is a union of sets $\Psi \cap \Delta^{k}$, then $G_{0}\left(x_{0}\right)=G_{\Psi \backslash E, 0}\left(x_{0}\right) \times G_{E, 0}\left(x_{0}\right)$ and $\pi$ projects to the first factor. Conversely suppose $\pi$ holomorphically trivial. Let $D_{E}^{\prime}$ denote the bounded symmetric domain in $\mathfrak{N}_{E}^{E-}+Q_{\Psi}^{E-}$ holomorphically equivalent to $\pi^{-1}(0)$. Then there is a holomorphic equivalence of $G_{0}\left(x_{0}\right) \cong \Im_{E}$ with $D_{\Psi \backslash E} \times D_{E}^{\prime}$. As $G_{0}$ is the largest connected group of holomorphic automorphisms of $G_{0}\left(x_{0}\right)$, as $G_{\Psi \backslash E, 0}$ is the largest connected group for $D_{\Psi \backslash E}$, and as the Bergman metric on $D_{\Psi \backslash E} \times D_{E}^{\prime}$ is the direct sum of the Bergman metrics of $D_{\Psi \backslash E}$ and of $D_{E}^{\prime}$, it follows that $G_{0}=G_{\Psi \backslash E, 0} \times G_{E, 0}$ where $G_{E, 0}$ is the largest connected group of holomorphic automorphisms of $D_{E}^{\prime}$. Thus $\mathcal{S}_{\Psi \backslash E}$ is a sum of simple ideals of $\mathcal{G}$, so $\Psi \backslash E$ is a union of sets $\Psi \cap \Delta^{k}$, and we conclude that $E$ is a union of sets $\Psi \cap \Delta^{k}$. Statement (4) is proved, and that completes the proof of Proposition 11A.3. Q.E.D.

\section{REFERENCES}

1. R. Bott and H. Samelson, The cohomology ring of $G / T$, Proc. Nat. Acad. Sci. U.S.A. 41 (1955), 490-493.

2. A. Korányi and J. A. Wolf, Realization of hermitian symmetric spaces as generalized half-planes, Ann. of Math. (2) 81 (1965), 265-288.

3. B. Kostant, On the conjugacy of real Cartan subalgebras, Proc. Nat. Acad. Sci. U.S.A. 41 (1955), 967-970.

4. - Orbits, symplectic structures and representation theory, Proc. U.S. Japan Seminar in Differential Geometry (Kyoto, 1965), Nippon Hyoronsho, Tokyo, 1966.

5. - Lectures on symplectic manifolds and unitary representations, Course given at M.I.T. in Fall, 1965; class notes by N. Iwahori.

6. T. Maebashi, $A$ fixpoint theorem on $\mathrm{Sp}(n) / \mathrm{U}(n)$, Math. J. Okayama Univ. 12 (1966), 91-105.

7. C. C. Moore, Compactifications of symmetric spaces. II: The Cartan domains, Amer. J. Math. 86 (1964), 358-378. 
8. M. Takeuchi, Cell decompositions and Morse inequalities on certain symmetric spaces, J. Fac. Sci. Univ. Tokyo 12 (1965), 81-192.

9. - On orbits in a compact hermitian symmetric space, Amer. J. Math. 90 (1968), 657-680.

10. J. A. Wolf, On the classification of hermitian symmetric spaces, J. Math. Mech. 13 (1964), 489-496.

11. - The geometry and structure of isotropy irreducible homogeneous spaces, Acta Math. 120 (1968), 59-148.

12. - The action of a real semisimple group on a complex flag manifold. II: Unitary representations on partially holomorphic cohomology spaces (in preparation). 13. J. A. Wolf and P. A. Griffiths, Complete maps and differentiable coverings, Michigan Math. J. 10 (1963), 253-255.

14. J. A. Wolf and A. Gray, Homogeneous spaces defined by Lie group automorphisms, J. Differential Geometry 2 (1968), 77-159.

15. J. A. Wolf and A. Korányi, Generalized Cayley transformations of bounded symmetric domains, Amer. J. Math. 87 (1965), 899-939.

University of California, Berkeley, California 94720 